Diss. ETH No. 26358

\title{
TOMOGRAPHY ACROSS SCALES: \\ KNOWLEDGE TRANSFER FROM SEISMOLOGY \\ TO IMAGING BREAST TISSUE WITH ULTRASOUND
}

\author{
A thesis submitted to attain the degree of \\ DoCTOR OF SCIENCES OF ETH ZURICH \\ [DR. SC. ETH ZURICH]
}

presented by

NaiARA Korta Martiartu

MSc. Universitat de Barcelona

born on 12.12 .1986

citizen of Gernika-Lumo, Basque Country

accepted on the recommendation of

Prof. Dr. Andreas Fichtner

Dr. Christian Boehm

Prof. Dr. Hansruedi Maurer

PD Dr. Nicole Ruiter

2020 

"Our feelings are our most genuine paths to knowledge." - Audre Lorde 



\section{Abstract}

Wave propagation is extensively used to understand the internal structure of media that are not accessible to direct observations. Seismology and medical ultrasound imaging are good examples of this. The former uses observations of seismic waves at the Earth's surface to increase our knowledge about its interior. This is crucial, for instance, to improve our understanding about the Earth's dynamics and evolution. Medical ultrasound, on the other hand, uses observations of acoustic waves, emitted and recorded at the surface of human bodies, to visualize internal body structures. This has become an essential screening tool, useful for diagnostic examination.

This thesis presents an interdisciplinary work between seismology and medical ultrasound. In particular, we focus on transferring knowledge from seismic tomography to Ultrasound Computer Tomography (USCT), an emerging technology that holds great potential for early-stage breast cancer diagnosis. Here, the human breast is surrounded by transducers that collect transmitted and reflected ultrasound signals. This information is then used to obtain 3D quantitative images of acoustic tissue properties, which enable non-invasive tissue characterization and improve the specificity of standard imaging modalities. Current challenges in USCT mostly consist in providing a diagnostic tool with high accuracy (comparable to magnetic resonance imaging) and affordable computational and acquisition cost for clinical practice, the target being a maximum time of 15 minutes per patient. Despite the vastly different scale, seismic and medical ultrasound tomography share fundamental similarities that allow us to address these challenges from the stand point of the seismologist.

We first introduce finite-frequency traveltime tomography to medical ultrasound. In addition to being computationally tractable for 3D imaging at high frequencies, the method has two main advantages: (1) It correctly accounts for the frequency dependence and volumetric sensitivity of traveltime measurements, which are related to off-ray-path scattering and diffraction. (2) It naturally enables out-of-plane imaging and the construction of 3D images from 2D slice-by-slice acquisition systems. Our method rests on the availability of calibration data measured in water, used to linearize the forward problem and to provide analytical expressions of cross-correlation traveltime sensitivity. We present a memoryefficient implementation suitable for arbitrarily large-scale domains, and we discuss its extension to amplitude tomography.

To adapt existing acquisition systems to new imaging techniques, we then introduce optimal experimental design methods. These provide a systematic and quantitative framework 
to (1) evaluate the quality of different designs in terms of uncertainties in the estimated tissue parameters and (2) optimize the configuration with respect to predefined design parameters, for example the position of transducers on the scanning device. Our first application presents a cost-effective $3 \mathrm{D}$ configuration of transducers optimized for transmission tomography. This is useful to analyze appropriate quality measures for USCT experiments and explore computationally tractable optimization approaches. The multi-modality capability of USCT, however, requires careful designs that simultaneously provide accurate images for both transmission (e.g., velocity) and reflection (reflectivity) information. We therefore extend the formulation to jointly optimize the experiment for transmission and reflection data. Here we focus on image reconstruction methods with linear(ized) observable-parameter relationship, for which quality measures are analytically given and independent of breast properties. This is crucial for optimizing USCT devices prior to any data acquisition.

Methods investigated within this thesis are validated using experimental data. These contributions represent innovative solutions for USCT and ultimately serve to foster the knowledge and technology transfer between seismology and medical imaging, which may benefit imaging methods on all scales. 


\section{Zusammenfassung}

Wellenausbreitung wird in vielen Bereichen eingesetzt, um die innere Struktur von Materialien zu verstehen, die direkten Beobachtungen nicht zugänglich sind. Zwei gute Beispiele hierfür sind Seismologie und bildgebende Verfahren mit Ultraschall. Erstere Disziplin bedient sich der Beobachtung seismischer Wellen an der Erdoberfläche, um unsere Kenntnise des Erdinnern zu erweitern. Dies ist unabdingbar, um unser Verständnis von dessen Dynamik und Evolution zu verbessern. Die bildgebenden Verfahren mit Ultraschall verwenden dagegen Beobachtungen akustischer Wellen, die an der Oberfläche des menschlichen Körpers angeregt und aufgenommen werden, um Strukturen im Körperinnern zu visualisieren. Sie haben sich zu einem wichtigen Instrument in der Früherkennung entwickelt, welches der diagnostischen Untersuchung dient.

Die vorliegende Arbeit stellt ein interdisziplinäres Projekt zwischen Seismologie und medizinischem Ultraschall vor. Ihr Fokus liegt insbesondere auf dem Methodentransfer von der Seismologie zur Ultraschall-Computertomographie (Ultrasound Computer Tomography, USCT), einer sich rasch entwickelnden Technologie mit weitreichendem Potential für die Brustkrebs-Früherkennung. In USCT wird die menschliche Brust von Ultraschallsensoren umgeben, welche sowohl transmittierte als auch reflektierte Ultraschallsignale aufnehmen. Diese Informationen werden genutzt, um quantitative Abbildungen der akustischen Eigenschaften des Gewebes in 3D zu erstellen, was die nichtinvasive Charakterisierung des Gewebes ermöglicht und die Spezifizität gegenüber Standardverfahren verbessert. Die derzeitigen Herausforderungen in USCT bestehen vor allem darin, ein diagnostisches Werkzeug mit hoher Genauigkeit (vergleichbar der Magnetresonanztomographie) bereitzustellen, dessen Mess- und Berechnungsdauer für die klinische Praxis akzeptabel sind, wobei das Ziel bei 15 Minuten pro Patient*In liegt. Trotz der sehr unterschiedlichen räumlichen Skalen bestehen fundamentale Ähnlichkeiten zwischen seismischer und medizinischer Tomographie, sodass sich die Möglichkeit bietet, Herausforderungen in USCT vom seismologischen Standpunkt aus zu betrachten.

Wir übertragen zunächst die Laufzeittomographie mit finiten Frequenzen in die Ultraschallbildgebung. Ihre schnelle Berechenbarkeit erlauben das Erstellen dreidimensionaler Abbildungen, und sie hat darüber hinaus zwei wesentliche Vorteile: (1) Sie gibt die Frequenzabhängigkeit und die volumetrische Sensitivität der Laufzeiten korrekt wieder, welche durch Streuung und Beugung abseits des Strahlwegs gegeben sind. (2) Sie ermöglicht die Bildgebung ausserhalb der Ebene (out-of-plane imaging) und das Erstellen von 3D Abbildungen 
mit 2D Messgeräten, die sequentielle Messungen einzelner Querschnitte durchführen. Unsere Methode beruht auf der Verfügbarkeit von in Wasser gemessenen Kalibrationsdaten, die genutzt werden, um das Vorwärtsproblem zu linearisieren und analytische Lösungen für die Sensitivität der Laufzeiten anzugeben. Wir präsentieren eine Implementierung mit effizienter Nutzung des Arbeitsspeichers, die für beliebig grosse Modelle eingesetzt werden kann, und diskutieren ihre Erweiterung für Tomographie anhand von Amplitudenmessungen.

Um bestehende Messsysteme an neue bildgebende Verfahren anzupassen, führen wir danach Methoden der optimalen Versuchsplanung (optimal experimental design, OED) ein. Diese bieten einen systematischen und quantitativen Rahmen, um (1) die Qualität verschiedener Messanordnungen in Bezug auf die Unsicherheiten der abgebildeten Gewebeparameter zu bewerten und (2) die Messanordnung unter Berücksichtigung vorgegebener Parameter, wie zum Beispiel der Position der Sensoren auf dem Scanner, zu optimieren. Als erste Anwendung stellen wir eine kosteneffektive, für Transmissionstomographie optimierte 3D Konfiguration von Ultraschallsensoren vor. Dies ist nützlich, um entsprechende Qualitätskriterien für USCT-Experimente zu analysieren und Optimierungsansätze mit adäquaten Rechenanforderungen zu erkunden. Allerdings erfordern die verschiedenen USCTModalitäten sorgfältige Messdesigns, die sowohl für Transmissions- (z.B. Geschwindigkeit) als auch für Reflektionsinformation (Reflektivität) Resultate mit hoher Genauigkeit ergeben. Deshalb erweitern wir die Formulierung des OED-Problems, um die Anordnungen für Transmissions- und Reflektionsexperimente gleichzeitig zu optimieren. Im Fokus stehen bildgebende Verfahren mit linearem oder linearisiertem Zusammenhang zwischen Modell und Parametern, da deren Qualitätsgrössen analytisch beschrieben werden können und sie unabhängig von den Eigenschaften des Brustgewebes sind, was unabdingbar ist, um das Design von USCT-Messgeräten unabhängig von einzelnen Messungen zu optimieren.

Die hier untersuchten Methoden werden mit Messdaten von Labor-Experimenten validiert. Die Beiträge dieser Arbeit stellen innovative Lösungen für USCT dar, und fördern den Methoden- und Technologietransfer zwischen Seismologie und Ultraschall-Imaging, von dem bildgebende Verfahren auf allen Skalen profitieren können. 


\section{Contents}

$\begin{array}{lc}\text { Abstract } & \text { i }\end{array}$

$\begin{array}{lr}\text { Zusammenfassung } & \text { i i i }\end{array}$

$\begin{array}{ll}\text { List of Figures } & \mathrm{x}\end{array}$

$\begin{array}{lr}\text { 1. Breast cancer diagnosis } & 7\end{array}$

2. Ultrasound Computer Tomography $\quad 9$

2.1. Brief historical background . . . . . . . . . . . . . . . . . 10

2.2. Current research ....................... 11

2.2.1. Reconstruction methods . . . . . . . . . . . . . . . . 12

2.2.2. Acquisition systems . . . . . . . . . . . . . . . . 13

2.3. New challenges .......................... 15

3. Seismic tomography $r$

3.1. Brief historical perspective . . . . . . . . . . . . . . . . . . . . 18

3.2. Context: Similarities and differences . . . . . . . . . . . . . . . . 19

3.3. Lessons from seismology . . . . . . . . . . . . . . . . . . . . . 21

3.3.1. Optimal Experimental Design . . . . . . . . . . . . . . 22

3.3.2. Finite-frequency tomography . . . . . . . . . . . . . . 23

3.4. Thesis: Objective and Outline . . . . . . . . . . . . . . . . 24

4. Image reconstruction $\quad 27$

4.1. Forward theory . . . . . . . . . . . . . . . . . . . . 27

4.1.1. Ray-based methods: Infinite-frequency . . . . . . . . . . . . . . . . . 29

4.1.2. Wave-based methods: Finite-frequency . . . . . . . . . . . . . . 32

4.1.3. Sensitivities in the Jacobian operator . . . . . . . . . . . . . 35

4.2. Inverse theory . . . . . . . . . . . . . . . . . . . . . . . . 38

4.2.1. Properties of the forward operator . . . . . . . . . . . . 38

4.2.2. Bayesian inverse problem . . . . . . . . . . . . . . . 42

4.2.3. Deterministic inversion . . . . . . . . . . . . . . . 43

4.2.4. Regularization approaches .................... 44 
4.2.5. Uncertainty and resolution analysis . . . . . . . . . . . . . . 49

4.3. Comments on waveform tomography . . . . . . . . . . . . . 52

5. Finite-frequency tomography $\mathbf{5 5}$

5.1. Finite-frequency traveltime tomography . . . . . . . . . . . . . 56

5.1.1. Traveltime Fréchet derivatives . . . . . . . . . . . . . . . 56

5.1.2. Linearization . . . . . . . . . . . . . . 58

5.1.3. 2D approximation . . . . . . . . . . . . . 59

5.1.4. New parameterization of the forward operator . . . . . . . . . 60

5.2. 2 D lab data application . . . . . . . . . . . . . . . . 63

5.3. 3D reconstruction with $2 \mathrm{D}$ lab dataset $\ldots \ldots \ldots \ldots . \ldots 71$

5.4. Simulated full 3D experiment: slice-by-slice acquisition . . . . . . . . . . 73

5.5. Discussion and conclusion . . . . . . . . . . . . . . . 76

5.6. Outlook: amplitude measurements . . . . . . . . . . . . . . . . . . . . . . . 77

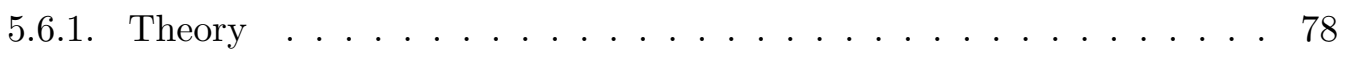

5.6.2. Numerical results . . . . . . . . . . . . . . . . . . . . . . 81

5.6.3. Lab data application . . . . . . . . . . . . . . . . . . . . . . . . . . 83

5.6.4. Conclusion ..................... . . 83

6. Designing USCT experiments: Part I $\quad 85$

6.1. Optimal experimental design . . . . . . . . . . . . . 86

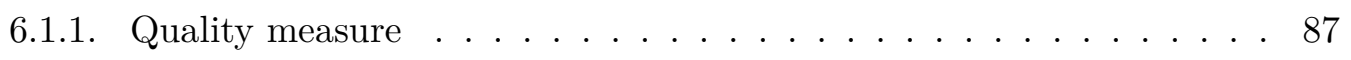

6.1.2. Optimization strategies . . . . . . . . . . . . . . 90

6.2. Numerical results: 3D hemispherical setup . . . . . . . . . . . . . . . . . . . . . . . . . . . . .

6.2.1. Building a suitable initial configuration . . . . . . . . . . . 93

6.2.2. Full 3D optimization . . . . . . . . . . . . . . . . . . . . 94

6.2.3. Validation of SOED approach . . . . . . . . . . . . . . . 99

6.3. Discussion . . . . . . . . . . . . . . . . . . . . . . . . . 101

6.4. Conclusions . . . . . . . . . . . . . . . . . . . . 103

6.5. Outlook: practical extension to waveform tomography . . . . . . . . . . 104

6.5.1. Numerical example . . . . . . . . . . . . . . . . . . . . . . 105

7. Designing USCT experiments: Part II 109

7.1. Joint optimization of the experimental design . . . . . . . . . . . . . . . . . . . . . . . . . . . . . .

7.1.1. Joint posterior covariance . . . . . . . . . . . . . . . 110

7.1.2. Quality measure . . . . . . . . . . . . . . . 110

7.1.3. Non-Gaussian priors . . . . . . . . . . . . . . . . . . . 111

7.1.4. Application to imaging techniques . . . . . . . . . . . . . 112

7.2. Toy example . . . . . . . . . . . . . . . . . . . . . . . . 112

7.2.1. Sensitivity of the acquisition system . . . . . . . . . . 113 
7.2.2. Individual optimizations . . . . . . . . . . . . . . . . . . . 113

7.2.3. Joint optimization . . . . . . . . . . . . . . . . . . . . 114

7.2.4. Comment on the parameterization . . . . . . . . . . 115

7.3. Real data application: emitter selection . . . . . . . . . . . 117

7.4. Discussion and Conclusion . . . . . . . . . . . . . . . . 120

7.5. Outlook: Sensitivity of the quality measure . . . . . . . . . . . . . 122

$\begin{array}{lr}\text { 8. Discussion and final remarks } & \mathbf{1 2 7}\end{array}$

8.1. From seismology to USCT . . . . . . . . . . . . . . . . 127

8.2. Specific contributions . . . . . . . . . . . . . . . . . 128

8.3. Cross-fertilization: back to seismology? . . . . . . . . . . . . . . . . . . 129

$\begin{array}{ll}\text { A. Born approximation } & 131\end{array}$

B. Low-rank approximation $\quad 133$

B.1. Posterior covariance matrix . . . . . . . . . . . . . . . . 133

B.2. Resolution matrix . . . . . . . . . . . . . . . . . . . 134

$\begin{array}{ll}\text { References } & 135\end{array}$

$\begin{array}{ll}\text { Acknowledgments } & 157\end{array}$

$\begin{array}{lr}\text { Curriculum Vitae } & 159\end{array}$

$\begin{array}{lr}\text { Permissions } & 161\end{array}$ 



\section{List of Figures}

Figure 2.1. Wave propagation in USCT. . . . . . . . . . . . . . . . . . 9

Figure 2.2. Empirical acoustic tissue properties. . . . . . . . . . . . . . 10

Figure 2.3. Historical USCT reconstruction. . . . . . . . . . . . . . . . . . . 11

Figure 2.4. QT Scanner. . . . . . . . . . . . . . . . . . . . 14

Figure 2.5. KIT 3D USCT system. . . . . . . . . . . . . . . . . . . 15

Figure 3.1. Collaborative seismic earth model. . . . . . . . . . . . . . . 18

Figure 3.2. Comparison between brest tissue and Earth's mantle. . . . . . . . . 21

Figure 4.1. Measurement sensitivity to tissue parameters in image reconstruction. 37

Figure 4.2. Illustrative experiment to introduce inverse theory. . . . . . . . . . . 39

Figure 4.3. Singular value spectrum of the forward operator. . . . . . . . . . . 40

Figure 4.4. Eigenvectors of the forward operator. . . . . . . . . . . . . . . . . 41

Figure 4.5. Comparison of different regularization approaches. . . . . . . . . . . 46

Figure 4.6. Regularized eigenvalue spectrum of the Hessian. . . . . . . . . . . 47

Figure 4.7. Posterior covariance and resolution matrix. . . . . . . . . . . . . 50

Figure 5.1. Traveltime sensitivity kernel. . . . . . . . . . . . . . . . . 60

Figure 5.2. Pattern in sensitivity kernels. . . . . . . . . . . . . . . . . 62

Figure 5.3. Lab experiment. . . . . . . . . . . . . . . . . . 63

Figure 5.4. Reconstruction and sensitivity coverage. . . . . . . . . . . 65

Figure 5.5. Singular value spectrum, posterior covariance and resolution matrix. 67

Figure 5.6. Point-spread functions. . . . . . . . . . . . . . . . . . . . 69

Figure 5.7. Solution without nullspace. . . . . . . . . . . . . . 70

Figure 5.8. 3D velocity reconstruction and resolution analysis. . . . . . . . . . 72

Figure 5.9. Full 3D velocity reconstruction. . . . . . . . . . . . . . . . . 74

Figure 5.10. Resolution analysis for full 3D reconstruction. . . . . . . . . . . . 75

Figure 5.11. Amplitude and traveltime sensitivity kernels. . . . . . . . . . . . 80

Figure 5.12. Reflectivity and velocity reconstructions using amplitudes. . . . . . . 82

Figure 5.13. True velocity model for synthetic experiment. . . . . . . . . . . . . 83

Figure 5.14. Lab data velocity reconstruction using amplitudes. . . . . . . . . . . 84

Figure 6.1. Reference 3D experimental setup . . . . . . . . . . . . . . 86 
Figure 6.2. Quality measure in toy example. . . . . . . . . . . . . . . . . 89

Figure 6.3. Benefit-cost curve. . . . . . . . . . . . . . . . . . . . 92

Figure 6.4. Numerical phantom and reference setup. . . . . . . . . . . . . . . 94

Figure 6.5. Optimized positions of transducers in 2D examples. . . . . . . . . 95

Figure 6.6. 3D optimized experimental design. . . . . . . . . . . . . . . . . 97

Figure 6.7. Eigenvalue spectra for optimized and reference setups. . . . . . . . . 98

Figure 6.8. Velocity reconstructions from optimized designs. . . . . . . . . . . . 98

Figure 6.9. Optimized experimental designs from GOED and SOED. . . . . . . 100

Figure 6.10. Eigenvalue spectra for GOED and SOED, and benefit-cost curve. . . 100

Figure 6.11. Discussing the symmetry of the optimized configuration. . . . . . . . 102

Figure 6.12. SOED for FWI: 2D numerical example. . . . . . . . . . . . . . 106

Figure 6.13. SOED for FWI: 2D numerical example II. . . . . . . . . . . . . . . . 107

Figure 6.14. SOED for FWI: eigenvalue spectra for 2D numerical examples. . . . 107

Figure 7.1. Sensitivity coverage for individual imaging techniques. . . . . . . . 114

Figure 7.2. Toy exmaple: individual optimizations. . . . . . . . . . . . . . . . 115

Figure 7.3. Toy example: Joint transmission-reflection D-SOED. . . . . . . . . . 116

Figure 7.4. Effect of the parameterization on the benefit-cost curves. . . . . . . 117

Figure 7.5. Real data application: Joint reflection-transmission D-SOED and reconstructions. . . . . . . . . . . . . . . . . . . 118

Figure 7.6. Reconstructions at different stages of the benefit-cost curve. . . . . . 119

Figure 7.7. Reflection information reconstruction for real data application. . . . 120

Figure 7.8. Sensitivity of the quality measure. . . . . . . . . . . . . . . . 124 


\section{List of Tables}

3.1. Scale characteristics in exploration seismic and USCT. . . . . . . . . . . 20

6.1. Comparison of quality measures in toy example. . . . . . . . . . . 88

6.2. Estimates of quality measure for FWI using optimized designs. . . . . . . 103 



\section{Preface}

"Science, it would seem, is not sexless; she is a man, a father and infected too."

- Virginia Woolf, Three Guineas (1938)

Contributing to societal aspects that are directly relevant to historically marginalized groups was my main motivation to start this thesis. Despite the rapid rise in gender awareness experienced in the last decades, it is widely accepted that gender-hierarchybased structures are still prevalent in our societies (e.g., Butler, 2002; Davis, 2011). In such context, and as a female* researcher, the topic of breast cancer diagnosis becomes fascinating at different levels. This is not only an opportunity to contribute to women*'s health issues, which have been historically marginalized or neglected from medical research (e.g., Schiebinger, 2000), but also to place myself simultaneously as both the subject and the potential object of these studies. Bringing such ambivalent perspectives can enrich the non-epistemic or contextual values that are inherent to scientific practice and ultimately affect the scientific objectivity (e.g., Tuana, 1989; Hubbard, 1990; Haraway, 1991; Harding, 1992; Keller, 1996). In particular, new and diverse perspectives may be crucial to eradicate sexualized representations of breast cancer, and female* breast in general, that still exist in scientific environments and reinforce gender-normative stereotypes (personally experienced during this thesis).

Various philosophers of science, including feminist and poststructuralist scholars, have for a long time challenged the traditional scientific view on the subject-object division (e.g., Feyerabend, 1962; Latour, 1999; Daston and Galison, 2007). Scientific objectivity is (still, I would argue) conceived as a view from nowhere, abstracting from the perspective of the individual scientist and involving, therefore, a radical objectivity-subjectivity division. In other words, it is conceived as value-neutral and universal. Science, however, being a social activity in itself, cannot be understood out of any socio-historical, political and cultural context that creates it. Such contexts shape the scientific discoveries, narratives and inquires (e.g., Martin, 1991; Keller, 1991; Potter, 2001), and they cannot be neglected for a comprehensive understanding of the scientific knowledge. Particular to the topic of this thesis is the critical role of breast cancer survivors, activists, and movements, who

*I choose to use traditional gender binary categories for strategical reasons and clarity. However, the asterisk is a reminder of the plurality and complexity of identities that nowadays are emerging to challenge the simplistic and dominant understanding of the social reality. 
challenged the medical research establishment and shaped the scientific research on breast cancer screening, treatment and prevention. Thus, I could not start this thesis without first acknowledging their contributions. Guided by the available literature, I will briefly introduce breast cancer movements in the United States.

Nowadays, the pink ribbon or fund-raising and awareness races became part of our social imaginary linked to breast cancer. Before developing into a pink business of unexpected dimensions, however, breast cancer was considered an unspeakable condition until the 20th century (Osuch et al., 2012). Women* with breast cancer suffered from the structural stigma, isolation, and invisibility, which led them to experience the disease privately and silently. During the 1970s and 1980s the standard medical treatment consisted in radical mastectomies (Osuch et al., 2012), i.e., the removal of the whole breast, lymph nodes of the axilla and muscles of the chest wall, making the illness experience highly dramatic. After decades of silence, the situation drastically changed by the 1990s; breast cancer came out of the closet with empowered women* claiming visibility in the public space (Klawiter, 2008). The experience of being diagnosed with breast cancer transformed into a political identity, being collectively shared and publicly declared. This was definitely not accidental; it was the result of committed activism, largely influenced by (1) the women*'s health movements driven by the second-wave feminism in the 1970s, and (2) the exemplary HIV/AIDS activism of gay, lesbian, and trans movements in the 1980s (Klawiter, 2008). To understand the impact of this transformation on the illness experience of individual women*, I refer the reader to Klawiter (2004), who analyzes the narrative of Clara Larson, a woman from San Francisco Bay Area diagnosed twice with breast cancer in 1979 and 1997.

The synergies between HIV/AIDS and feminist breast cancer activists is specially remarkable in California. For instance, the Women's Cancer Resource Center (WCRC) was co-founded in 1986 by Jackie Winnow, who was the coordinator of the LGTB+ and AIDS Discrimination Unit in San Francisco, and was diagnosed with breast cancer. At that time, San Francisco had the reputation of the breast cancer capital of the world, as a result of rapidly growing cancer registries. WCRC was the first agency of its kind in the country, and it was committed "to empower women with breast cancer to be active and informed about their disease; to provide community for women with cancer and their supporters; to educate the general public about cancer; and to be actively involved in the struggle for a lifeaffirming, cancer-free society" (Klawiter, 2008). WCRC clearly extended the AIDS model of organizing to women* with cancer, and it was immediately followed by the foundation of similar organizations in the next years. Together they created a culture of breast cancer activism that "pushed science to develop effective, innovative, nontoxic forms of screening, detection and treatment" (Klawiter, 2008, p.197). As a consequence, researchers discovered, for instance, that the gene $e r b B 2$ is overexpressed in approximately $30 \%$ of human breast cancers, and this made possible the subsequent development of erbB2-targeting antibody 
for treatment (Slamon et al., 1989; Lerner, 2002).

These are just very specific examples useful to remind us the societal aspects of our research, beyond the obvious ones. Here, our objects of study are not passive bodies, but subjects with political agency that claim their (our) direct participation in more democratic scientific and medical research processes. This is also my personal attempt to claim for a more inclusive science that recognizes the value of diversifying the perspectives of social subjects participating in it.

Zurich, Switzerland

Naiara Korta Martiartu

September 6, 2019 



\section{Breast cancer diagnosis}

Breast cancer is becoming an increasingly common disease that principally, but not exclusively, affects women*'s bodies. In Europe, for instance, it has become the most common cancer in 2018, with 523000 new cases (13.4\% of all cancer cases reported) (Ferlay et al., 2018). For women*, in particular, it is the leading overall cause of death from cancer, although the mortality rate has shown a favourable trend since the mid 1980's. For example, European rates declined by $15 \%$ between 2002 and 2012, and a further $10 \%$ decline is expected by 2020 (Carioli et al., 2017). Several factors are attributed to the falls in mortality that essentially include improvements in the diagnosis, treatment and management of breast cancer over the last three decades (Carioli et al., 2017). Of particular interest to this thesis are the advancements in diagnosis that contribute to early-stage detection.

Periodical examination of the breast is essential for early detection, and systematic screening programs have been introduced in many countries. Mammography is currently the standard screening technology, although its benefits and harms are the source of controversies and debates. In Switzerland, for instance, while screening is recommended every 2 years for women* aged between 50 and 69 years (Perry et al., 2008), the Swiss Medical Board, an independent health technology assessment consortium, has recently suggested to abandon mammography screening programs (Biller-Andorno and Jüni, 2014). The concerns are mostly related to the high level of overdiagnosis and overtreatment induced by mammography (Barratt, 2015), i.e., the detection and treatment of tumors that are not life threatening, exposing patients to adverse physical and psychological effects that are avoidable. Other limitations of mammography include (1) radiation exposure, (2) low sensitivity in patients with dense breast tissue (false-negative results), and (3) risk for false positives in early detection and consequent unnecessary biopsies. The controversial statement of the Swiss Medical Board was received with criticism by Swiss institutions and became mostly anecdotal (Chiolero and Rodondi, 2014), but, in any case, it highlights the need for alternative imaging technologies with favourable benefit-harm balance (Autier and Boniol, 2018).

Ideally, an imaging technique used for routine breast screenings must provide high sensitivity and specificity to breast cancer, while being cost effective and non invasive. Currently, there are various techniques that are used as complementary to mammography:

1. Digital breast tomosynthesis (DBT): DBT uses X-rays to provide volumetric images 


\section{Breast cancer diagnosis}

of breast tissue from a set of $2 \mathrm{D}$ projections. By reducing tissue overlapping, it has the potential to improve the sensitivity, reduce false positives and facilitate accurate differentiation of lesion types, at the cost of an increased exposure to ionizing radiation. This is probably its major limitation.

2. Magnetic resonance imaging (MRI): MRI shows higher sensitivity than mammography (Sardanelli et al., 2004), and it has become the principal adjunct imaging method for high-risk populations (Saslow et al., 2007). It provides volumetric reconstructions of breast tissue from cross-section images, being moreover radiation-free. However, MRI shows lower specificity values, leading to high false positive rate, requires contrast injection and the cost of the examination is very high ${ }^{1}$. These exclude the broad use of MRI for general population.

3. Hand-held ultrasound: Unlike MRI and DBT, hand-held ultrasound is radiation-free and relatively inexpensive. It is the standard adjunct modality for differentiating cysts from tumors, and, in combination with mammography, the cancer detection may be increased by $50 \%$ for high-risk patients with dense breast tissue (Berg et al., 2008). However, it may result in false-positive findings, and its performance will highly depend on the skills of the operator, hindering reproducibility.

Between the mentioned techniques, ultrasound shows the most attractive and flexible characteristics (non invasive and cost effective) for routine breast screening. To further improve the performance of this modality, Automatic Breast Ultrasound Systems (ABUS) have been developed (e.g., Kaplan, 2014). This technology is an extension of hand-held ultrasound that increases the reproducibility by automatizing the acquisition procedure. It uses a larger transducer aperture that enables whole breast imaging, while reducing the examination time. Although ABUS detects cancers that mammography misses, it does not show higher sensitivity for all cancer types, and thus, it can only be used as adjunct modality to mammography (Brem et al., 2015). Both ABUS and hand-held ultrasound rely on reflected ultrasonic signals to provide B-mode images of tissue reflectivity. These are qualitative images useful to differentiate benign and malign lesions through anatomical information of breast structure, i.e., mass margins and shape among others. ABUS, therefore, suffers from the same low specificity issues as conventional ultrasound, and systems that acquire different information are essential to further improve this aspect. In this light, Ultrasound Computer Tomography (USCT) may create a paradigm shift in the performance of ultrasound systems. In the next chapter, we will introduce this technology, describe its historical development and current state of the art. This will be useful to understand the new challenges that arise in USCT, and we will conclude by formulating several questions that are addressed during this thesis.

\footnotetext{
${ }^{1}$ The cost for MRI screening can vary in the range of $476.51-1432.84$ USD whereas for mammography the range is $28.37-73.65$ USD (Moore et al., 2009).
} 


\section{Ultrasound Computer Tomography}

Ultrasound Computer Tomography (USCT) is an emerging technology for breast cancer diagnosis. In this technique, ultrasonic waves are propagated through the tissue and recorded by a set of transducers that are surrounding the breast [see Figure 2.1]. In contrast to conventional hand-held ultrasound, USCT systems collect both reflection and transmission information, which are then used to obtain quantitative images of acoustic tissue properties. These typically include sound speed, reflectivity and attenuation. Empirical studies have demonstrated the diagnostic value of this information, enabling non-invasive tissue characterization. In particular, they can help to differentiate tumors from benign lesions (Stavros et al., 1995; Greenleaf et al., 1977), thereby improving the specificity of standard imaging modalities. Although this may be the most important characteristic of USCT, it also has other advantages: it is ionizing radiation and breast compression free, relatively inexpensive and operator independent. The latter is essential to enable reproducible images of the whole breast.

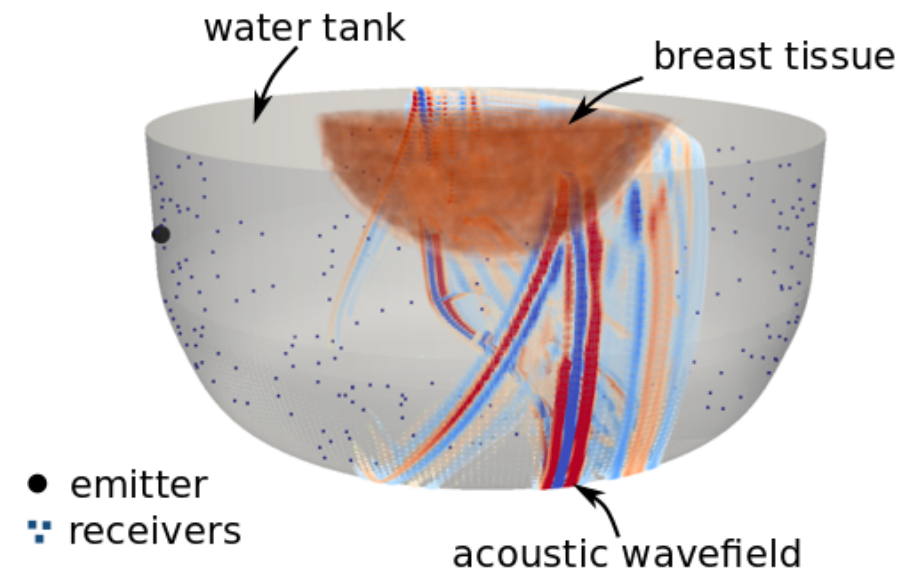

Figure 2.1.: Simulation of ultrasonic wave propagation (Afanasiev et al., 2019) through a realistic numerical breast phantom (Lou et al., 2017) in 3D USCT scanning systems. 


\subsection{Brief historical background}

The potential of USCT has been known since the 1970s (Green et al., 1974; Heyser and Croissette, 1974; Schomberg, 1978). Initial attempts were possibly inspired by the pioneering work done by Hounsfield (1973), who developed the X-ray computerized tomography (CT). The first description of ultrasound transmission tomography was made by Greenleaf and Johnson (1975), and similarly to X-ray tomography, they were relying on straight-ray assumptions to image two-dimensional (2D) projections of sound speed and attenuation distributions of breast tissue. Their scanning system was able to acquire $2 \mathrm{D}$ data from an emitter-receiver geometry that covered $360^{\circ}$ using rotations. The system would require the patient lying prone with the breast submerged in a water tank, while the device was scanning the breast in coronal slices. In a subsequent study from the same group, the device was used to image the breast of approximately 150 patients, from which 30 had biopsyconfirmed cancer (Greenleaf and Bahn, 1981). This study empirically demonstrated the possibility for differential diagnosis of pathologies on the basis of acoustic tissue properties: different types of tissue, including malign and benign lesions, could be characterized and classified using quantitative sound speed and attenuation measurements. Their finding, shown in Figure 2.2, was key to understand the relevance of USCT systems in breast cancer diagnosis.

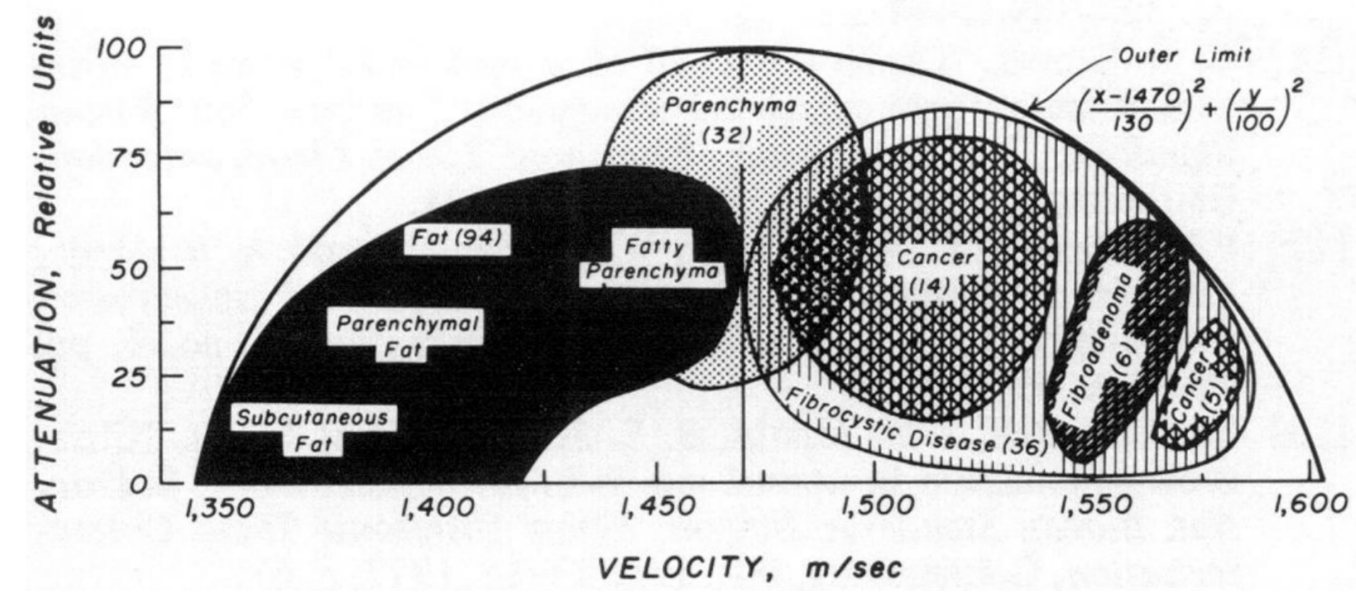

Figure 2.2.: Empirical sound speed and attenuation measurements for different types of breast tissue. Modified after Greenleaf and Bahn (1981) @[1981] IEEE.

During the same period, other groups also reported successful applications of ultrasound transmission tomography to in vivo data (Glover, 1977; Glover and Sharp, 1977). The goal of these studies was mainly focused on providing an alternative to traditional pulse-echo ultrasound techniques, and therefore, reflection or B-mode imaging was not integrated into their systems. Carson et al. (1981), however, showed the potential of combining 
pulse-echo images with transmission tomography. Using similar $2 \mathrm{D}$ systems, they were able to provide simultaneous images of reflectivity, sound speed and attenuation for the same coronal planes. An example of their results is shown in Figure 2.3, which suggested complementary roles of different acoustic tissue parameters in breast cancer detection. A decade later, this was confirmed by Stavros et al. (1995), who demonstrated the importance of reflection images in the differentiation of benign from malignant solid nodules. Using images acquired by conventional sonography techniques, their analysis was based on the observation of mass margins, shape and echo properties of the heterogeneities, reporting a sensitivity for cancer of $98.4 \%$. These were promising results for USCT, which could further increase the diagnostic accuracy of medical ultrasound by reducing the dependence on the operator skills.
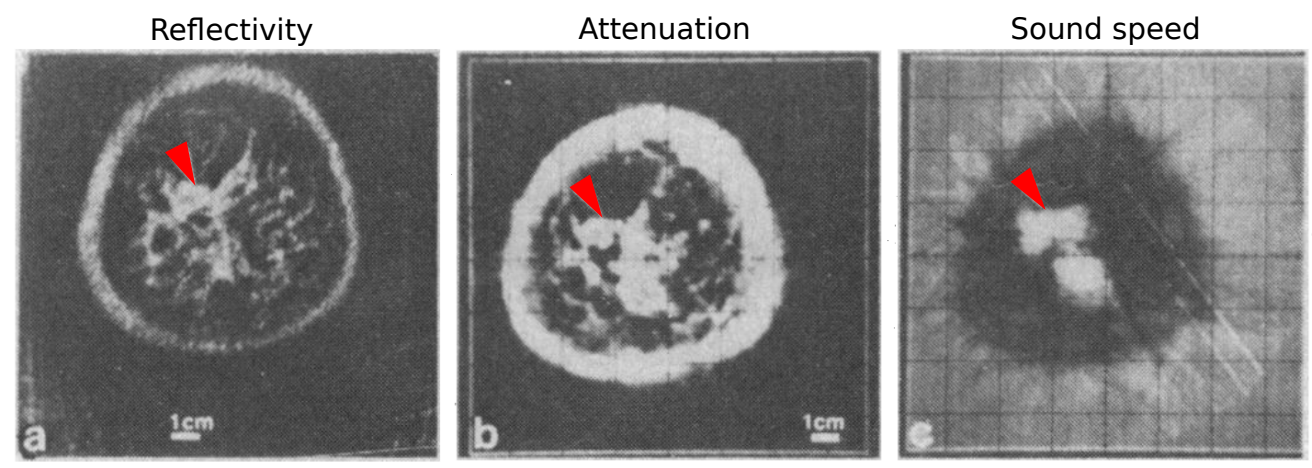

Figure 2.3.: Coronal images of reflectivity, attenuation and sound speed obtained simultaneously from the breast of a 55-year-old woman. Red arrows indicate the position of ductal carcinoma observed in all images. Reprinted from Carson et al. (1981), with permission from AAAS.

Despite the promising opportunity for USCT, in the following decades little progress was made in further developing the technology. The early prototypes were very slow and impractical, limited by available computing power and hardware technologies (André et al., 2013). At that time, USCT was considered premature (Carson and Fenster, 2009), and only in the last decade, due to new technologies and continuous scientific progress, it has emerged as an active field of research (Gemmeke and Ruiter, 2007; Pratt et al., 2007; Roy et al., 2010; Wiskin et al., 2012; Ozmen et al., 2015; Sandhu et al., 2015; Pérez-Liva et al., 2017a). Currently, several USCT systems are already approaching clinical utility (Duric et al., 2014; Wiskin et al., 2013; Gemmeke et al., 2017).

\subsection{Current research}

The main challenge addressed by the current studies is to provide a diagnostic tool with high accuracy and affordable acquisition and computational cost for clinical practice (e.g., Wang et al., 2015; Matthews et al., 2017; Ihrig and Schmitz, 2018; Taskin et al., 2018). 


\section{Ultrasound Computer Tomography}

The expected accuracy and time to solution is often established in comparison to MRI, giving a target for the latter of approximately 15 minutes per patient. This trade-off can be controlled by the choice of the reconstruction method as well as the setup of the scanning device, and therefore, current studies are mainly focused on these two directions. Although this may be a simplification of the literature, it facilitates the understanding of relevant aspects that we want to address in the context of this thesis.

\subsubsection{Reconstruction methods}

As mentioned before, typical USCT devices are designed to simultaneously image multiple acoustic parameters, which can be divided in either reflection or transmission properties. For reflection imaging, B-mode, synthetic aperture focusing techniques (SAFT) or delayand-sum (DAS) beamforming are relatively well established (Jensen et al., 2006; Duric et al., 2007; Schmidt et al., 2011; Gemmeke et al., 2017). They are based on ray theory and therefore use simplified wave propagation models that neglect off-ray-path scattering and diffraction. These methods are robust and fast (easily parallelizable), and for high-frequency data $(>1 \mathrm{MHz})$, they can provide high-quality images of the reflectivity distribution, with submillimeter resolution (Stotzka et al., 2005). Recently, attempts to incorporate more accurate physical models were reported (Roy et al., 2016; Pratt, 2018), and the reversetime migration (RTM) technique has been introduced to USCT. Although RTM is more computationally intensive than the B-mode technique, it has the potential to provide accurate images for much lower frequencies (Roy et al., 2016). As we discuss latter, this may be beneficial for scanning systems adapted to waveform tomography.

Sound speed, or velocity, is the most studied transmission property due to its strong correlation with tissue density (Mast, 2000; Sak et al., 2017), a risk factor for breast cancer (Harvey and Bovbjerg, 2004). Typical USCT systems use ray-based tomographic algorithms to reconstruct this information from the first-arrival times ( $L i$ et al., 2010; Pérez-Liva et al., 2015; Gemmeke et al., 2017). These methods are robust and computationally efficient, making them very attractive for clinical practice. However, being based on the infinite-frequency assumptions, they neglect two essential aspects of the traveltime measurements: (1) In practice, these are estimated by cross-correlating the observed signals with calibration data, and therefore, they depend on the finite-frequency effects contained in the cross-correlated pulses. (2) Due to scattering and diffraction effects, the traveltimes of the finite-frequency ultrasonic waves are sensitive to tissue structure off the ray path. Consequently, approximations made in ray theory are only valid for media with heterogeneities larger than the wavelength and the first Fresnel volume (Williamson, 1991). Similar algorithms are used for attenuation reconstruction, using in this case amplitude information of the first-arrival pulses ( Li et al., 2008).

To improve the spatial resolution of transmission reconstructions, significant efforts have 
been made to introduce imaging techniques that rely on more accurate descriptions of wave propagation (Lavarello and Hesford, 2013; André et al., 2013). Lavarello and Hesford (2013) published an exhaustive review on different forward and inverse problems applied in USCT. Among all these techniques, waveform tomography has shown the most promising results in terms of spatial resolution and contrast (Pratt et al., 2007; Ozmen et al., 2015; Goncharsky et al., 2016; Pérez-Liva et al., 2017b; Boehm et al., 2018; Calderón Agudo et al., 2018). The method accounts for all wave phenomena by numerically solving the wave equation, and with sufficient data coverage and quality, it can provide images with subwavelength spatial resolution. Applications to in vivo data using slice-by-slice scanning systems have shown very promising results (Pratt et al., 2007; Sandhu et al., 2015). However, new challenges arise: (1) The computational burden increases significantly with respect to ray-based approaches, especially for high frequencies or 3D reconstructions. Here, experiments in which the number of wavelengths propagated from emitters to receivers exceeds 100 are considered as high frequency ones (Pratt, 2018). (2) The optimization problem becomes highly non-linear, being sensitive to cycle-skipping and local minima (Gauthier et al., 1986; Bunks et al., 1995). Adapted acquisition systems that provide cost-effective designs and low-frequency data are therefore essential to alleviate the cost and to ensure meaningful solutions (Calderón Agudo, 2018; Fichtner, 2010). As example of computational cost in waveform tomography, the reader is referred to Calderón Agudo et al. (2018) for quantitative information about the cost for $2 \mathrm{D}$ and $3 \mathrm{D}$ reconstructions using frequencies below 1.5 $\mathrm{MHz}$.

\subsubsection{Acquisition systems}

During the last decade, several transducer setups have been investigated, ranging from circular to semi-ellipsoidal distributions (Johnson et al., 2007; Schwarzenberg et al., 2007; Ruiter et al., 2012; Zografos et al., 2013; Duric et al., 2014; Camacho et al., 2012; Liu et al., 2018). In the following, we summarize the main characteristics of three acquisition systems that are close to approach clinical utility. Because the systems are continuously being improved, these descriptions might be outdated. Indicated references in each case, which we used to obtain the information, correspond to the latest publications introducing and describing the systems.

1. QT Scanner 2000 (Lenox et al., 2015; Malik et al., 2018): The QT ultrasound system consists of a transmitter, receiver and three reflection arrays in a U-shaped arrangement, shown in Figure 2.4. The transmitter-receiver arrays are used for transmission tomography. Here, a plane wave is emitted at frequencies ranging from 0.3 to 1.5 $\mathrm{MHz}$, with center frequency at $0.9 \mathrm{MHz}$. After propagating through the tissue, the response is recorded by a 2048-element receiver array ( 8 rows and 256 columns). The size of individual elements is $0.5 \mathrm{~mm}$. Velocity and attenuation reconstructions are 


\section{Ultrasound Computer Tomography}

obtained using 3D non-linear inverse scattering (Wiskin et al., 2017), based on the parabolic approximation to the Helmholtz equation. For reflection images, three reflection arrays are used in B-mode acquisition, with a center frequency of $4 \mathrm{MHz}$. The images are corrected using velocity models obtained from transmission tomography.

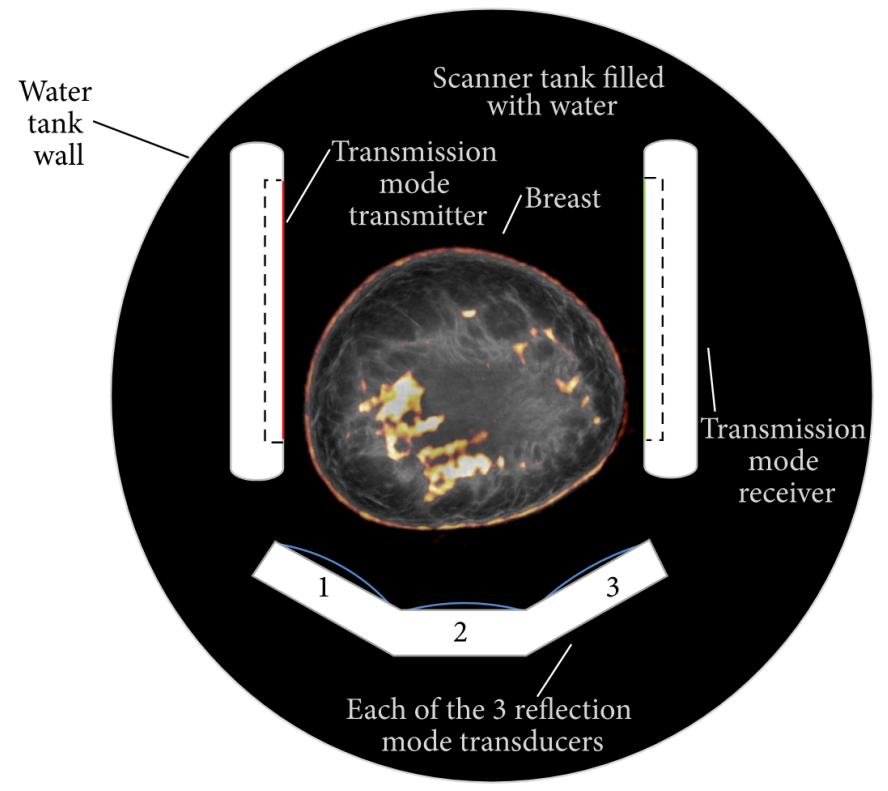

Figure 2.4.: Illustration of the acquisition design for QT Scanner. Modified after Lenox et al. (2015), figure under Creative Commons Attribution License.

2. SoftVue (Duric et al., 2013, 2014): The device consists of a circular transducer array with diameter of $22 \mathrm{~cm}$ and 2048 transducer elements. It can be moved in vertical direction, from the chest wall to the nipple region, acquiring data every $2.5 \mathrm{~mm}$. The transmitted signal has a center frequency of $3 \mathrm{MHz}$, and a bandwidth that provides minimum frequencies of 400-500 kHz (Roy et al., 2016), useful for tomography. It uses synthetic aperture beam-forming techniques for B-mode reflection images (Roy et al., 2013) and frequency-domain waveform tomography to reconstruct 2D images of velocity and attenuation, which are then used to render stiffness images (Duric et al., 2015). The system provides volumetric images of the whole breast by stacking 2D reconstructed coronal slices. For an average patient, the data acquisition takes 1-2 minutes, and the image reconstruction can be done in approximately 30 minutes (Duric and Littrup, 2018).

3. KIT 3D USCT II (Gemmeke et al., 2017): This is a semi-ellipsoidal device, with 26 $\mathrm{cm}$ diameter and $18 \mathrm{~cm}$ height. It has 157 transducer arrays surrounding the breast, each of them containing 4 emitters and 9 receivers of $0.9 \mathrm{~mm}$ size. The aperture can be rotated and vertically translated, which provides additional locations for the 
transducer arrays. An illustration of the KIT 3D USCT is shown in Figure 2.5. Each emitter sends an approximately spherical wave front at center frequency $2.5 \mathrm{MHz}$ with approximately $50 \%$ bandwidth. SAFT is applied to obtain 3D images of reflectivity, and velocity and attenuation are reconstructed using ray-based methods (Dapp et al., 2011). For this device, the data acquisition time is around 10 minutes, and SAFT and transmission reconstructions take approximately 20 and 15 minutes, respectively.

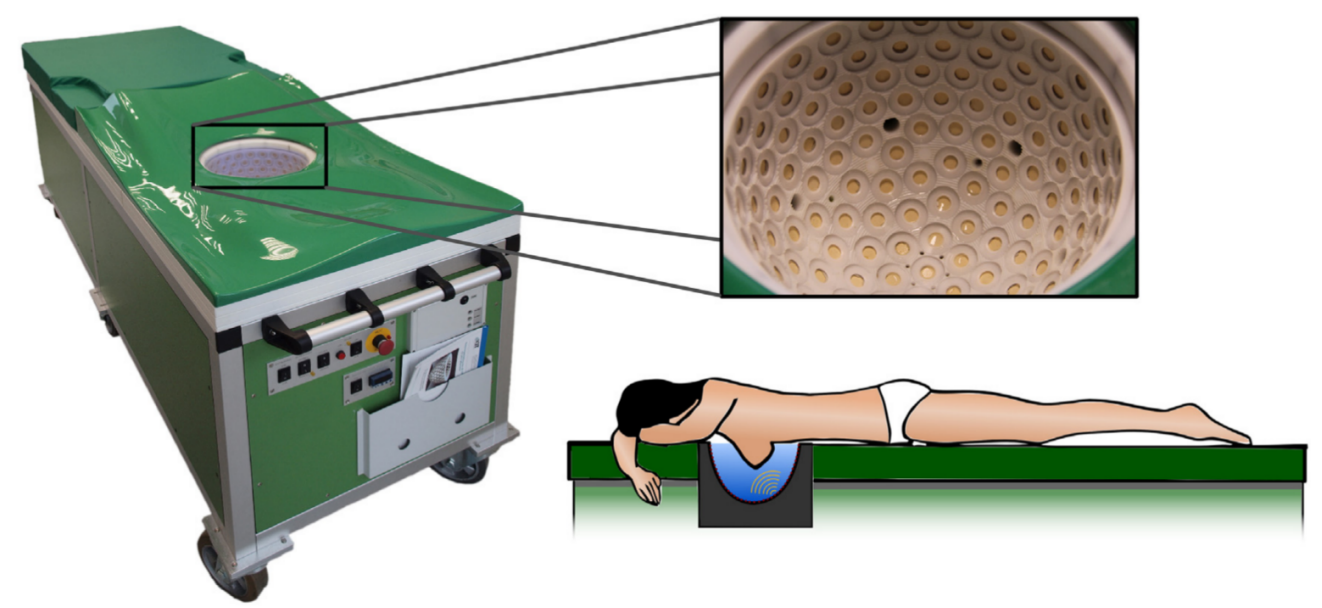

Figure 2.5.: Illustration of the KIT 3D USCT system, with detailed view of the aperture containing the transducer arrays. Reprinted from Gemmeke et al. (2017), with permission from Elsevier.

\subsection{New challenges}

The description of these devices reveals a strong interdependency between reconstruction methods and the design of acquisition systems. In each case, the trade-off between accuracy and practicality is controlled by sacrificing certain aspects. For instance, the practical implementation of waveform tomography is limited to 2D slice-by-slice scanning devices. Here, the cost of numerical wave propagation is reduced by $2 \mathrm{D}$ approximations, and the final image is obtained by stacking reconstructed coronal slices at different elevations. Despite their promising results (Pratt et al., 2007), recent numerical studies have observed the need for 3D considerations to avoid undesirable artifacts in the reconstructions (Wiskin et al., 2013; Goncharsky et al., 2016; Sandhu et al., 2017). Fully 3D devices may overcome these limitations by including out-of-plane scattering and refraction. At present, however, highfrequency transducers are dominating such devices, which excludes the use of waveform tomography.

As we observe, adapting new tomographic methods to the existing acquisition systems is not straightforward and opens new questions: 


\section{Ultrasound Computer Tomography}

1. Could we provide physically consistent 3D reconstructions using the existing sliceby-slice systems?

2. How can we reduce the computational cost of waveform tomography without altering the quality of the reconstructions?

3. Is there any unexplored tomographic method that could benefit from the advantages of both ray-based and waveform tomography? In other words, could we find a method that is as robust and fast as ray-based tomography and still includes finite-frequency effects as waveform tomography?

However, other questions may arise if we formulate the previous observation the other way around, i.e., adapting the existing acquisition systems to new tomographic methods:

1. What are the optimal acquisition designs, in terms of accuracy and cost, for each imaging method?

2. If the ultimate goal is to develop a scanning system for multi-modality imaging, how could we design an acquisition system that is optimal to simultaneously image multiple acoustic parameters?

Acknowledging that reconstruction methods and experimental designs need to be understood in relation to each other, the goal of this thesis is to address the questions formulated above. We moreover acknowledge the work invested by other disciplines in addressing similar questions, and we therefore take the standpoint of the seismologist to bring new answers to the context of medical ultrasound.

In the following chapter we briefly review the advances in seismic tomography during the last decades. We use this to introduce the similarities and differences that exist between both fields, and to understand which methods could be transferred and adapted to the context of USCT. We conclude by outlining the specific contributions made during this thesis. 


\section{Seismic tomography}

The propagation of waves has been widely used to study the internal structure of media that are not accessible to direct observations. Examples of this have been described in previous chapters in the field of medical tomography, but similar approaches can also be found in other disciplines, as for instance in seismology. Seismic tomography aims at creating 2D or 3D images of Earth's interior by exploiting properties of seismic waves. These are generated either naturally by earthquakes and environmental sources, mainly the ocean, or artificially by explosions, specialized trucks, or airgun shots. Recorded at the surface, they provide illumination, in the case of natural sources, down to thousands of kilometers depth. This information has been essential to improve our knowledge of dynamical processes in the Earth's crust and mantle, as well as to understand its evolution.

The origins of seismic tomography in its modern form date back to the mid 1970s, often associated to the work by Aki and Lee (1976), who published the first 3D velocity model using traveltime data of earthquake measurements. This work was based on the assumption of straight ray paths, showing remarkable similarities with the first attempts to USCT by Greenleaf and Johnson (1975) and hence with the development of CT by Hounsfield (1973). Although it is unclear that the latter inspired the foundations of seismic tomography, being situated within the same historical context, both fields were influenced by the similar scientific concerns and knowledge, including resources and methodologies. One of the most influential works may have been the central slice theorem introduced by Radon (1917), who showed the possibility of reconstructing 2D functions (images) from a set of $1 \mathrm{D}$ line integrals.

Unlike USCT, which at that time was considered premature and therefore unnecessary (André et al., 2013; Carson and Fenster, 2009), seismic tomography has continuously advanced since the pioneering work of Aki and Lee (1976). Currently, increasingly sophisticated and powerful methods are used to provide detailed images of subsurface properties at global, continental, regional and local scales. Figure 3.1 shows an example of recent efforts towards a collaborative and evolutionary global tomographic model that incorporates reconstructions at different scales (Fichtner et al., 2018). In the following, we provide a brief historical overview on the main developments in the field of seismic tomography. For an extensive review, we refer the reader to Liu and Gu (2012), Nolet (2008), Iyer and Hirahara (1993) and Rawlinson et al. (2010). 


\section{Seismic tomography}
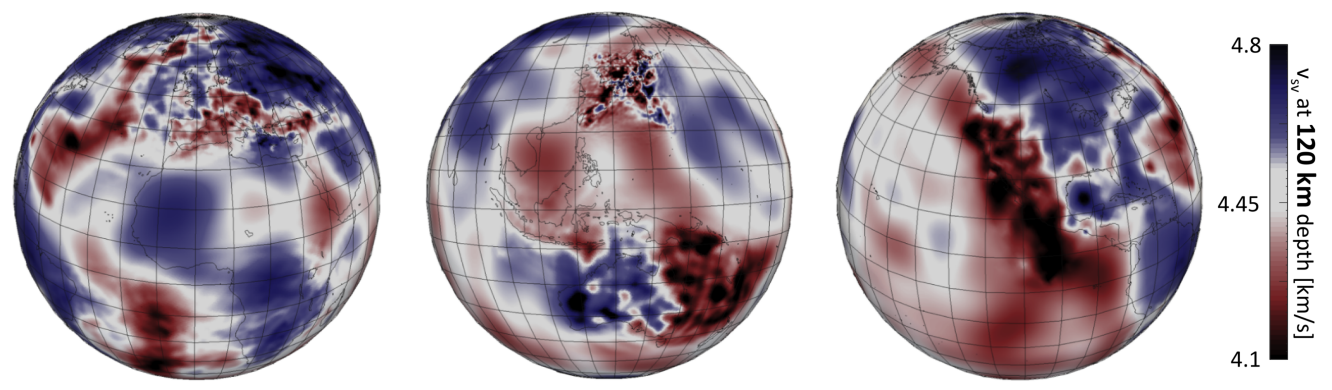

Figure 3.1.: Collaborative seismic earth model. Distribution of vertical shear wave velocity at $120 \mathrm{~km}$ depth. Modified after Fichtner et al. (2018), figure under Creative Commons Attribution License.

\subsection{Brief historical perspective}

The early tomographic studies by Aki et al. (1974) and Aki and Lee (1976) were immediately followed by various applications at regional and local scales using data from large seismic arrays around the world (e.g., Ellsworth and Koyanagi, 1977; Mitchell et al., 1977; Romanowicz, 1979, 1980). By the mid 1980's, high-quality data became available with the development of broadband digital seismometers with large dynamic range, and iterative matrix solvers were introduced to resolve large tomographic systems (Nolet, 1985). This created favourable conditions for global-scale tomographic studies using long-period data, and the first images of the 3D structure of the mantle became possible (Dziewonski, 1984; Dziewonski and Woodhouse, 1987).

The studies mentioned above were exclusively based on the ray-theoretical approaches, and understanding its limitations was a major concern in the following decades (Williamson, 1991; Spetzler et al., 2001). Ray theory assumes the wavelength of the seismic wave to be much smaller than the minimum scale length of heterogeneity, and it therefore imposes limits on resolution of tomographic images derived from it. Subsequent developments in seismic tomography went beyond ray assumptions, in response to rapid improvements in computing power, and considerable efforts were invested into accurately modelling the effects of heterogeneous Earth structure on seismic wave propagation. These include finitefrequency tomography (e.g., Luo and Schuster, 1991; Woodward, 1992; Yomogida, 1992; Marquering et al., 1999; Dahlen et al., 2000; Hung et al., 2000; Friederich, 2003; Montelli et al., 2004; Zhou et al., 2005; Sigloch and Nolet, 2006) and full-waveform tomography (e.g., Gauthier et al., 1986; Tarantola, 1988a; Crase et al., 1990; Pratt, 1999; Chen et al., 2007; Fichtner, 2010; Tape et al., 2010).

Finite-frequency tomography is the natural extension of the ray-based traveltime tomography and uses first-order perturbation theory (Born approximation) to correctly account for the frequency dependence and volumetric sensitivity of traveltime measurements, which 
are related to off-ray-path scattering and diffraction. Consequently, the sensitivity of waves with finite-frequency content is more accurately modelled by this approach as $3 \mathrm{D}$ volumes described by Fresnel zones, rather than infinitely thin rays. The practical implementation of this method has become feasible for global tomography thanks to the efficient implementation of Dahlen et al. (2000), and it is now widely applied (e.g., Yoshizawa and Kennett, 2004; Zhou et al., 2006a; Sigloch et al., 2008; Tian et al., 2009; Yoshizawa and Ekström, 2010).

Full-waveform tomography uses numerical methods to solve the propagation of seismic waves in heterogeneous media, and it therefore has the potential to (1) accurately model all wave phenomena and (2) exploit complete seismic waveforms to improve tomographic resolution. The method is computationally very demanding, but due to recent advances in high-performance computing, it is currently becoming the standard technique in localscale applications (Virieux and Operto, 2009). Similar to continental- and global-scale studies, however, they still remain restricted to long-period data. The challenges arising from the computational complexity of waveform tomography can be illustrated with the first waveform-based global tomography, which took nearly 500 million core hours over a period of 6 years on one the most powerful supercomputers in the world (Bozdag et al., 2016).

\subsection{Context: Similarities and differences}

The historical trends in both seismic tomography and USCT reveal considerable similarities. In both fields, accurate forward modelling of wave propagation is being introduced in order to respond to the demand for high-resolution images. In fact, the first application of waveform tomography in USCT results from an interdisciplinary collaboration between both fields (Pratt et al., 2007). Following this, new collaborative spaces appeared in recent years, which have stimulated fruitful dialogues between both communities and have been essential to understand the particular contexts of each field. This is crucial to explore the extent to which existing solutions and technologies can be transferred from one field to the other. In the following, we summarize the differences and similarities between USCT and seismic tomography. Here we closely follow the recently published work by Pratt (2018).

Similarities between seismic tomography and USCT are undeniable. In both experiments, the propagation of mechanical waves is used to infer the physical properties of media from sparse measurements at the boundary of some region of interest. In such scenarios, the illumination and data coverage is limited, which makes the underlying inverse problem illposed. Therefore, similar difficulties arise in both tomographic problems: appropriate prior information on properties of the media, that translate to suitable regularization strategies, are essential to mitigate the ill-posedness of the inverse problem.

The most obvious difference between both problems is related to the medium in which 


\section{Seismic tomography}

waves propagate. Whereas a human breast can be treated as an acoustic medium in the context of USCT ${ }^{2}$, the Earth behaves elastically and includes a variety of phases and phenomena that cannot be ignored: compressional and shear waves, mode conversions, anisotropic propagation, and surface waves. However, the modelling of acoustic wave propagation is often preferred in seismic exploration. It is computationally less intensive than the complete elastic wave equation, and by applying appropriate strategies that mainly consist in focusing on compressional waves, elastic effects can be suppressed during the inversion (e.g., Shen, 2010; Calderón Agudo, 2018). Currently, acoustic waveform inversion has become a standard practice in oil industry.

The wave equation is scale invariant, and technologies applied in seismic exploration could in principle be transferred to USCT. To understand the difficulties that may arise in this process, we show the scale characteristics of typical experiments in both fields in Table 3.1. Here we specify the highest and lowest frequencies that are used in each case. The most relevant information is the number of wavelengths propagated through the domain of study. As we observe, this number is equal or above 100 for experiments in USCT, showing that current acquisition systems use relatively higher frequencies than the ones in seismic exploration. At such frequencies, (1) waveform inversion becomes sensitive to local minima and cycle-skipping (Gauthier et al., 1986; Bunks et al., 1995), which increases the risk to converge towards potentially meaningless parameter estimations, and (2) numerical wave propagation simulations become computationally very expensive. The latter is undesirable in the practical context of USCT. Here, the acceptable time to solution, from acquiring data to obtaining an image, ranges from real-time to a time span in the order of 10 to 30 minutes, and due to data privacy, the use of external computing facilities is not possible. This differs substantially from seismic tomography, where time and financial constraints are less critical and massive computations are often done on large supercomputers.

\begin{tabular}{|c|ccc|c|}
\cline { 2 - 5 } \multicolumn{1}{c|}{} & \multicolumn{2}{c|}{ Seismic exploration } & \multicolumn{2}{c|}{ Medical ultrasound } \\
\cline { 2 - 5 } \multicolumn{1}{c|}{} & Low frequency & High frequency & Low frequency & High frequency \\
\hline Frequencies & $2.5 \mathrm{~Hz}$ & $25 \mathrm{~Hz}$ & $1 \mathrm{MHz}$ & $3 \mathrm{MHz}$ \\
\cline { 1 - 3 } Domain & $16 \mathrm{~km}$ & $16 \mathrm{~km}$ & $15 \mathrm{~cm}$ & $15 \mathrm{~cm}$ \\
\cline { 1 - 4 } Velocity & $4000 \mathrm{~m} / \mathrm{s}$ & $4000 \mathrm{~m} / \mathrm{s}$ & $1500 \mathrm{~m} / \mathrm{s}$ & $1500 \mathrm{~m} / \mathrm{s}$ \\
\cline { 1 - 4 } Wavelength & $1.6 \mathrm{~km}$ & $0.16 \mathrm{~km}$ & $1.5 \mathrm{~mm}$ & $0.5 \mathrm{~mm}$ \\
\cline { 1 - 4 } \# wavelengths & 10 & 100 & 100 & 300 \\
\hline
\end{tabular}

Table 3.1.: A comparison of scale characteristics in typical experiments of exploration-scale seismic tomography and USCT. In both fields, we specify the low and high frequencies that are typically used.

\footnotetext{
${ }^{2}$ Although the elastic effects are rather weak in breast tissue (reported shear wave velocities are on the order of $10 \mathrm{~m} / \mathrm{s}$ (e.g., Athanasiou et al., 2010)), they may have an important role in other imaging modalities, for instance, in elastography (Berg et al., 2012).
} 
In principle, the most straightforward comparison for USCT is with seismic exploration. Both fields image local-scale structures, looking for specific targets, either hydrocarbon reservoirs or cancerous tissue, and the design of the acquisition system is fully controlled by those performing the experiment. However, the properties of breast tissue may show more similarities with those appearing in global tomography. Figure 3.2(a) illustrates the velocity variations in a realistic breast phantom generated from magnetic resonance data (Lou et al., 2017). As we observe, breast tissue shows relatively low variations that are on the order of 5-10\%. This suggests similarities with the Earth's mantle [see Figure 3.2(b)], a domain in which approximations of the physical modelling are extensively used to obtain detailed images (e.g., Hosseini et al., 2018; Auer et al., 2014; Simmons et al., 2010, 2015). Exploring technologies and knowledge from global tomography to image breast tissue with ultrasound may therefore be of significant value, and it will be addressed in this thesis.

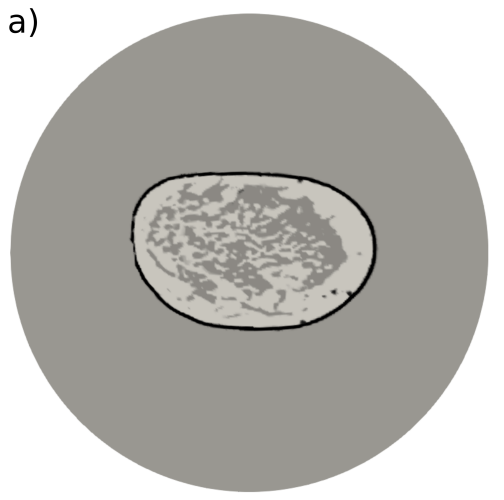

b) Hosseini2016

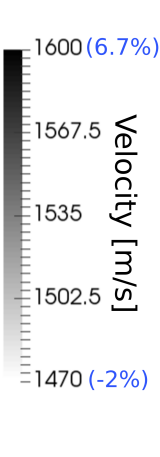

$600 \mathrm{~km}$

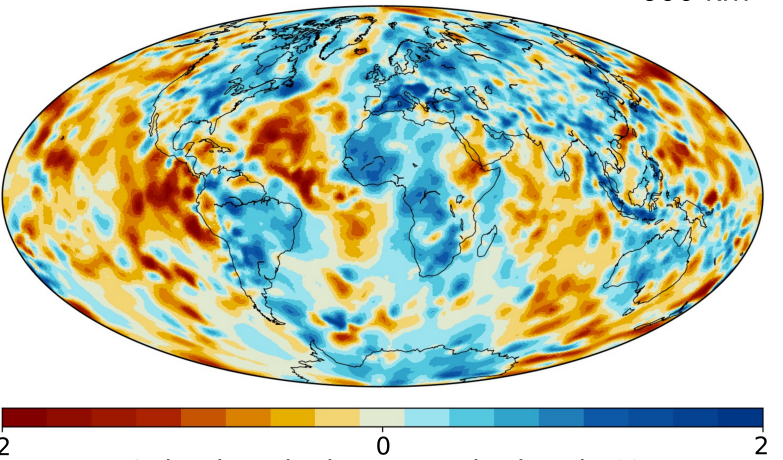

Seismic velocity anomaly $\mathrm{dv} / \mathrm{v}$ in \%

Figure 3.2.: (a) Coronal slice of a synthetic phantom generated from magnetic resonance data (Lou et al., 2017). It illustrates the velocity variations in breast tissue. Relative velocity variations with respect to water $(1500 \mathrm{~m} / \mathrm{s})$ are indicated in blue in the colorbar. (b) Core-diffracted P wave global velocity model of Earth at $600 \mathrm{~km}$ depth (Hosseini, 2016). Image generated using SubMachine (Hosseini et al., 2018).

\subsection{Lessons from seismology}

As we observed, seismology and medical ultrasound share many similarities that allow knowledge transfer from one field to the other. However, major differences reside in the tradeoff between accuracy and practicality that medical practice demands. To be meaningful, the transferred knowledge must recognize these differences, selecting and adapting the methods accordingly. In general, we may ask, what strategies are used in seismology to accurately image the structure of the media in a computationally feasible manner?; and in particular, which of these experiences could provide us answers to the specific questions about USCT formulated in Section 2.3? 


\section{Seismic tomography}

Some of these questions were related to the design of the acquisition system. Current designs might not be optimal for every imaging technique, as we concluded from Table 3.1. In seismic exploration, where enormous amounts of money are invested every year by industry in seismic surveys ${ }^{3}$, we often find similar concerns about cost-efficient designs that provide as much information as possible about properties of the media. In this section, we will briefly review the experimental optimization approaches that have been developed or adapted to seismology.

Redesigning the existing acquisition systems in USCT, however, would require a significant investment, and exploring new imaging methods may be more desirable. In particular, we wondered in Section 2.3 about the existence of any method that could benefit from the strengths of both ray-based (fast) and waveform (accurate) tomography. As mentioned before, finite-frequency tomography is widely used in global- and continental-scale, as a computationally tractable counterpart of waveform tomography in higher frequency domains. In the second half of this section, we will review in more detail the development of this method in seismology.

\subsubsection{Optimal Experimental Design}

Since the 1990's, various studies have published systematic frameworks to optimize geophysical surveys, including seismic tomography experiments (Maurer et al., 2010). These are known as Optimal Experimental Design (OED) methods, and the goal is to provide an acceptable survey design acquiring data that best resolve specific parameters of interest. Because this goal is quite general, OED methods have found diverse applications. For instance, it has been applied to (1) determine the optimal locations of seismometers to reduce the uncertainty in locating earthquakes (Rabinowitz and Steinberg, 1990), (2) optimize the receiver configuration in microseismic monitoring surveys (Curtis et al., 2004) and (3) select the optimal frequencies for frequency-domain waveform inversion in crosshole surveys (Maurer et al., 2009).

The quality of a design, which is the quantity to optimize, is usually formulated in terms of the expected uncertainties in model parameters post-reconstruction. This depends on the model-data relationship (Curtis, 2004a,b), i.e., the reconstruction method expected to be applied post-acquisition, and OED methods can therefore be seen as an optimization problem that lies on top of another inverse problem. Typically, linearizing assumptions are used to access the expected uncertainties without reconstructing the parameters in each step (Curtis, 2004a; Maurer et al., 2009, 2017). However, the resulting optimization problem still remains non-linear, and a variety of global optimization algorithms have been used for this purpose (Hardt and Scherbaum, 1994; Curtis, 1999a; Ajo-Franklin, 2009; Roux

\footnotetext{
${ }^{3}$ For example, BP (British Petroleum) company had an expenditure of $\sim 2000$ million USD in 2017 for the exploration and evaluation of oil and natural gas resources (BP Annual Report and Form 20-F 2017).
} 
and Garcia, 2014). When the number of design parameters is large, global optimization becomes prohibitive, and methods to sequentially optimize the experimental setup have been suggested as alternative (Guest and Curtis, 2009; Maurer et al., 2017). These methods are particularly interesting, since they provide benefit-cost curves that quantify the information gain versus the cost related to different experimental configurations. These curves are useful to identify redundancies in the measurements, and by doing so, maximize the benefit-cost ratio of an experiment (Maurer et al., 2017).

Currently, to the best of our knowledge, USCT lacks systematic frameworks to optimize the acquisition designs based on the expected posterior uncertainties in model parameters. Typically, approaches that homogenize the data coverage have been applied, either following the Nyquist-Shannon sampling theorem for uniform transducer arrays (Simonetti et al., 2007) or geometrical approaches for sparse transducer arrays (Gemmeke et al., 2014). In this context, OED method could provide a valuable contribution to both optimally design new acquisition system for multi-modality imaging and assess the quality and cost of the currently existing ones.

\subsubsection{Finite-frequency tomography}

The seismic imaging community has for a long time recognized that actual seismic waves are band-limited and affected by the medium in the neighbourhood of the geometric ray path. Ray theory therefore becomes insufficient to accurately describe the complexity of seismic wave propagation, including traveltime observations. As a consequence, several attempts have been made to incorporate finite-frequency effects into traveltime tomography. For example, Luo and Schuster (1991) first introduced the cross-correlation traveltime-shift misfit functional into the framework of waveform tomography, and Woodward (1986, 1992) applied diffraction tomography to derive the 'wavepaths' (band-limited ray paths), i.e., the Fréchet derivatives of band-limited traveltimes with respect to velocity perturbations. These works established the foundations of what later on will be called finite-frequency tomography.

The term finite-frequency tomography acknowledges the non-infinite nature of waves in both the measurement and the computation of sensitivities. In the last two decades, however, it has been mostly used to refer to tomography that (1) uses first-order perturbation theory to account for scattering and diffraction effects, and (2) considers cross-correlation of signals to compute traveltime measurements. The method was introduced to global tomography by Marquering et al. (1999) and Dahlen et al. (2000), who emphasized the fact that traveltimes are insensitive to velocity variations along the geometric ray path (previously observed by Woodward $(1986,1992)$ and Yomogida (1992)) and used the term "banana doughnut" kernel to describe this phenomenon. Since then, numerous studies have shown successful applications of finite-frequency tomography at regional (Friederich, 2003; 


\section{Seismic tomography}

Yoshizawa and Kennett, 2004; Sigloch et al., 2008) and global scales (Montelli et al., 2004; Zhou et al., 2006b).

Compared to ray theory, the finite-frequency method increases the amount of useful information extracted from the signals, while still being a linearized problem, valid for velocity contrasts of up to $10 \%$ with respect to a background model (Mercerat and Nolet, 2012). As observed in Figure 3.2a, this condition is guaranteed in breast tissue. The increased information is a consequence of considering cross-correlation traveltime measurements, which are inherently frequency-dependent (Tong et al., 1998a), i.e., they depend on the frequency content of the cross-correlated pulses. This is consistent with the traveltime estimations in USCT, in which observed signals are cross-correlated with calibration data in water to compute the traveltime differences. New developments in finite-frequency tomography have shown that the amount of available information can be further increased. This includes finite-frequency tomography for amplitudes (Dahlen and Baig, 2002; Sigloch and Nolet, 2006) and multiple-frequency finite-frequency approaches (Sigloch et al., 2008; Hung et al., 2004; Hosseini, 2016). The latter takes advantage of the frequency content of traveltime measurements to enlarge the dataset and, therefore, improve tomographic resolution.

To the best of our knowledge, the potential of finite-frequency tomography has not been explored in the context of USCT. It can offer a computationally practical approach to (1) incorporate finite-frequency effects in the existing acquisition systems that still rely on high-frequency transducers and (2) provide physically consistent 3D images in slice-by-slice systems. This is naturally obtained by correctly accounting for volumetric sensitivity of traveltime measurements.

\subsection{Thesis: Objective and Outline}

The objective of this thesis is now apparent. We will answer the questions formulated in Section 2.3 introducing and adapting optimal experimental design method and finitefrequency tomography to imaging breast tissue with ultrasound. The overall goal of our work is to focus on practical solutions that are valid for the current computational and technological resources, particularly those available for clinical practice. This therefore excludes waveform tomography from the research topics addressed during this thesis, mainly for two reasons: (1) it is still from hard to impossible to have access to data that fall into the regime of cost-effective waveform tomography. These data may exist but are in possession of private companies, which restricts their use in public research institutions; (2) waveform tomography is still far from being practical in 3D domains using current acquisition designs, and it therefore represents a long-term research topic that diverges from the overall goal of this thesis. In any case, we will address few aspects on uncertainty quantification for waveform tomography, useful for optimal experimental design methods.

The rest of the thesis is organized as follows. Chapter 4 introduces and describes the 
forward and inverse theory underlying to the image reconstruction methods considered within our studies. We specifically focus on (1) problems where the relationship between observables and unknown parameters is linear, and (2) reconstruction strategies that have been studied and implemented during this thesis. In particular, we employ an illustrative toy example to visualize many aspects of the inverse theory that are described.

In Chapter 5, we extend the collection of imaging techniques and present a new transmission tomography method for medical ultrasound. Here, traveltime finite-frequency tomography is introduced and adapted to USCT, and we validate its applicability for 3D reconstructions in slice-by-slice acquisition systems using lab measurements. We moreover discuss an extension of the method for amplitude tomography. This chapter is adapted from:

N. Korta Martiartu, C. Boehm and A. Fichtner (2019). 3D Wave-Equation-Based Finite-Frequency Tomography for Ultrasound Computed Tomography. IEEE Transactions on Ultrasonics, Ferroelectrics, and Frequency Control. Submitted for publication. Preprint available at arXiv:1908.03302.

Once reconstruction methods are well understood, we proceed with the analysis of the experimental designs, i.e., we study the influence of transducer arrangements in the estimation of tissue properties. Chapter $\mathbf{6}$ is dedicated to introduce optimal experimental design methods. The main aspects described here are (1) quantities estimating the quality of particular designs, and (2) optimization strategies that solve the involved non-linear minimization problem. The latter includes global and sequential techniques that are applied to optimally position transducers for transmission tomography in 3D. This chapter is adapted or includes contributed parts from:

N. Korta Martiartu, C. Boehm, N. Vinard, I. Jovanović Balic and A. Fichtner (2017). Optimal experimental design to position transducers in ultrasound breast imaging. Proceedings SPIE 10139, Medical Imaging 2017: Ultrasonic Imaging and Tomography, 10139, 101390M.

H. Maurer, A. Nuber, N. Korta Martiartu, F. Reiser, C. Boehm, E. Manukyan, C. Schmelzbach, and A. Fichtner (2017). Optimized experimental design in the context of seismic full waveform inversion and seismic waveform imaging. Advances in Geophysics, vol. 58 , pp. $1-45$.

An ideal USCT acquisition system must account for multi-modality imaging. We therefore explore, in Chapter 7, the flexibility and potential of OED to include both transmission and reflection data, and to use either ray-based or wave-based methods for the reconstructions. Here, an intuitive toy example will help us to understand the efficiency of the joint 


\section{Seismic tomography}

optimization approach we present, and this is then validated using $2 \mathrm{D}$ experimental data. This chapter is adapted from:

N. Korta Martiartu, C. Boehm, V. Hapla, H. Maurer, I. Jovanović Balic and A. Fichtner (2019). Optimal experimental design for joint reflection-transmission ultrasound breast imaging: from ray- to wave-based methods. The Journal of the Acoustical Society of America, 146(2), 1252-1264.

Finally, we draw few concluding remarks in Chapter 8, where we connect again both seismology and medical ultrasound to foster future interdisciplinary collaborations that may benefit imaging methods on all scales. 


\section{Image reconstruction}

"The fundamental laws of physics do not describe true facts about reality. Rendered as descriptions of facts, they are false; amended to be true, they lose their fundamental, explanatory force."

- Nancy Cartwright, How the Laws of Physics Lie (1983)

This chapter introduces the tomographic and imaging techniques that are considered in this thesis and is organized in two parts. The first part introduces the forward problem corresponding to each imaging method. That is, the physical model used to predict the observed data from specific acoustic tissue properties (model parameters). We start from a compact formulation that unifies the notation for every imaging approach, and we then provide explicit expressions of the forward operator (parameter-to-observable map) in each case. The second part uses this general notation to provide a common framework for the inverse problems arising in each technique. The inverse problem is the mathematical approach of inferring unknown model parameters from observations of some data indirectly related to them through the forward problem. Here we focus on linear(ized) forward and inverse problems, i.e., problems in which the relationship between the observations and unknown parameters can be considered linear to certain extent. These include (1) traveltime tomography using either straight rays or (2) finite frequency sensitivity kernels, (3) the B-mode imaging technique, and (4) reverse-time migration. All these methods have been studied and implemented as part of this thesis. Although our work also has considered waveform inversion several times, we intentionally exclude non-linear approaches from this chapter to simplify the notation. Instead, we provide a linearized forward formulation for waveform tomography that is useful to approximate the posterior covariance operator required for the OED methods in Chapters 6 and 7. Interested readers can find a comprehensive description of waveform modelling and inversion in Fichtner (2010).

\subsection{Forward theory}

The goal in USCT is to estimate the acoustic properties of breast tissue $\mathbf{m}$ from the observations of the space- and time-dependent pressure wavefield $p(\mathbf{x}, t)$ that are recorded by an experimental setup s surrounding the breast. The physics governing USCT experiments, defined in the time interval $\left[t_{0}, t_{1}\right]$ and spatial domain $\Omega \subset \mathbf{R}^{3}$ with boundary $\partial \Omega$, can be 


\section{Image reconstruction}

described by the acoustic wave equation

$$
\frac{1}{\rho(\mathbf{x}) c^{2}(\mathbf{x})} \partial_{t}^{2} p(\mathbf{x}, t)-\nabla \cdot\left(\frac{1}{\rho(\mathbf{x})} \nabla p(\mathbf{x}, t)\right)=\frac{1}{\rho(\mathbf{x})} f(\mathbf{x}, t), \quad \text { in } \Omega \times\left[t_{0}, t_{1}\right]
$$

with the initial conditions

$$
p(\mathbf{x}, 0)=0,\left.\quad \partial_{t} p(\mathbf{x}, t)\right|_{t=0}=0, \quad \text { in } \Omega,
$$

and boundary conditions

$$
\mathbf{n}(\mathbf{x}) \cdot \nabla p(\mathbf{x}, t)=0 \quad \text { on } \partial \Omega \times\left[t_{0}, t_{1}\right] .
$$

Here $f$ is the external source generated from emitting transducers, $\mathbf{n}(\mathbf{x})$ is the normal direction to $\partial \Omega$ pointing outwards, and the parameters $\rho$ and $c$ represent the density and velocity distribution of breast tissue, respectively, being $\mathbf{m}=[c(\mathbf{x}) ; \rho(\mathbf{x})]$. The interaction of the pressure field with breast tissue is recorded at receiver locations specified by the scanning device $\mathbf{s}$. Here, $\mathbf{s}$ indicates, for instance, the locations of the active transducers. We denote by $p\left(\mathbf{x}_{r}, t ; \mathbf{x}_{s}\right)$ the pressure field generated by an emitter at $\mathbf{x}_{s}$ and recorded at receivers $\mathbf{x}_{r}$, and in general, we take specific parts from this time series to define the observables $\mathbf{d}_{\text {obs }}$. For instance, $\mathbf{d}_{\text {obs }}$ contains the recorded times of the first arrivals.

Although the acoustic wave equation (4.1) represents a highly accurate attempt of describing observable-parameter relationship, a variety of other physical models are typically used in USCT that approximate Equation (4.1) with different degrees of accuracy. Using compact notation, the physical model that predicts observable parameters $\mathbf{d}$ from model parameters $\mathbf{m}$ can be expressed by the forward operator $\mathbf{F}$ as

$$
\mathbf{d}=\mathbf{F}(\mathbf{m} ; \mathbf{s})+\boldsymbol{\epsilon}
$$

where the vector $\epsilon$ accounts for the measurement noise that we in general assume to be Gaussian with zero-mean and covariance matrix $\boldsymbol{\Gamma}_{\text {noise }}$. In Sections 4.1.1 and 4.1.2, we discuss specific formulations for the forward operator of the imaging techniques considered here. Note the differences between $\mathbf{d}$ and $\mathbf{d}_{\text {obs }}$ introduced in Equation (4.4). The former represents our predictions about the observables, while the latter indicates the empirically measured observations.

In specific situations, the non-linearities of $\mathbf{F}(\mathbf{m} ; \mathbf{s})$ with respect to $\mathbf{m}$ are not too severe, and in particular, we may assume that the acoustical parameters show relatively small variations with respect to some prior knowledge $\mathbf{m}_{\text {prior }}$. This can be, for instance, the medium in which calibration data are recorded. In such cases, the forward operator can 
be linearized as

$$
\mathbf{F}(\mathbf{m} ; \mathbf{s}) \approx \mathbf{F}\left(\mathbf{m}_{\text {prior }} ; \mathbf{s}\right)+\left.\mathbf{F}^{\prime}(\mathbf{m} ; \mathbf{s})\right|_{\mathbf{m}=\mathbf{m}_{\text {prior }}}\left(\mathbf{m}-\mathbf{m}_{\text {prior }}\right)
$$

where higher-order model perturbation terms have been neglected. Here, $\mathbf{F}^{\prime}$ is the first derivative of the forward operator with respect to $\mathbf{m}$, i.e., the Jacobian operator. For notational simplicity, we will continue using $\mathbf{F}^{\prime}(\mathbf{s})$ to express $\mathbf{F}^{\prime}(\mathbf{m} ; \mathbf{s})$ evaluated at $\mathbf{m}_{\text {prior }}$.

Upon inserting Equation (4.5) into Equation (4.4), the forward problem becomes

$$
\delta \mathbf{d}=\mathbf{F}^{\prime}(\mathbf{s}) \delta \mathbf{m}+\boldsymbol{\epsilon},
$$

where $\delta \mathbf{d}=\mathbf{d}-\mathbf{F}\left(\mathbf{m}_{\text {prior }} ; \mathbf{s}\right)$ and $\delta \mathbf{m}=\mathbf{m}-\mathbf{m}_{\text {prior }}$. Equation (4.6) establishes a linear relationship between parameters $\delta \mathbf{m}$ and observables $\delta \mathbf{d}$, and it describes, in abstract notation, the physical modelling involved in any imaging technique considered in this study. In the following, we provide explicit expressions of the Jacobian operator $\mathbf{F}^{\prime}$ for each specific method. We divide them in two blocks to differentiate the methods using either the infinite- or finite-frequency assumptions. We do not consider imaging techniques that reconstruct the density distribution, and this is therefore assumed as constant in our derivations. Instead, we mainly focus on retrieving the velocity and reflectivity distribution of the breast tissue. As we will observe, the latter can be expressed in terms of relative velocity perturbations. For every approach, we moreover specify the link to the notation in Equation (4.6).

\subsubsection{Ray-based methods: Infinite-frequency}

\subsubsection{Straight-ray tomography}

Traveltime tomography: velocity

For the velocity reconstructions, first-arrival times are often approximated by straightray models (Li et al., 2009; Dapp et al., 2011; Gemmeke et al., 2017). Here, changes in the traveltime $\delta \mathbf{d}_{i}=\delta T\left(\mathbf{x}_{s}{ }^{i}, \mathbf{x}_{r}{ }^{i}\right)$ of the $i$-th ray that travels from the emitter at $\mathbf{x}_{s}{ }^{i}$ to the receiver at $\mathbf{x}_{r}{ }^{i}$ are related to the velocity perturbations as

$$
\delta T\left(\mathbf{x}_{s}{ }^{i}, \mathbf{x}_{r}{ }^{i}\right)=-\int_{\mathrm{ray}_{i}} \frac{\delta c(\mathbf{x}(l))}{c_{0}{ }^{2}} d l \approx-\sum_{j=1}^{N} \frac{l_{i j}}{c_{0}^{2}} \delta c_{j},
$$

where $\mathbf{x}(l)$ is position along the ray-path in terms of arclength $l$. In the discrete form, we assume that the region of interest (ROI) has been partitioned into $N$ cells with constant velocity. Then, $l_{i j}$ represents the length of the $i$-th ray in the $j$-th cell, $\delta \mathbf{m}_{j}=\delta c_{j}$ being the corresponding velocity difference with respect to some background velocity $c_{0}$. Here, $c_{0}$ refers to the homogeneous medium in which calibration data is recorded, i.e., water. If the 


\section{Image reconstruction}

experiment has collected a total of $M$ measurements, the Jacobian operator is a matrix of size $M \times N$ with the elements given by

$$
\mathbf{F}_{i j}^{\prime}=-\frac{l_{i j}}{c_{0}^{2}}
$$

Equation (4.7) introduces a linearization step that is useful to connect the notation with the rest of the imaging techniques. In practice, however, this may be unnecessary if we consider different paramterizations. In case we reconstruct slowness $s(\mathbf{x})=\frac{1}{c(\mathbf{x})}$ instead of velocity, the forward problem is linear,

$$
T\left(\mathbf{x}_{s}{ }^{i}, \mathbf{x}_{r}{ }^{i}\right)=\int_{\text {ray }_{i}} \frac{d l}{c(\mathbf{x}(l))}=\int_{\operatorname{ray}_{i}} s(\mathbf{x}(l)) d l \approx \sum_{j=1}^{N} l_{i j} s_{j},
$$

with the forward matrix given by

$$
\mathbf{F}_{i j}=l_{i j}
$$

Amplitude tomography: attenuation

Most of the work presented in this thesis does not consider attenuation as parameter to infer, and this is the reason why the acoustic wave equation (4.1) is stated for loss-less media. However, in the particular case of straight-ray tomography, Equation (4.9) can easily be modified to reconstruct the attenuation distribution in breast tissue, while reusing the same algorithm.

In soft tissues, it has been empirically shown that the attenuation of ultrasound signals $\beta$ increases linearly with frequency $\omega$ (Kak and Dines, 1978). In this case, the amplitude spectrum $|S(\omega)|_{i}$ of the first-arrival pulse of a signal emitted from $\mathbf{x}_{s}^{i}$ and received at $\mathbf{x}_{r}^{i}$ can be expressed as ( Li et al., 2008; Peterlik et al., 2008)

$$
|S(\omega)|_{i}=\left|S_{0}(\omega)\right|_{i} \exp \left[-\hat{\beta}\left\|\mathbf{x}_{s}^{i}-\mathbf{x}_{r}^{i}\right\| \frac{\omega}{2 \pi}\right] .
$$

Here, $\left|S_{0}(\omega)\right|_{i}$ is the spectrum corresponding to the background model (water), and $\hat{\beta}$ denotes the mean attenuation coefficient along the propagation distance $\left\|\mathbf{x}_{s}^{i}-\mathbf{x}_{r}^{i}\right\|$. By assuming straight rays, we can express Equation (4.11) in terms of the local attenuation distribution as

$$
|S(\omega)|_{i}=\left|S_{0}(\omega)\right|_{i} \exp \left[-\frac{\omega}{2 \pi} \int_{\text {ray }_{i}} \beta(\mathbf{x}(l)) d l\right],
$$

and by reformulating it, the forward problem becomes linear

$$
\frac{2 \pi}{\omega} \log \left[\frac{\left|S_{0}(\omega)\right|_{i}}{|S(\omega)|_{i}}\right]=\int_{\mathrm{ray}_{i}} \beta(\mathbf{x}(l)) d l \approx \sum_{j=1}^{N} l_{i j} \beta_{j} .
$$


This is the linearized forward problem for amplitude tomography. By linking it to the notation in Equation (4.6), the forward matrix is the same as in Equation (4.10), and the observables and parameters are $\delta \mathbf{d}_{i}=\frac{2 \pi}{\omega} \log \left[\frac{\left|S_{0}(\omega)\right|_{i}}{|S(\omega)|_{i}}\right]$ and $\delta \mathbf{m}_{j}=\beta_{j}$, respectively.

\subsubsection{B-mode technique: reflectivity}

The B-mode technique is widely used to image the reflectivity of breast tissue (e.g Gemmeke et al., 2017; Schmidt et al., 2011). Based on the delay-and-sum principle, the image is constructed by focusing the received energy into the scatterer locations. The method assumes single scattering, and computes the traveltimes $T$ using straight rays, similar to Equation (4.9). The imaging condition for the reflectivity distribution $I(\mathbf{x})$ is

$$
I(\mathbf{x})=\sum_{i} E\left(\mathbf{x}_{r}^{i}, t=T\left(\mathbf{x}_{s}^{i}, \mathbf{x}\right)+T\left(\mathbf{x}, \mathbf{x}_{r}^{i}\right) ; \mathbf{x}_{s}^{i}\right)
$$

where $E$ is the envelope of the recorded signal $p\left(\mathbf{x}_{r}, t ; \mathbf{x}_{s}\right)$ that we can compute using Hilbert's transform. The summation over $i$ indicates all emitter-receiver combinations. Each time instant of the recorded signal is transformed to a space location using the emitter-scatterer-receiver geometry, where each grid point is a possible scatterer location. Taking the envelope may limit the resolution of the reflection images and other methods have been suggested that sum either time shifted recorded signals or a matched filtered version of it (Stotzka et al., 2005; Jensen et al., 2006, e.g.,).

Equation (4.14) approximates the unknown reflectivity distribution, and it is a proxy to the solution of the underlying inverse problem. To obtain more accurate images, different studies have reformulated Equation (4.14) into a forward and inverse problem (Lavarello et al., 2006). In the following, we derive the forward problem of the B-mode technique. This will be particularly useful for the optimal experimental design study in Chapter 7, where we expect equivalent solutions for both approaches. In our derivation, we drop the dependency on the emitter-receiver combination $i$ for clarity.

We first assume an unbounded homogeneous medium with velocity $c_{0}$. For a point source $f(t) \delta\left(\mathbf{x}-\mathbf{x}_{s}\right)$ of duration $\tau$, the pressure field $p_{0}(\mathbf{x}, t)$ is analytically expressed using the representation theorem (e.g., Aki and Richards, 2002) as

$$
p_{0}(\mathbf{x}, t)=\int_{V} \int_{0}^{\tau} G\left(\mathbf{x}, t ; \mathbf{x}^{\prime}, t^{\prime}\right) f\left(t^{\prime}\right) \delta\left(\mathbf{x}^{\prime}-\mathbf{x}_{s}\right) d t^{\prime} d \mathbf{x}^{\prime}=\frac{1}{4 \pi\left\|\mathbf{x}-\mathbf{x}_{s}\right\|} f\left(t-\frac{\left\|\mathbf{x}-\mathbf{x}_{s}\right\|}{c_{0}}\right),
$$

where $G\left(\mathbf{x}, t ; \mathbf{x}_{s}, t^{\prime}\right)$ is the $3 \mathrm{D}$ Green's function for the unbounded medium $c_{0}$,

$$
G\left(\mathbf{x}, t ; \mathbf{x}^{\prime}, t^{\prime}\right)=\frac{1}{4 \pi\left\|\mathbf{x}-\mathbf{x}^{\prime}\right\|} \delta\left(t-t^{\prime}-\frac{\left\|\mathbf{x}-\mathbf{x}^{\prime}\right\|}{c_{0}}\right) .
$$

Now, assume that the medium is slightly perturbed to $c_{0}+\delta c(\mathbf{x})$. The new wavefield 


\section{Image reconstruction}

can be expressed as $p_{0}+\delta p$, where $\delta p$ is the scattered field due to the perturbation $\delta c$ and defines our observables, i.e., $\delta \mathbf{d}_{i}=\delta p\left(\mathbf{x}_{r}^{i}, t ; \mathbf{x}_{s}^{i}\right)$. Under the Born approximation, the acoustic wave equation that relates $\delta p$ and $\delta c$ is given by

$$
\left(\frac{1}{c_{0}^{2}} \partial_{t}^{2}-\nabla^{2}\right) \delta p(\mathbf{x}, t)=\frac{2 \delta c(\mathbf{x})}{c_{0}^{3}} \partial_{t}^{2} p_{0}(\mathbf{x}, t)
$$

This equation and subsequent derivations are reviewed in detail in Appendix A.

The scattered field $\delta p$ is generated by secondary sources exited due to the interaction of the pressure field $p_{0}$ with the velocity perturbation $\delta c$. Here, $\delta \mathbf{m}=I(\mathbf{x})=\frac{2 \delta c(\mathbf{x})}{c_{0}}$ is the unknown reflectivity distribution. Similar to Equation (4.15), the solution $\delta p=\delta p(\mathbf{x}, t)$ can be expressed as (Tarantola, 1984)

$$
\delta p=\frac{1}{16 \pi^{2} c_{0}^{2}} \int_{V} \frac{1}{\left\|\mathbf{x}-\mathbf{x}^{\prime}\right\|\left\|\mathbf{x}^{\prime}-\mathbf{x}_{s}\right\|} \delta\left(t-\frac{\left\|\mathbf{x}-\mathbf{x}^{\prime}\right\|+\left\|\mathbf{x}^{\prime}-\mathbf{x}_{s}\right\|}{c_{0}}\right) I\left(\mathbf{x}^{\prime}\right) d \mathbf{x}^{\prime} * \partial_{t}^{2} f(t)
$$

where $V$ is the volume of the ROI and $*$ indicates the convolution operation. This equation describes the forward problem related to the B-mode imaging. Typically, to obtain sharper reflectivity images, the source-time function is deconvolved from the measured signals, or alternatively used for matched filtering, and the transmitted information is muted from them. We denote as $\left[t_{0}, t_{1}\right]$ the time interval of the scattered information in the recorded signals, $t_{1}$ being the recording length. Therefore, for the measurement $i$ that combines the emitter $\mathbf{x}_{s}^{i}$ and the receiver $\mathbf{x}_{r}^{i}$, the elements of the discrete Jacobian are

$$
\mathbf{F}_{i j}^{\prime}=\frac{1}{16 \pi^{2} c_{0}^{2}} \Delta V_{j} \frac{1}{\left\|\mathbf{x}_{r}^{i}-\mathbf{x}_{j}\right\|\left\|\mathbf{x}_{j}-\mathbf{x}_{s}^{i}\right\|} \delta_{\left[t_{0}, t_{1}\right]}\left(\tau_{j}\right)
$$

where $\Delta V_{j}$ is the volume of the $j$-th cell located at $\mathbf{x}_{j}$, and

$$
\delta_{\left[t_{0}, t_{1}\right]}\left(\tau_{j}\right)=\left\{\begin{array}{ll}
1, & \tau_{j} \in\left[t_{0}, t_{1}\right] \\
0, & \tau_{j} \notin\left[t_{0}, t_{1}\right]
\end{array} \quad \text { with } \quad \tau_{j}=\frac{\left\|\mathbf{x}_{r}^{i}-\mathbf{x}_{j}\right\|+\left\|\mathbf{x}_{j}-\mathbf{x}_{s}^{i}\right\|}{c_{0}} .\right.
$$

\subsubsection{Wave-based methods: Finite-frequency}

Ultrasonic sources used in USCT are band-limited, and finite-frequency effects may be important. Recently, wave equation based approaches have been proposed to improve the resolution of the USCT images. These include waveform tomography for velocity reconstructions (Pratt et al., 2007; Matthews et al., 2017; Pérez-Liva et al., 2017a; Boehm et al., 2018) and reverse-time migration (RTM) for reflectivity images (Roy et al., 2016). 


\subsubsection{Traveltime waveform tomography: velocity}

Waveform inversion is often stated as a non-linear least-squares problem, in which we minimize the $L_{2}$-distance between the observed and modelled signals (Roy et al., 2010; Goncharsky et al., 2016; Boehm et al., 2018). Waveform differences, however, are highly non-convex with respect to the model parameters, and convergence towards the global minimum may be difficult. In seismic tomography, a variety of misfit functionals with improved convexity have been proposed (Luo and Schuster, 1991; Fichtner et al., 2008; Bozdag et al., 2011; Warner and Guasch, 2016). In this thesis, we consider the crosscorrelation traveltime shift misfit functional (Luo and Schuster, 1991; Marquering et al., 1999), which quantifies the differences between predicted and measured first-arrival times. In contrast to waveform differences, the non-linearities of traveltimes with respect to the velocity perturbations are weak, and it justifies the linearized assumptions introduced in Equation (4.5) (Mercerat and Nolet, 2012).

Similar to ray-based tomography, the problem is formulated as a least-squares minimization of the traveltime differences. The key step here is to define the residuals as the time delays corresponding to the maximum of the cross-correlations between the observed and modelled signals. Adjoint techniques can then be applied to compute the Fréchet derivatives of the traveltimes with respect to the velocity. Indeed, these are the components of the Jacobian operator once the forward problem is linearized with respect to some prior information. The linearized method is often referred as finite-frequency tomography and we describe it in detail in Chapter 5 as one of the contributions of this thesis. In the following, however, we introduce the key ingredients of the method.

Assume that $T\left(\mathbf{x}_{s}{ }^{i}, \mathbf{x}_{r}{ }^{i}\right)$ is the traveltime difference between the observed and modelled signal (in $c_{0}$ ) computed from the cross-correlations. Anomalies in the traveltime difference are related to the velocity perturbations through the finite-frequency sensitivity kernel $K\left(\mathbf{x} ; \mathbf{x}_{r}{ }^{i}, \mathbf{x}_{s}{ }^{i}\right)$ as (Luo and Schuster, 1991; Korta Martiartu et al., 2020)

$$
\delta T_{i}=\int_{V} K\left(\mathbf{x} ; \mathbf{x}_{r}^{i}, \mathbf{x}_{s}^{i}\right) \delta c(\mathbf{x}) d \mathbf{x}
$$

Here, the volume $V$ represents the ROI. The sensitivity kernel is given by

$$
K\left(\mathbf{x} ; \mathbf{x}_{r}^{i}, \mathbf{x}_{s}^{i}\right)=K^{i}(\mathbf{x})=-\frac{2}{c_{0}^{3}(\mathbf{x})} \int_{0}^{t_{1}} \partial_{t} p_{0}\left(\mathbf{x}, t ; \mathbf{x}_{s}^{i}\right) \partial_{t} p_{0}^{\dagger}\left(\mathbf{x}, t_{1}-t ; \mathbf{x}_{r}^{i}\right) d t
$$

where $\left[0, t_{1}\right]$ is the time interval in which the first arrivals occur. $p_{0}\left(\mathbf{x}, t ; \mathbf{x}_{s}{ }^{i}\right)$ is the pressure field propagated through $c_{0}$ due to the emitter at $\mathbf{x}_{s}{ }^{i}$, and $p_{0}^{\dagger}\left(\mathbf{x}, t_{1}-t ; \mathbf{x}_{r}{ }^{i}\right)$ is the backpropagated pressure field from the receiver at $\mathbf{x}_{r}{ }^{i}$. The dagger symbol represents the adjoint field derived from the cross-correlations (Luo and Schuster, 1991; Tromp et al., 2005). 


\section{Image reconstruction}

Equation (4.21) describes the linearized forward problem for the traveltime waveform tomography. In the discrete form, the Jacobian matrix is computed as

$$
\mathbf{F}_{i j}^{\prime}=K^{i}\left(\mathbf{x}_{j}\right) \Delta V_{j}
$$

where $\mathbf{x}_{j}$ and $\Delta V_{j}$ are the position and the volume of the $j$-th cell, respectively. The observables and model parameters are $\delta \mathbf{d}_{i}=\delta T_{i}$ and $\delta \mathbf{m}_{j}=\delta c_{j}$, respectively.

\subsubsection{Reverse-time migration: reflectivity}

RTM has recently been introduced to USCT to image the reflectivity distribution of the breast tissue (Roy et al., 2016). While the authors formulated the method in the frequency domain, here we use its counterpart in the time domain. Unlike B-mode imaging, RTM directly solves the wave equation in (4.1), and consequently, accounts for finite-frequency effects. The standard imaging condition $I(\mathbf{x})$ is based on the cross-correlation between the wavefield $p\left(\mathbf{x}, t ; \mathbf{x}_{s}\right)$ propagated forward in time from emitters at $\mathbf{x}_{s}$, and the time-reversed measured wavefield $p^{\dagger}\left(\mathbf{x}, t_{1}-t ; \mathbf{x}_{r}\right)$ propagated from the receivers at $\mathbf{x}_{r}$. Mathematically, this can be expressed as (Claerbout, 1971)

$$
I(\mathbf{x})=\sum_{s} \int_{0}^{t_{1}} p\left(\mathbf{x}, t ; \mathbf{x}_{s}\right) p^{\dagger}\left(\mathbf{x}, t_{1}-t ; \mathbf{x}_{r}\right) d t
$$

or similarly as (Dai and Schuster, 2013)

$$
I(\mathbf{x})=\sum_{s} \int_{0}^{t_{1}} \partial_{t}^{2} p\left(\mathbf{x}, t ; \mathbf{x}_{s}\right) p^{\dagger}\left(\mathbf{x}, t_{1}-t ; \mathbf{x}_{r}\right) d t
$$

The sum over $s$ stacks the contributions from all emitters.

RTM is closely related to waveform tomography when this is stated as a least-squares minimization of the residuals between measured and modelled data. In fact, Equations (4.24) and (4.25) are linked to the sensitivity kernels of the acoustic impedance and velocity, respectively (Douma et al., 2010). To draw similarities between the B-mode and RTM techniques, in the following we will consider the scatterers as velocity perturbations (imaging principle Eq. (4.25)).

Similar to B-mode imaging, RTM is based on the Born approximation of the acoustic wave equation [Equation (A.8)]. The background velocity model $c_{0}(\mathbf{x})$ is the solution to the traveltime (waveform) tomography. Hence, the perturbed wavefield $\delta p(\mathbf{x}, t)$ related to the velocity anomaly $\delta c$ is computed as

$$
\delta p(\mathbf{x}, t)=\int_{V} \frac{I\left(\mathbf{x}^{\prime}\right)}{c_{0}^{2}\left(\mathbf{x}^{\prime}\right)} G_{0}\left(\mathbf{x}, t ; \mathbf{x}^{\prime}, 0\right) *\left[G_{0}\left(\mathbf{x}^{\prime}, t ; \mathbf{x}_{s}, 0\right) * \partial_{t}^{2} f\left(\mathbf{x}_{s}, t\right)\right] d \mathbf{x}^{\prime},
$$


where $G_{0}\left(\mathbf{x}, t ; \mathbf{x}_{s}, 0\right)$ represents Green's function of the model $c_{0}(\mathbf{x})$ due to a source at $\mathbf{x}_{s}$. The detailed derivation of this equation is discussed in Appendix A. Because of the heterogeneous background model, Green's functions are computed by numerical simulations of wave propagation, which is the main difference between RTM and B-mode [see Equations (4.26) and (4.18)]. In temporal Fourier domain, Equation (4.26) becomes

$$
\delta p(\mathbf{x}, \omega)=\int_{V} \frac{-\omega^{2} I\left(\mathbf{x}^{\prime}\right)}{c_{0}^{2}\left(\mathbf{x}^{\prime}\right)} G_{0}\left(\mathbf{x}, \omega ; \mathbf{x}^{\prime}\right) G_{0}\left(\mathbf{x}^{\prime}, \omega ; \mathbf{x}_{s}\right) f(\omega) d \mathbf{x}^{\prime}
$$

where $f(\omega)$ denotes the Fourier-transformed source function, with angular frequency $\omega$. From here, it is easier to obtain the explicit expression of the Jacobian operator, which follows

$$
\mathbf{F}_{i j}^{\prime}=\frac{-\omega^{2}}{c_{0, j}^{2}} G_{0}\left(\mathbf{x}_{r}^{i}, \omega ; \mathbf{x}_{j}\right) G_{0}\left(\mathbf{x}_{j}, \omega ; \mathbf{x}_{s}^{i}\right) f(\omega) \Delta V_{j} .
$$

The observables and model parameters are $\delta \mathbf{d}_{i}=\delta p\left(\mathbf{x}_{r}^{i}, \omega ; \mathbf{x}_{s}^{i}\right)$ and $\delta \mathbf{m}_{j}=I\left(\mathbf{x}_{j}\right)$, respectively.

\subsubsection{Sensitivities in the Jacobian operator}

Figure 4.1 illustrates the information encoded in a row of the Jacobian $\mathbf{F}^{\prime}$ for each imaging technique discussed above. By definition, each row represents how sensitive a single measurement is to changes in model parameters.

For transmission data in the infinite-frequency approximation, traveltimes are only sensitive to changes in the model parameters along the geometrical ray path [Figure 4.1(a)]. When we incorporate finite-frequency effects, sensitivities extend away from the ray paths, showing an ellipsoidal shape defined by the Fresnel zones. Figures 4.1(b) and 4.1(c) show two examples with frequencies in the range of $0.2-0.8 \mathrm{MHz}$ and $1-3 \mathrm{MHz}$, respectively. The sensitivity along the ray path is low in magnitude (Marquering et al., 1999), especially observed at lower frequencies. At high frequencies, the sensitivity kernel becomes narrower, consistent with what ray theory predicts. In Chapter 5, we discuss in more detail the features of finite-frequency sensitivity kernels.

For reflection data, Figures 4.1(d) and 4.1(e) show the sensitivities of the scattered signal to perturbations in the model parameters. These examples use the sources corresponding to the frequency ranges discussed above. The sensitivity corresponds to the time-space transformation ellipsoid multiplied by a term that accounts for spherical divergence effects [see Equation (4.19)]. The size of the sensitive area is the result of the signal duration $(0.14 \mathrm{~ms})$ and frequencies. The lack of sensitivity in the interior of the ellipsoid is due to the direct arrivals, which are muted to include only the scattered information. For lower frequencies, the zero-sensitivity area is larger due to the longer period of the direct arrivals. Finite-frequency tomography sensitivities in Figures 4.1(b) and 4.1(c) are complementary 
to the reflection sensitivities. Tomography resolves the low-wavenumber components of the medium, whereas the high wavenumbers are obtained by reflection imaging (migration) (Mora, 1989). In the context of optimal experimental design, this is an important observation since it shows that an acquisition system should be optimized to jointly retrieve transmission-reflection information from the measurements. This will be the main focus of the work presented in Chapter 7. 


\section{Straight-ray transmission}

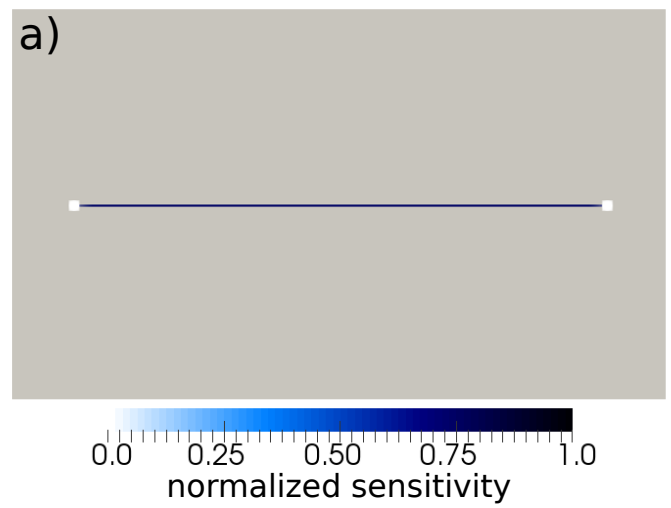

Finite-frequency transmission

b)

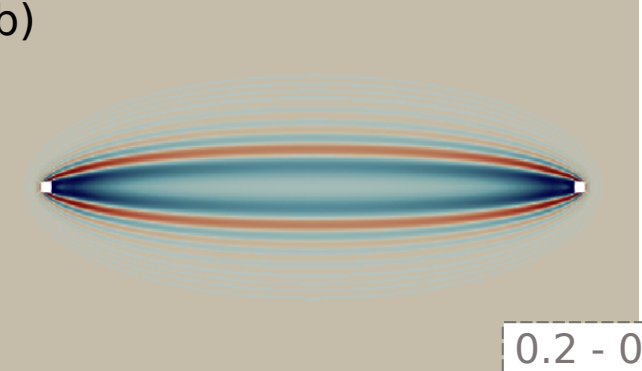

c)
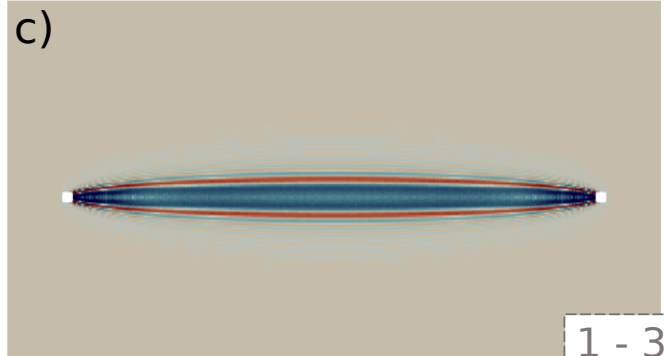

$1-3 \mathrm{MHz}$

d)

\section{e)}

Reflection
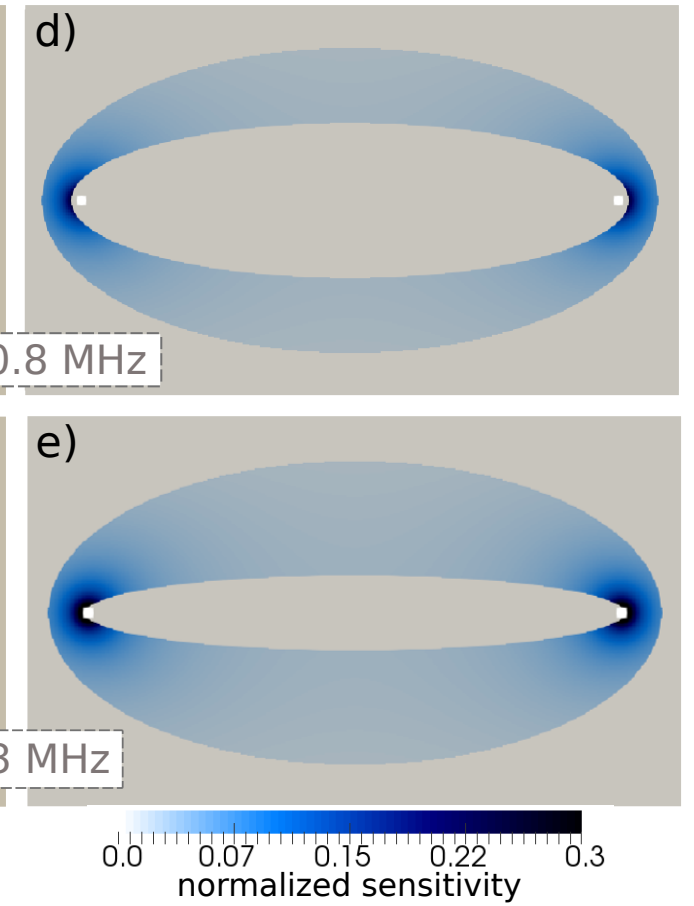

Figure 4.1.: Normalized sensitivity to model parameters encoded in the Jacobian operator of a single measurement where the distance between the emitter and receiver is $90 \mathrm{~mm}$. (a) Traveltime tomography using straight rays. (b), (c) Traveltime waveform tomography using a broad-band pulse of $0.2-0.8 \mathrm{MHz}$ and 1-3 MHz, respectively. (d), (e) Reflection imaging technique, with direct arrivals muted, corresponding to the sources in (b) and (c), respectively. The white dots indicate the emitter-receiver locations. 


\section{Image reconstruction}

\subsection{Inverse theory}

This section introduces approaches to solve linear inverse problems. Our goal is to find the model parameters $\delta \mathbf{m}$ that explain best the (noisy) measurements $\delta \mathbf{d}$, given a linear parameter-to-observable mapping defined by the forward operator $\mathbf{F}^{\prime}$. By using an illustrative introductory example, we first describe the properties of $\mathbf{F}^{\prime}$ that arise in typical USCT image reconstruction applications, which are ill-posed. To solve such inverse problems, we can use two conceptually different approaches, involving either probabilistic or deterministic optimizations. Here, we describe both inversion methods and explain the link between them that can nicely be established for linear problems. We conclude this section by providing tools for uncertainty and resolution analysis of the inferred model parameters.

\subsubsection{Properties of the forward operator}

One of the main difficulties arising in image reconstruction is related to the ill-posedness of the underlying inverse problem. This means that at least one of the following conditions is not satisfied (Hadamard, 1952):

1. Existence: For all data $\delta \mathbf{d}$, a solution $\delta \mathbf{m}$ exists.

2. Uniqueness: For all data $\delta \mathbf{d}$, the solution $\delta \mathbf{m}$ is unique.

3. Stability: The solution depends continuously on the data $\delta \mathbf{d}$, that is, small changes in $\delta \mathbf{d}$ result in small changes in $\delta \mathbf{m}$.

The ill-posedness of the inverse problem depends on the properties of the forward operator. To understand this, we use the illustrative example shown in Figure 4.2(a), where we consider straight-ray traveltime tomography for the forward modelling [see Equation (4.9)]. The velocity model is quite simple, it has a rectangular heterogeneity in a homogeneous background, and it is illuminated by emitters and receivers surrounding it. Because the forward operator does not depend on the model parameters, we do not focus our attention on the velocity structure now. This is going to be useful afterwards, to explore different regularization methods for deterministic inverse approaches in Section 4.2.3. Instead, we focus on (1) the emitter-receiver geometry, which gives a total of $14 \times 14=196$ measurements, and (2) the parameterization (discretization) of our domain of interest, a square of size $6 \mathrm{~cm}$ indicated by dashed line in Figure 4.2(a). We use for the latter a rectilinear grid of $0.5 \mathrm{~cm}$ mesh size, giving a total of $13 \times 13=169$ parameters. The forward operator can therefore be expressed by a matrix of dimensions $M \times N=196 \times 169$.

As a reminder, the forward problem was written as [Equation (4.6)]

$$
\delta \mathbf{d}=\mathbf{F}^{\prime}(\mathbf{s}) \delta \mathbf{m},
$$




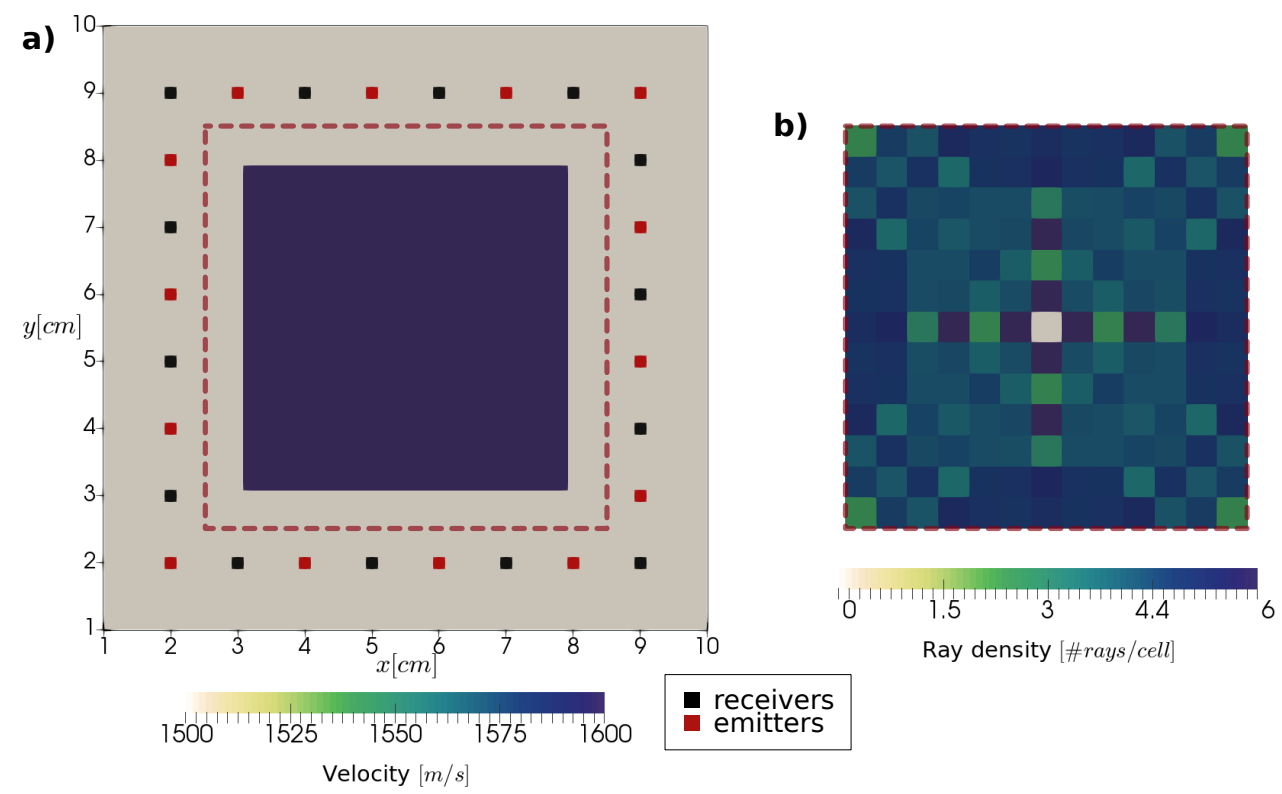

Figure 4.2.: Illustrative experiment. (a) True velocity model and the emitter-receiver configuration. Dashed line indicates the ROI. (b) Ray density indicating the number of rays crossing each location of velocity parameters.

where we have neglected the noise for this example. Here, $\mathbf{F}^{\prime}(\mathbf{s})$ is a rectangular matrix, and in principle, its inverse does not exist. The simplest way to solve this equation is by multiplying both sides by $\mathbf{F}^{\prime}(\mathbf{s})^{T}$, where the superscript $T$ represents the transpose operation of a vector or matrix. This leads to the Moore-Penrose pseudoinverse (Penrose, 1955), and the solution is estimated by

$$
\delta \mathbf{m}_{\mathrm{est}}=\left(\mathbf{F}^{\prime}(\mathbf{s})^{T} \mathbf{F}^{\prime}(\mathbf{s})\right)^{-1} \mathbf{F}^{\prime}(\mathbf{s})^{T} \delta \mathbf{d}_{\mathrm{obs}} .
$$

Note that here we are not being very strict with the notation, since we replaced the data by $\mathbf{d}_{\text {obs }}$. This will become more clear in Section 4.2.3, once deterministic approaches are introduced, but it serves now as introductory example.

From Equation (4.29) we observe that instability in the solution may appear if $\mathbf{F}^{\prime}(\mathbf{s})^{T} \mathbf{F}^{\prime}(\mathbf{s})$ is (nearly) singular or poorly conditioned, that is, if very small eigenvalues exist. We can decompose numerically the matrix $\mathbf{F}^{\prime}(\mathbf{s})^{T} \mathbf{F}^{\prime}(\mathbf{s})$ into its eigenvalues and eigenvectors, or similarly, we can perform a singular value decomposition (SVD) of $\mathbf{F}^{\prime}$ (s) (e.g., Nolet, 2008, chapter 14). By construction, both decompositions are related. Equation (4.29) shows that measurements errors in data $\delta \mathbf{d}_{\text {obs }}$ will propagate into the solution $\delta \mathbf{m}$ proportional to the inverse of the magnitude of the eigenvalues. Therefore, if small eigenvalues exist the solution becomes unstable and unreliable, the inverse problem being ill-posed. 


\section{Image reconstruction}

To understand how singularities may appear in image reconstruction applications, we use the example in Figure 4.2(a) and compute the singular value spectrum of the corresponding forward operator, shown in Figure 4.3. This is a representation of the singular values of $\mathbf{F}^{\prime}(\mathbf{s})$ in order of decreasing magnitude. The forward problem is in principle over-determined $\left(\mathbf{F}^{\prime}(\mathbf{s})\right.$ has dimensions $\left.196 \times 169\right)$. Yet, it has very small (or nearly zero) singular values between the first 169 values, corresponding to the number of unknowns. Figure 4.2(b) shows the data coverage or ray density of our problem. As we observe, singularities in the forward operator result from (1) the poor illumination of the acquisition system, which does not provide rays crossing all discrete parameters (notice the center of the ROI), and (2) redundancies in the data. These two characteristics are general for tomographic problems in USCT, especially the last one, which is a consequence of inferring properties of the media from measurements at its boundaries.

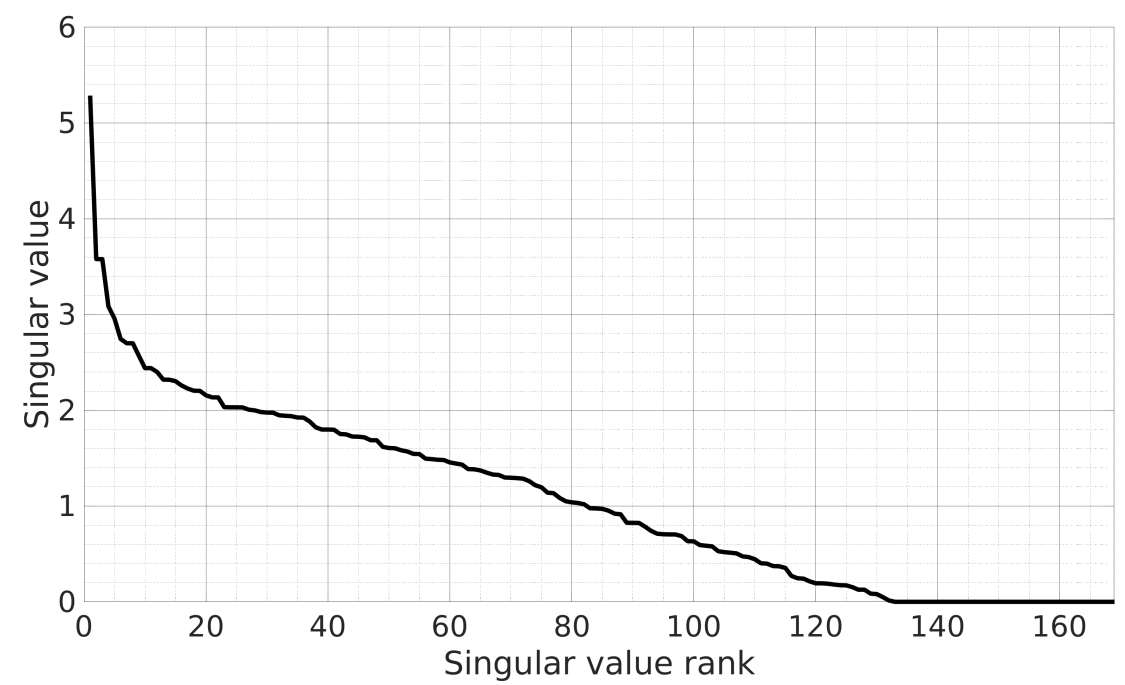

Figure 4.3.: Singular value spectrum of the forward operator $\mathbf{F}^{\prime}(\mathbf{s})$ related to the experiment in Figure 4.2 .

The singular vectors in the parameter space, which are equivalent to the eigenvectors of $\mathbf{F}^{\prime}(\mathbf{s})^{T} \mathbf{F}^{\prime}(\mathbf{s})$, provide a natural basis for discretizing the model parameters. On the one hand, eigenvectors related to non-zero eigenvalues correspond to independent combinations of the parameters that are accessible to the experiment and can be resolved (to certain extent) by the inverse operator. Eigenvectors associated to zero eigenvalues, on the other hand, show the combinations of parameters, i.e., information about the ROI, that is not recoverable from the data acquired in the experiment. The magnitude of each eigenvalue, thus, is directly related to the amount of information that the experiment can provide about specific parts of the domain. The SVD of the forward operator $\mathbf{F}^{\prime}(\mathbf{s})$ is therefore 
useful to understand which parts of the domain can be resolved by a given acquisition system, and analyzing this will be the main focus of optimal experimental design methods, studied in Chapters 6 and 7.
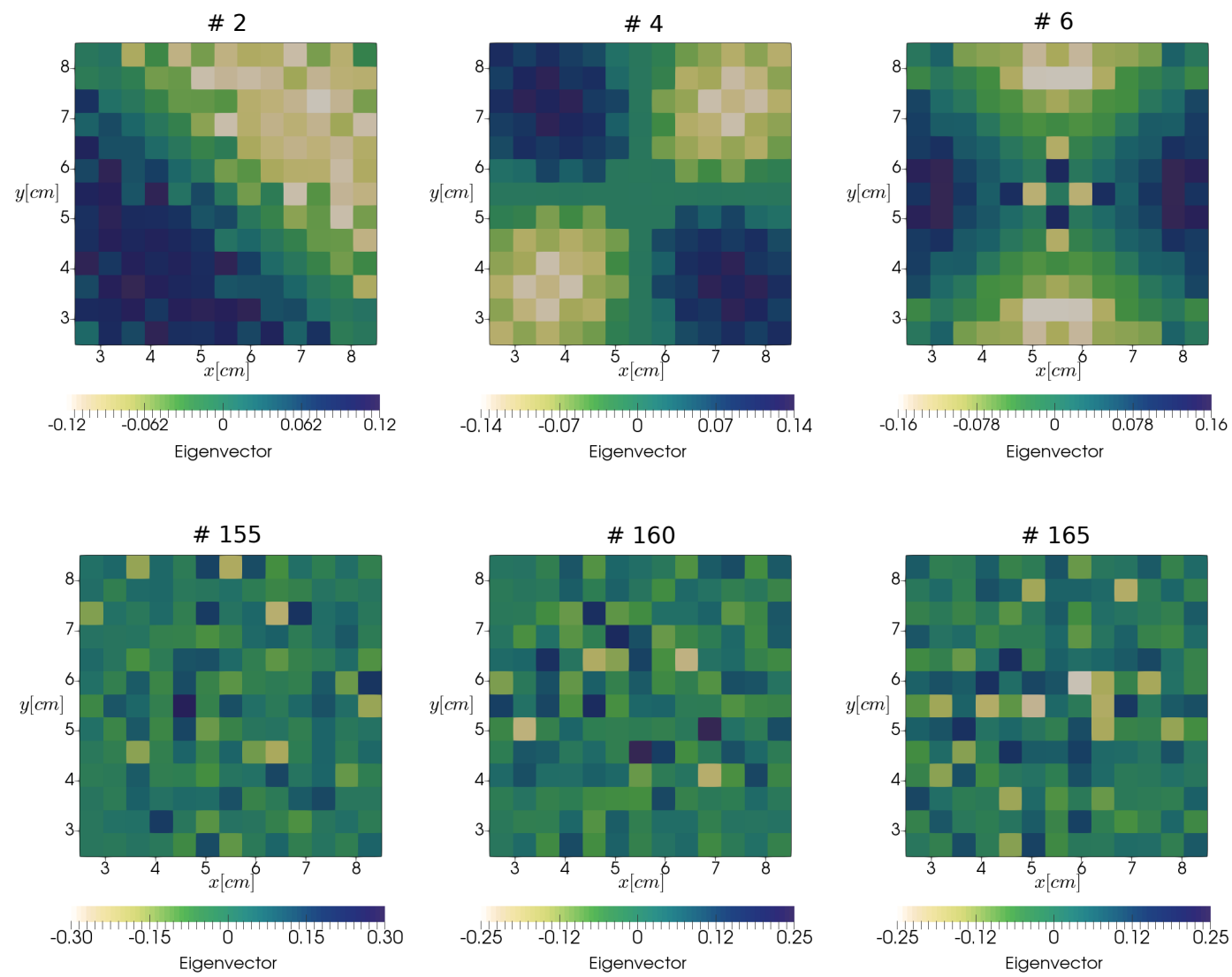

Figure 4.4.: Eigenvectors of $\mathbf{F}^{\prime}(\mathbf{s})$, or $\mathbf{F}^{\prime}(\mathbf{s})^{T} \mathbf{F}^{\prime}(\mathbf{s})$, related to the experiment in Figure 4.2. In each case, the number indicates the rank of the corresponding eigenvalue.

The singular value spectrum in Figure 4.3 shows a rapid decay in magnitude, which is common for image reconstruction problems. Dominant eigenvalues are in general informative about eigenvectors that are smooth. Examples of these are visualized in Figure 4.4 (top row), which reveal that the data mainly sees the medium in average terms, and often, local features cannot be resolved. This information appears in eigenvectors related to the smallest singular values [see bottom row of Figure 4.4], which are more oscillatory. Due to the rapid decay of the spectrum, the data are mainly informative about a low dimensional subspace of the model parameters. The most significant singular values determine the effective rank of the operator $\mathbf{F}^{\prime}(\mathbf{s})$, or equivalently $\mathbf{F}^{\prime}(\mathbf{s})^{T} \mathbf{F}^{\prime}(\mathbf{s})$, and this can be useful to 


\section{Image reconstruction}

reduce the dimensionality of the problem. The rest of the singular values can be considered as defining the effective model nullspace. Here, different combinations of model parameters lying onto the eigenvectors of the nullspace can predict very similar observables (Deal and Nolet, 1996), and therefore, the solution becomes non-unique.

In the following, we address the ill-posed nature of the inverse problems from different perspectives. These approaches incorporate some prior information about the model parameters into the formulation of inverse problems to make them well-posed.

\subsubsection{Bayesian inverse problem}

Measurement noise, limited data coverage, and the approximations in the forward operator introduce uncertainties into the estimated parameters, and statistical approaches can be adopted to solve the inverse problem. From Bayesian inference theory, the solution to the inverse problem is described as a posterior probability density function $\pi(\mathbf{m} \mid \mathbf{d} ; \mathbf{s})$ (e.g., Tarantola, 2005), which combines the data likelihood $\pi(\mathbf{d} \mid \mathbf{m} ; \mathbf{s})$ and our prior beliefs about model parameters $\pi(\mathbf{m})$,

$$
\pi(\mathbf{m} \mid \mathbf{d} ; \mathbf{s}) \propto \pi(\mathbf{d} \mid \mathbf{m} ; \mathbf{s}) \pi(\mathbf{m}) .
$$

Here, the missing normalization term is the marginal probability density of the data, named the evidence and often ignored because it only scales the posterior probability $\pi(\mathbf{m} \mid \mathbf{d} ; \mathbf{s})$.

The data likelihood explicitly depends on $\mathbf{d}_{\mathrm{obs}}$ and represents the degree of similarity between predicted and observed data, while taking into account the noise in the measurements. As mentioned in Equation (4.4), we assume this to be Gaussian with zero-mean

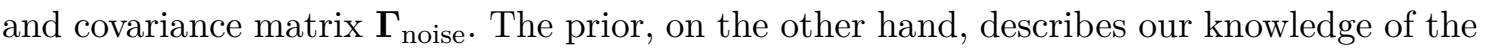
model parameters that is independent of the data, and for simplicity, we also assume to be Gaussian, with mean $\mathbf{m}_{\text {prior }}$ and covariance matrix $\boldsymbol{\Gamma}_{\text {prior }}$. In such situations, and for linear forward problems, the posterior $\pi(\mathbf{m} \mid \mathbf{d} ; \mathbf{s}$ ) becomes Gaussian (Tarantola, 2005), and it is expressed as

$$
\pi(\mathbf{m} \mid \mathbf{d} ; \mathbf{s}) \propto \exp \left[-\frac{1}{2}\left(\left\|\delta \mathbf{d}_{\mathrm{obs}}-\mathbf{F}^{\prime}(\mathbf{s}) \delta \mathbf{m}\right\|_{\Gamma_{\text {noise }}^{-1}}^{2}+\|\delta \mathbf{m}\|_{\boldsymbol{\Gamma}_{\text {prior }}^{-1}}^{2}\right)\right] .
$$

Here, the weighted norms are defined as $\|\mathbf{x}\|_{\boldsymbol{\Gamma}^{-1}}^{2}=\mathbf{x}^{T} \boldsymbol{\Gamma}^{-1} \mathbf{x}$ and $\delta \mathbf{m}=\mathbf{m}-\mathbf{m}_{\text {prior }}$. By rearranging the terms, we find that

$$
\pi(\mathbf{m} \mid \mathbf{d} ; \mathbf{s}) \propto \exp \left[-\frac{1}{2}(\delta \mathbf{m}-\widetilde{\delta \mathbf{m}})^{T} \boldsymbol{\Gamma}_{\text {post }}^{-1}(\delta \mathbf{m}-\widetilde{\delta \mathbf{m}})\right]
$$

with mean

$$
\widetilde{\delta \mathbf{m}}=\boldsymbol{\Gamma}_{\text {post }}(\mathbf{s}) \mathbf{F}^{\prime}(\mathbf{s})^{T} \boldsymbol{\Gamma}_{\text {noise }}^{-1} \delta \mathbf{d}_{\mathrm{obs}},
$$


and posterior covariance matrix

$$
\boldsymbol{\Gamma}_{\text {post }}(\mathbf{s})=\left(\mathbf{F}^{\prime}(\mathbf{s})^{T} \boldsymbol{\Gamma}_{\text {noise }}^{-1} \mathbf{F}^{\prime}(\mathbf{s})+\boldsymbol{\Gamma}_{\text {prior }}^{-1}\right)^{-1}
$$

Because the posterior is Gaussian, the mean is the maximum a posteriori point satisfying

$$
\widetilde{\delta \mathbf{m}}=\underset{\mathbf{m}}{\arg \max } \pi(\mathbf{m} \mid \mathbf{d} ; \mathbf{s}),
$$

with uncertainties encoded in the posterior covariance matrix $\boldsymbol{\Gamma}_{\text {post }}(\mathbf{s})$. Note that in probabilistic approaches the solution to the inverse problem is given by the complete marginal probability density in the model space, i.e., by the posterior $\pi(\mathbf{m} \mid \mathbf{d} ; \mathbf{s})$, and not by its mean $\widetilde{\delta \mathbf{m}}$. The former contains all plausible models ranked by their probability.

The Bayesian inference theory is a very elegant and flexible tool, and it does not put constraints on the nature of the forward problem, measurement noise and prior. However, linearized forward problems are often used in order to obtain analytical expressions of the posterior and, thus, solutions that are computationally tractable. These may introduce modelling uncertainties that we could account for using the same Bayesian framework. We can modify the data likelihood to incorporate modelling errors. For instance, by assuming, for simplicity, Gaussian modelling errors with covariance $\boldsymbol{\Gamma}_{\mathrm{T}}$, the data likelihood becomes

$$
\pi(\mathbf{m} \mid \mathbf{d} ; \mathbf{s}) \propto \exp \left[-\frac{1}{2}\left(\left\|\delta \mathbf{d}_{\mathrm{obs}}-\mathbf{F}^{\prime}(\mathbf{s}) \delta \mathbf{m}\right\|_{\Gamma_{\text {noise }}^{-1}}^{2}+\left\|\delta \mathbf{d}_{\mathrm{obs}}-\mathbf{F}^{\prime}(\mathbf{s}) \delta \mathbf{m}\right\|_{\Gamma_{\mathrm{T}}^{-1}}^{2}\right)\right],
$$

which is equivalent to replacing the noise covariance with $\boldsymbol{\Gamma}_{\text {noise }}^{-1}+\boldsymbol{\Gamma}_{\mathrm{T}}^{-1}$ in the derivations above. While Gaussian assumptions allow us to account for modelling errors in a very straightforward manner, in reality these are more complex and difficult to quantify. This is why we usually omit them from the inversion approach.

\subsubsection{Deterministic inversion}

In USCT, deterministic approaches to solve inverse problems are more popular, and often the solution is formulated as a least-squares minimization problem. Contrary to probabilistic approaches, here the solution is given by the model that best explains our observations. For linear problems, with Gaussian noise and prior, however, we can establish the link between both inference perspectives.

From Equation (4.35) we see that finding $\widetilde{\delta \mathbf{m}}$ is equivalent to solving the weighted least-squares optimization problem

$$
\widetilde{\delta \mathbf{m}}=\underset{\mathbf{m}}{\arg \min }\left\{\chi(\delta \mathbf{m}):=\frac{1}{2}\left(\left\|\delta \mathbf{d}_{\text {obs }}-\mathbf{F}^{\prime}(\mathbf{s}) \delta \mathbf{m}\right\|_{\Gamma_{\text {noise }}^{-1}}^{2}+\|\delta \mathbf{m}\|_{\boldsymbol{\Gamma}_{\text {prior }}^{-1}}^{2}\right)\right\} .
$$

The model parameters are therefore estimated by minimizing an objective functional $\chi(\delta \mathbf{m})$ 


\section{Image reconstruction}

that is the combination of two terms, the data-misfit and regularization term, respectively. Here, $\boldsymbol{\Gamma}_{\text {prior }}^{-1}$ and $\boldsymbol{\Gamma}_{\text {noise }}^{-1}$ weight the contribution of each term in the objective functional. In this approach, the posterior covariance matrix $\boldsymbol{\Gamma}_{\text {post }}(\mathbf{s})$ corresponds to the inverse of the Hessian, i.e., the second derivative of $\chi(\delta \mathbf{m})$ with respect to $\delta \mathbf{m}$,

$$
\mathbf{H}(\mathbf{s})=\chi^{\prime \prime}(\delta \mathbf{m})=\boldsymbol{\Gamma}_{\text {post }}^{-1}(\mathbf{s})=\mathbf{F}^{\prime}(\mathbf{s})^{T} \boldsymbol{\Gamma}_{\text {noise }}^{-1} \mathbf{F}^{\prime}(\mathbf{s})+\boldsymbol{\Gamma}_{\text {prior }}^{-1} .
$$

The first term is the data-misfit Hessian,

$$
\mathbf{H}_{\text {misfit }}(\mathbf{s})=\mathbf{F}^{\prime}(\mathbf{s})^{T} \boldsymbol{\Gamma}_{\text {noise }}^{-1} \mathbf{F}^{\prime}(\mathbf{s}),
$$

and as we will observe in Chapters 6 and 7, it has a fundamental role in optimal experimental design methods.

Now, let us generalize the notation in Equation (4.37) to extend to non-Gaussian priors. The objective functional can be rewritten as

$$
\chi(\delta \mathbf{m}):=\frac{1}{2}\left\|\delta \mathbf{d}_{\mathrm{obs}}-\mathbf{F}^{\prime}(\mathbf{s}) \delta \mathbf{m}\right\|_{\Gamma_{\text {noise }}^{-1}}^{2}+\alpha J(\delta \mathbf{m}),
$$

where $J(\delta \mathbf{m})$ is the regularization term, and $\alpha>0$ is the regularization parameter that balances the contribution of both terms. Unless Gaussian priors are used, the solution that minimizes $\chi(\delta \mathbf{m})$ cannot be expressed analytically, and the regularized least-squares problem can be efficiently solved using an iterative method such as LSQR (Paige and Saunders, 1982a). These algorithms have the potential to solve large systems of equations without explicitly constructing the forward matrix $\mathbf{F}^{\prime}(\mathbf{s})$, which is essential for the studies considered in this thesis.

The regularization term incorporates our prior information about the model parameters to mitigate the ill-posedness of the inverse problem and to ensure meaningful solutions. The choice of the regularization method depends on the specific application under consideration, and it allows us to reduce a set of equally satisfactory solutions by using knowledge based on empirical observations. In the following, we describe the properties of different regularization approaches that are commonly applied in USCT and were implemented during this thesis.

\subsubsection{Regularization approaches}

This subsection uses the illustrative example in Figure 4.2 to visualize the effects of different regularization approaches. To make the ill-posedness more noticeable, we refine the mesh in which the model is discretized using a rectilinear grid of $0.1 \mathrm{~cm}$ mesh size. 


\subsubsection{Tikhonov regularization}

Tikhonov regularization methods are described by a family of functions that follows

$$
J(\delta \mathbf{m}):=\|\mathbf{L} \delta \mathbf{m}\|^{2},
$$

where $\mathbf{L}$ is typically a linear operator that equals the identity matrix or approximates discrete derivatives of arbitrary order (Tikhonov, 1963), $\mathbf{L}^{T} \mathbf{L}$ being positive definite. In comparison to Equation (4.32), we observe that Tikhonov methods include Gaussian priors and, therefore, offer analytical expressions of the regularized solutions satisfying

$$
\delta \mathbf{m}_{\text {est }}=\left(\mathbf{F}^{\prime}(\mathbf{s})^{T} \boldsymbol{\Gamma}_{\text {noise }}^{-1} \mathbf{F}^{\prime}(\mathbf{s})+\alpha \mathbf{L}^{T} \mathbf{L}\right)^{-1} \mathbf{F}^{\prime}(\mathbf{s})^{T} \boldsymbol{\Gamma}_{\text {noise }}^{-1} \delta \mathbf{d}_{\text {obs }} .
$$

Here, we have changed the notation to $\delta \mathbf{m}_{\text {est }}$ to highlight the difference with respect to probabilistic approaches.

1. Zeroth-order Tikhonov: In USCT applications, we often have preference for reconstructions which are close to some reference model $\mathbf{m}_{\text {prior }}$. As mentioned before, we usually know that the breast tissue shows relatively small variations, and $\mathbf{m}_{\text {prior }}$ can for instance refer to the medium in which calibration data are recorded. In such cases, $\mathbf{L}=\mathbf{I}$, and the regularization term ensures minimal $L_{2}$-distance with respect to $\mathbf{m}_{\text {prior }}$. An example of reconstruction using zeroth-order Tikhonov is shown in Figure 4.5 (left image). Here, the reference model is a homogeneous medium with velocity $1500 \mathrm{~m} / \mathrm{s}$. As we observe, the effects are generally strongest in regions with poor data coverage, and in the extreme case of not being illuminated at all, the solution takes the values corresponding to $\mathbf{m}_{\text {prior }}$.

2. First- and second-order Tikhonov: Usually, the structure of the medium varies smoothly, and one may wish to include an operator $\mathbf{L}$ that gives preference to solutions with smooth variations. A common strategy is to replace $\mathbf{L}$ by a finite-difference operator acting on the model space. This often includes either first- or second-order derivatives. Assuming 1D model for simplicity, the operator $\mathbf{L}$ that approximates first-order derivatives can be expressed as

$$
\mathbf{L}=\left(\begin{array}{cccc}
-1 & 1 & & \\
& -1 & 1 & \\
& & \ddots & \ddots
\end{array}\right)
$$




\section{Image reconstruction}

whereas for the second-order derivatives is

$$
\mathbf{L}=\left(\begin{array}{ccccc}
-1 & 2 & -1 & & \\
& -1 & 2 & -1 & \\
& & \ddots & \ddots & \ddots
\end{array}\right)
$$

which is the Laplacian operator.

Non-smooth or oscillatory solutions will lead to large values of the derivatives, and these are penalized by the regularization term. An example of reconstruction using second-order Tikhonov is shown in Figure 4.5 (middle image). The solution shows smooth features, and for this particular example, the regularization blurs the edges of the heterogeneity, in which the true velocity contrasts are sharp.
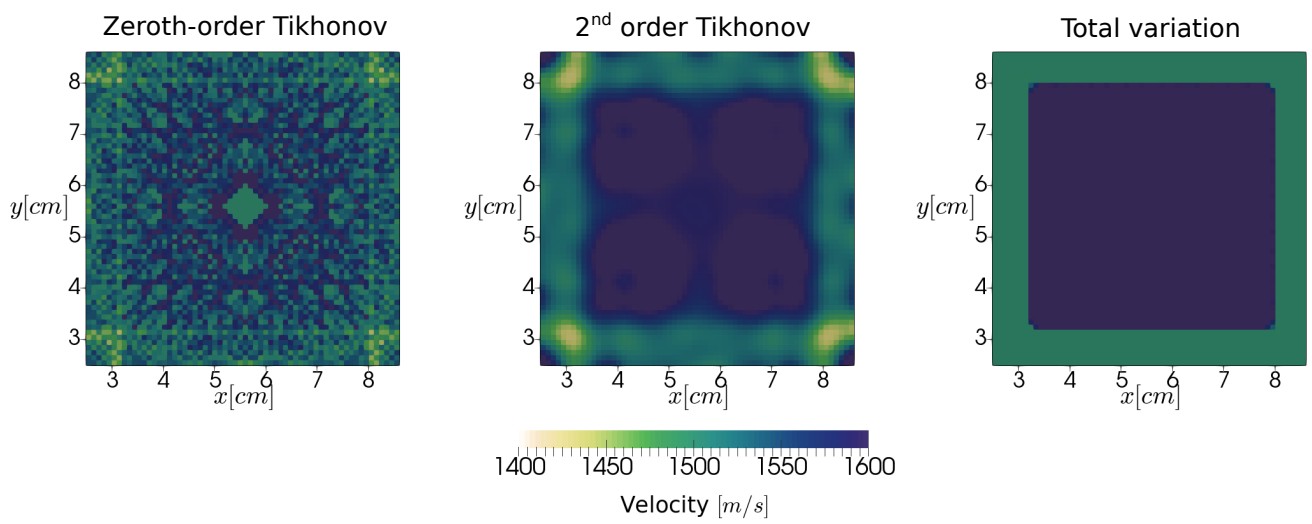

Figure 4.5.: Velocity reconstruction for the illustrative example in Figure 4.2. (From left to right) We apply zeroth- and first-order Tikhonov and TV regularization, respectively. This numerical example does not consider measurement noise of any type, and thus, we allow inverse crime. For a fair comparison of the results, we find solutions with similar data residuals.

To understand the effect of Tikhonov regularization in filtering small singular values of the forward operator $\mathbf{F}^{\prime}(\mathbf{s})$, we assume $\mathbf{L}=\mathbf{I}$ and $\boldsymbol{\Gamma}_{\text {noise }}=\mathbf{I}$ for simplicity. By using SVD, we can express $\mathbf{F}^{\prime}(\mathbf{s})$ as

$$
\mathbf{F}^{\prime}=\mathbf{U S V}^{\mathbf{T}}
$$

where $\mathbf{S} \in \mathbf{R}^{M \times N}$ is a diagonal matrix with singular values $\lambda_{i}$ as its elements, and $\mathbf{U} \in$ $\mathbf{R}^{M \times M}$ and $\mathbf{V} \in \mathbf{R}^{N \times N}$ are orthogonal matrices composed by singular vectors in data space $\mathbf{u}_{i}$ and model space $\mathbf{v}_{i}$, respectively. Upon inserting Equation (4.45) into Equation (4.42), 
we obtain

$$
\delta \mathbf{m}_{\mathrm{est}}=\sum_{i=1}^{\min \{M, N\}} \frac{\lambda_{i}}{\lambda_{i}^{2}+\alpha}\left(\mathbf{u}_{i} \cdot \delta \mathbf{d}_{\mathrm{obs}}\right) \mathbf{v}_{i} .
$$

The term $\frac{\lambda_{i}}{\lambda_{i}^{2}+\alpha}$ weights the contribution of different singular vectors in the representation of the solution. We therefore observe that Tikhonov regularization filters small singular values through the regularization parameter $\alpha$. Figure 4.6 shows a comparison between the eigenvalue spectra of $\mathbf{F}^{\prime}(\mathbf{s})^{T} \mathbf{F}^{\prime}(\mathbf{s})$ and $\mathbf{F}^{\prime}(\mathbf{s})^{T} \mathbf{F}^{\prime}(\mathbf{s})+\alpha \mathbf{I}$, where $\alpha=5 \cdot 10^{-3} \lambda_{1}$. Here, we also include the spectra corresponding to regularizations using higher-order Tikhonov approaches. In general, the optimal regularization parameter varies for different approaches, and the Figure 4.6 may not be a fair comparison. However, it serves as illustrative example to understand the effects of the regularization term in the spectrum of the forward operator. Whereas the zeroth-order Tikhonov shifts all the spectrum by the same quantity, the firstand second-order Tikhonov affects each part of the spectrum in different degree, the lower part being the most varying one.

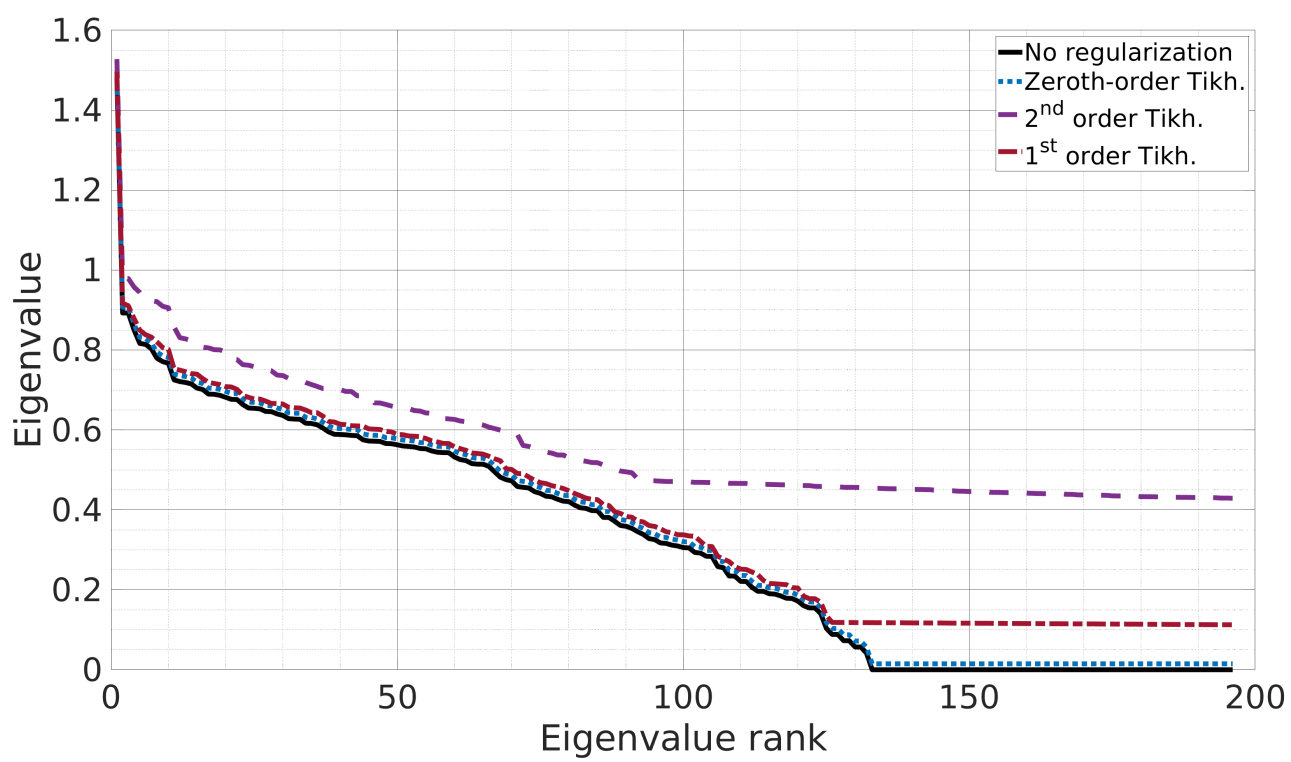

Figure 4.6.: Comparison between the eigenvalue spectra of the data-misfit and regularized Hessian. It uses the illustrative example in Figure 4.2, with a rectilinear grid of $0.1 \mathrm{~cm}$ mesh size. With such discretization of the parameters, the problem becomes highly under-determined, and we plot the first 196 eigenvalues (same as the number of measurements). We use zeroth-, first- and second-order Tikhonov regularizations, with the regularization parameter $\alpha=5 \cdot 10^{-3} \lambda_{1}$. 


\section{Image reconstruction}

\subsubsection{Total variation regularization}

In image reconstruction, we are often interested in imaging sharp interfaces between different tissue structures. Tikhonov regularization, although being very popular, blurs these interfaces, and other alternatives are often preferred. Total variation (TV) regularization is a commonly used edge-preserving technique (e.g Matthews et al., 2017; Li et al., 2009). Here, the regularization term is defined as

$$
J(\delta \mathbf{m}):=\int_{V}\|\nabla \delta \mathbf{m}\| d \mathbf{x} .
$$

TV regularization includes the integration over our domain of interest $V$, and therefore, it minimizes the cumulative deviation from zero of the derivatives. Consequently, it becomes suitable to reconstruct piecewise constant solutions, allowing the presence of sharp interfaces. Figure 4.5 (right image) shows an example of velocity reconstruction using TV regularization. In this particular example, the true model is piecewise constant, and thus, the performance of TV regularization is significantly superior to other approaches.

When using the regularization term in Equation (4.47), we drop the assumption of Gaussian priors, and thus, analytical expressions of the regularized solutions are not available. The work presented in this thesis mostly applies TV regularization and uses the software implemented by Jensen et al. (2012), which is publicly available.

\subsubsection{Truncated SVD}

The truncation of the singular value spectrum of the forward operator can also be seen as a regularization technique that aims at mitigating the ill-posedness of the inverse problem. We can understand it as a filtering approach, very similar to the Tikhonov regularization described in Equation (4.46). By retaining the singular values satisfying $\lambda_{i}^{2}>\alpha$, the solution can be expressed as

$$
\delta \mathbf{m}_{\mathrm{est}}=\sum_{\lambda_{i}^{2}>\alpha}^{\min \{M, N\}} \frac{1}{\lambda_{i}}\left(\mathbf{u}_{i} \cdot \delta \mathbf{d}_{\mathrm{obs}}\right) \mathbf{v}_{i}
$$

From here, we see that, unless the condition $\lambda_{i}^{2}>\alpha$ is imposed, instabilities in the solution may arise from small singular values, while measurement errors being amplified proportional to $\frac{1}{\lambda_{i}}$.

The truncation of the spectrum is useful to compute low-rank approximations of large matrices and their inverse. This is indeed the underlying idea in Equation (4.48), and it could be used for multiple purposes. In image reconstruction problems, we have seen that the eigenvalue spectrum of $\mathbf{F}^{\prime}(\mathbf{s})^{T} \mathbf{F}^{\prime}(\mathbf{s})$ decays very rapidly in magnitude [see Figure 4.4]. We can exploit this structure to efficiently compute the inverse of large matrices by retain- 
ing very few eigenvalues and their corresponding eigenvectors. As mentioned before, the eigenvectors associated with dominant eigenvalues are smooth, and refinements of the mesh will not affect these eigenvalues (at least, once a suitable discretization is obtained) (Martin et al., 2012). Thus, the effective rank is independent of the number of parameters, and consequently, it allows us to efficiently reduce the dimensionality of large-scale problems. This invariant character can be noticed from the comparison of Figures 4.3 and 4.6. In particular, low-rank approximations are key for uncertainty and resolution analysis of large-scale problems.

\subsubsection{Uncertainty and resolution analysis}

The quantification of resolution and uncertainties is an essential part of inverse problems, and it allows us to provide a comprehensive description of the solutions. In linear inverse problems, with Gaussian noise and prior, these can be expressed analytically. For instance, uncertainties in the solution are described by means of the posterior covariance

$$
\boldsymbol{\Gamma}_{\text {post }}=\left(\mathbf{F}^{\prime T} \boldsymbol{\Gamma}_{\text {noise }}^{-1} \mathbf{F}^{\prime}+\boldsymbol{\Gamma}_{\text {prior }}^{-1}\right)^{-1},
$$

which is useful to interpret the reliability of our parameter estimations. Here, we omitted the dependency on $\mathbf{s}$ for clarity. The diagonal entries of $\boldsymbol{\Gamma}_{\text {post }}$ indicate the variances of the individual parameters, and the off-diagonal entries show the correlations between the errors in different model parameters. These may refer to either parameters at different locations or parameters describing different properties of the tissue (for multi-parameter inversions). Figure 4.7 (top row) displays the diagonal elements of the posterior covariance matrix related to the regularized solution using second-order Tikhonov [see Figure 4.5]. We find the larger variances at the edges and center of the ROI, which correlates with the data coverage.

Another useful quantity is the resolution matrix, which satisfies

$$
\delta \mathbf{m}_{\text {est }}=\mathbf{R} \delta \mathbf{m}_{\text {true }}
$$

and indicates how well the model parameters are resolved in the inversion. This is a dimensionless quantity. If we assume that the observed data fully satisfy our description of the forward modelling, the resolution matrix $\mathbf{R}$ can be analytically computed as

$$
\mathbf{R}=\left(\mathbf{F}^{T} \boldsymbol{\Gamma}_{\text {noise }}^{-1} \mathbf{F}+\boldsymbol{\Gamma}_{\text {prior }}^{-1}\right)^{-1} \mathbf{F}^{T} \boldsymbol{\Gamma}_{\text {noise }}^{-1} \mathbf{F} .
$$

For a comprehensive analysis and derivations of Equations (4.49) and (4.51) the reader is referred to Tarantola (2005). When the model parameters are perfectly resolved, $\mathbf{R}$ equals the identity matrix. In general, however, $\mathbf{R} \neq \mathbf{I}$, and the estimated parameters are weighted averages of the true parameters. Each column of $\mathbf{R}$ is interpreted as a point- 


\section{Image reconstruction}

spread function (PSF), which illustrates the blurring of one parameter into others. PSFs are therefore useful to describe how independently an individual parameter can be resolved by the data. Figure 4.7 (bottom row) shows three examples of PSFs computed for our illustrative example. These are located at positions $(x, y)=(5.5,5.5) ;(5.5,6) ;(5.5,6.5) \mathrm{cm}$. As a result of uneven data coverage, the size of PSFs, and thus, the spatial resolution, is position dependent. Due to the lack of illumination at the center of the ROI, we cannot resolve the parameter here, and the corresponding PSF is zero everywhere. The rest of the PSFs show elongated shapes following the ray paths and indicating strong inter-parameter trade-offs caused by limited data coverage.
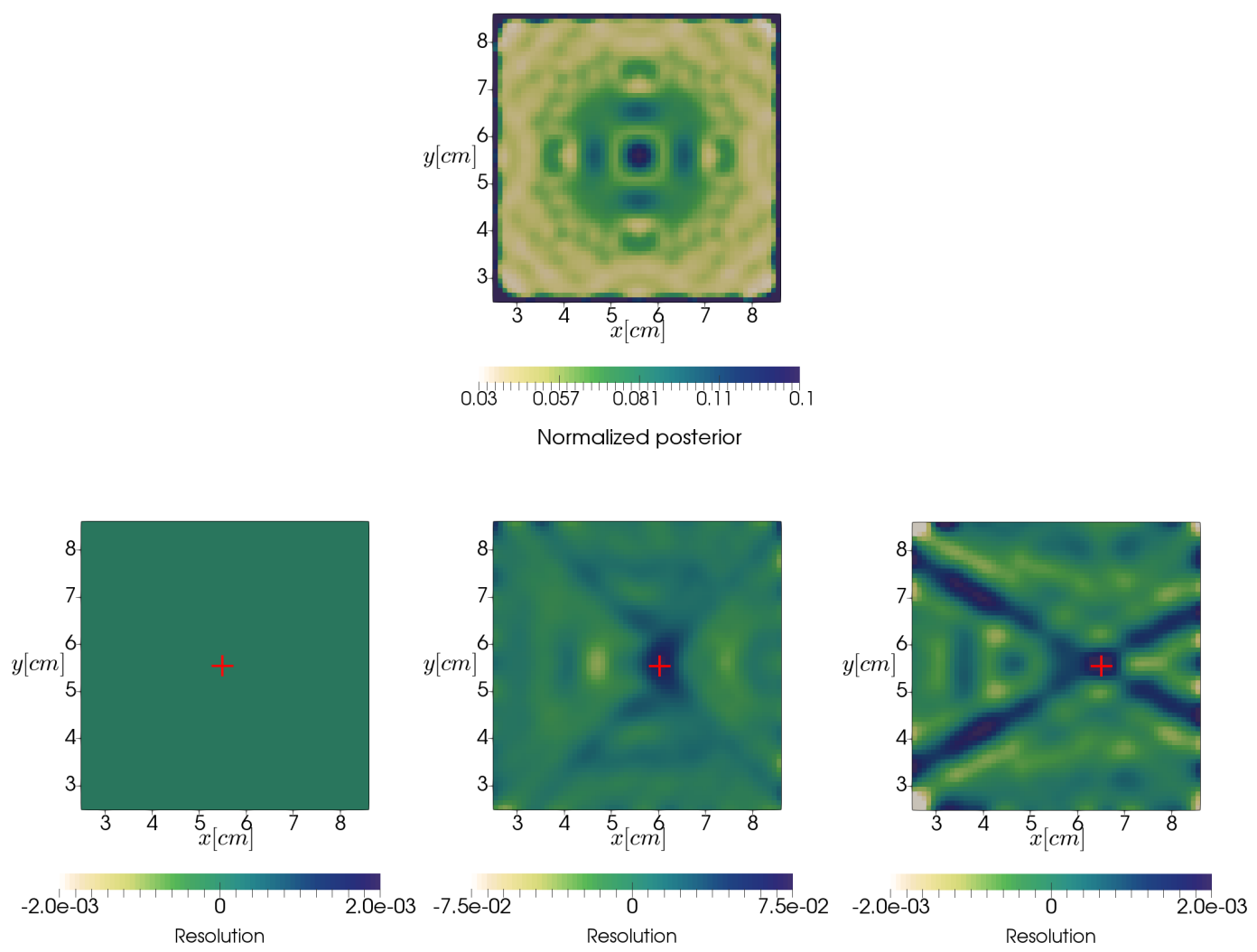

Figure 4.7.: (Top row) Normalized diagonal elements of the posterior covariance matrix for the solution in Figure 4.5 using second-order Tikhonov. (Bottom row) PSFs computed from the resolution matrix at positions $(x, y)=(5.5,5.5) ;(5.5,6) ;(5.5,6.5) \mathrm{cm}$, which is indicated in each case by the red cross.

For large-scale problems, even when $\mathbf{F}^{\prime}$ is explicitly available, computing $\boldsymbol{\Gamma}_{\text {post }}$ and $\mathbf{R}$ may be challenging. However, we can benefit from the rapidly decaying eigenvalues of $\mathbf{H}_{\text {misfit }}=\mathbf{F}^{\prime T} \boldsymbol{\Gamma}_{\text {noise }}^{-1} \mathbf{F}^{\prime}$ to compute their low-rank approximations. As we observed in 
Figure 4.6, the spectrum is affected by the prior and, instead, we will focus on the priorpreconditioned data-misfit Hessian (Flath et al., 2011). This can be identified by rearranging the terms in the expression of $\boldsymbol{\Gamma}_{\text {post }}$ as

$$
\boldsymbol{\Gamma}_{\text {post }}=\left(\mathbf{F}^{\prime T} \boldsymbol{\Gamma}_{\text {noise }}^{-1} \mathbf{F}^{\prime}+\boldsymbol{\Gamma}_{\text {prior }}^{-1}\right)^{-1}=\boldsymbol{\Gamma}_{\text {prior }}^{1 / 2}\left(\boldsymbol{\Gamma}_{\text {prior }}^{1 / 2} \mathbf{F}^{\prime T} \boldsymbol{\Gamma}_{\text {noise }}^{-1} \mathbf{F}^{\prime} \boldsymbol{\Gamma}_{\text {prior }}^{1 / 2}+\mathbf{I}\right)^{-1} \boldsymbol{\Gamma}_{\text {prior }}^{1 / 2},
$$

where the first term is the prior-preconditioned data-misfit Hessian

$$
\widetilde{\mathbf{H}}_{\text {misfit }}:=\boldsymbol{\Gamma}_{\text {prior }}^{1 / 2} \mathbf{F}^{\prime T} \boldsymbol{\Gamma}_{\text {noise }}^{-1} \mathbf{F}^{\prime} \boldsymbol{\Gamma}_{\text {prior }}^{1 / 2}
$$

We compute the low-rank approximation of $\widetilde{\mathbf{H}}_{\text {misfit }}$ by retaining the $k<N$ largest eigenvalues and the corresponding eigenvectors, that is,

$$
\widetilde{\mathbf{H}}_{\text {misfit }}=\mathbf{V} \boldsymbol{\Lambda} \mathbf{V}^{T} \approx \mathbf{V}_{k} \boldsymbol{\Lambda}_{k} \mathbf{V}_{k}^{T},
$$

where $\boldsymbol{\Lambda}=\operatorname{diag}\left(\lambda_{i}\right) \in \mathbb{R}^{N \times N}$ and $\mathbf{V} \in \mathbb{R}^{N \times N}$ are the eigenvalue and eigenvector matrix, respectively. Here, $\boldsymbol{\Lambda}_{k} \in \mathbb{R}^{k \times k}$ and $\mathbf{V}_{k} \in \mathbb{R}^{N \times k}$ represent the truncated eigenvalue and eigenvector matrices. Then, the low-rank approximations of $\boldsymbol{\Gamma}_{\text {post }}$ and $\mathbf{R}$ are given by

$$
\begin{gathered}
\boldsymbol{\Gamma}_{\text {post }} \approx \boldsymbol{\Gamma}_{\text {prior }}^{1 / 2}\left(\mathbf{I}-\mathbf{V}_{k} \mathbf{D}_{k} \mathbf{V}_{k}^{T}\right) \boldsymbol{\Gamma}_{\text {prior }}^{1 / 2}, \\
\mathbf{R} \approx \boldsymbol{\Gamma}_{\text {prior }}^{1 / 2} \mathbf{V}_{k} \mathbf{D}_{k} \mathbf{V}_{k}^{T} \boldsymbol{\Gamma}_{\text {prior }}^{-1 / 2}
\end{gathered}
$$

with $\mathbf{D}_{k}=\operatorname{diag}\left(\frac{\lambda_{i}}{\lambda_{i}+1}\right) \in \mathbb{R}^{k \times k}$. In Appendix B, we describe the derivations leading to the expressions above and we follow Flath et al. (2011).

As observed in Equation (4.55), $\boldsymbol{\Gamma}_{\text {post }}$ is the result of extracting from our prior uncertainties the information gained from the data. From Equation (4.56) we moreover observe that, when $\boldsymbol{\Gamma}_{\text {prior }}=\alpha^{-1} \mathbf{I}$ and $\lambda_{i} \gg 1$, the resolution matrix becomes

$$
\mathbf{R} \approx \mathbf{V}_{k} \mathbf{V}_{k}^{T}
$$

which has an intuitive meaning: it filters out the features of the model that lie in the effective nullspace of $\mathbf{F}^{\prime}$. For instance, we can apply this to the estimated solution to remove the artifacts introduced by the regularization term.

Whereas the derivations above assume Gaussian priors, they may still provide useful information about resolution and uncertainties when non-Gaussian priors (e.g., total variation regularization) are used for reconstructions. The regularization term mostly acts in the effective nullspace of the data-misfit Hessian, and this is removed by applying low-rank approximations.

For high-dimensional problems, the low-rank approximation of $\mathbf{R}$ may still be pro- 


\section{Image reconstruction}

hibitively expensive to compute. Alternatively, we can estimate PSFs from the data-misfit Hessian operator (Fichtner and van Leeuwen, 2015). From the definition of R, we observe that

$$
\delta \mathbf{m}_{\text {est }}=\mathbf{R} \delta \mathbf{m}_{\text {true }}=\left(\mathbf{H}_{\text {misfit }}+\boldsymbol{\Gamma}_{\text {prior }}^{-1}\right)^{-1} \mathbf{H}_{\text {misfit }} \delta \mathbf{m}_{\text {true }} .
$$

Here, the term $\mathbf{H}_{\text {misfit }} \delta \mathbf{m}_{\text {true }}$ indicates the direction of the model updates. When this is multiplied by $\left(\mathbf{H}_{\text {misfit }}+\boldsymbol{\Gamma}_{\text {prior }}^{-1}\right)^{-1}$, we converge to the solution in a single iteration. If we apply $\mathbf{H}_{\text {misfit }}$ to a point-localized model perturbation $\delta \mathbf{m}$, it will therefore provide a conservative estimation of the PSFs. The eigenvectors corresponding to the largest eigenvalues will obscure the contribution of other eigenvectors that the inverse operator can resolve. In our study, we use this approach for the resolution analysis of the finitefrequency traveltime tomography introduced in Chapter 5. One could attempt to quantify the spatial resolution by considering Gaussian approximations of PSFs and taking their full width half maximum values (e.g., Fichtner and van Leeuwen, 2015). In this thesis, however, due to the simplifications introduced in the forward modelling and the lack of modelling error estimations, we interpret our results in a qualitative manner.

\subsection{Comments on waveform tomography}

The meaning of waveform tomography appears to differ depending on the discipline in which is applied. In seismology, (full) waveform inversion (FWI) refers to the class of techniques that minimize discrepancies between observed and predicted signals (quantified through an objective functional) by numerically solving the wave equation. The method seeks to use the full information content in the observed signals, which are non-linearly related to the physical properties of the media. In general, the solution is estimated using deterministic approaches (e.g., Virieux and Operto, 2009), involving iterative optimization procedures that require numerical wave propagation simulations at each iteration. Here, adjoint techniques provide an elegant and efficient solution to compute Fréchet derivatives of the objective functional with respect to model parameters (Tarantola, 1988b; Tromp et al., 2005; Fichtner et al., 2006; Plessix, 2006). These can be computed by combining two wavefields: a regular wavefield that propagates forward in time from the emitter to the receivers, and an adjoint wavefield that propagates backward in time from the receivers to the emitter. The latter is generated from a source term that depends only on the objective functional.

FWI is inherently a non-linear inverse problem, but there are few instances in which linearized approaches may be justified and applied, similar to Equation (4.5). This generally depends on (1) the convexity of the objective functional that is considered, and/or (2) the relative position of its global minimum with respect to the current model, that is, the model that is being evaluated. Objective functionals that improve the convexity of FWI include, 
for instance, the cross-correlation traveltime shift (Luo and Schuster, 1991; Marquering et al., 1999). This has been already introduced in Section 4.1 .2 and will be described in more detail in the next Chapter 5. In this case, once the method is linearized, it becomes what is commonly referred as finite-frequency tomography. Another field of interest that can benefit from linearized assumptions is the analysis of resolution and uncertainties in FWI. This is useful for optimal experimental design studies, and we discuss it in the following.

When the parameter-to-observable map $\mathbf{F}(\mathbf{m} ; \mathbf{s})$ is non-linear, there are no closed-form expressions for the posterior probability distribution $\pi(\mathbf{m} \mid \mathbf{d} ; \mathbf{s})$. Typically, we rely on Gaussian assumptions to construct local approximations of the posterior in the vicinity of the global minimum, which can be estimated as

$$
\mathbf{m}_{\mathrm{est}}=\underset{\mathbf{m}}{\arg \min }\left\{\chi(\mathbf{m}):=\frac{1}{2}\left\|\mathbf{d}_{\mathrm{obs}}-\mathbf{F}(\mathbf{m} ; \mathbf{s})\right\|_{\boldsymbol{\Gamma}_{\text {noise }}^{-1}}^{2}+\frac{1}{2}\left\|\mathbf{m}-\mathbf{m}_{\text {prior }}\right\|_{\boldsymbol{\Gamma}_{\text {prior }}^{-1}}^{2}\right\} .
$$

Here, we consider the specific case of least-squares minimization and assume Gaussian noise and prior. In the vicinity of $\mathbf{m}_{\text {est }}$, we approximate the objective functional $\chi(\mathbf{m})$ quadratically

$$
\chi(\mathbf{m}) \approx \chi\left(\mathbf{m}_{\mathrm{est}}\right)+\frac{1}{2}\left(\mathbf{m}-\mathbf{m}_{\mathrm{est}}\right)^{T} \mathbf{H}\left(\mathbf{m}-\mathbf{m}_{\mathrm{est}}\right),
$$

where $\mathbf{H}$ is the Hessian of $\chi(\mathbf{m})$ evaluated at $\mathbf{m}_{\text {est }}$, i.e., $\mathbf{H}=\left.\chi^{\prime \prime}(\mathbf{m})\right|_{\mathbf{m}=\mathbf{m}_{\text {est }}}$, and the gradient satisfies $\left.\chi^{\prime}(\mathbf{m})\right|_{\mathbf{m}=\mathbf{m}_{\text {est }}}=0$. We substitute this approximation into the expression of the posterior given in Equation (4.31)

$$
\pi(\mathbf{m} \mid \mathbf{d} ; \mathbf{s}) \propto \exp [-\chi(\mathbf{m})] \propto \exp \left[-\frac{1}{2}\left(\mathbf{m}-\mathbf{m}_{\mathrm{est}}\right)^{T} \mathbf{H}\left(\mathbf{m}-\mathbf{m}_{\mathrm{est}}\right)\right] .
$$

The comparison of Equations (4.61) and (4.32) suggests that, in the vicinity of $\mathbf{m}_{\text {est }}$, the posterior covariance matrix $\boldsymbol{\Gamma}_{\text {post }}$ can be approximated by the inverse of the Hessian $\mathbf{H}$. Therefore, $\mathbf{H}$ contains crucial information on uncertainties and parameter trade-offs, at least when $\mathbf{m}$ is sufficiently close to the global minimum.

From Equation (4.59), we find the explicit expression of $\mathbf{H}$ that results in

$$
\mathbf{H}=\chi^{\prime \prime}(\mathbf{m})=\mathbf{F}^{\prime}(\mathbf{m} ; \mathbf{s})^{T} \boldsymbol{\Gamma}_{\text {noise }}^{-1} \mathbf{F}^{\prime}(\mathbf{m} ; \mathbf{s})+\left(\mathbf{d}_{\text {obs }}-\mathbf{F}(\mathbf{m} ; \mathbf{s})\right)^{T} \mathbf{F}^{\prime \prime}(\mathbf{m} ; \mathbf{s})+\boldsymbol{\Gamma}_{\text {prior }}^{-1} .
$$

Here, $\mathbf{F}^{\prime}$ is the Jacobian operator and contains sensitivities computed from adjoint techniques. $\mathbf{F}^{\prime \prime}$ encodes derivatives of the sensitivities, which may be prohibitive to compute. To circumvent this, we can use second-order adjoint techniques. They allow us to compute Hessian-vector products $\mathbf{H} \delta \mathbf{m}$ at the cost of two additional wave propagation simulations per each $\delta \mathbf{m}$. We refer the reader to Fichtner and Trampert (2011a) for an exhaustive description of the method. In particular, Hessian-vector products are useful to efficiently approximate properties of the Hessian using stochastic approaches (Crestel et al., 2017; 
Martin et al., 2012; Haber et al., 2008; Alexanderian et al., 2016).

In the context of optimal experimental design, the computation of the full $\mathbf{H}$ may still be too expensive, and various studies have relied on the Gauss-Newton approximation to access specific properties of it (e.g., Maurer et al., 2017). The Hessian is approximated as

$$
\mathbf{H}_{\mathrm{GN}}=\mathbf{F}^{\prime}(\mathbf{m} ; \mathbf{s})^{T} \boldsymbol{\Gamma}_{\text {noise }}^{-1} \mathbf{F}^{\prime}(\mathbf{m} ; \mathbf{s})+\boldsymbol{\Gamma}_{\text {prior }}^{-1}
$$

which provides sufficiently accurate approximations of $\mathbf{H}$ when data-misfit residuals $\mathbf{d}_{\mathrm{obs}}-$ $\mathbf{F}(\mathbf{m} ; \mathbf{s})$ are small (Crestel et al., 2017). In addition to being computationally more tractable, $\mathbf{H}_{\mathrm{GN}}$ is, by construction, positive definite at any evaluation point, whereas, for $\mathbf{H}$, this is only guaranteed in the vicinity of $\mathbf{m}_{\text {est }}$. This may be crucial to relax the evaluation point $\mathbf{m}$, while still preserving relevant information about the objective functional (Crestel et al., 2017). Indeed, approximating the Hessian using the Gauss-Newton formulation is equivalent to linearizing the forward operator in the neighborhood of $\mathbf{m}_{\text {est }}$, i.e.,

$$
\mathbf{F}(\mathbf{m} ; \mathbf{s}) \approx \mathbf{F}\left(\mathbf{m}_{\mathrm{est}} ; \mathbf{s}\right)+\left.\mathbf{F}^{\prime}(\mathbf{m} ; \mathbf{s})\right|_{\mathbf{m}=\mathbf{m}_{\mathrm{est}}}\left(\mathbf{m}-\mathbf{m}_{\mathrm{est}}\right)
$$

which is essentially the same as Equation (4.5). 


\section{Finite-frequency tomography ${ }^{\dagger}$}

As already discussed, USCT holds great potential for 3D quantitative imaging of acoustic breast tissue properties. However, typical devices include high-frequency transducers, which makes tomography techniques based on numerical wave propagation simulations computationally challenging, especially in 3D. Therefore, despite the finite-frequency nature of ultrasonic waves, ray-theoretical approaches to transmission tomography are still widely used.

This chapter introduces finite-frequency traveltime tomography to medical ultrasound. In addition to being computationally tractable for 3D imaging at high frequencies, the method has two main advantages: (1) It correctly accounts for the frequency dependence and volumetric sensitivity of traveltime measurements, which are related to off-ray-path scattering and diffraction. (2) It naturally enables out-of-plane imaging and the construction of 3D images from 2D slice-by-slice acquisition systems.

Our approach rests on the availability of calibration data in water, used to linearize the forward problem and to provide analytical expressions of cross-correlation traveltime sensitivity. As a consequence of the finite frequency content, this is distributed in multiple Fresnel volumes, thereby providing out-of-plane sensitivity. To improve computational efficiency, we develop a memory-efficient implementation by encoding the Jacobian operator with a 1D parameterization, which allows us to extend the method to large-scale domains.

In what follows, we first introduce the theoretical aspects of the finite-frequency tomography method, and we suggest efficient implementations in the context of USCT. We then validate our tomographic approach using lab measurements collected with a $2 \mathrm{D}$ setup of transducers and using a cylindrically symmetric phantom. This is followed by a demonstration of its applicability for 3D reconstructions by simulating a slice-by-slice acquisition system using the same dataset. Here we analyze the resolution limits of our approach and we relate this to the design of the acquisition systems. Finally, we conclude the chapter with a discussion about possible extensions of the method to increase the amount of useful information extracted from the signals, and therefore, improve tomographic resolution.

\footnotetext{
${ }^{\dagger}$ The content of this chapter has recently been published as: N. Korta Martiartu, C. Boehm and A. Fichtner (2020). 3D Wave-Equation-Based Finite-Frequency Tomography for Ultrasound Computed Tomography. IEEE Transactions on Ultrasonics, Ferroelectrics, and Frequency Control.
} 


\subsection{Finite-frequency traveltime tomography}

\subsubsection{Traveltime Fréchet derivatives}

Let us start this section reminding the acoustic wave equation formulated in the time domain, for loss-less media and homogeneous density:

$$
\frac{1}{c^{2}(\mathbf{x})} \partial_{t}^{2} p(\mathbf{x}, t)-\nabla^{2} p(\mathbf{x}, t)=f(\mathbf{x}, t)
$$

This equation defines the relationship between our observables and the unknown model parameters $\mathbf{m}=c(\mathbf{x})$, for any arbitrary source $f$, and it will be useful for the subsequent derivations.

Typically, velocity information is retrieved from the first-arrival traveltimes of the recorded signals $p\left(\mathbf{x}_{r}, t ; \mathbf{x}_{s}\right)$ at receiver locations $\mathbf{x}_{r}$. This can be formulated as a leastsquares problem, minimizing the sum of the squared residuals

$$
\Delta T\left(\mathbf{x}_{r}, \mathbf{x}_{s} ; \mathbf{m}\right)=T_{\mathrm{obs}}\left(\mathbf{x}_{r}, \mathbf{x}_{s}\right)-T\left(\mathbf{x}_{r}, \mathbf{x}_{s} ; \mathbf{m}\right)
$$

between the observed and predicted traveltimes for each emitter-receiver combination, respectively. The dependency of $T$ on $\mathbf{m}$ allows us to understand that these traveltimes are estimated through a given physical model. In practice, first-arrival times are measured by the cross-correlation of the observed signals and calibration data. An internally consistent way of formulating the optimization problem is therefore by defining $\Delta T\left(\mathbf{x}_{r}, \mathbf{x}_{s} ; \mathbf{m}\right)$ as the time shift $\tau$ where the cross-correlation

$$
C(\tau)=\int_{t_{0}}^{t_{1}} p_{\text {obs }}\left(\mathbf{x}_{r}, t+\tau ; \mathbf{x}_{s}\right) p\left(\mathbf{x}_{r}, t ; \mathbf{x}_{s}, \mathbf{m}\right) d t
$$

attains its maximum. In our derivations, we closely follow Luo and Schuster (1991). Here, $\left[t_{0}, t_{1}\right]$ is the time interval in which the first arrivals occur, and $p_{\text {obs }}$ and $p$ are the observed and modelled ultrasonic signals, respectively. If we drop the dependencies in $\mathbf{x}_{r}$ and $\mathbf{x}_{s}$ for clarity, $\Delta T$ satisfies

$$
\left.\frac{d C(\tau)}{d \tau}\right|_{\tau=\Delta T}=\int_{t_{0}}^{t_{1}} \partial_{t} p_{\mathrm{obs}}(t+\Delta T) p(t ; \mathbf{m}) d t=0
$$

This equation establishes the implicit relation between $\Delta T$ and $p(t ; \mathbf{m})$. For simplicity, we denote $\left.\partial_{t} p_{\text {obs }}(t+\tau)\right|_{\tau=\Delta T}=\partial_{t} p_{\text {obs }}(t+\Delta T)$. In particular, for the velocity estimations, we are interested in the relationship between the traveltime perturbations $\delta T$ and velocity perturbations $\delta c$. From Equation (5.3), by applying the chain rule for implicit differentiation 
and integration by parts, we obtain

$$
\delta T=\frac{\delta(\Delta T)}{\delta c} \delta c=\frac{1}{N} \int_{t_{0}}^{t_{1}} \partial_{t} p_{\mathrm{obs}}(t+\Delta T) \frac{\partial p(t ; \mathbf{m})}{\partial c} \delta c d t
$$

where the normalization factor is

$$
N=\int_{t_{0}}^{t_{1}} \partial_{t} p_{\text {obs }}(t+\Delta T) \partial_{t} p(t ; \mathbf{m}) d t
$$

The derivatives of the wavefield with respect to the model parameters that appear in Equation (5.4) can be computed from the Born approximation of Equation (5.1) [see Appendix A]. Assume we apply a perturbation in velocity $c \rightarrow c+\delta c$ that causes a firstorder perturbation in the wavefield $p \rightarrow p+\delta p$. Then, the perturbed wavefield $\delta p=\frac{\partial p}{\partial c} \delta c$ is expressed, in terms of Green's function $G$, as

$$
\delta p(\mathbf{x}, t)=\int_{V} \int_{t_{0}}^{t_{1}} \frac{2 \delta c}{c^{3}\left(\mathbf{x}^{\prime}\right)} \partial_{t}^{2} p\left(\mathbf{x}^{\prime}, t^{\prime}\right) G\left(\mathbf{x}, t ; \mathbf{x}^{\prime}, t^{\prime}\right) d t d \mathbf{x}^{\prime}
$$

where $V$ is the volume of our ROI (e.g., Tarantola, 1984). By replacing Equation (5.6) in Equation (5.4), using the reciprocity of Green's function

$$
G\left(\mathbf{x}, t ; \mathbf{x}^{\prime}, t^{\prime}\right)=G\left(\mathbf{x}^{\prime},-t^{\prime} ; \mathbf{x},-t\right),
$$

and expressing the wavefield at $\mathbf{x}_{r}$ as

$$
p\left(\mathbf{x}_{r}, t\right)=\int_{V} p(\mathbf{x}, t) \delta\left(\mathbf{x}-\mathbf{x}_{r}\right) d^{3} \mathbf{x}
$$

we obtain

$$
\delta T\left(\mathbf{x}_{r}, \mathbf{x}_{s}\right)=\int_{V} K\left(\mathbf{x} ; \mathbf{x}_{r}, \mathbf{x}_{s}\right) \delta c(\mathbf{x}) d \mathbf{x} .
$$

Here, $K(\mathbf{x})=K\left(\mathbf{x} ; \mathbf{x}_{r}, \mathbf{x}_{s}\right)$ is the cross-correlation traveltime misfit sensitivity kernel defined as

$$
K(\mathbf{x})=\frac{-2}{c^{3}(\mathbf{x})} \int_{t_{0}}^{t_{1}} \partial_{t} p\left(\mathbf{x}, t ; \mathbf{x}_{s}\right) \partial_{t} p^{\dagger}\left(\mathbf{x}, t_{1}-t ; \mathbf{x}_{r}\right) d t .
$$

This is produced by the interaction between two wavefields: the wavefield $p$ propagating forward from the emitters $\mathbf{x}_{s}$, and the adjoint wavefield

$$
p^{\dagger}(\mathbf{x}, t)=\frac{1}{N} \int_{t_{0}}^{t_{1}} \partial_{t} p_{\mathrm{obs}}\left(\mathbf{x}_{r}, t^{\prime}+\Delta T\right) G\left(\mathbf{x}, t ; \mathbf{x}_{r}, t^{\prime}\right) d t^{\prime}
$$

propagating backward in time from the receivers $\mathbf{x}_{r}$.

Equation (5.9) establishes the relationship between cross-correlation traveltime shifts and velocity perturbations in terms of the finite-frequency sensitivity kernels $K(\mathbf{x})$ (Luo 


\section{Finite-frequency tomography}

and Schuster, 1991; Marquering et al., 1999; Dahlen et al., 2000). That is, it is the explicit expression of the first-order Fréchet derivative of finite-frequency traveltimes with respect to velocity. In principle, the sensitivity kernel in Equation (5.10) depends on both the observables and model parameters, and it is therefore a non-linear relationship. However, linearized approaches can be justified for velocity contrasts up to $10 \%$ with respect to the background model (Mercerat and Nolet, 2012).

\subsubsection{Linearization}

Assume that we represent the velocity distribution of the breast tissue as $c(\mathbf{x})=c_{0}+\delta c(\mathbf{x})$, where $c_{0}$ refers to a homogeneous background model (water). If the differences are small such that $|\delta c(\mathbf{x})| \ll c_{0}$, the observed wavefield $p_{\text {obs }}=p_{0}+\delta p$ will be a time shifted version of the background wavefield $p_{0}$,

$$
p_{\text {obs }}\left(\mathbf{x}_{r}, t+\Delta T\right) \approx p_{0}\left(\mathbf{x}_{r}, t\right) .
$$

For a homogeneous unbounded medium, 3D Green's function is given by

$$
G_{0}\left(\mathbf{x}_{r}, t ; \mathbf{x}_{s}, t^{\prime}\right)=\frac{1}{4 \pi R_{s r}} \delta\left(t-t^{\prime}-\frac{R_{s r}}{c_{0}}\right),
$$

where $R_{s r}=\left\|\mathbf{x}_{s}-\mathbf{x}_{r}\right\|$, and assuming point sources, $f(\mathbf{x}, t)=f(t) \delta\left(\mathbf{x}-\mathbf{x}_{s}\right)$, it follows that

$$
p_{0}\left(\mathbf{x}_{r}, t ; \mathbf{x}_{s}\right)=\frac{1}{4 \pi R_{s r}} f\left(t-\frac{R_{s r}}{c_{0}}\right) .
$$

Upon inserting Equations (5.12) - (5.14) into Equation (5.10), we obtain the explicit expression of the linearized traveltime sensitivity kernel $K_{0}$,

$$
\begin{aligned}
K_{0}(\mathbf{x}) & =\frac{A(\mathbf{x})}{N_{L}} \int_{t_{0}}^{t_{1}} g_{1}(\mathbf{x}, t) g_{2}(\mathbf{x}, t) d t \\
g_{1}(\mathbf{x}, t) & =\partial_{t} f\left(t-\frac{R_{x s}}{c_{0}}\right) \\
g_{2}(\mathbf{x}, t) & =\partial_{t}^{2} f\left(t_{1}-t-\frac{R_{x r}+R_{s r}}{c_{0}}\right), \\
B(\mathbf{x}) & =\frac{-R_{s r}}{2 \pi c_{0}^{3} R_{x s} R_{x r}}, \\
N_{L} & =\int_{t_{0}}^{t_{1}} \partial_{t} f^{2}\left(t-\frac{R_{s r}}{c_{0}}\right) d t
\end{aligned}
$$

where $R_{x s}=\left\|\mathbf{x}-\mathbf{x}_{s}\right\|$ and $R_{x r}=\left\|\mathbf{x}-\mathbf{x}_{r}\right\|$.

Equations (5.15a) - (5.15e) define the key ingredient of the forward operator of finitefrequency traveltime tomography, which does neither depend on the unknown model parameters nor on the observed data. Instead, it requires the source-time function $f(t)$, and this 
can be known, for instance, from the calibration of the scanning device with measurements in water. An example of the finite-frequency sensitivity kernel for a band-limited pulse with frequencies in the range of $1-3 \mathrm{MHz}$ is shown in Figure 5.1. Here, for comparison, we also illustrate the equivalent sensitivity predicted from ray theory (dashed line). Unlike the ray-theoretical sensitivities, which are confined to infinitesimally narrow paths, finite-frequency sensitivities extend to finite volumes away from the geometrical ray. They have an ellipsoidal shape defined by the Fresnel zones, with the strongest contribution coming from the first Fresnel zone. Here, the negative sign indicates that a positive velocity perturbation will produce earlier first arrivals. For higher-order Fresnel zones, the sensitivities are oscillatory with alternating signs, and their magnitude decrease due to destructive interferences between the contribution of individual frequencies. Contrary to ray theory, the sensitivities along the geometrical ray path are zero, which is an effect of the cross-correlation measurements. Here, the entire waveform of the first arrivals contributes to the measured traveltimes, and it is therefore the results of the interference between direct and scattered waves (Marquering et al., 1999). Higher frequencies, however, will produce narrower sensitivity kernels, being consistent with what ray-theory predicts. We refer the reader to (Nolet , 2008) for a more detailed discussion about the sensitivity kernels.

Our approach uses a homogeneous background model to linearize the parameter-toobservable map. This allows us to obtain analytical expressions for the sensitivity kernels. Finite-frequency tomography, however, can similarly be defined for heterogeneous background media, using (5.10) and (5.11) to numerically compute the sensitivities. This can be useful, for instance, to incorporate refraction effects into the method (e.g., Dahlen et al., 2000; Hung et al., 2000).

\subsubsection{D approximation}

When the emitters and receivers are located in the same plane, 2D approximations are often applied to accelerate the reconstructions ( $\mathrm{Li}$ et al., 2014). Assume we discretize the breast tissue using the Cartesian coordinates $\mathbf{x}=[x ; y ; z]$ with the z-direction orthogonal to the acquisition plane. If the breast tissue shows smooth variations of the velocity in z-direction, at least in the volumes defined by the sensitivity kernels, then $\delta c(\mathbf{x}) \approx \delta c(x, y)$, and the forward problem can be reduced to

$$
\delta T\left(\mathbf{x}_{r}, \mathbf{x}_{s}\right) \approx \iint_{S}\left(\int_{z_{0}}^{z_{1}} K_{0}\left(\mathbf{x} ; \mathbf{x}_{r}, \mathbf{x}_{s}\right) d z\right) \delta c(x, y) d x d y
$$

where $S$ is the ROI located at the acquisition plane, and $\left[z_{0}, z_{1}\right]$ is the interval in which the main contribution of $K_{0}$ occurs. The accuracy of this approximation will depend on both the structure of the breast tissue and the frequencies used. At high frequencies, the sensitivity kernels extend to narrower volumes, and the assumption of smooth variations 


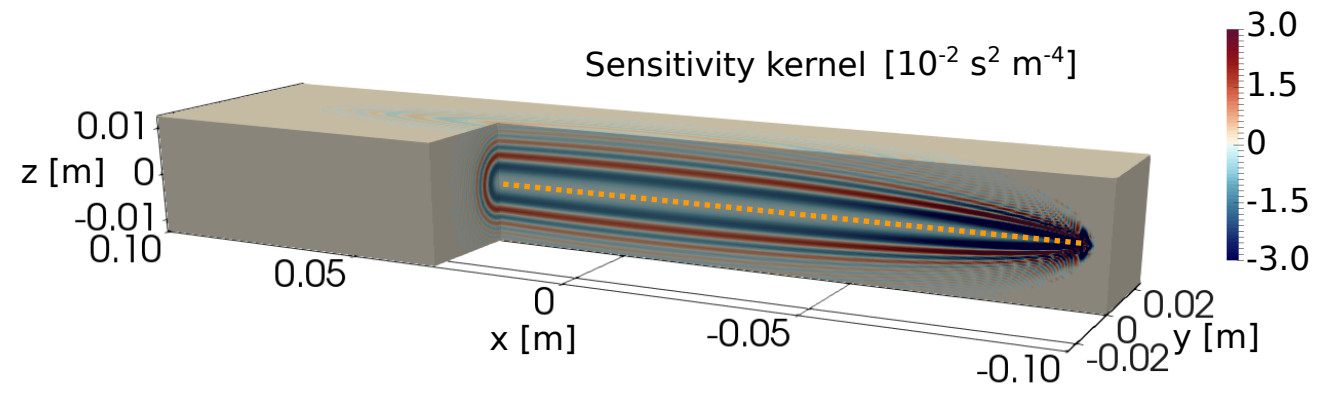

Figure 5.1.: Sensitivity kernel for a band-limited signal with frequencies in the range of 1-3 MHz. The emitter and the receiver are located at positions $(-95,0,0) \mathrm{mm}$ and $(95,0,0) \mathrm{mm}$, respectively. The dashed line indicates the corresponding sensitivity predicted from ray theory.

can be better justified.

\subsubsection{New parameterization of the forward operator}

The combination of Equations (5.9) and (5.15), or (5.16) for 2D approximations, describes the tomographic method presented in this study. Using a compact notation, the linearized forward problem is written as

$$
\delta \mathbf{T}=\mathbf{F}^{\prime} \delta \mathbf{c}
$$

where $\mathbf{F}^{\prime} \in \mathbb{R}^{M \times N}$ is the forward matrix, $\delta \mathbf{T}=\left[\delta T_{1} ; \ldots ; \delta T_{M}\right]$, and $\delta \mathbf{c}=\left[\delta c_{1} ; \ldots ; \delta c_{N}\right]$. Here, $M$ and $N$ indicate the number of measurements and model parameters, respectively. Each row in $\mathbf{F}^{\prime}$ corresponds to a sensitivity kernel for an emitter-receiver pair. As Figure 5.1 suggests, this forward matrix is denser than its equivalent in ray theory. For large-scale problems, the explicit computation of $\mathbf{F}^{\prime}$ may even become prohibitive due to large memory requirements. We circumvent this by encoding the information contained in the sensitivity kernels using a new parameterization. This allows us to solve the inverse problem related to Equation (5.17) using iterative solvers as, for instance, the LSQR algorithm Paige and Saunders (1982b), in which the matrix $\mathbf{F}^{\prime}$ is implicitly given through matrix-vector products.

The pattern shared by the sensitivity kernels can easily be observed in the temporal Fourier domain. We first convert Equations (5.4) and (5.5) with the approximation made in Equation (5.12) (Tong et al., 1998b; Nolet, 2008):

$$
\delta T=-\frac{\operatorname{Re} \int_{0}^{\infty} i \omega p(\omega)^{*} \delta p(\omega) d \omega}{\int_{0}^{\infty} \omega^{2} p(\omega)^{*} p(\omega) d \omega}
$$

Here, $p(\omega)$ denotes the Fourier-transformed pressure field, with angular frequency $\omega$, and we omitted the spatial dependency for clarity. The superscript $*$ and Re denote the complex conjugate and the real part of the complex number, respectively. 
For a point source $f(\mathbf{x}, \omega)=f(\omega) \delta\left(\mathbf{x}-\mathbf{x}_{s}\right)$, the pressure fields at receiver locations $\mathbf{x}_{r}$ can be expressed in terms of Green's functions as

$$
\begin{gathered}
p(\omega)=f(\omega) G_{0}\left(\mathbf{x}_{r}, \omega ; \mathbf{x}_{s}\right), \\
\delta p(\omega)=-\int_{V} \frac{2 \omega^{2} \delta c\left(\mathbf{x}^{\prime}\right)}{c_{0}^{3}} p\left(\mathbf{x}^{\prime}, \omega ; \mathbf{x}_{s}\right) G_{0}\left(\mathbf{x}_{r}, \omega ; \mathbf{x}^{\prime}\right) d \mathbf{x}^{\prime},
\end{gathered}
$$

where frequency-domain Green's function is

$$
G_{0}\left(\mathbf{x}_{r}, \omega ; \mathbf{x}_{s}\right)=\frac{1}{4 \pi R_{s r}} \exp \left(-i \frac{\omega}{c_{0}} R_{s r}\right) .
$$

Upon inserting Equation (5.19) - (5.21) into Equation (5.18), the sensitivity kernel $K_{0}=$ $K_{0}(\mathbf{x})$ takes the form

$$
K_{0}=B(\mathbf{x}) \frac{\int_{0}^{\omega} \omega^{3}|f(\omega)|^{2} \sin \left(\frac{\omega}{c_{0}}\left(R_{x s}+R_{x r}-R_{s r}\right)\right) d \omega}{\int_{0}^{\omega} \omega^{2}|f(\omega)|^{2} d \omega} .
$$

The term $B(\mathbf{x})$ is the same as in Equation $(5.15 \mathrm{~d})$, and it mostly accounts for geometrical spreading. Because it does not depend on $\omega$, it can be included in the sensitivity kernels at a later stage. We therefore do not consider it for the new parameterization of the forward operator.

From Equation (5.22), we see that by defining $K_{0}$ in terms of $R=R_{x s}+R_{x r}-R_{s r}$, the sensitivities for any emitter-receiver combination are represented by the same analytical function. We show an example of this in Figure 5.2. The function describes the diffraction pattern observed in the sensitivity kernels, that is, the values and locations of the Fresnel zones. Being independent of the emitter-receiver geometry, it essentially encodes the complete forward operator $\mathbf{F}^{\prime}$, without the need of computing it explicitly. Usually, we need very few terms to represent accurately the function in Equation (5.22), and the sparsity of the forward operator can be controlled by truncating the maximum value of the parameter $R$. We therefore store the coefficients and arguments of the involved trigonometric functions, and we compute the elements of $\mathbf{F}^{\prime}$ on the fly through the matrix-vector products required during the iterative linear inversion. For each matrix-vector operation, the actual values of the sensitivities are computed by evaluating the analytical function in the discretization grid, and multiplying them by the corresponding geometrical spreading term $B(\mathbf{x})$. These computations can be done very efficiently in GPU architectures, for which trigonometric functions are optimized operations. 

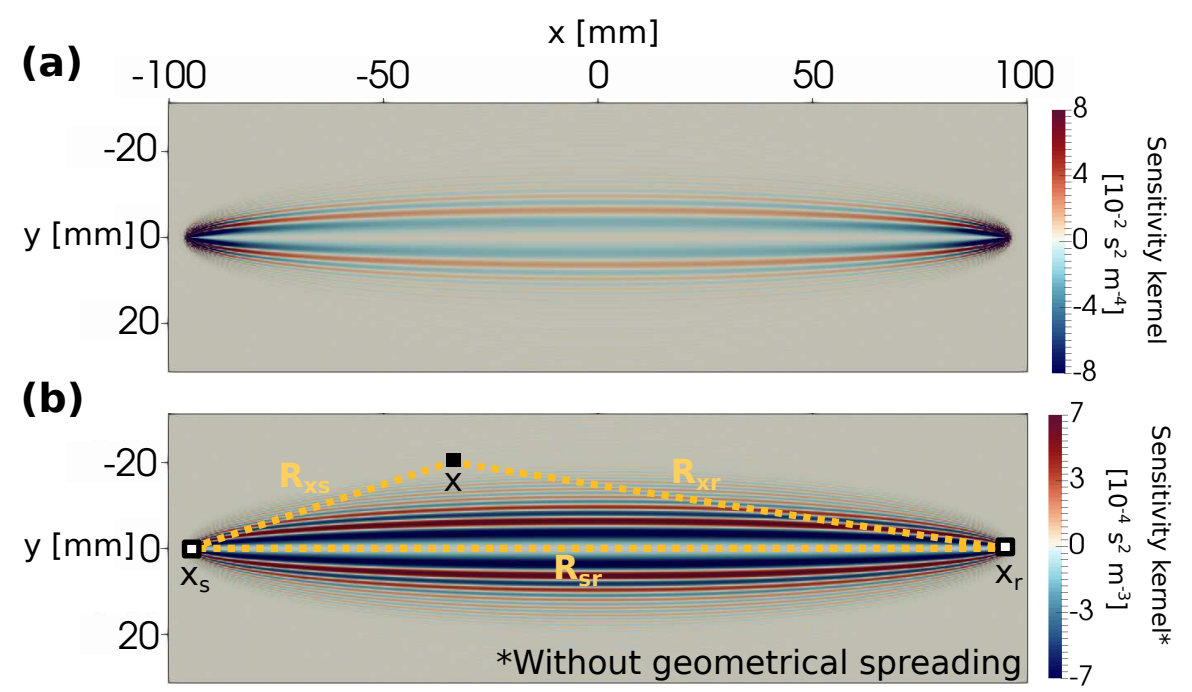

(c)

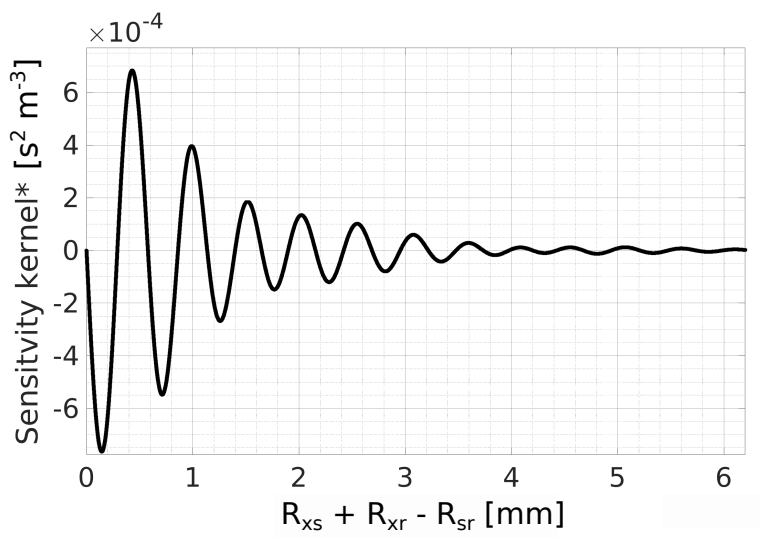

Figure 5.2.: Cross-section in xy-plane of the sensitivity kernel in Figure 5.1 (a) with and (b) without the geometrical spreading term $B(\mathrm{x})$. (c) Sensitivity kernel in (b) represented as a function of $R=R_{x s}+R_{x r}-R_{s r}$. These distances are indicated in (b) with dashed lines, together with the positions of the emitter $\mathbf{x}_{s}$, receiver $\mathbf{x}_{r}$ and an arbitrary spatial location $\mathbf{x}$. 


\subsection{D lab data application}

We consider the dataset provided by the Spanish National Research Council (CSIC) and the Complutense University of Madrid (UCM) as part of the SPIE USCT Data Challenge 2017 (Ruiter et al., 2018; Camacho et al., 2012). The experimental setup used for the measurements is shown in Figure 5.3. The acquisition system consists of two 16-element linear transducer arrays. The elements in one array act as emitters with a central frequency of $3.2 \mathrm{MHz}$ and bandwidth of $50 \%$, while the others are receiving. To acquire transmission data, the receiving array is placed in 11 different positions per position of the emitting array. The whole system is rotated 23 times with respect to the vertical axis describing a circle of $95 \mathrm{~mm}$ radius and providing a total of 64768 A-scans. In this way, the emitting array covers $360^{\circ}$ without any gap. The last rotation, however, produces a partial overlap between the first and last positions of the emitting array. The maximum data coverage is obtained in a circular ROI of $70 \mathrm{~mm}$ radius, indicated in gray in Figure 5.3. The same figure also illustrates the cylindrical phantom used for the measurements. It is based on water, gelatine, alcohol and graphite powder, and it includes an homogeneous background of $94 \mathrm{~mm}$ diameter, two inclusions of $20 \mathrm{~mm}$ diameter, and two steel needles of $0.25 \mathrm{~mm}$ diameter. The phantom is submerged in water with a calibrated velocity of $1479.7 \mathrm{~m} / \mathrm{s}$.

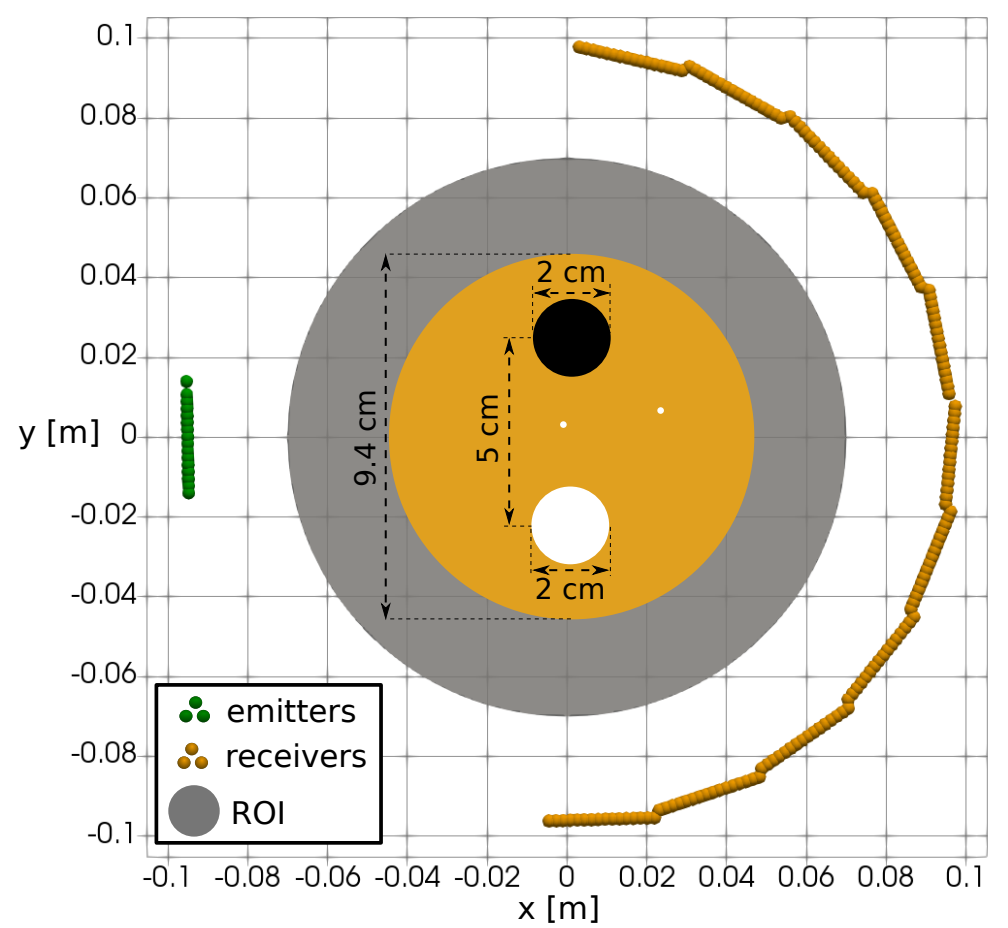

Figure 5.3.: Acquisition system and an illustration of the tissue mimicking phantom used for the lab measurements. The phantom contains two inclusions of low (black circle) and high (white circle) velocity, and two steel needles (white dots). Here the colors do not have a quantitative meaning. 


\section{Finite-frequency tomography}

Although the experiment is inherently $3 \mathrm{D}$, the lack of vertical variation in acoustic properties of the phantom makes 2D approximations reasonable. Figure 5.4(a) shows the velocity reconstruction obtained using the 2D forward problem in Equation (5.16). Here, and hereafter, we apply total variation regularization (Jensen et al., 2012), and we test different values of the regularization parameter to select the most suitable one. This regularization approach is chosen based on the features of the tissue mimicking phantom. We discretize the model parameters using a rectilinear grid with $1 \mathrm{~mm}$ mesh size. This gives a total of 15373 unknowns in the ROI, and thus, a forward operator with dimensions $64768 \times 15373$. Despite the over-determined nature of the problem, redundancies exist due to imperfect data coverage, and the inverse problem is ill-posed [see Figure 5.5(a)]. By summing the absolute values of the rows in the forward operator, we also compute the sensitivity coverage of the experiment, shown in Figure 5.4(b). We observe that the coverage decreases towards the center of the ROI, which is a consequence of the approximately regular distribution of the transducers (Gemmeke et al., 2014; Korta Martiartu et al., 2017). The region with highest coverage in the left side is caused by the overlapped positions of transducers in the last rotation of the acquisition system.

Our reconstruction recovers accurately both velocity heterogeneities and the homogeneous background of the phantom. First-arrival traveltimes do not contain information about the needles, which act as scatterers, and they are therefore invisible for transmission tomography. Empirical velocity measurements of the true phantom are not available, and this excludes a quantitative assessment of our velocity estimations. Yet, our results are in agreement with the reconstructions obtained by other groups (Ruiter et al., 2018), which certainly shows the efficiency of our imaging method. Whereas both inclusions have the same size, a closer look to our reconstruction reveals that the high velocity inclusion is slightly bigger than the low velocity one. This may be an effect of our linearization approximation, in which the sensitivity kernels are computed in an homogeneous model, and therefore, they neglect the bending that occurs in heterogeneous media. Compared to straight-ray tomography, however, the size of the low velocity inclusion is more accurately recovered by our method. A comparison of results obtained by both methods is shown in Chapter 7 [see Figure 7.5].

To assess the quality of our solution, a comparison between the true model and the solution might be insufficient. Although this suggests that the main features of the true phantom are well resolved, it obscures the actual contribution of our prior knowledge introduced by the choice of the regularization. For this example a comprehensive analysis of the resolution is available. We compute the singular value spectrum of the forward operator, shown in Figure 5.5(a). As we observe, the singular values decay rapidly in magnitude, suggesting that the data only contain information about few effective model parameters. We truncate the singular value spectrum after a decrease of three orders of magnitude and consider values below as the effective nullspace. In this way, the truncated 

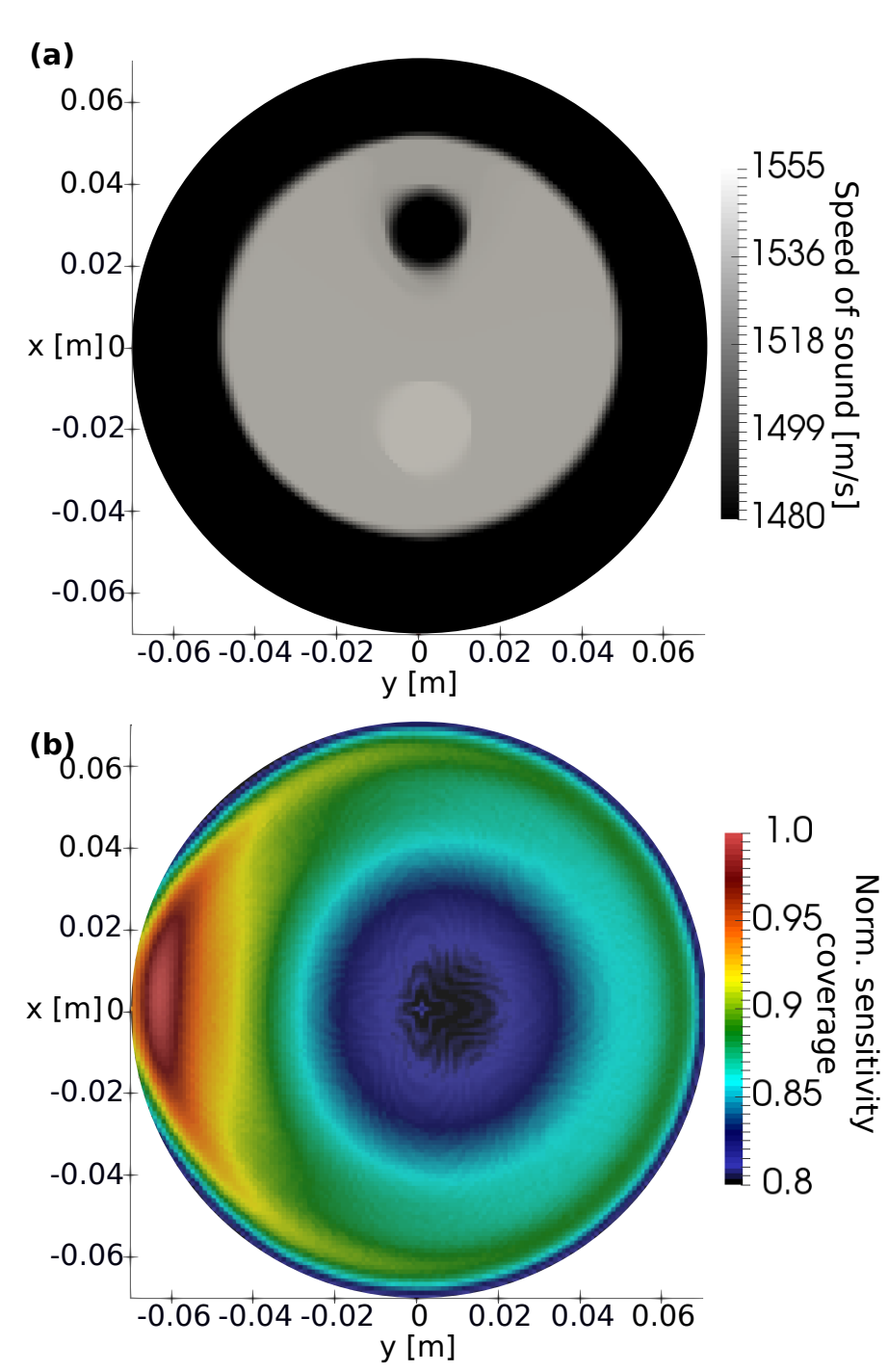

Figure 5.4.: (a) 2D velocity reconstruction and (b) normalized sensitivity coverage. Both images only show the ROI indicated in gray in Figure 5.3.

eigenvalues satisfy the condition for appropiate approximations of posterior covariance and resolution analysis [see Equation B.7 in Appendix B]. We therefore retain 3000 singular values, equivalent to a cutoff of $\lambda>0.05$, and we compute low-rank approximations of the posterior covariance and resolution matrices, see Equations (4.55) and (4.56), respectively. Here, we assume equally reliable observations, i.e., $\boldsymbol{\Gamma}_{\text {noise }}=\sigma_{p}^{-2} \mathbf{I}$ with $\sigma_{p}=2.5 \cdot 10^{-8} \mathrm{~s}$ being the minimum cross-correlation time shift that can be measured. The diagonal elements of these matrices are shown in Figures 5.5(b) and 5.5(d), respectively. To better understand the images, we moreover show their cross sections at $y=0 \mathrm{~mm}$ in Figures 5.5(c) and 5.5(e). For both quantities we observe similar features: the parameter resolution decreases gradually, and the variances increase towards the center of the ROI. This means that we are 


\section{Finite-frequency tomography}

more uncertain about the parameters resolved at the center of the ROI, which corresponds to the observations made in the sensitivity coverage.

The diagonal elements of the resolution matrix are useful to understand where we may expect better resolved parameters. However, it misses useful information encoded in the off-diagonal elements about trade-offs and spatial correlations between parameters that occur due to imperfect data coverage. In Figure 5.6(a), we represent few columns of the resolution matrix, which describe the blurring effect introduced by the inverse operator. These are the PSFs associated to point-localized unit perturbations at positions $(x, y)=(0,0),(0,20),(0,40),(0,60),(-60,0),(-40,0),(-20,0) \mathrm{mm}$. Our resolution analysis reveals that the smearing mainly extends to circular areas of $1 \mathrm{~mm}$ radius. The worst resolution is obtained at the center of ROI, and the trade-offs decrease in spatial extension towards its boundary. Similar behaviour is observed in the amplitude of the PSFs.

The information extracted from the resolution matrix corresponds to an idealized situation, and it may lead us to rather optimistic conclusions. It assumes that (1) the observed data satisfies exactly the forward problem in Equation (5.17), and (2) our model estimation fully converges to the solution. For 3D problems the computation of the resolution matrix becomes prohibitive, and therefore, PSFs can only be estimated through Hessian-vector products [see Equation (4.58)]. These quantities, which indicate the direction of the singleiteration model update, are considered as conservative estimations of PSFs (Fichtner and Trampert, 2011b; Fichtner and van Leeuwen, 2015). Here we compare both results in order to gain deeper understanding for the following sections.

The estimations of the PSFs using Hessian-vector products are shown in Figure 5.6(b) for the same locations as before. As expected, the parameter trade-offs extend to wider areas than the ones estimated using the resolution matrix. This may be a consequence of the projection of the $3 \mathrm{D}$ sensitivities to the $\mathrm{x}-\mathrm{y}$ plane, which is intrinsic to our definition of the 2D forward problem [see (5.16)]. Although the trade-offs are mainly localized in a circle of $5 \mathrm{~mm}$ radius around the positions of the perturbations, the locations of the most significant values agree with our observations in Figure 5.6(a). In fact, the principal difference between Figures 5.6(a) and 5.6(b) is due to the normalization factor in Equation (4.58). PSFs estimated by Hessian-vector products are dominated by smoother eigenvectors associated to largest eigenvalues. Consequently, they obscure the small-scale features provided by other eigenvectors that the inverse operator resolves. We therefore interpret Figures 5.6(a) and 5.6(b) as the lower and upper bounds, respectively, of the PSFs expected for a realistic estimation. For a quantitative analysis, however, we would require the accurate understanding of modelling errors introduced by our forward problem formulation. Because this needs further experimental studies, as we discuss in Section 5.5, here we use estimations of PSFs for comparative analysis of the local resolution.

The computation of the low-rank resolution matrix has an additional advantage. By applying it to the solution shown in Figure 5.4(a), which is obtained considering the full 

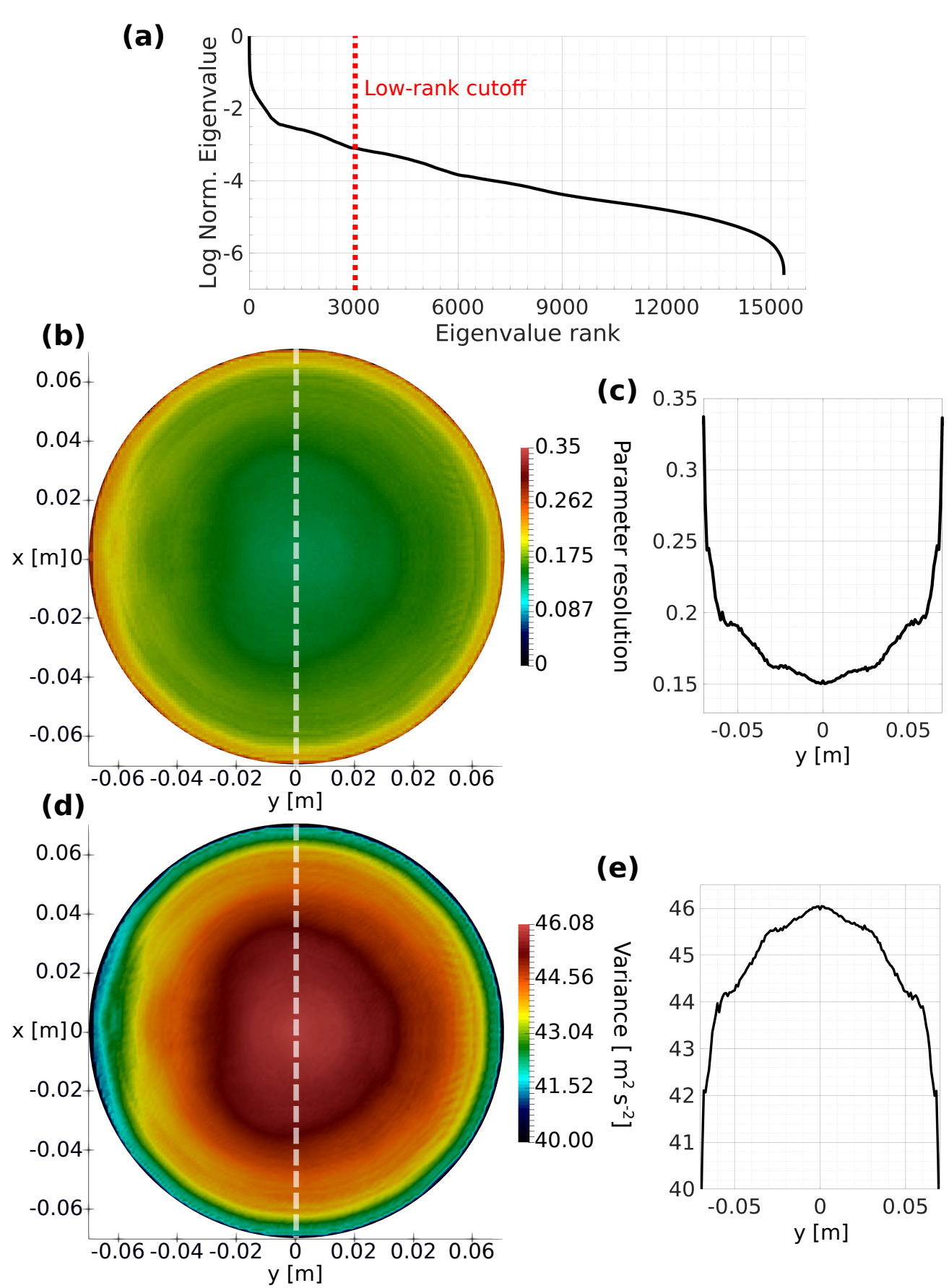

(e)

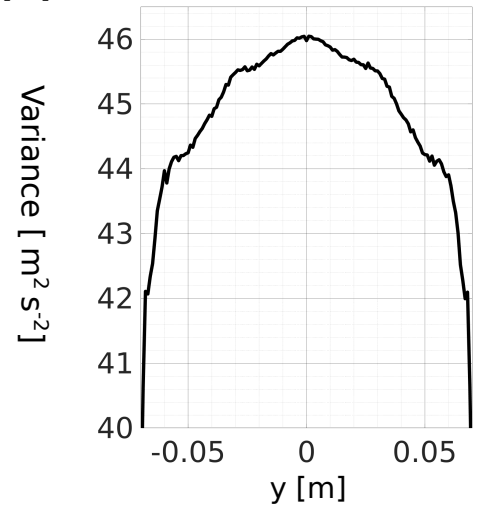

Figure 5.5.: (a) Singular value spectrum of the 2D forward operator. The red line indicates the low-rank cutoff used to approximate the resolution and posterior covariance matrices. (b), (c) Diagonal elements of the resolution matrix and a cross section at the position indicated by the dashed white line, respectively. (d), (e) Diagonal elements of the posterior covariance matrix and a cross section at $y=0 \mathrm{~mm}$, respectively. 


\section{Finite-frequency tomography}

rank, we ideally filter any regularization effect introduced through the effective nullspace of the forward operator. Following Equation (4.57), the solution is projected onto a basis of eigenvectors corresponding to the largest 3000 singular values of $\mathbf{F}^{\prime}$. The resulting model, shown in Figure 5.7, does not include resolved features that are contained in the effective nullspace and therefore illustrates the components of the reconstruction that are mostly explained by the data. As expected, the result shows both oscillatory velocity variations and smoother contrasts. Importantly, it suggests that the recovered size of the phantom and the inclusions are independent of our prior knowledge. 

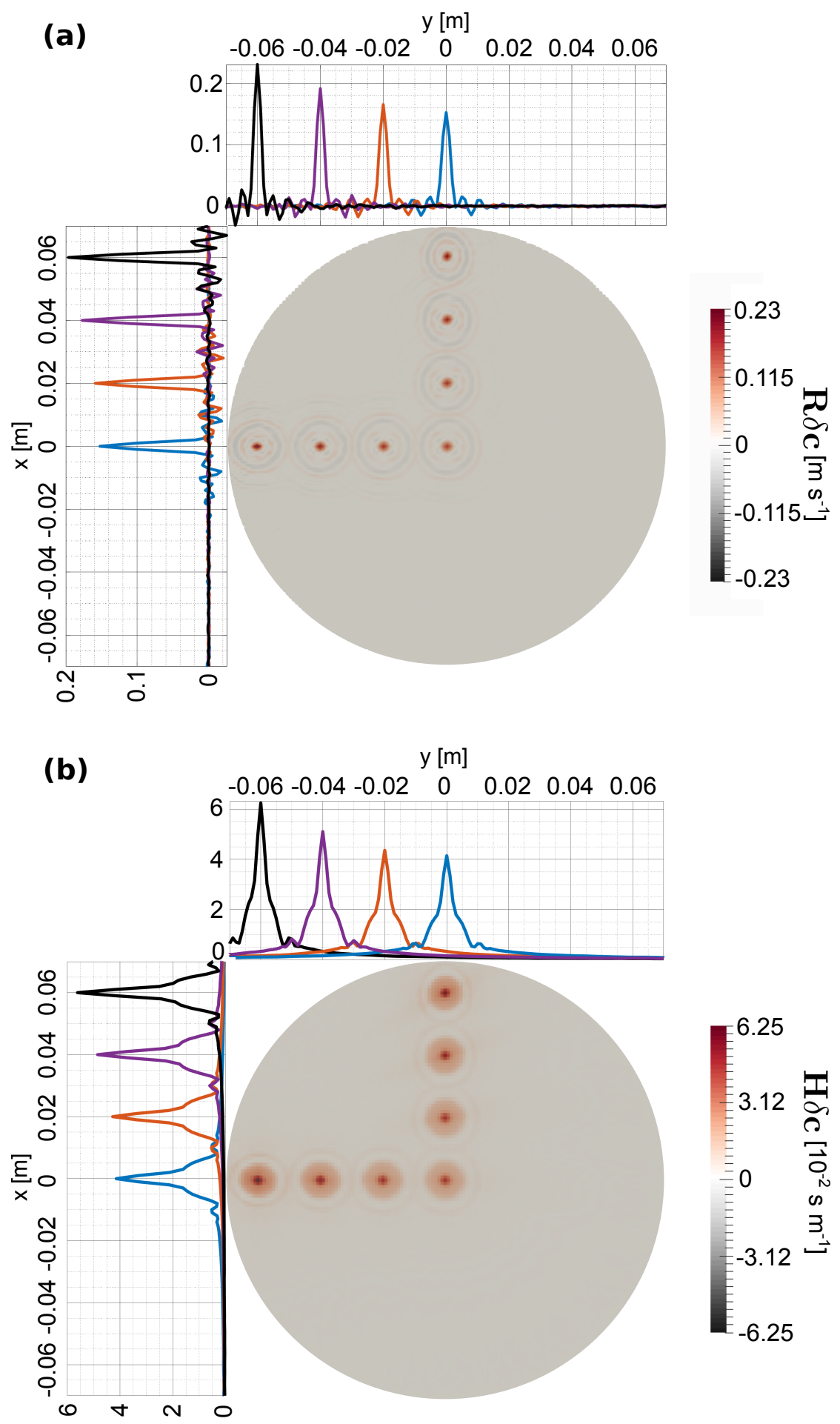

Figure 5.6.: PSFs corresponding to point-localized unit perturbations at positions $(x, y)=$ $(0,0),(0,20),(0,40),(0,60),(-60,0),(-40,0),(-20,0) \mathrm{mm}$. These are estimated using (a) the resolution matrix, and (b) Hessian-vector products. In both figures we show the horizontal and vertical cross sections at $y=0 \mathrm{~mm}$ and $x=0 \mathrm{~mm}$, respectively. 


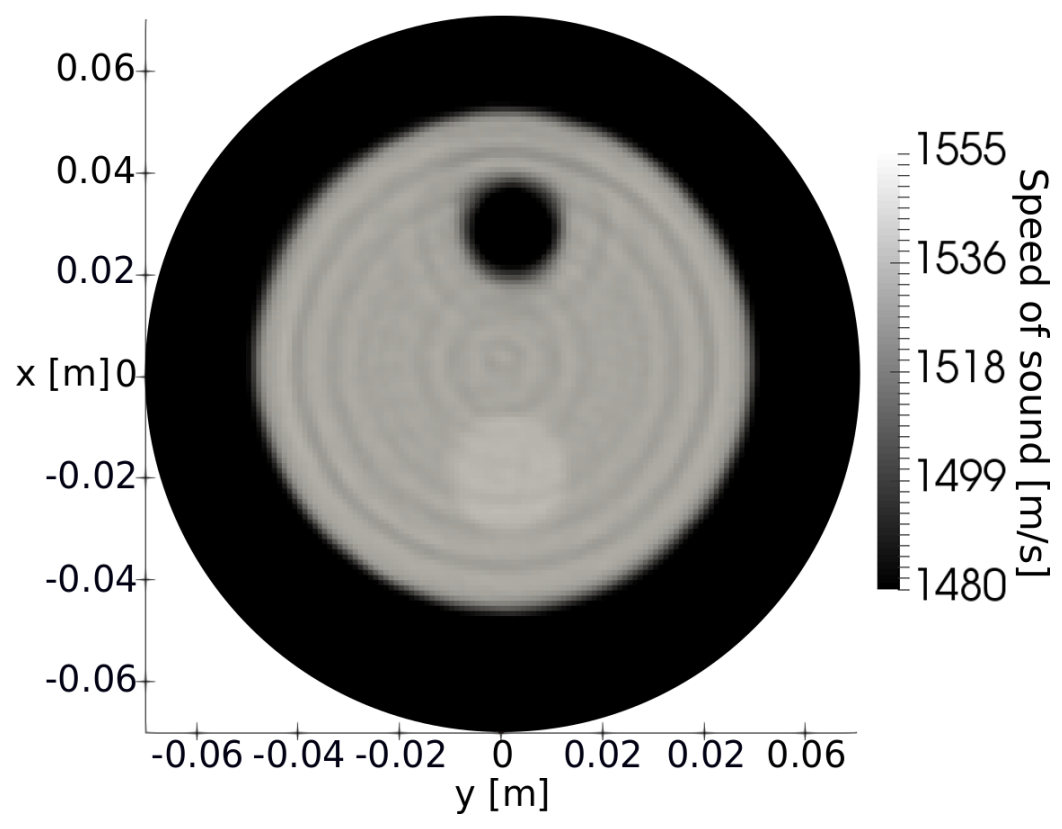

Figure 5.7.: Solution in Figure 5.4(a) projected onto a basis of eigenvectors corresponding to the largest 3000 singular values of $\mathbf{F}^{\prime}$. 


\section{3. $3 \mathrm{D}$ reconstruction with $2 \mathrm{D}$ lab dataset}

Lab experiments are 3D in nature, and volumetric reconstructions may be preferable, especially when the observations are sensitive to regions with vertically varying structure. In this section, we use the same dataset as in the example before to illustrate the potential of our method to image out of plane.

We apply the forward problem in Equation (5.9) to reconstruct the 3D velocity distribution. The result is shown in Figure 5.8(a), and it proves that indeed finite-frequency tomography is capable of providing volumetric images from $2 \mathrm{D}$ acquisition systems. In general, our estimated velocity model recovers the main features of the true phantom, with similar accuracy as in the $2 \mathrm{D}$ example. The vertical width of the reconstruction corresponds to the region with the highest sensitivity coverage. This is controlled by the interaction of the first Fresnel zone of the sensitivities corresponding to each emitter-receiver combination. In this application, the maximum Fresnel width is $\sqrt{\frac{c_{0} L_{\max }}{f_{c}}}=9.4 \mathrm{~mm}$, where $c_{0}=1479.7 \mathrm{~m} / \mathrm{s}$ is the background water velocity, $L_{\max }=190 \mathrm{~mm}$ is the maximum emitter-receiver distance, and $f_{c}=3.2 \mathrm{MHz}$ is the dominant frequency of the emitting signal. The vertical thickness of our reconstruction will therefore be constrained by this value.

To better understand the quality of our reconstruction, we compute a collection of PSFs estimated by Hessian-vector products. These are visualized in Figure 5.8(b) as vertical and horizontal cross sections. The results reveal a similar horizontal resolution as in the 2D example, but a poor vertical resolution that essentially makes the sound-speed model indistinguishable in z-direction. As mentioned before, its extent comprises the most covered region of approximately $8 \mathrm{~mm}$ width, and it remains constant along the horizontal direction. In this example, the lack of crossing sensitivity kernels in z-direction decreases our ability to constrain the parameters independently. 


\section{(a) Reconstruction}

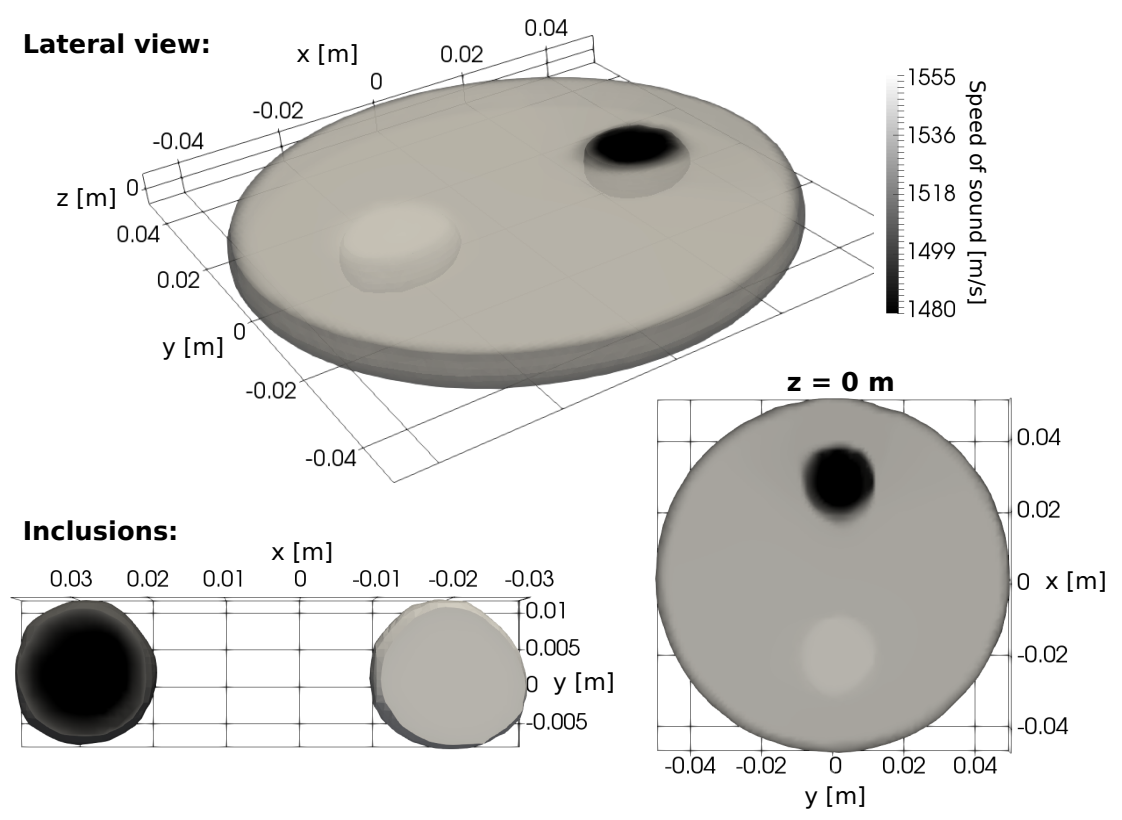

(b) Point-spread functions
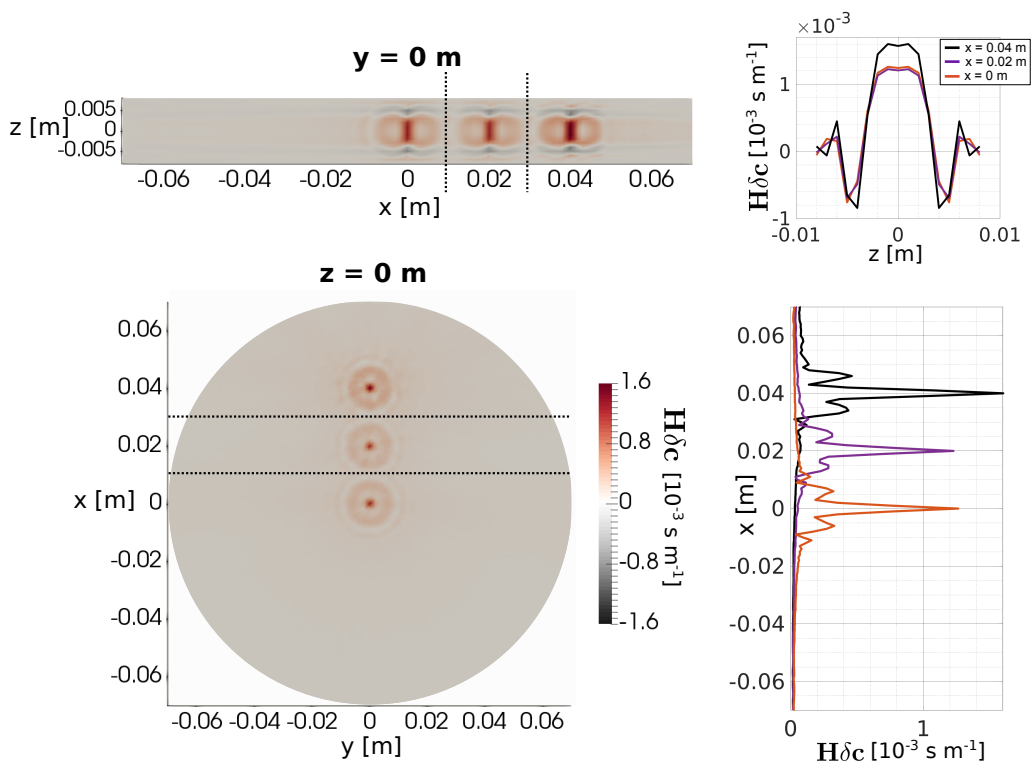

Figure 5.8.: (a) 3D velocity reconstruction using the same dataset as in the $2 \mathrm{D}$ example. We show the lateral view of the volumetric reconstruction, the top view of the isolated inclusions, and a cross section at $z=0 \mathrm{~mm}$. We reduce the opacity of the first image to visualize the inclusions. (b) Estimations of PSFs due to unit perturbations at locations $(x, y, z)=(0,0,0),(20,0,0),(40,0,0) \mathrm{mm}$. We show the 2D and 1D cross sections in vertical and horizontal directions. Dashed lines indicate that PSFs are computed individually, although we visualize all in the same image. 


\subsection{Simulated full 3D experiment: slice-by-slice acquisition}

To improve the vertical resolution of an experiment by constraining better the unknown parameters, it is essential to provide measurements with crossing or overlapping sensitivities. For the previous experiment, this can be done, for instance, by collecting additional measurements at different elevations. Because the phantom has cylindrical symmetry, we simulate a slice-by-slice acquisition, and we assume that same data has been recorded at different elevations. The aim of this example is twofold: on one hand, we want to show that finite-frequency tomography is a powerful tool to provide consistent full 3D images from slice-by-slice acquisition systems; and on the other hand, we want to investigate the conditions for a meaningful vertical resolution. We relate the latter to the vertical spacing between different elevations.

In our previous result, we observed a vertical resolution of approximately $8 \mathrm{~mm}$. Following this, we compare experiments using a vertical spacing of $3 \mathrm{~mm}$ and $8 \mathrm{~mm}$. The first spacing ensures the overlapping of the sensitivities at different elevations, and the second one only avoids gaps between them. Figure 5.9 shows the reconstructions for both cases, in which the ROI is a cylinder with radius $70 \mathrm{~mm}$ and height $32 \mathrm{~mm}$. For Figure 5.9(a) we translate the scanning system to 11 positions in $z \in[-23,23] \mathrm{mm}$, and Figure 5.9 (b) has 7 positions in $z \in[-24,24] \mathrm{mm}$. In both cases, our method successfully recovers the cylindrical 3D phantom, including both heterogeneities. Because current methods using these acquisition systems obtain 3D breast images by stacking 2D reconstructed slices (Duric et al., 2005), our results constitute a fundamental advance in this context.

The experiment with the largest spacing introduces oscillations in the shapes of the recovered heterogeneities. To understand this better, we compute PSFs and analyze differences in local resolution for both experiments, shown in Figure 5.10. As expected, the PSFs computed for $8 \mathrm{~mm}$ spacing are equivalent to those already observed in Figure 5.8. However, the vertical cross sections demonstrate that the resolution length can significantly be reduced when the sensitivities overlap. We illustrate this in Figure 5.10(c), where we plot the $1 \mathrm{D}$ cross sections of the PSFs due to a perturbation at $x=0 \mathrm{~mm}$. The response is remarkably sharper for $3 \mathrm{~mm}$ spacing meaning that the parameters in vertical direction are better constrained. Although we also observe relatively strong side lobes in the responses, these become weaker towards the center of the ROI, where the sensitivities from different elevations overlap most. Horizontal cross-sections also show an interesting effect, see Figure 5.10(d). PSFs for $3 \mathrm{~mm}$ have an increased contrast and trade-offs that decrease in spatial extent. 
(a) Spacing: $3 \mathbf{m m}$
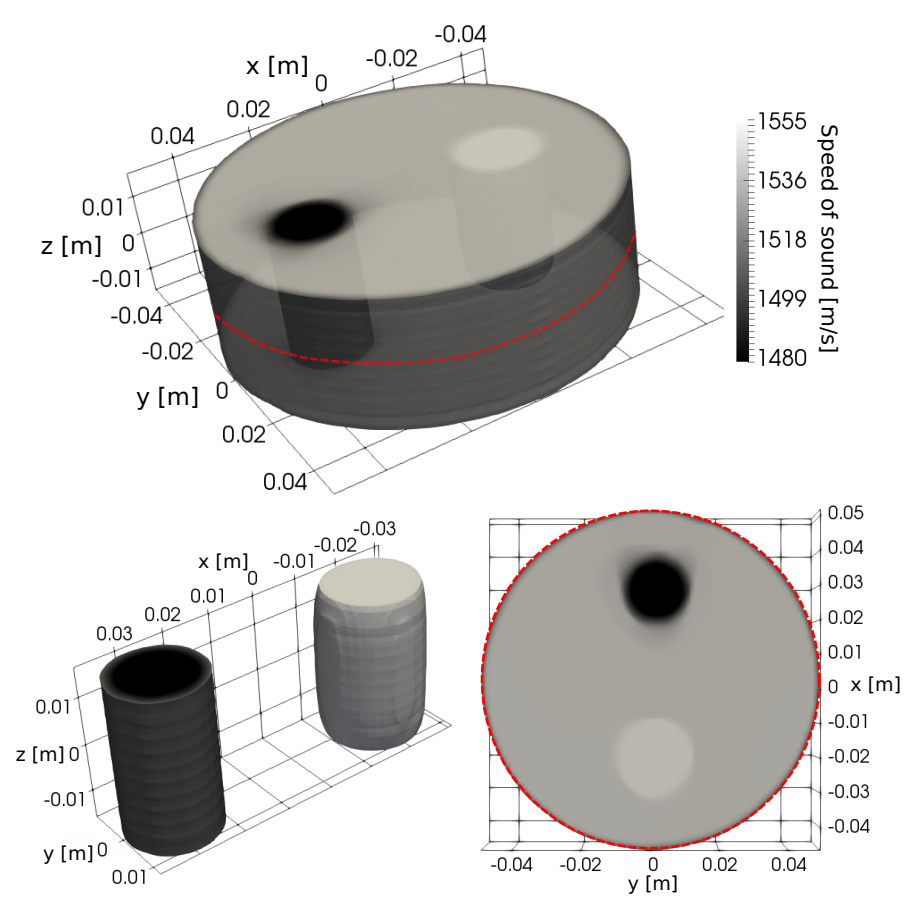

(b) Spacing: $8 \mathrm{~mm}$
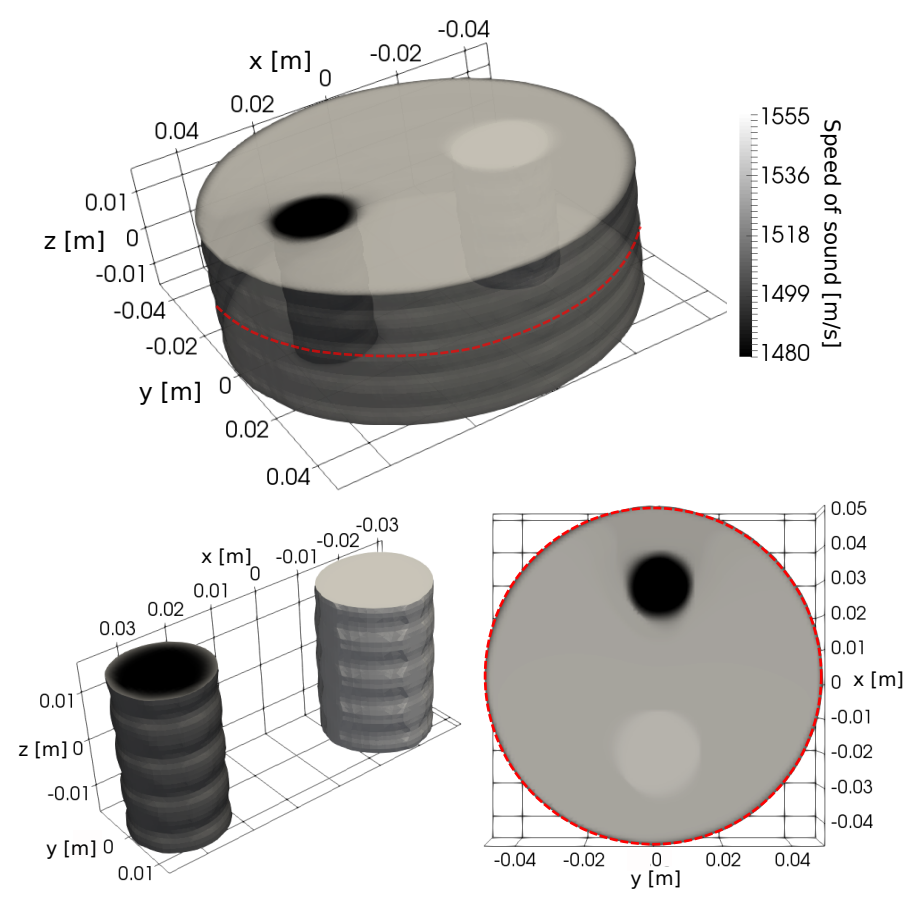

Figure 5.9.: $3 \mathrm{D}$ velocity reconstruction for (a) $3 \mathrm{~mm}$ and (b) $8 \mathrm{~mm}$ spacing between measurements at different elevations. We show in each case the lateral view, the isolated view of the inclusions and the cross section at $z=0 \mathrm{~mm}$. The red lines indicate the positions of the cross sections. 


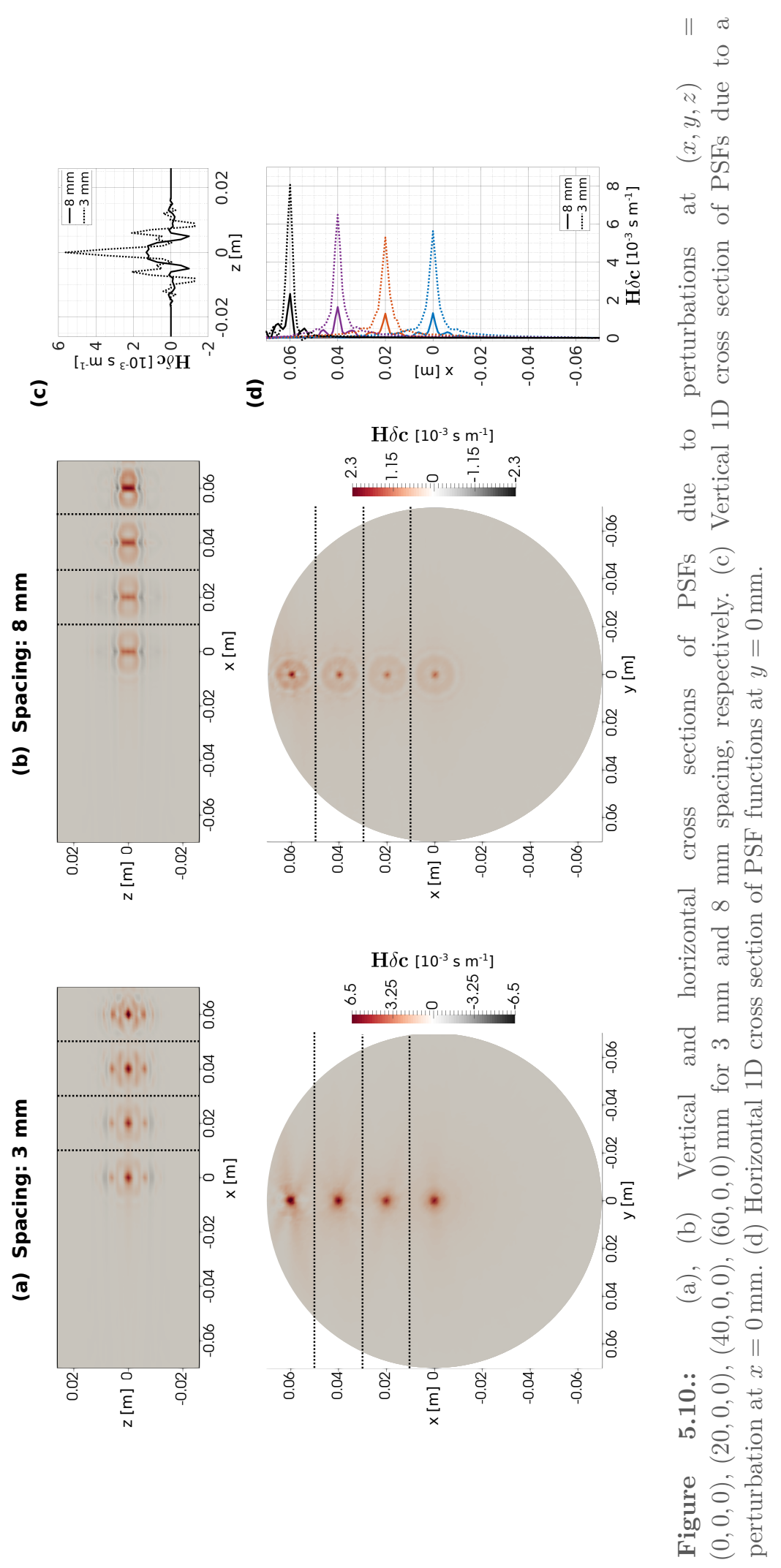




\section{Finite-frequency tomography}

\subsection{Discussion and conclusion}

This work presents a new transmission tomography method to estimate velocity variations in breast tissue using ultrasound data. It minimizes cross-correlation traveltime shifts between the observations and calibration data in water, being internally consistent with the standard procedure of traveltime estimations. The actual measurements are bandlimited and include finite-frequency effects of the cross-correlated pulses. Contrary to what ray theory predicts, finite-frequency traveltimes are affected by scattering and diffraction effects off the ray path, and their sensitivity to velocity variations therefore extends to ellipsoidal volumes. In the context of transmission tomography, this work represents a natural extension of ray theory to finite-frequency waves.

Finite-frequency tomography has been extensively studied in seismology. It is best suited for experiments with (1) velocity variations that are under 10\% (Mercerat and Nolet, 2012) and therefore quasi-linearly related to traveltime differences, (2) sufficient waveform similarity between cross-correlated waves, and (3) dense data coverage. In breast imaging with USCT, all these conditions are satisfied, therefore suggesting an ideal field of application for this method.

Though being methodologically more involved than ray-based tomography, finite-frequency tomography has two major advantages. First and foremost, it correctly accounts for the frequency dependence of traveltime measurements. From a data perspective, this means that traveltime measurements may in fact be made in multiple frequency bands in order to capture the dispersive nature of waves travelling through heterogeneous and dissipative media. While being beyond the scope of this study, such multiple-frequency measurements may greatly enlarge the dataset, thereby improving tomographic resolution (Sigloch et al., 2008). From an inversion perspective, the computation of correct finite-frequency sensitivities helps to avoid inversion artifacts related to the approximation of sensitivities. For instance, the approximation by infinitely thin rays may in fact lead to unrealistically good resolution by virtue of the central slice theorem (Iyer and Hirahara, 1993). In this context, the non-zero width of finite-frequency kernels naturally limits resolution to what is physically possible by using waves with a certain frequency content.

The second main advantage, specifically in medical ultrasound, is the ability to produce 3D images based on 2D acquisition systems. Slice-by-slice acquisition devices have gained popularity due to their fast reconstructions. However, they often suffer from artifacts caused by the 2D approximations of inherently 3D wave phenomena (Lavarello and Oelze, 2009; Goncharsky et al., 2016; Wiskin et al., 2017; Sandhu et al., 2017). Our work demonstrates that finite-frequency tomography does not only offer the possibility to image out of plane, but also to better constrain acoustic parameters by coupling data from different slices. This is a fundamental improvement compared to the current approaches, in which pseudovolumetric images are built by stacking 2D coronal slices (Duric et al., 2005). By using more 
accurate physical modelling, our approach respects the finite-frequency nature of the data, thereby reducing artifacts caused by $2 \mathrm{D}$ projections. Furthermore, it avoids subjective choices related to the post-processing of reconstructed images. In this sense, this work makes the critical contribution of providing truly 3D reconstructions using slice-by-slice devices, which may be important to accurately locate tissue anomalies inside the breast volume.

A prerequisite of our method is access to calibration data that ensure sufficient waveform similarity between cross-correlated waves. A direct consequence of waveform similarity is the possibility to compute the Jacobian operator at any frequency analytically, without suffering from time consuming numerical wave propagation simulations. The resulting linear inverse problem is very attractive for clinical practice, where fast and accurate solutions are indispensable for the recurring experiments. The possibility to compute properties of the forward operator prior to any experiment reduces the time to solution significantly.

To further reduce computational cost, we develop a 1D parameterization that allows us to represent any finite-frequency sensitivity kernel, independent of the emitter-receiver configuration, with the same analytic function. It encodes the full Jacobian operator, and we compute its elements on the fly for the matrix-vector operations required in each iteration of the least-squares solver. Our approach is ideal for GPU implementation, and, being matrix-free, it extends very efficiently to large-scale $3 \mathrm{D}$ problems. The latter is probably the most interesting application for USCT. For instance, in the 3D examples shown here, the computing time for 64768 sensitivity kernels is in the order of magnitude of $10^{0}$ seconds. Our code is implemented in MATLAB and uses one GPU card (Nvidia GeForce GTX 1060 $6 \mathrm{~GB})$.

In addition to the tomographic method, we apply a resolution analysis based on PSFs estimations. This is useful for a comparative assessment of the spatial resolution and inter-parameter trade-offs that arise from different experimental setups. For a quantitative interpretation of our results, we require better descriptions of the observational and forward modelling uncertainties, which can only be obtained with further experimental studies with known ground truths.

\subsection{Outlook: amplitude measurements}

Despite being a promising imaging tool, finite-frequency traveltime tomography still reduces to a single data point the information contained in the observed signals. It therefore misses valuable information about the media that might be encoded in other parts of the signals. In an attempt to increase the amount of exploitable data, several extensions of finite-frequency approach have been suggested in seismology. These include, for instance, finite-frequency tomography for amplitude measurements (Dahlen and Baig, 2002) and multiple frequency bands (Sigloch et al., 2008). In the following, we present some preliminary 


\section{Finite-frequency tomography}

results for amplitude tomography.

Studies using amplitude information to infer tissue velocity structure are not common. These mostly focus on retrieving tissue attenuation, but pressure wave amplitudes are also affected by velocity variations, which cause geometrical focusing and defocusing as well as diffraction and interference effects (Dahlen and Baig, 2002). In this section, we discuss the sensitivity of amplitudes to velocity variations.

\subsubsection{Theory}

Similar to traveltime tomography, we can formulate the finite-frequency amplitude tomography as a least-squares problem, where we minimize the sum of the squared relative residuals

$$
\frac{\delta A}{A}=\delta(\ln A)=\frac{A_{\mathrm{obs}}\left(\mathbf{x}_{r}, \mathbf{x}_{s}\right)-A\left(\mathbf{x}_{r}, \mathbf{x}_{s} ; \mathbf{m}\right)}{A\left(\mathbf{x}_{r}, \mathbf{x}_{s} ; \mathbf{m}\right)}
$$

between the observed and predicted amplitudes for each emitter-receiver combination, respectively. Here, we closely follow (Nolet, 2008) and define amplitude $A$ as the rootmean-square average over the pulse in the time interval $\left[t_{0}, t_{1}\right]$ :

$$
A\left(\mathbf{x}_{r}, \mathbf{x}_{s}\right)=\sqrt{\frac{1}{t_{1}-t_{0}} \int_{t_{0}}^{t_{1}} p^{2}\left(\mathbf{x}_{r}, t ; \mathbf{x}_{s}\right) d t}
$$

We again assume that the true velocity distribution can be expressed in terms of a homogeneous background velocity $c_{0}$ as $c(\mathbf{x})=c_{0}+\delta c(\mathbf{x})$, where the observed wavefield becomes $p_{\text {obs }}=p_{0}+\delta p$ whenever $\delta c(\mathbf{x})$ is sufficiently small. Upon inserting this into Equation (5.23), we obtain, to first order,

$$
\delta(\ln A)=\frac{\sqrt{\int_{t_{0}}^{t_{1}}\left[p_{0}+\delta p\right]^{2} d t-\int_{t_{0}}^{t_{1}} p_{0}^{2} d t}}{\sqrt{\int_{t_{0}}^{t_{1}} p_{0}^{2} d t}} \approx \frac{\sqrt{\int_{t_{0}}^{t_{1}}\left(p_{0}+2 p_{0} \delta p\right) d t}-\sqrt{\int_{t_{0}}^{t_{1}} p_{0}^{2} d t}}{\sqrt{\int_{t_{0}}^{t_{1}} p_{0}^{2} d t}}
$$

which we approximate using Taylor expansion as

$$
\delta(\ln A) \approx \frac{\int_{t_{0}}^{t_{1}} p_{0} \delta p d t}{\int_{t_{0}}^{t_{1}} p_{0}^{2} d t} .
$$

Because $\delta p=\frac{\delta p}{\delta c} \delta c$, Equation (5.26) defines the forward problem of the finite-frequency amplitude tomography, which has been already linearized. We can follow similar derivations as in Section 5.1 to obtain its explicit expression and identify the corresponding sensitivity kernel. For simplicity, we continue in the temporal Fourier domain, and similar 
to Equation (5.18), we transform Equation (5.26) to

$$
\delta(\ln A)=\frac{\operatorname{Re} \int_{0}^{\infty} p_{0}(\omega)^{*} \delta p(\omega) d \omega}{\int_{0}^{\infty} \omega^{2} p_{0}(\omega)^{*} p_{0}(\omega) d \omega} .
$$

Now, we use analytic 3D Green's function for homogeneous media [see Equation (5.21)], and the expressions for $p_{0}$ and $\delta p$ in Equations (5.19) and (5.20), to finally obtain

$$
\delta(\ln A)=\int_{V} K_{0}^{A}(\mathbf{x}) \delta c(\mathbf{x}) d \mathbf{x}
$$

with the sensitivity kernel

$$
K_{0}^{A}(\mathbf{x})=B(\mathbf{x}) \frac{\int_{0}^{\omega} \omega^{2}|f(\omega)|^{2} \cos \left(\frac{\omega}{c_{0}}\left(R_{x s}+R_{x r}-R_{s r}\right)\right) d \omega}{\int_{0}^{\omega}|f(\omega)|^{2} d \omega} .
$$

The comparison with the traveltime sensitivity kernel in Equation (5.22) reveals an almost equal structure. The main differences between both kernels is due to the sine instead of cosine function, which arises from the time derivatives in the pressure wavefield $p$. This suggests that, for finite-frequency amplitude tomography, we can reuse the same implementation as before, requiring minimal modifications.

Although the differences may seem minor, the impact is quite substantial. Figure 5.11 shows examples of sensitivity kernels for amplitude and traveltime measurements using a band-limited signal with frequencies in the range of 1-3 MHz. In both cases, we also illustrate the analytical pattern parameterized in terms of $R=R_{x s}+R_{x r}-R_{s r}$. As a result of the cosinusoidal term, the values in the amplitude sensitivity pattern appear phase shifted with respect to the traveltime ones, and the amplitude sensitivity exhibits its maximum along the geometrical ray path. Furthermore, as Dahlen and Baig (2002) observed, the values in the second Fresnel volume are particularly strong [see Figure 5.11(b)]. Using a paraxial approximation, they demonstrated that the cross-sectional area of the sensitivity its approximately zero. This means that any velocity anomaly that is constant across the width of the kernel will cause a negligible amplitude perturbation, and consequently, amplitude measurements are more sensitive to small-scale velocity variation (Fichtner, 2010). 


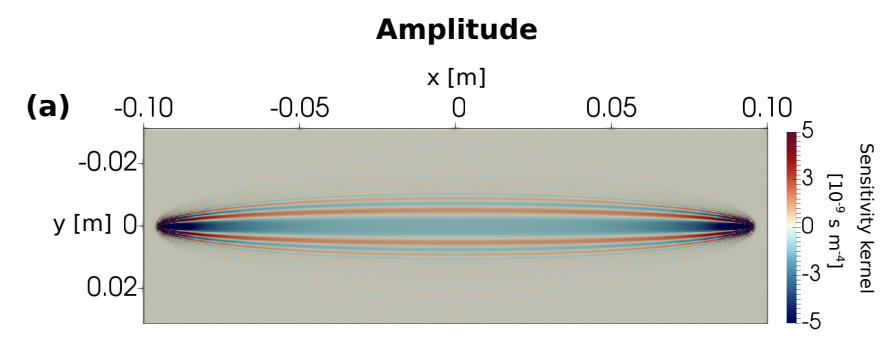

(b)

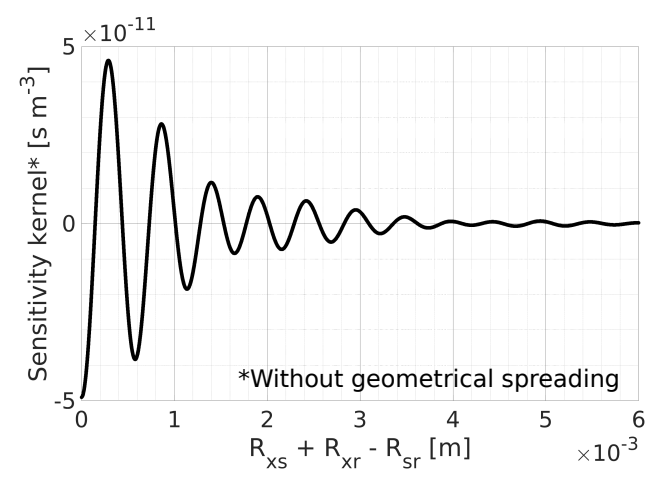

Traveltime

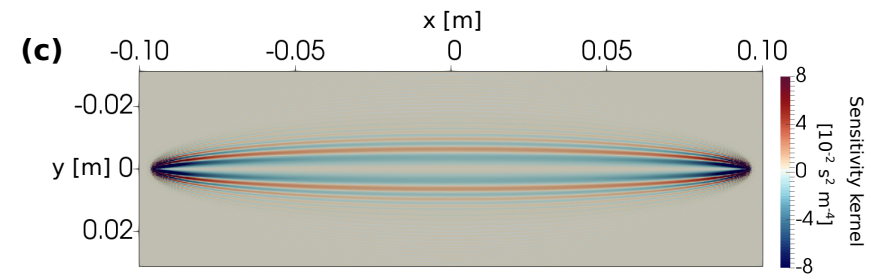

(d)

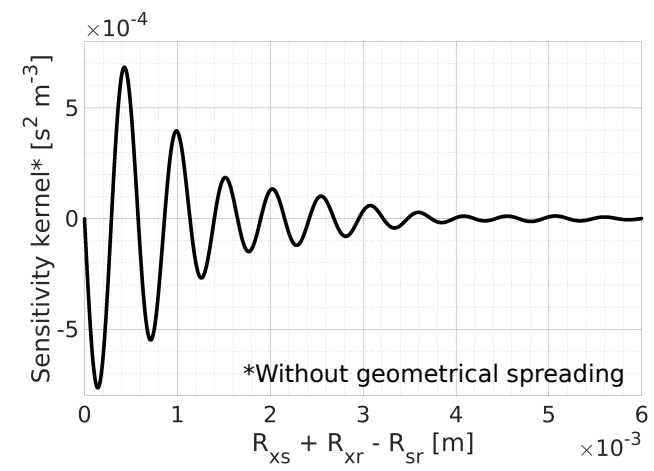

Figure 5.11.: (a) Sensitivity kernel for amplitude measurements for a band-limited signal with frequencies in the range of $1-3 \mathrm{MHz}$. The emitter and the receiver are located at positions $(-0.095,0,0) \mathrm{m}$ and $(0.095,0,0) \mathrm{m}$, respectively. (b) Sensitivity kernel in (a) represented as a function of $R=R_{x s}+R_{x r}-R_{s r}$, without the geometrical spreading term $B(\mathbf{x})$. (c), (d) Same as in (a) and (b) for traveltime measurements. 


\subsubsection{Numerical results}

We test the performance of the finite-frequency amplitude tomography in 2D numerical data. These were generated using the spectral element solver Salvus (Afanasiev et al., 2019) in a similar acquisition system as before, shown in Figure 5.3, but considering only half of the emitters. In our test, the true velocity model was only revealed once the reconstruction was obtained, and in this subsection we will proceed in this way. Later, however, this dataset has been part of the SPIE USCT Data Challenge 2019, where the ground truth was known for calibration purposes ${ }^{4}$.

In a blind test, the best strategy is to apply different imaging techniques to understand the nature of the phantom and build some prior information that can be included in the choice of the regularization. In particular, we use the B-mode technique to obtain high-resolution images of tissue reflectivity, shown in Figure 5.12(a). We observe that the phantom consists of several heterogeneities with circular and elliptical shape, and different size, including point-localized scatterers and anomalies that are smaller than the width of the first Fresnel zone $(9.4 \mathrm{~mm})$. Following this, we apply finite-frequency amplitude tomography using both second-order Tikhonov and TV regularization, and the results are shown in Figures 5.12(b) and 5.12(c), respectively. Although the scatterers are hard to identify in the reconstructions, our results demonstrate the potential of amplitude tomography to image small-scale structures that go beyond the width of the first Fresnel zone. Moreover, the size of the phantom appears to be consistent with the one observed in the reflectivity image. However, we also see some limitations: (1) strong artifacts are observed in the skin, which may be due to the choice of the regularization term; (2) the shape of the small heterogeneities appear elongated in the reconstructions; and (3) the absolute values of the velocity seem to be too low for a realistic breast tissue. To verify the latter, we reveal the true velocity model, shown in Figure 5.13. Effectively, our tomographic approach does not recover accurately the absolute value of the velocities. This may indicate some limitations in the linearity assumptions of the amplitude-velocity relationship. Although these are preliminary results, and therefore, do not represents an exhaustive study, our results indicate that amplitude measurements contain valuable structural information about acoustic tissue properties.

\footnotetext{
${ }^{4}$ Dataset $2 D$ ring aperture with known ground truth in https://usct.gitlab.io/datachallenge2019/ data/
} 

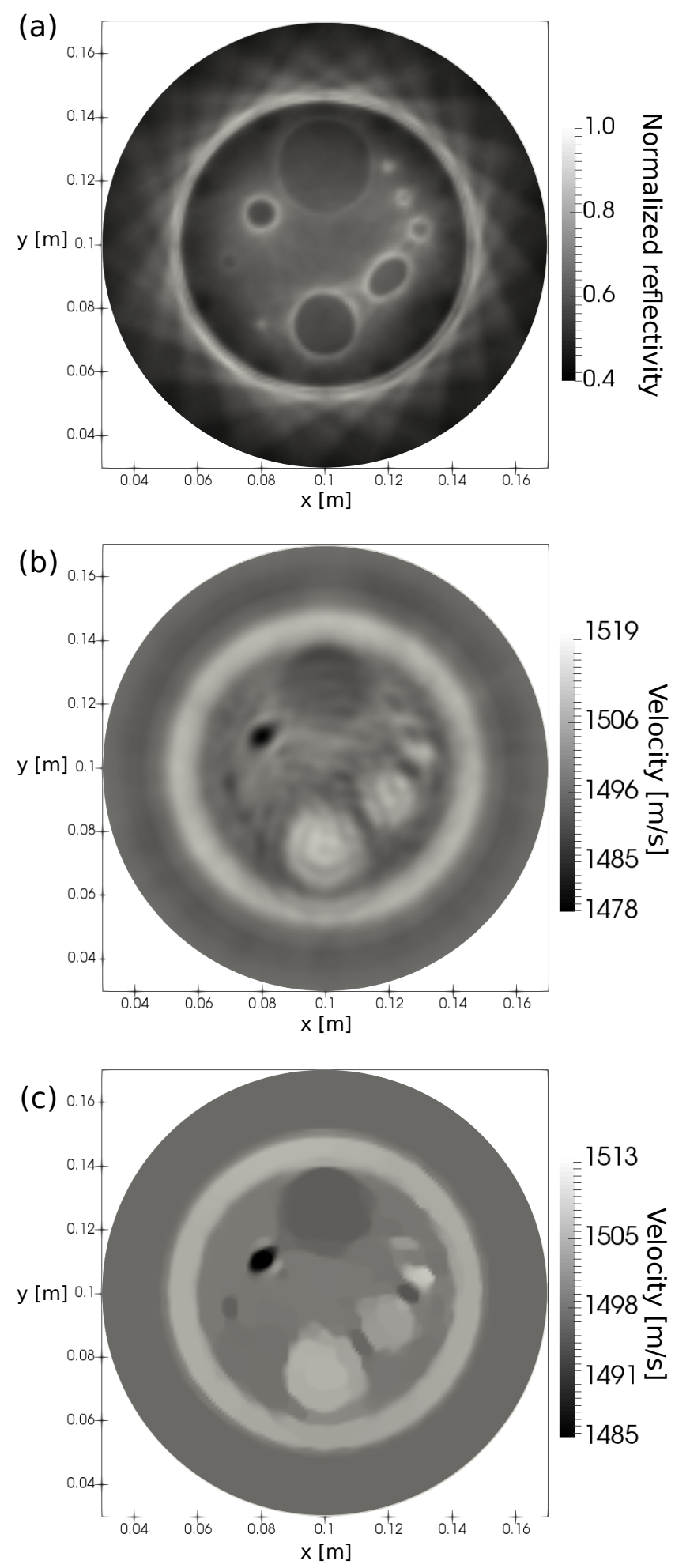

Figure 5.12.: (a) B-mode reflectivity image with normalized values. (b), (c) Velocity reconstruction for finite-frequency amplitude tomography, using second-order Tikhonov and TV regularization, respectively. 


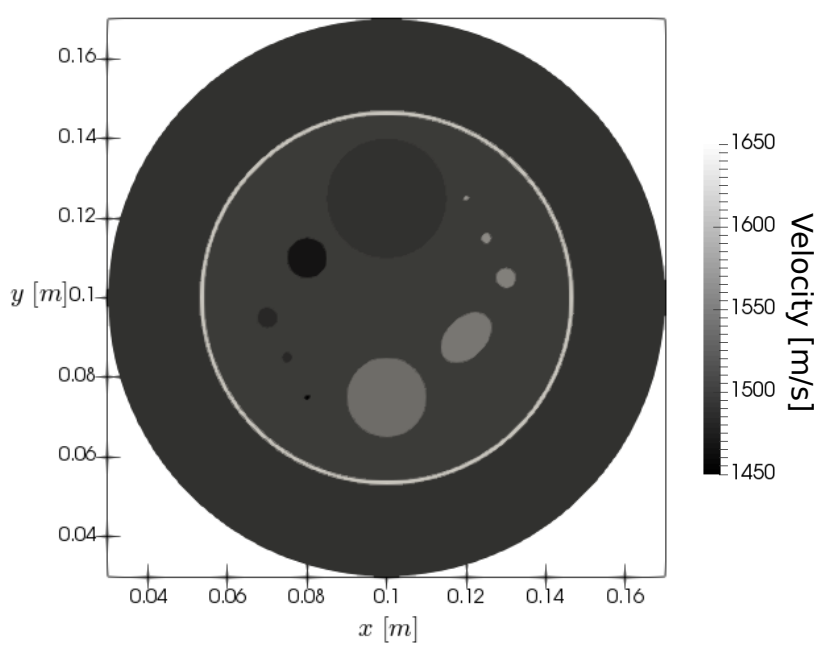

Figure 5.13.: True velocity phantom used to generate the dataset.

\subsubsection{Lab data application}

To understand if amplitude measurements are reliable in real data application, we reconstruct the 2D velocity distribution corresponding to the dataset in Section 5.2. Figure 5.14 displays the result obtained using only amplitude information, i.e., we do not consider any joint reconstruction using traveltime and amplitude measurements, although this would be our ultimate purpose. In this example, our goal is to understand the kind of information that amplitude measurements contain and compare this with traveltime measurements. Our result demonstrates that indeed amplitude measurements can be useful to better constrain certain properties of the model. These specially include the shape and the size of the breast, which often show artifacts when only traveltime information is used. Again, our result shows previously observed limitations of the method in retrieving the absolute values of the velocities, which are lower than the ones obtained in Section 5.2. Yet, velocity reconstructions using only amplitude measurements are very rare, which makes our result quite unique and therefore interesting from a scientific point of view.

\subsubsection{Conclusion}

Amplitude measurements contain valuable structural information that can be useful to better constrain the model parameters using joint reconstruction approaches together with traveltime measurements. In reality, however, amplitudes of the ultrasonic signals are also affected by attenuative effects, which are difficult to disentangle from purely acoustic ones. For a comprehensive integration of amplitude measurements in the tomographic inversions, tissue attenuation must be included as parameter to infer. This will require an extension of the finite-frequency tomography that considers amplitude sensitivities to 


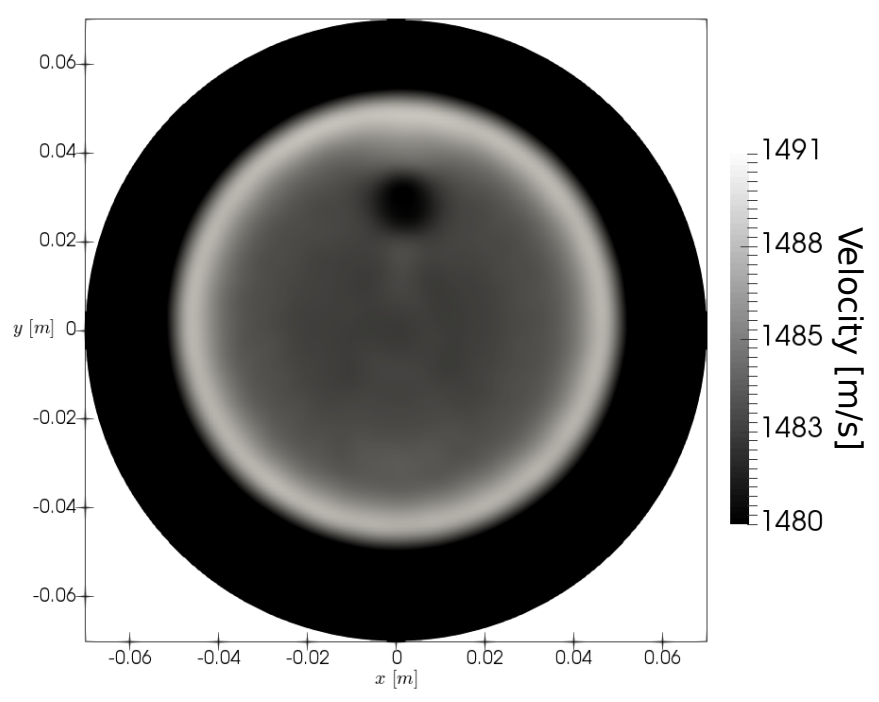

Figure 5.14.: 2D velocity reconstruction using finite-frequency amplitude tomography using the dataset from Section 5.2.

attenuation and performs multiple-parameter inversions (e.g., Sigloch and Nolet, 2006). We can compute these sensitivities similar to the amplitude kernels for velocity, by recognizing that attenuation effects can be modelled using imaginary components of the velocities (e.g., Nolet, 2008). 


\section{Designing USCT experiments: Part $I^{\ddagger}$}

Developing sophisticated tomographic methods is essential to maximize the amount of exploitable information that is contained in the observed measurements. However, there is no imaging method that can compensate for the missed information caused by a poorly designed experiment. One cannot extract what data do not contain. Carefully designed acquisition systems are therefore crucial for the success of any medical imaging technology. A satisfactory experiment will guarantee the acquisition of the most informative data about our region of interest, while controlling other aspects that constrain the amount of data collected in clinical applications. These include, for example, the data acquisition and processing time, and the computational cost of the image reconstruction. Consequently, one wishes to provide an effective experiment that responds best to the compromise or trade-off between both quality and cost.

Despite their importance, methods to optimally design the experiments usually receive very little attention in the literature. Intuitive or heuristic approaches are often more common that either (1) try to compensate the insufficient or inadequate data by increasing the amount of acquired data as much as possible, or (2) choose configurations that maintain spatially regular distribution of emitters and receivers on the device. The first approach may include potentially redundant information, thus unnecessarily increasing the acquisition and computational time. Regarding the second approach, several studies have demonstrated that indeed non-uniform designs provide superior results in terms of parameter resolution (e.g., Gemmeke et al., 2014; Curtis, 2004a). This is due to unequally balanced data coverage that uniform designs tend to produce.

The following two chapters introduce Optimal Experimental Design (OED) methods to USCT. They offer a systematic and quantitative tool to assess the quality of different designs and optimize them with respect to some predefined design parameters. We divide the study on OED methods in two parts. This chapter represents the first part and is particularly focused on exploring acquisition systems that are optimized for transmission imaging. We refer to this as individual optimization. The next chapter, on the other hand, aims at jointly optimizing acquisition setups for both reflection and transmission reconstructions, and we refer to this approach as joint optimization.

${ }^{\ddagger}$ This chapter has been modified from: N. Korta Martiartu, C. Boehm, N. Vinard, I. Jovanović Balic and A. Fichtner (2017). Optimal experimental design to position transducers in ultrasound breast imaging. Proceedings SPIE 10139, Medical Imaging 2017: Ultrasonic Imaging and Tomography 


\section{Designing USCT experiments: Part I}

For individual optimizations studied here, we mostly consider traveltime tomography using straight rays, and we analyze to what extend the results from traveltime inversion can be useful for time-domain waveform tomography for imaging the velocity distribution $^{5}$. As mentioned before, providing cost-effective 3D acquisition systems still remains a challenge in USCT. Therefore, we specifically address the following question: what are the locations of the transducers in a $3 \mathrm{D}$ experimental setup that maximize the transmission information contained in the measured ultrasound data? How does the

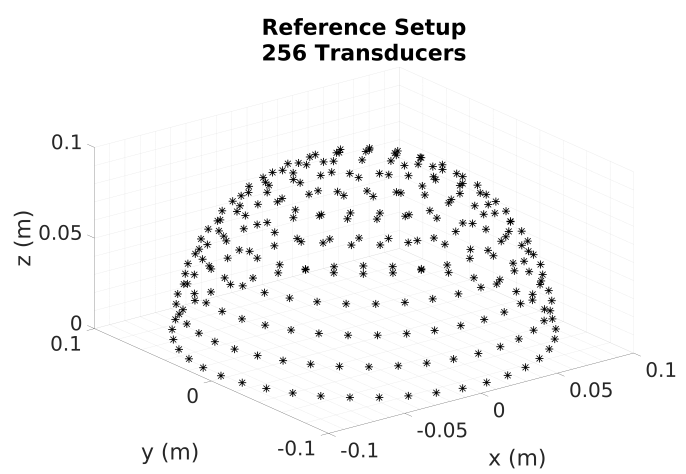

Figure 6.1.: Reference setup with regularly distributed 256 transducers in the surface of a hemispherical device of radius $0.1 \mathrm{~m}$. resulting experimental design differ from a reference setup consisting of regularly distributed transducer locations [see Figure 6.1]? Addressing these questions will allow us to describe the theory and general formulation of OED methods, useful also for the next Chapter 7. We first discuss several definitions of the quality measure, which quantifies the level of expected uncertainties in the estimated parameters, and then we provide different approaches to solve the optimization problem. The latter can be addressed either by global optimizers or sequential algorithms. We conclude the present chapter by comparing both approaches using numerical examples that optimize the reference setup in Figure 6.1. To ultimately provide a cost-effective 3D acquisition system, this setup combines characteristics of other USCT devices. Here, the number of transducers is 256, same as in the CURE system Duric et al. (2007), while the aperture shape is similar to the KIT 3D USCT system Gemmeke et al. (2017).

\subsection{Optimal experimental design}

The overall goal of OED methods is to maximize the information content of observed measurement, by minimizing the expected post-acquisition uncertainties in the estimated parameters describing the medium. Certainly, these uncertainties are related to specific image reconstruction methods, i.e., the forward operator $\mathbf{F}$, and quantified by the posterior covariance matrix $\boldsymbol{\Gamma}_{\text {post }}$. In Chapter 4 we observed that, in the context of Bayesian inference, where the estimated model parameters are interpreted in terms of probability density functions, the inverse of the Hessian $\mathbf{H}$ can be identified as the posterior covariance $\boldsymbol{\Gamma}_{\text {post }}$. While this is exact only for linear forward operators, the same strategy can be used for nonlinear operators to approximate the uncertainties in the vicinity of the global minimum of

\footnotetext{
${ }^{5}$ This study was done previous to developing the finite-frequency traveltime tomography approach.
} 
the objective function $\chi(\mathbf{m})$ [see Section 4.3]. In particular, the data-misfit Hessian $\mathbf{H}_{\text {misfit }}$ shows useful properties to measure the quality of an experimental configuration, mostly due to its dependency on the experimental design parameters $\mathbf{s}$. This allows us to compare different candidates and select the ones that give the lowest uncertainties in the parameter estimation. For linear problems, moreover, $\mathbf{H}_{\text {misfit }}=\mathbf{F}^{\prime}(\mathbf{s})^{T} \boldsymbol{\Gamma}_{\text {noise }}^{-1} \mathbf{F}^{\prime}(\mathbf{s})$ has additional two advantages: (1) it neither depends on the observations nor the unknown model parameters, which means that it could be used to optimize the experiment before any realization; and (2) it shows the same structure for any forward operator, under the condition that these are linear or can be linearized with respect to the model parameters. This allows us to present general formulations of the OED problem, independent of the tomographic method that is intended to be applied post-acquisition.

\subsubsection{Quality measure}

Our ability to invert the data-misfit Hessian $\mathbf{H}_{\text {misfit }}$ will determine how the uncertainties in the data space are mapped into the model parameters. For example, small eigenvalues of the matrix will yield large variances in the model parameters, which translates to higher uncertainty in the reconstruction. Assume that $\Theta$ is a scalar function that quantifies the level of expected uncertainties in the parameters by extracting some properties from $\mathbf{H}_{\text {misfit }}$. Then, we can formulate the OED problem as a minimization problem

$$
\min _{\mathbf{s} \in \mathcal{S}} \Theta\left(\mathbf{H}_{\text {misfit }}(\mathbf{s})\right),
$$

where $\mathcal{S}$ represents the space for all possible experimental configurations.

There are various definitions for $\Theta$ that have been successfully applied in the field of OED (Curtis, 2004a; Khodja et al., 2010; Maurer et al., 2010). The use of different design criteria mostly depends on the involved computational complexity for the specific problem under consideration. For example, one ideally would wish to compute each individual eigenvalue of $\mathbf{H}_{\text {misfit }}$, but this becomes intractable for large-scale problems. Here, we use the following quality measure:

$$
\Theta=-\sum_{i=1}^{N} \frac{\lambda_{i}}{\lambda_{1}}=-\frac{1}{\lambda_{1}} \operatorname{trace}\left(\mathbf{H}_{\text {misfit }}\right)
$$

where the eigenvalues $\lambda_{i}$ are sorted in order of decreasing magnitude, with $\lambda_{1}$ being the largest. Here we drop the dependency on $\mathbf{s}$ for clarity. A quality criterion involving the trace of $\mathbf{H}_{\text {misfit }}$ is easy to access and it only requires the diagonal entries of the matrix. We can further alleviate the cost of computing $\Theta$ by reducing the domain to a subset of parameters. This is particularly helpful to maximize the information about a specific ROI. Note that we can either minimize $\Theta$ or equivalently maximize $-\Theta$, which is more natural for this particular quality criterion. However, we believe that is more intuitive to 


\section{Designing USCT experiments: Part I}

formulate Equation (6.1) as a minimization problem, and also more convenient for other quality measures that we will introduce in Chapter 7 .

The quality measure in Equation (6.2) is normalized by the largest eigenvalue to avoid selecting configurations with only a few very large eigenvalues. This ensures that Equation (6.2) cannot be minimized by only increasing the information within a small part of the domain at the expense of a poor coverage of the rest. An example of this is illustrated in Figure 6.2, where we use an intuitive toy problem with four parameters and four transducers, and we consider traveltime tomography using straight rays. We optimize the experimental design by minimizing the quality measure in Equation (6.2) with and without normalizing by the largest eigenvalue, shown in Figures 6.2(b) and 6.2(a), respectively. As we observe, when the normalization factor is not considered, the largest eigenvalue dominates the trace, and by maximizing this, we find an experimental design that is just informative about a subdomain of our ROI. In this toy example, the subdomain equals to one model parameter. Figure 6.2(c) shows the corresponding normalized eigenvalue spectra for its case. Unlike the trace, the setup obtained by the normalized trace ensures significant information in all eigenvalues, ultimately improving the spatial resolution of the reconstruction.

\begin{tabular}{|l|c|c|c|c|}
\cline { 2 - 5 } \multicolumn{1}{c|}{} & \multicolumn{2}{c|}{ Traveltime inversion } & \multicolumn{2}{c|}{ Waveform inversion } \\
\cline { 2 - 5 } \multicolumn{1}{c|}{} & trace & norm. trace & trace & norm. trace \\
\hline Experimental Design Figure 6.2(a) & 12 & 1 & 8.0643 & 1.0026 \\
\hline Experimental Design Figure 6.2(b) & 9 & 3 & 14.3615 & 3.5213 \\
\hline
\end{tabular}

Table 6.1.: Absolute and normalized values of the trace of $\mathbf{H}_{\text {misfit }}$ for experimental designs shown in Figure 6.2. These are computed using both traveltime and waveform tomography.

\subsubsection{Comments on non-linear inverse problems}

For non-linear inverse problems, the data-misfit Hessian depends on both the design and model parameters. Due to relatively small variations in the velocity of breast tissue, however, we do not expect significant changes in the spectrum of $\mathbf{H}_{\text {misfit }}$ for different model parameters. This enables us to neglect the dependency on the model and to use the same quality measure for waveform inversion. Figure 6.2(d) shows the corresponding normalized eigenvalue spectra of the Hessian operator for both experimental designs found in Figures 6.2(a) and 6.2(b). When comparing with Figure 6.2(c), similarities in both spectra suggest that traveltime inversion could provide a computationally tractable approximation to optimize the experimental design for waveform inversion approach. In addition, Table 6.1 shows the corresponding absolute and normalized values of the trace of the Hessian for both tomographic approaches. In contrast to traveltime tomography, the configuration shown in Figure 6.2(b) provides higher values in both quality criteria when waveform 
(a)

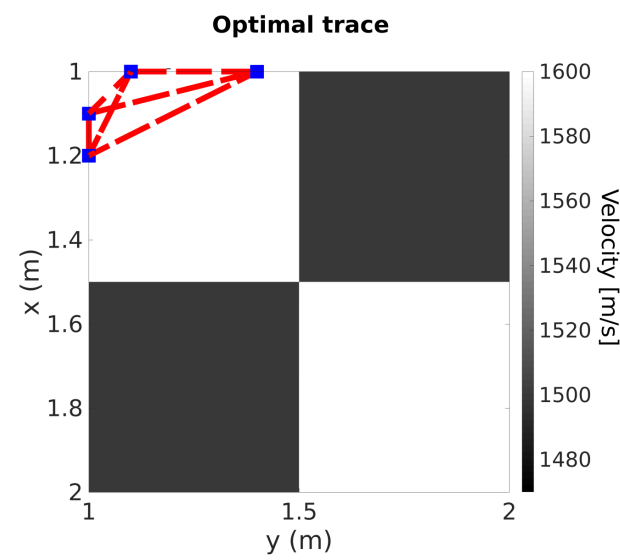

(c)

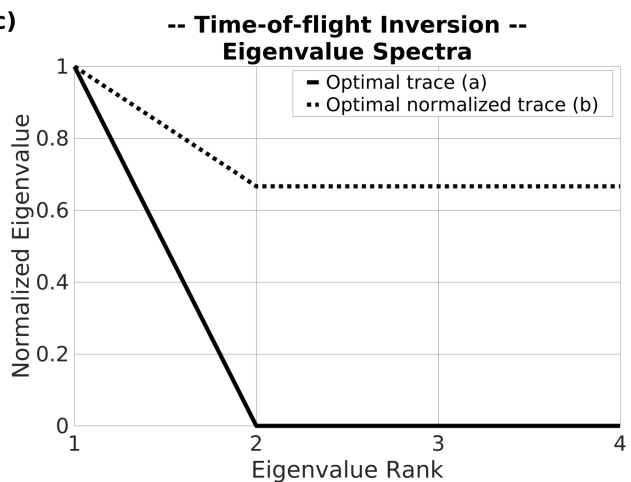

(b)

\section{Optimal normalized trace}
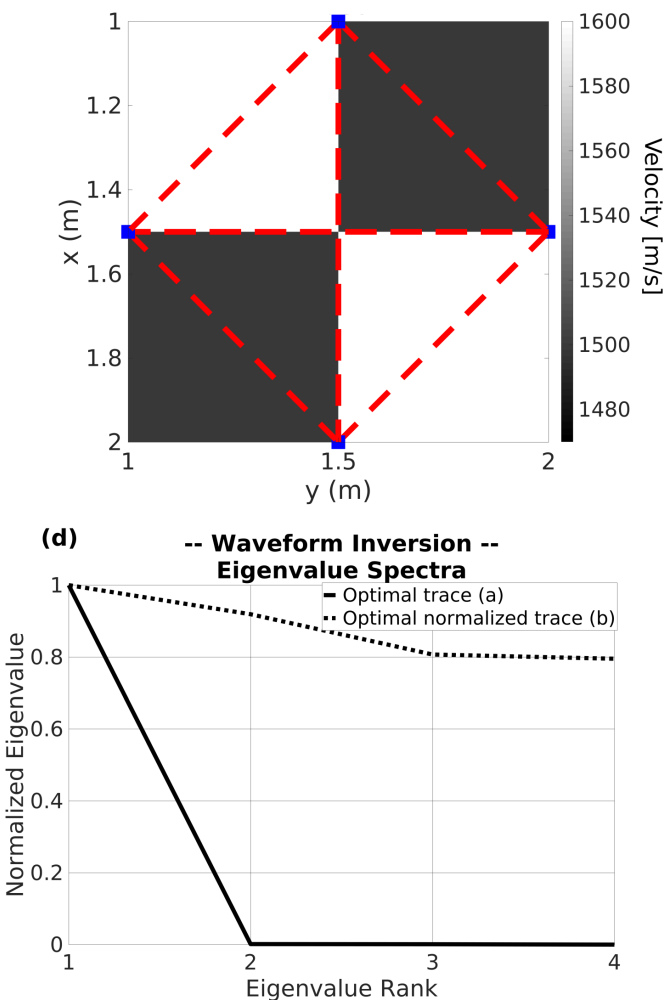

Figure 6.2.: Optimized transducer locations considering traveltime inversion that maximizes (a) the trace of $\mathbf{H}_{\text {misfit }}$ and (b) the normalized trace of $\mathbf{H}_{\text {misfit }}$ by its largest eigenvalue. Blue squares indicate the transducers, and red dashed lines show the corresponding straight-ray paths. (c), (d) Normalized eigenvalue spectra of $\mathbf{H}_{\text {misfit }}$ for traveltime and waveform inversion, respectively. In both cases, we compare the spectra corresponding to the solutions in (a) and (b).

tomography is considered. Therefore, to avoid numerical errors in the approximation of the largest eigenvalue, waveform inversion could benefit from the trace without normalization as the quality measure.

Properties of the Hessian can be estimated using matrix-free methods (Alexanderian et al., 2016). In particular, adjoint techniques allow us to compute Hessian-vector products at the cost of four wave propagation simulations per vector (Fichtner and Trampert, 2011a; Boehm and Ulbrich, 2015). For large-scale problems, however, estimating the trace of $\mathbf{H}_{\text {misfit }}$ can be computationally prohibitive, and stochastic trace estimators have been successfully applied as alternative (Haber et al., 2008; Alexanderian et al., 2016). They only require few Hessian-vector products, becoming therefore relatively efficient. In this study, we consider a variation of the trace estimation method proposed by Hutchinson (Hutchinson, 1990; 


\section{Designing USCT experiments: Part I}

Avron and Toledo, 2011), i.e.,

$$
\operatorname{trace}\left(\mathbf{H}_{\text {misfit }}\right) \approx \frac{n}{N} \sum_{i=1}^{N} \frac{\mathbf{v}_{\mathbf{i}}^{T} \mathbf{H}_{\text {misfit }} \mathbf{v}_{\mathbf{i}}}{\mathbf{v}_{\mathbf{i}}^{T} \mathbf{v}_{\mathbf{i}}},
$$

where $\mathbf{v}_{\mathbf{i}}$ are random vectors with values independently drawn from a normal distribution, and $N$ is the sample size of the estimator, i.e., the number of random vectors.

\subsubsection{Optimization strategies}

Our ultimate goal in OED is to optimize the measurements collected from USCT acquisition systems using Equation (6.1). The quality measure $\Theta$ depends on the design parameters $\mathbf{s}$ in a non-linear manner, and ideally, to solve the optimization problem, one should exhaustively sample the space of all possible experimental configurations $\mathcal{S}$. In geophysical applications, a variety of global optimization algorithms have been used for this purpose (Hardt and Scherbaum, 1994; Curtis, 1999a; Ajo-Franklin, 2009; Roux and Garcia, 2014). We refer to these approaches as Global Optimal Experimental Design (GOED) methods (Maurer et al., 2017). However, exploring the entire design space $\mathcal{S}$ can become prohibitively expensive for large-scale problems, and to make the optimization problem computationally tractable, Sequential Optimal Experimental Design (SOED) methods have been proposed as an alternative (Guest and Curtis, 2009; Maurer et al., 2010). In the following, we discuss in detail both approaches, and we provide for GOED efficient implementations that are focused on our particular problem.

\subsubsection{Global optimal experimental design}

In this study, we consider Genetic Algorithm (GA) (Chipperfield and Fleming, 1995) to solve Equation (6.1) with respect to the transducer locations. We refer the reader to Gallagher and Sambridge (1994) for a detailed description on GA. Although this algorithm does not ensure finding the globally optimal solution, we may use it to increase the quality measure of the reference setup and ensure an improvement in the posterior uncertainties of reconstructed images. Because GA is a stochastic optimization method, it may converge to different solutions in each run. We therefore perform the optimization several times and use the result with the overall highest quality value.

Our goal is to determine the optimal locations of 256 transducers on the surface of the hemispherical device shown in Figure 6.1. To optimize the transducer configuration, we describe an experimental design using a vector $\mathbf{s}$ with elements defining transducer locations in spherical coordinates. Due to the spherical symmetry of the scanning device, each position can be defined using two coordinates, the polar and azimuth angle. Therefore, $\mathbf{s}$ has a total of 512 elements. For such a large number of design parameters, sampling 
the space of all possible experimental configurations may be computationally infeasible. However, GA tends to preserve the information and structures contained in successful designs during the evolutionary process, and thus, we can speed up its performance by providing an initial guess relatively close to globally optimal solution.

To obtain a suitable starting point, we suggest to exploit the azimuthal symmetry of our problem and solve a set of 2D OED problems in sagittal and coronal directions. A 3D experimental design can then be constructed by combining the transducer locations resulting from the individual slices. Because GA samples the space of all possible experimental configurations, it only requires the forward problem to compute the quality of each design. Therefore, our approach not only reduces the subspace of designs that are sampled, but also reduces the computational cost of recurring evaluations of the forward problem, which may be particularly expensive for large-scale non-linear problems.

In the study presented here, we apply GOED method for traveltime tomography using straight rays. We then compare the quality measure of those configurations using waveform tomography. This helps us to analyze the extent to which traveltime tomography could provide a useful approximation for optimizing the experimental setup for waveform tomography. Although extending the method described above to waveform inversion is straightforward, it would require significantly more computational resources.

\subsubsection{Sequential optimal experimental design}

To further reduce the computational cost of GOED problems, other strategies can be applied. For instance, a common strategy is to predefine the total number $N_{s}$ of candidate locations for the transducers, and to define the experimental design $\mathbf{s}$ as an array of binary entries that selects a subset of them: 0 for nonactive transducers, and 1 for the active ones. Although this approach reduces significantly the design space $\mathcal{S}$, it still includes all transducer combinations, the total number being $\sum_{i=1}^{N_{s}}\left(\begin{array}{c}N_{s} \\ i\end{array}\right)=2^{N_{s}}-1$. This may therefore become very large for problems with a large number of design parameters $N_{s}$.

To make the optimization problem computationally tractable, we apply the SOED method. This is an iterative approach in which we deselect (or select) the most redundant (or the most informative) transducer at each iteration. The SOED algorithm does not necessarily guarantee a globally optimal solution for a fixed number of transducers. However, studies in seismic tomography comparing SOED with global optimization algorithms have shown that both approaches yield very similar solutions (Guest and Curtis, 2009). In this chapter, we will indeed compare the solutions obtained by both GOED and SOED approaches for the optimization of the 3D setup shown in Figure 6.1, and analyze the validity of SOED as a cost-effective alternative to GOED.

The SOED algorithm can be applied either in an iteratively destructing or constructing manner, which means that either we deactivate or activate a transducer location in 


\section{Designing USCT experiments: Part I}

each iteration. The Algorithm 1 provides an overall description of our implementation of the SOED method using the iteratively destructing approach. Here, we denote by $\mathbf{s}_{k}=\left(s_{1}, \ldots, s_{N_{s}}\right) \in\{0,1\}^{N_{s}}$ the transducer configuration found at the $k$-th iteration. Our method samples a total number of $\frac{N_{s}\left(N_{s}+1\right)}{2}$ experimental designs.

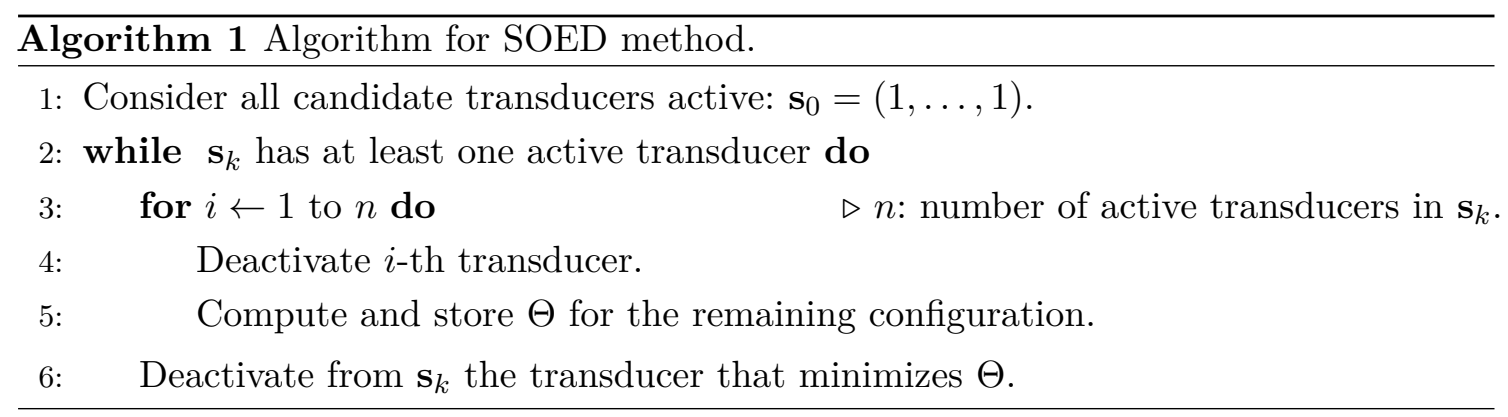

Due to the sequential nature of the algorithm, SOED has the advantage of providing benefit-cost curves. An example of this is shown in Figure 6.3. They illustrate the gain in the data information content with respect to the experimental cost (Maurer et al., 2017). They are particularly useful to maximize the benefit-cost ratio of the experiment by identifying redundancies in the observations. In the next Chapter 7, and in particular in Section 7.2, we discuss these curves in more detail using a toy example.

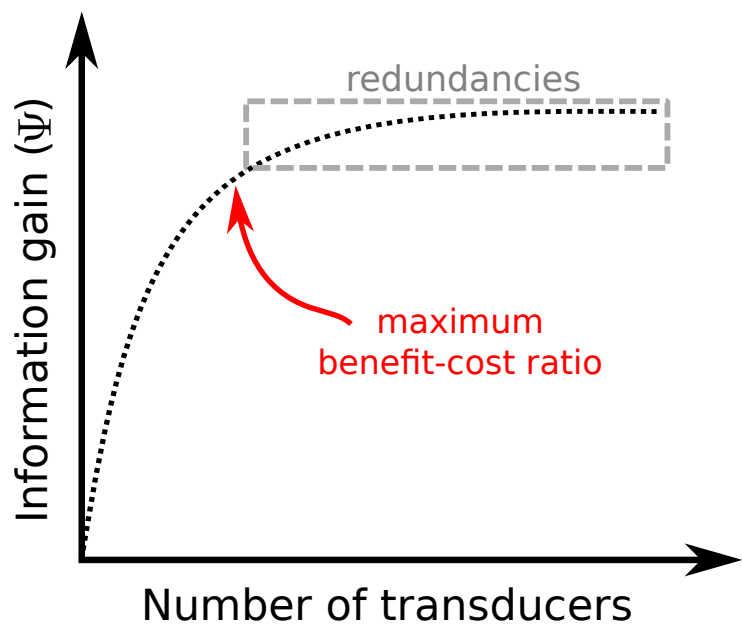

Figure 6.3.: Illustrative example of the benefit-cost curve obtained from SOED approach. 


\subsection{Numerical results: 3D hemispherical setup}

The reference experimental design considered in this study is shown in Figure 6.4(a). It consists of 256 transducers that are uniformly distributed on the surface of a hemisphere with a radius of $10 \mathrm{~cm}$. Here, every transducer acts in sending and receiving mode; that is, sequentially each transducer is emitting, while all other transducers are recording the signal. We discretize our domain using a rectilinear grid of $2 \mathrm{~mm}$ mesh size, and we consider the 3D breast phantom shown in Figures 6.4(b) and 6.4(c). These figures show a sagittal slice at $x=0 \mathrm{~m}$ and a coronal slice at $z=0.006 \mathrm{~m}$, respectively. Although for linear problems the quality measure does not depend on the model parameters, this will be useful to validate our results with velocity reconstructions. The numerical breast model is based on the so-called Derenzo phantom (Derenzo et al., 1981), which is commonly used in medical imaging to test the spatial resolution of reconstructed images. Because the temperature of the surrounding water can be controlled and, thus, its acoustic properties are known, the model space is restricted to the subregion that encloses the breast including a small threshold to account for an inaccurate positioning or patient movement. This defines the ROI and is indicated by red dashed lines in Figure 6.4. It allows us to eliminate all columns of $\mathbf{F}^{\prime}$ corresponding to model parameters $\mathbf{m}$ that are outside of this region and reduce the computational cost of the problem.

\subsubsection{Building a suitable initial configuration}

To obtain an adequate starting configuration for GA, we solve a set of 2D GOED problems using traveltime tomography. First, we optimize the locations of transducers in the sagittal direction. Here we consider 18 transducers, which corresponds to the total number of transducers defined in each slice of the reference experimental design [see Figure 6.4(a)]. Once the optimized transducer locations are determined in the sagittal direction, we consider 12 coronal slices corresponding to those elevations. Figure 6.5 shows the best configurations found by our GOED algorithm for sagittal and coronal slices indicated in Figure 6.4(a).

A first general conclusion may be drawn from the solution in the sagittal direction, shown in Figure 6.5(b). The optimal configuration gradually increases the spacing between the transducers from top to bottom. This compensates for the poor ray coverage in the upper part of the breast close to the chest wall, which is clearly observed in the uniform configuration [see Figure 6.5(a)]. This design feature is intuitively understandable and can be generalized easily to a 3D configuration. Furthermore, Figure 6.5(e) compares the corresponding normalized eigenvalue spectra for uniform and optimized designs. As expected, higher values in the optimized case indicate that the optimized setup reduces the posterior uncertainties in the estimated model parameters. In addition, it provides a larger number of significant eigenvalues, which indeed will result in better resolved velocity parameters. 


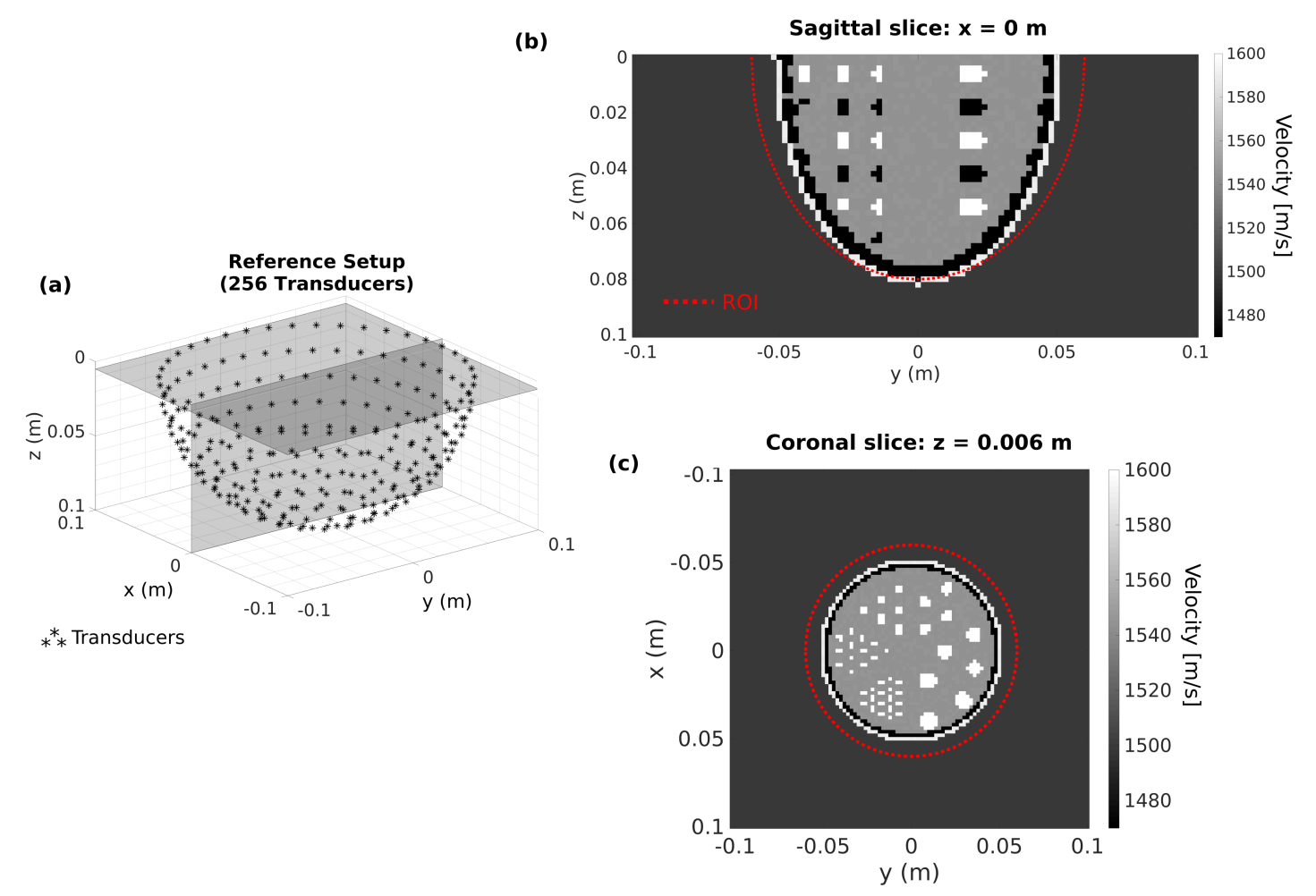

Figure 6.4.: (a) Reference setup with 256 transducers on the surface of a hemisphere with radius $10 \mathrm{~cm}$. (b) Sagittal slice at $x=0 \mathrm{~m}$ and (c) coronal slice at $z=0.006 \mathrm{~m}$ of the numerical breast model used for velocity reconstructions. This is referred as Derenzo phantom, and the location of the slices are indicated by gray planes in (a). The dashed lines next to the phantom indicates the ROI in which we evaluate the quality measure.

In the coronal plane, the optimized design suggests the need of breaking the symmetry of the uniform design in order to improve the ray coverage at the center of the ROI. This can be observed in Figures 6.5(c)-6.5(d), which show the uniform and optimized configurations at $z=0.006 \mathrm{~m}$, i.e., the upper part of the breast close to the chest wall. The eigenvalue spectra for both designs, shown in Figure 6.5(f), are very similar in this case. However, there are two important improvements: (1) the optimized configuration improves the magnitude of dominant eigenvalues in the normalized spectrum, and (2) the number of significant eigenvalues is increased, which can be seen for rank 220 - 257. Similar features can be observed for other horizontal slices at different elevations.

\subsubsection{Full 3D optimization}

Based on these results, we construct a 3D configuration by stacking together the individual slices. The middle row in Figure 6.6 shows the 3D design that is derived from 


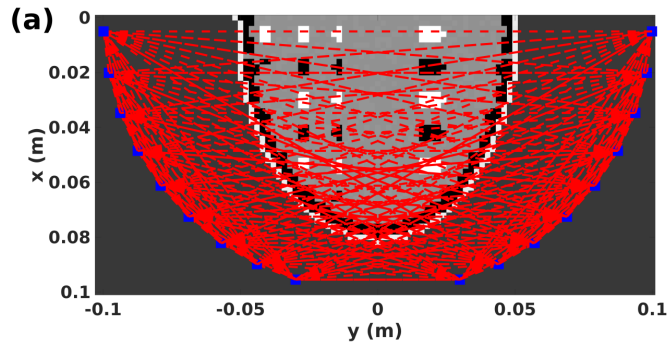

Uniform design

(c)

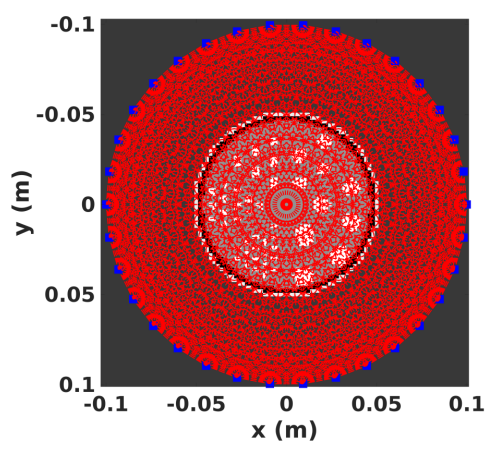

(e)

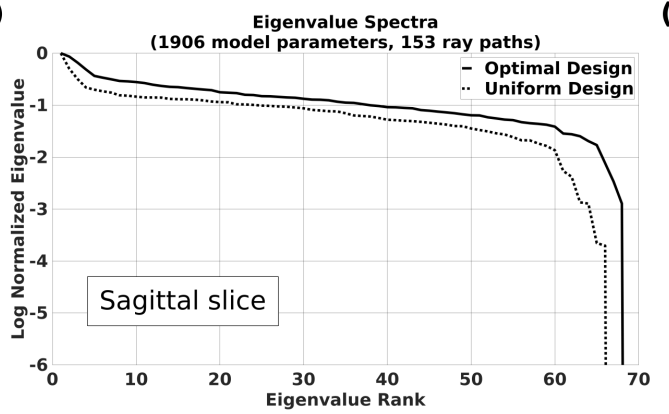

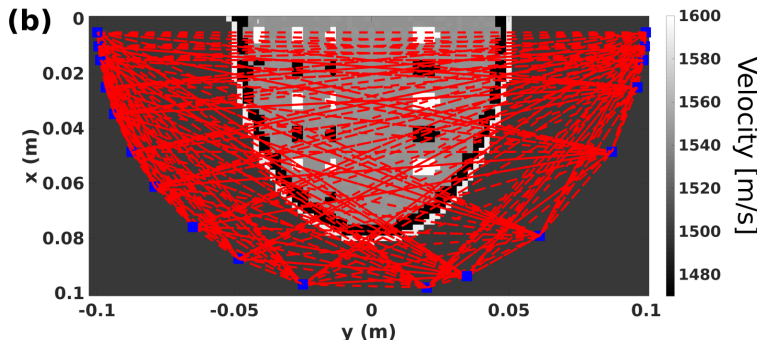

Optimal design

(d)

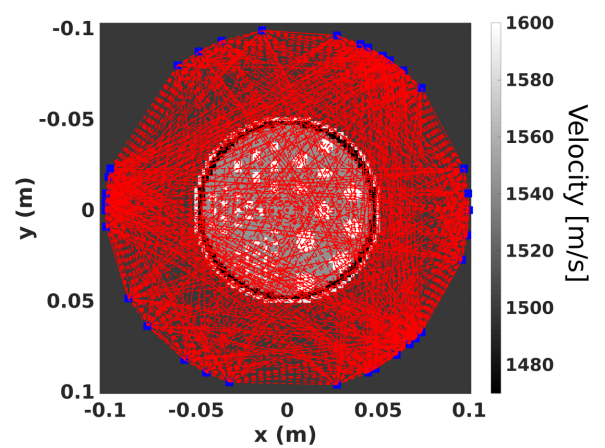

(f)

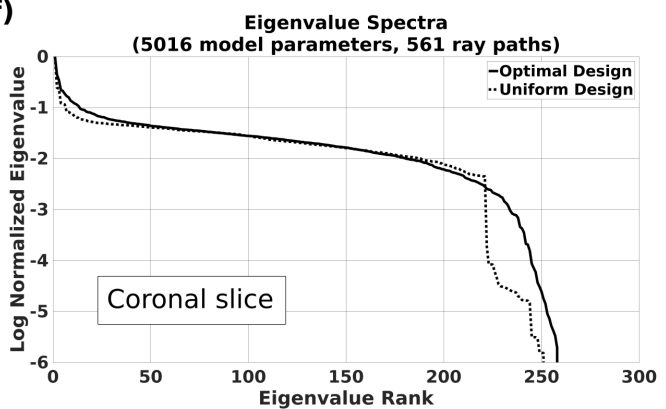

Figure 6.5.: (a) Uniformly distributed reference configuration using 18 equidistantly spaced transducers (blue) and corresponding straight ray paths (red lines) for a 2D sagittal slice at $x=0 \mathrm{~m}$. A velocity model of the breast has been included in the background for a better understanding of the experiment. (b) Optimized experimental design using Equation (6.2) for the sagittal plane. (c) Uniformly distributed reference configuration using 34 transducers in a coronal slice at $z=0.006 \mathrm{~m}$. (d) Optimized transducer locations for the coronal case. (e), (f) Comparison of normalized eigenvalue spectra between uniform and optimized designs in the sagittal and coronal plane, respectively.

the set of optimal solutions in the sagittal and coronal directions. The pattern we obtain significantly differs from the uniform configuration, shown in the top row of Figure 6.6. The new design is then used as initial guess for the full 3D global optimization, and after performing the GOED five times, we select the solution with the highest quality value. The bottom row of Figure 6.6 shows the resulting optimal 3D configuration of transducers. Clearly, the algorithm finds an interesting and initially non-intuitive pattern: most of the transducers are gathered in three specific regions of the hemisphere, showing approximately 


\section{Designing USCT experiments: Part I}

a rotational symmetry of order three $\left(120^{\circ}\right)$ with respect to the vertical axis. Furthermore, more transducers are located in the part of the hemisphere that is close to the chest wall, a feature that has been already observed in the $2 \mathrm{D}$ optimization in sagittal direction [see Figure 6.5(b)].

Figure 6.7 shows the comparison between eigenvalue spectra corresponding to the solutions in Figure 6.6. It certainly indicates the benefits of GOED and verifies the efficiency of our approach of constructing 3D configurations from 2D optimized slices. The optimal experimental design does not only improve the relative values of the eigenvalues but also increases the number of significant eigenvalues, which allows us to collect information about the model that is missing in the reference configuration. However, we also observe trade-offs when comparing the eigenvalue spectra of the optimal experimental design with the design constructed from the intermediate 2D results. In particular, a higher design quality value does not necessarily imply a higher number of significant eigenvalues. This corresponds to an increased resolution in some parts of the domain, at the expense of a poorer coverage in other parts.

To better understand the impact of the results, we compute velocity reconstructions using the optimized experimental designs and compare them with the reference configuration. Traveltime measurements are collected using 3 rotations of the device, each one with an angle of $40^{\circ}$, and we add Gaussian noise with a standard deviation of $0.2 \%$ to the traveltime observations. Furthermore, we apply the same regularization parameter in the inverse problem to give a fair comparison between the results of different configurations. Although rotations of the device are not considered in the GOED problem, they allow us to increase the number of measurements, preserving the relative positions between the transducers. Here, the angle and number of rotations is chosen according to the rotational symmetry of the optimized setups, but other choices are also possible.

Figure 6.8 shows vertical and horizontal slices of velocity reconstructions obtained from the experimental designs in Figure 6.6. The results clearly show how the optimized configuration improves the spatial resolution in the region close to the chest wall, which is indeed of special interest in breast cancer detection. The uniform design fails in collecting information within the mentioned region. Hence, the velocity reconstruction does not contain any fine-scale features and is merely the result of the regularization term. 


\section{(a) Reference setup}
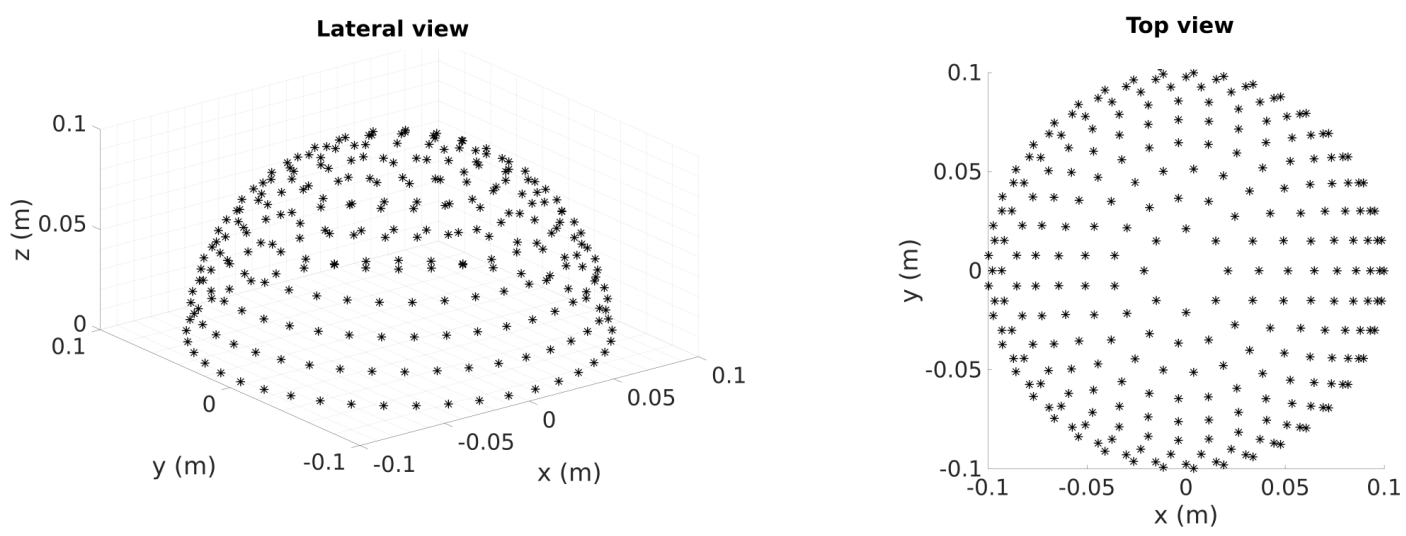

(b) Optimized setup from 2D OED
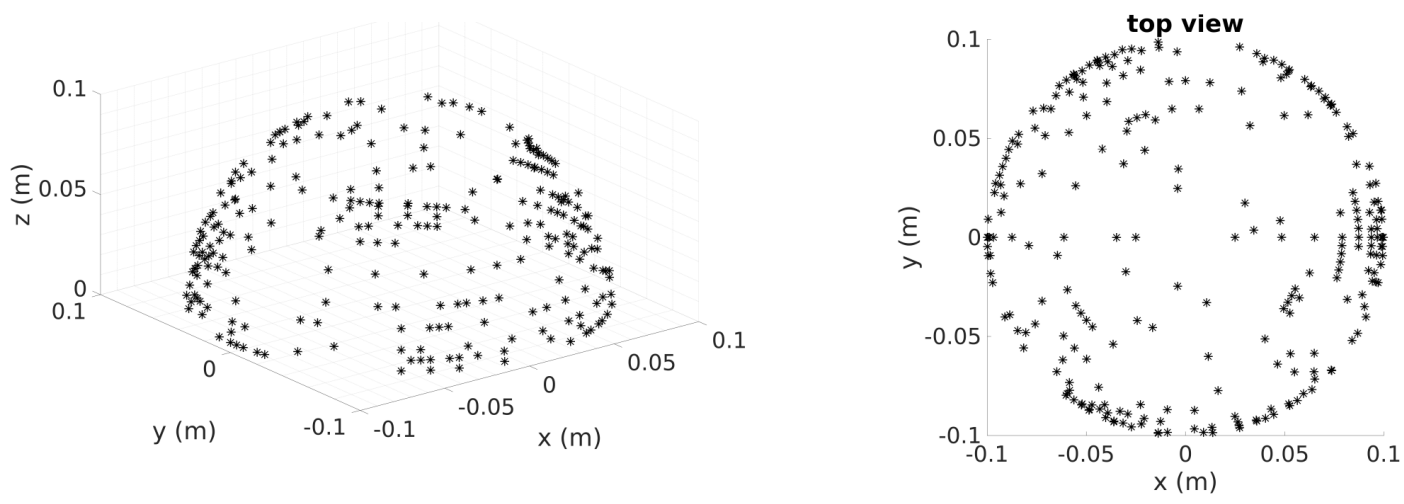

(c) Optimal setup 3D OED
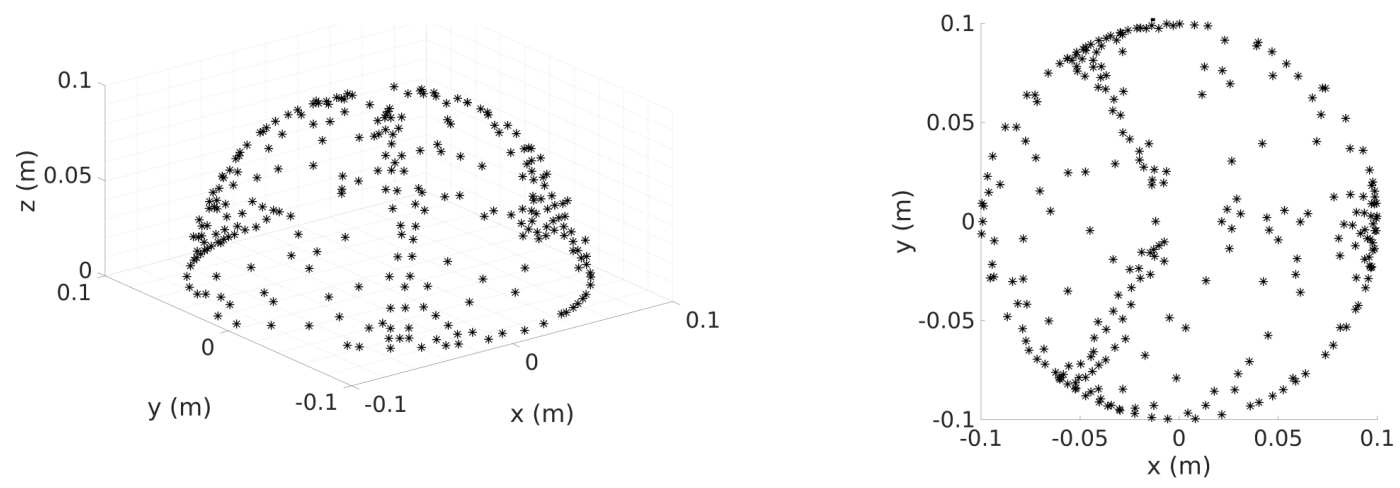

Figure 6.6.: (a) Top and lateral view of the reference experimental design with uniformly distributed transducers. (b) 3D experimental design built from 2D GOED results. (c) Optimal 3D experimental design obtained by applying GA to the solution in (b), used as initial guess. 


\section{Designing USCT experiments: Part I}

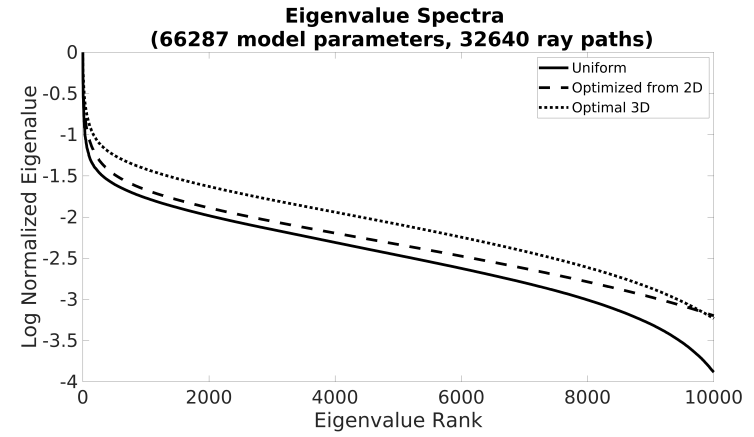

Figure 6.7.: Comparison of normalized eigenvalue spectra between the uniform design (solid line), the optimized design constructed from the set of 2D GOED problems using sagittal and coronal slices (dashed line) and the optimal design resulting from full 3D GOED (dotted line).

\section{(a) Reference setup}
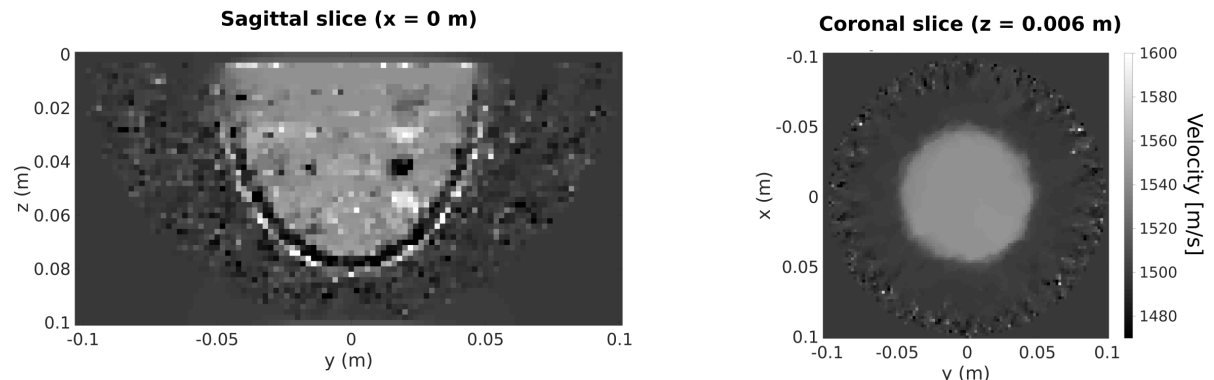

(b) Optimized setup from 2D OED
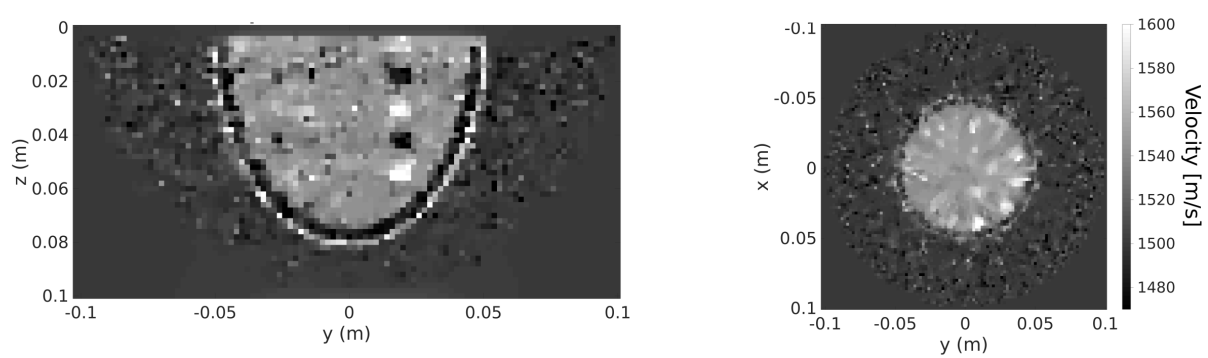

(c) Optimal setup 3D OED
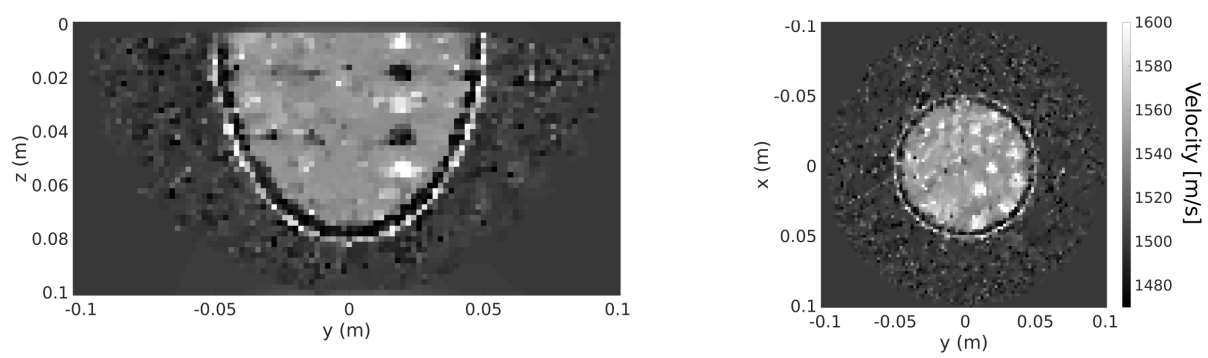

Figure 6.8.: (a)-(c) Sagittal $(x=0 \mathrm{~m})$ and coronal $(z=0.006 \mathrm{~m})$ slices showing the velocity reconstructions obtained from the experimental designs in Figure 6.6. Traveltime measurements include Gaussian noise with standard deviation of $0.2 \%$. 


\subsubsection{Validation of SOED approach 6}

To make the optimization computationally more tractable, we validate the performance of SOED algorithm by applying it to the same hemispherical system. In this way, we are able to compare the solutions found using both GOED and SOED method, shown in Figure 6.9. We observe that although GOED and SOED yield slightly different results, they both have similar patterns that include a ring of transducers close to the chest wall (i.e., near the equator of the hemisphere) and at least one arc of densely clustered transducers. The majority of the remaining transducers have an angular distance of at least $120^{\circ}$ from the main arc. Figure 6.10(a) shows the normalized eigenvalue spectra of the three different configurations, including the reference setup. The optimized designs obtained from GOED and SOED improve very similarly the spectrum of the reference setup. Not only they improve the relative magnitudes of the eigenvalues, but they also increase the number of significant eigenvalues. As pointed out in the previous section, a possible limitation of SOED is that it is a so-called greedy algorithm, which - by construction - always selects the next best location, but does not necessarily yield the globally best design for a given number of transducers. Figure 6.10(a) shows that GOED indeed improves the spectrum for small eigenvalues compared to SOED, but the differences are marginal. Furthermore, SOED has two important advantages over GOED. On the one hand, the computational cost of SOED is significantly smaller, and on the other hand, SOED enables us to analyze the benefit-cost curve to determine a suitable number of transducers, whereas this number has to be fixed a priori for GOED to make it computationally tractable. This curve is shown in Figure 6.10(b), where we represent the normalized quality measure with respect to the number of transducers in each iteration of SOED. Here, the dashed line indicates the number of transducers considered for this study. The curve shows that we do not gain a significant improvement in the quality measure by adding more transducer to the experiment, and therefore, they would only provide redundant data.

\footnotetext{
${ }^{6}$ This subsection has been modified from: H. Maurer, A. Nuber, N. Korta Martiartu, F. Reiser, C. Boehm, E. Manukyan, C. Schmelzbach and A Fichtner (2017). Optimized Experimental Design in the Context of Seismic Full Waveform Inversion and Seismic Waveform Imaging. Advances in Geophysics.
} 
(a) GOED

(b) SOED

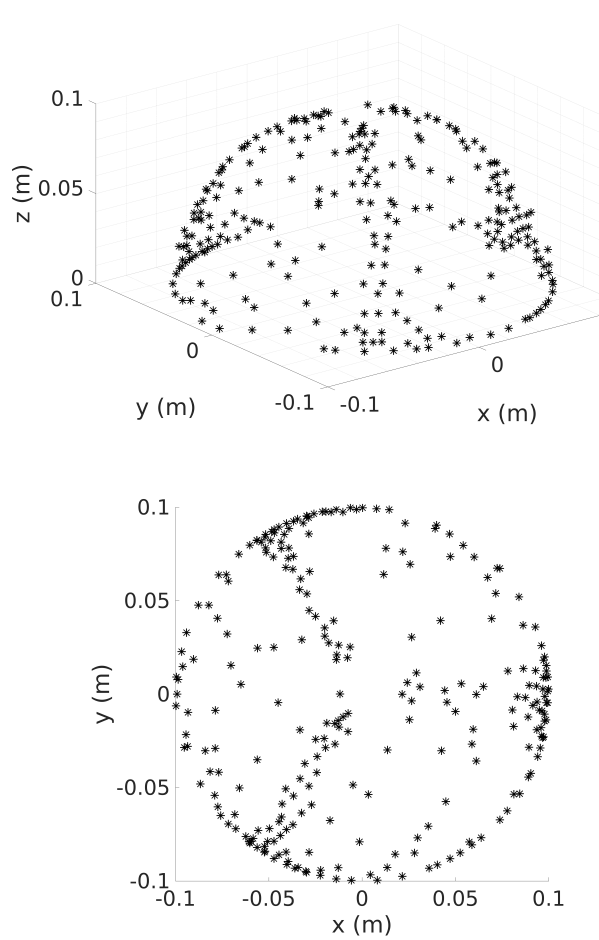

Lateral view

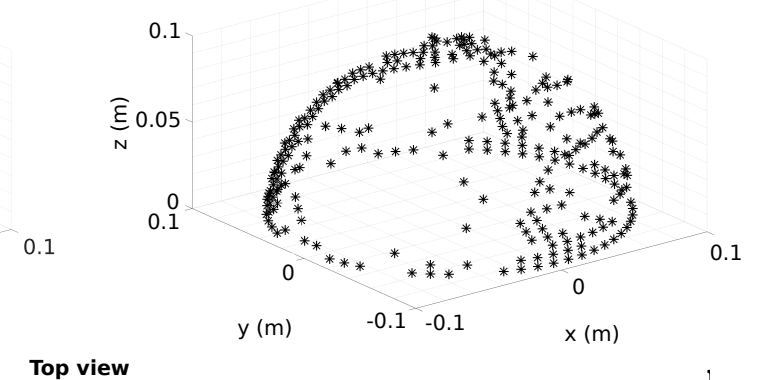

Top view

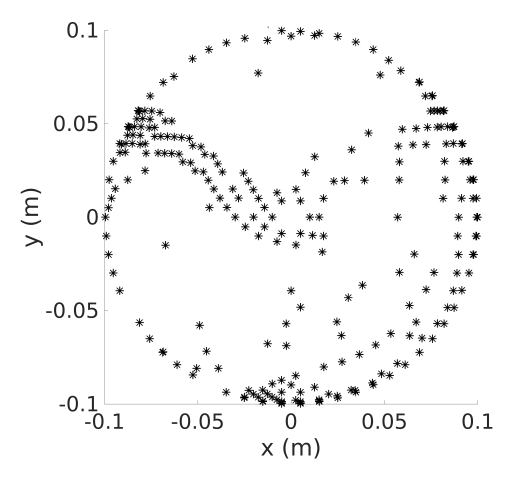

Figure 6.9.: Optimal experimental design obtained from (a) GOED and (b) SOED method.

(a)

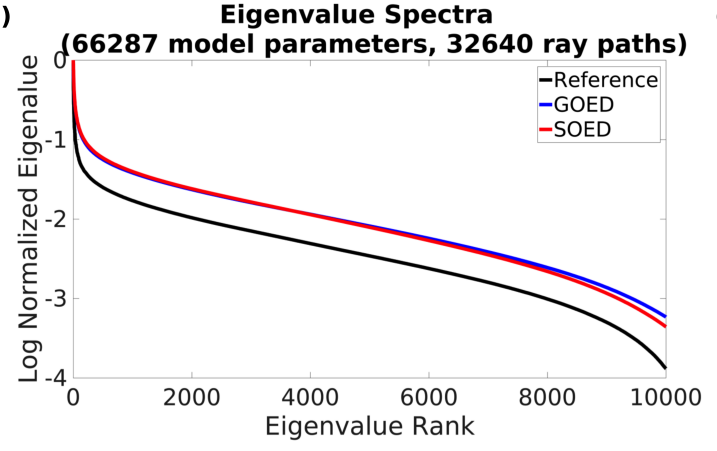

(b)

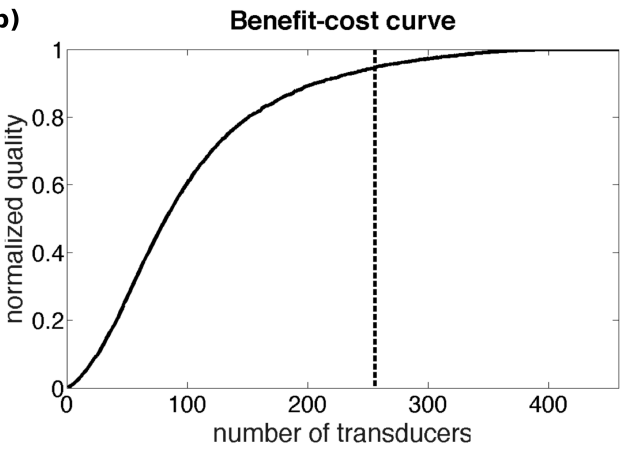

Figure 6.10.: (a) Normalized eigenvalue spectra for the reference setup and the solutions from GOED and SOED. (b) Benefit-cost curve obtained from SOED. Dashed line indicates the number of transducers used in our particular problem. 


\subsection{Discussion}

In this chapter, we introduce the OED method to the context of USCT and apply to determine the optimal transducer locations in a 3D hemispherical configuration. While other aperture shapes are also possible, this setup allows use to simplify the parameterization of transducer locations. The efficiency of the method has already been demonstrated extensively in geophysical applications. However, most of these problems are tackled by 2D inversion approaches, which avoid the huge computational cost related to large-scale 3D problems. To this end, we propose an approach that takes advantage of the radial symmetry of the scanning device.

The optimal design found using GA, shown in Figure 6.6(c), may not correspond to the global minimum of the design space. However, we run the global optimization several times, and the following features are common to all solutions:

(i) many transducers are placed in the region close to the chest wall while their density is decreased gradually with elevation;

(ii) a rotational symmetry of order three with respect to the z-axis is found where most transducers are placed in only three specific regions of the hemisphere.

Similar behavior has also been observed in 2D approximations of the experimental design that we perform in sagittal and coronal directions [see Figures 6.5(b) and 6.5(d), respectively]. In fact, our results in sagittal direction are comparable to the ones obtained by Curtis (1999a) for the crosswell tomography surveys in seismic applications. The authors show that the distribution of sources and receivers in each borehole is gradually increasing its density towards the base of the well (analogous to the chest wall in our case). These common features may therefore be used as rules of thumb in future experimental designs.

Whereas the first feature is due to the poor ray coverage of the uniform design in the region close to the chest, which is in fact of special interest in breast cancer diagnosis, the second feature is less intuitive. To understand this better, we consider only one transducer in emitting mode as shown in Figure 6.11(a), indicated by a square. To facilitate the interpretation, we consider a $2 \mathrm{D}$ approximation of the problem in the coronal plane at $z=0.006 \mathrm{~m}$, near the equator of the hemisphere. By definition, the quality measure in Equation (6.2) only considers ray paths intersecting with our ROI (indicated by a dashed circle). This is a consequence of removing from the forward operator $\mathbf{F}^{\prime}$ the columns corresponding to parameters outside the ROI. If we compute the tangent ray paths (solid lines) to our ROI, they will represent the limits of the region in the device in which transducers in receiving mode collect information about the breast. In other words, transducers placed outside of these limits would give us ray paths that do not cross our ROI and, therefore, contain no information about the breast. The angular distance between the limits measured 


\section{Designing USCT experiments: Part I}

from the center of the ROI (dashed lines) is $120^{\circ}$, which is consistent with the symmetry shown by the optimal experimental design [see Figure 6.11(b)].

Some of the patterns discussed above appear also in the result obtained from SOED algorithm. This not only confirms our previous observations in the optimized setup using GOED, but also validates the efficiency of SOED algorithm in producing solutions close to the ones obtained by global optimizations. We therefore suggest to use SOED method in future studies, since it provides a computationally more tractable alternative for optimizing experimental designs. It moreover has the potential to analyze redundancies in the measurements using benefit-cost curves, which is an advantage over the GOED method. In this study, we consider a fixed number of only 256 transducers to ensure a low cost experimental design, understanding that rotations allow us to have additional virtual locations for them. The benefit-cost curve obtained from SOED confirms that this number provides a suitable benefit-cost ratio for this experiment.
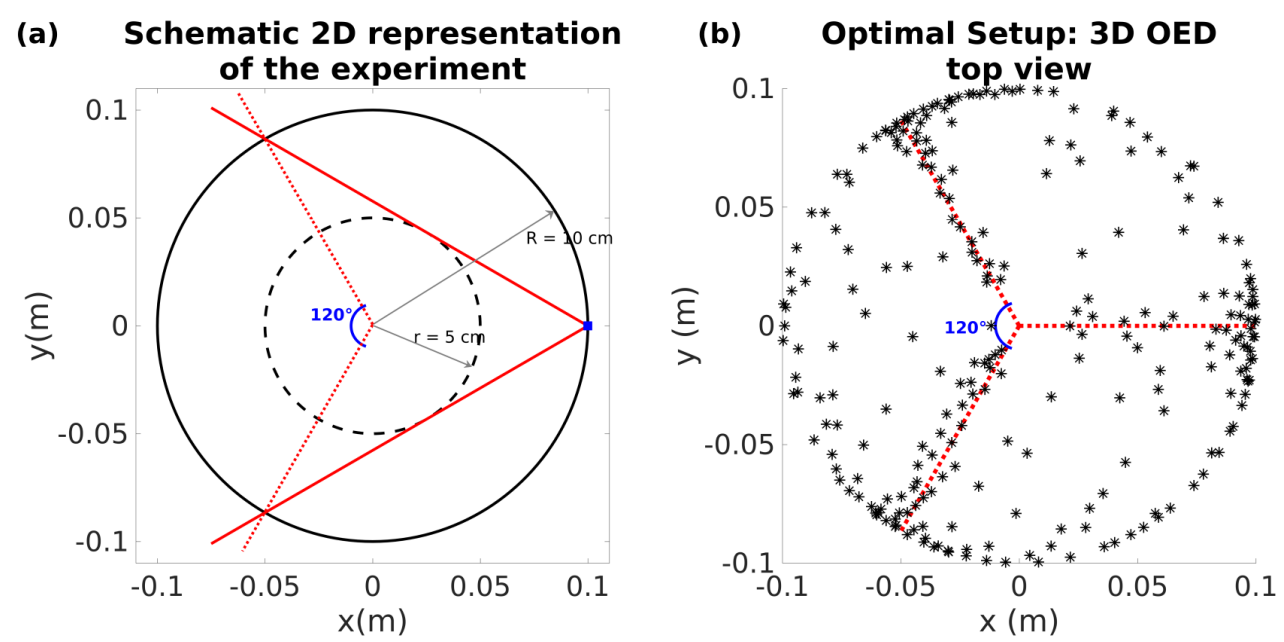

Figure 6.11.: (a) Given an emitting transducer (square), the maximum angle between two receiving transducers for which ray paths (solid lines) intersect with our ROI (dashed circle). The solid circle represents the device holding the transducers. (b) Top view of the optimal experimental design found in this study. Dashed lines with angular separation of $120^{\circ}$ are overlaid to the final result.

The techniques presented herein mainly consider traveltime inversions for reconstructing the velocity of breast tissue. Therefore, only transmitted data was taken into account to determine the optimal design of the experiment. Extensions of the OED problem to other linear(ized) problems introduced in previous chapters is straightforward and will be studied in the next Chapter 7. In Section 6.1, however, we also discussed extensions to non-linear problems, in particular to waveform tomography.

It is difficult to assess to which extend the traveltime configuration can also yield an 
improved configuration for waveform inversion. Clearly, there are many more parameters involved, e.g., frequency of the transducers, selection of emitting transducers, emitted signal, misfit functional, simulation time, etc., that influence the result. To analyze this, we estimate the trace of the data-misfit Hessian for the reference and optimized configurations in $2 \mathrm{D}$ sagittal direction. The results are shown in Table 6.2, and we observe that the optimal design for traveltime inversion indeed yields an improvement for waveform tomography compared to the uniform configuration. The estimated trace of the Hessian, computed with adjoint methods and low-frequency transducers, is 3.85 for the optimized and 3.46 for the uniform design. However, verifying the results in $3 \mathrm{D}$ is computationally challenging due to the tremendous cost of numerical simulations of wave propagation in heterogeneous media required to approximate the trace of the Hessian using many emitters. Therefore, we consider three different experiments with either only one or 40 emitter with locations that are randomly selected from either the uniform or the traveltime optimal configuration. The results are summarized in Table 6.2. In general, both configurations give similar results in all tests, with each design giving slightly better values in two of the experiments. However, because we only consider a small subset of emitters, interpreting these results is difficult. In the next Section 6.5, we discuss improved approaches to OED in waveform tomography and show some preliminary results.

\begin{tabular}{|c|c|c|}
\hline Sources & Reference Setup & Optimal Setup \\
\hline 2D, sagittal slice & 3.46 & 3.85 \\
3D, single source at $z=0.075 \mathrm{~m}$ & 4.86 & 4.83 \\
3D, single source at $z=0.097 \mathrm{~m}$ & 4.90 & 5.00 \\
3D, 40 sources & 15.17 & 15.03 \\
\hline
\end{tabular}

Table 6.2.: Estimates of the trace of the Hessian evaluated for waveform tomography using either the reference setup or the optimal setups from traveltime inversion as shown in Figures 6.5(b) and $6.6(\mathrm{c})$, respectively.

\subsection{Conclusions}

We applied advanced methods of optimal experimental design to improve the transducer configuration for USCT. This provides a systematic and quantitative framework to improve the spatial resolution and uncertainties of the reconstructions without the need for additional measurements. Furthermore, these methods allow us to gain intuition about the design and to significantly reduce the computational cost of the inverse problems.

We observe that exploiting the radial symmetry enables us to start with a sequence of $2 \mathrm{D}$ problems that is then extended to a fully $3 \mathrm{D}$ design. The reconstructed images show the great potential of using non-uniform transducer locations in 3D ultrasound tomography 


\section{Designing USCT experiments: Part I}

scanning devices. We discovered an interesting pattern that clusters the transducers on a ring close to the chest wall and in three distinct areas on the hemisphere. The applicability of this design for waveform inversion is subject of future research. Further extensions of the method are possible to different design parameters (e.g., rotations, number of transducers) or tissue properties trying to constrain reflectivity, attenuation or density.

\subsection{Outlook: practical extension to waveform tomography}

Waveform tomography is a non-linear inverse problem, and therefore, the data-misfit Hessian is model dependent. That is, the values of the quality measure depend on the model that is used to evaluate it. This clearly produces considerable challenges when optimizing the experimental setup previous to any acquisition since the model parameters are unknown at that stage. Therefore, we typically assume that the Hessian does not significantly change for models that are sufficiently close to the global minimum of the objective function $\chi(\mathbf{m})$. We have discussed this in Chapter 4, and in Section 4.3 in particular, and during this chapter we used the quadratic approximation of $\chi(\mathbf{m})$ in the vicinity of the global minimum to access the posterior covariance operator through the data-misfit Hessian. A computationally more tractable alternative, however, is to assume the Gauss-Newton approximation of the Hessian, i.e., to linearize the forward operator of waveform tomography in the vicinity of the global solution [see Equations (4.63) and (4.64)]. Crestel et al. (2017) compared this approximation to the full Hessian given by Equation (4.62) to study the application of OED to encoding sources in FWI. Their results suggest that the Gauss-Newton approximation preserves important information about the trace of the posterior covariance, while being moreover positive definite by construction. This allows us to use evaluation points $\mathbf{m}$ that are further from the global minimum of the objective functional $\chi(\mathbf{m})$.

Inspired by these results, we suggest an extension of OED methods to waveform tomography using the following simplifying assumptions and approximations. First, we linearize the forward problem and work only with the Gauss-Newton approximation of the Hessian evaluated at the initial model parameters $\mathbf{m}$. This is equivalent to using the Born approximation, which considers only single-scattering effects. Second, we approximate the information content based on the properties of the trace of the posterior covariance, i.e.,

$$
\Theta=\operatorname{trace}\left(\boldsymbol{\Gamma}_{\text {post }}\right)=\operatorname{trace}\left(\mathbf{H}^{-1}\right),
$$

which is known as the A-optimality condition (Haber et al., 2008; Alexanderian et al., 2016) and can be understood as minimizing the average variance of the parameters to estimate. Finally, we do not solve the experimental design problem exactly but with the help of SOED. In the following, we show some preliminary results using this approach. 


\subsubsection{Numerical example}

We use a 2D numerical example to analyze the performance of the suggested approach and compare the A-optimality condition to the trace of the approximate Hessian that was discussed in Section 6.1.1. We consider the breast model and experimental setup shown in Figure 6.12. The breast model corresponds to a coronal slice of the 3D Derenzo phantom in the upper part close to the chest wall, where the region of interest is restricted to a central disk of radius $6 \mathrm{~cm}$ and parameterized using a rectilinear grid of $2 \mathrm{~cm}$ mesh size. The experimental setup consists of a $2 \mathrm{D}$ ring-shaped device of radius $10 \mathrm{~cm}$. The circles indicate the possible locations of the transducers, 62 in total, which can act as both emitters or receivers and from which we aim to select the 16 optimal ones. When acting as emitter, we consider them as point sources, modelled using a Ricker wavelet with a center frequency of $200 \mathrm{kHz}$. The initial model is a homogeneous medium representing water, and we use the software Macesopt for the numerical wave propagation simulations and image reconstructions (Boehm, 2015).

Figures 6.12(b) and 6.12(c) show the optimal transducer configuration obtained using as quality measure the trace of the Gauss-Newton Hessian and A-optimality condition, respectively. As we observe, the trace of the approximate Hessian as a quality criterion gives a poor configuration focusing only on specific parts of the region of interest. On the contrary, the A-optimal design places the transducers quite evenly along the ring providing a good coverage in the whole region of interest. Figure 6.12(b) contradicts our observation made in Table 6.1. This may be a consequence of the approximation introduced by the Gauss-Newton Hessian, which neglects the multiple-scattering effects included in the second term of the full Hessian [see Equation 4.62]. The reconstruction obtained using A-optimally selected 16 transducers contains most of the anomalies of the true phantom, and demonstrates the efficiency of the approach presented here. It moreover suggests that including more transducers will mostly add redundant information. Because the setup is optimized considering water as the background model, this result also confirms that, for this particular application, the Gauss-Newton Hessian provides sufficiently good approximations of the relevant structures of $\chi(\mathbf{m})$ in the neighborhood of its global minimum.

Optimized configurations for both quality measures are also studied in a $2 \mathrm{D}$ example in sagittal direction, shown in Figure 6.13. In this case, we select the 18 most informative transducers out of 61 possible locations. The results confirm our previous observations made in coronal direction: A-optimality condition gives superior results validated with a reconstruction that recovers more accurately the properties of the true phantom. Furthermore, Figure 6.14 displays the eigenvalue spectra corresponding to the solutions obtained using both quality measures, and for both coronal and sagittal directions. A-optimal designs provide spectra with more balanced information than the designs obtained from maximizing the trace of the approximate Hessian. Consequently, the number of signif- 
(a) Setup \& Model
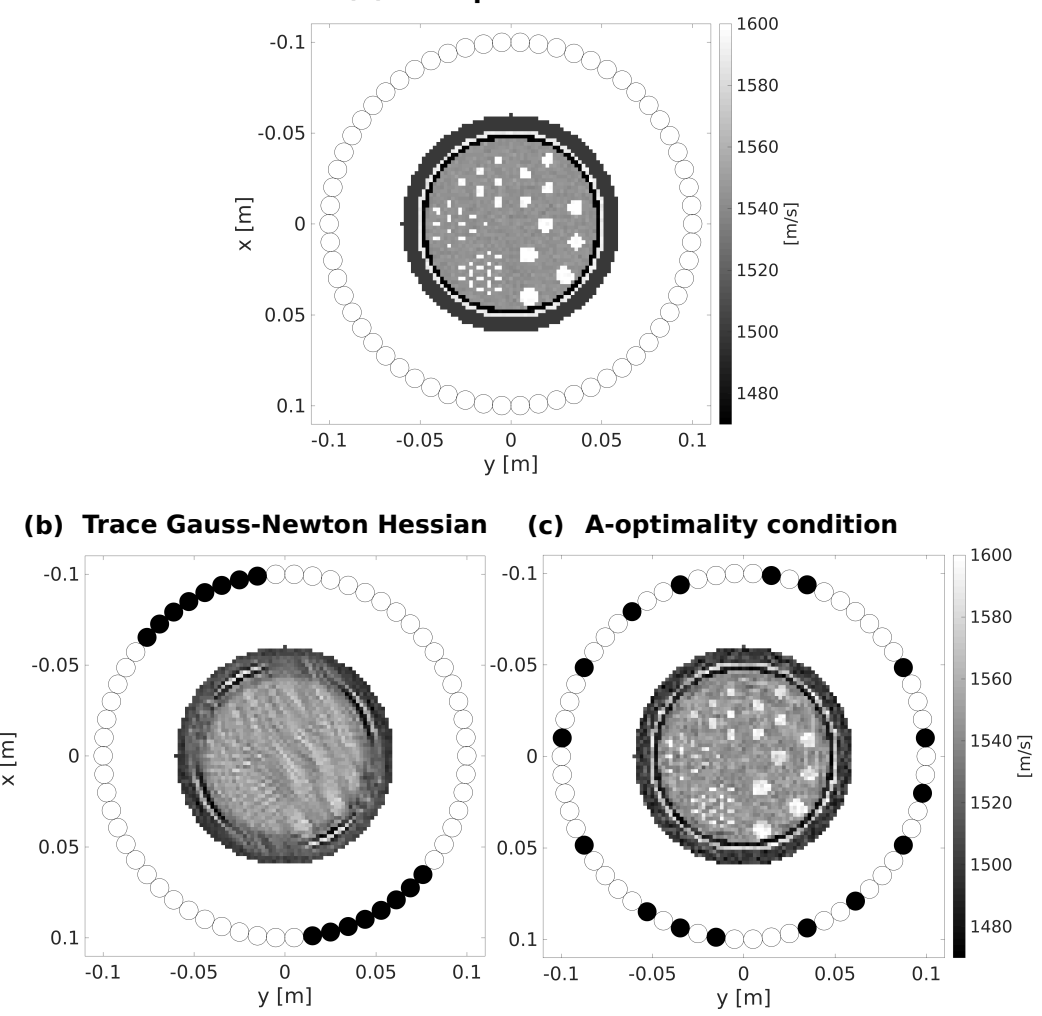

Figure 6.12.: (a) Acquisition setup and numerical phantom considered for this example. The circles indicate the possible locations of the transducers (62), and the white region illustrates the parts of the domain that are outside the ROI. (b), (c) Optimally selected transducers (black circles) using the trace of the approximate Hessian and the A-optimality condition, respectively. The ROI shows the reconstruction obtained by the optimized transducer configuration in each case.

icant eigenvalues is increased for A-designs. Here, we also show the eigenvalue spectra for uniform designs (regularly spaced transducers) in both directions. Interestingly, the eigenvalues are very similar for uniform and A-designs. This suggests that the quality of the reconstructions obtained from FWI is not very sensitive to transducer locations, at least for experiments with sufficiently even configurations. In such context, OED methods are more useful (1) to identify redundancies in the measurements and, therefore, reduce the inherent computational cost of FWI by selecting the most informative transducers, and (2) to ensure a good starting models by optimizing the experiment for linear approaches. Identifying redundancies in linear problems will be the topic of the next chapter, in which we further extend the OED method for joint reflection-transmission imaging. We refer the reader to Maurer et al. (2017) for a review of studies applying OED in the context of FWI, with particular focus on seismic applications. 
(a) Setup \& Model

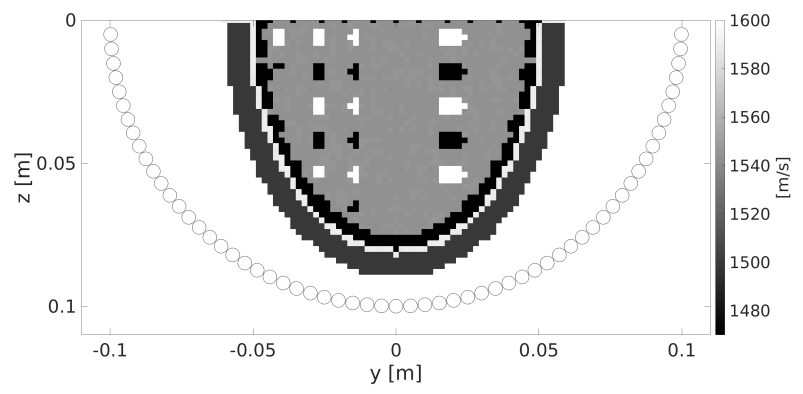

(b) Trace Gauss-Newton Hessian

(c) A-optimality condition

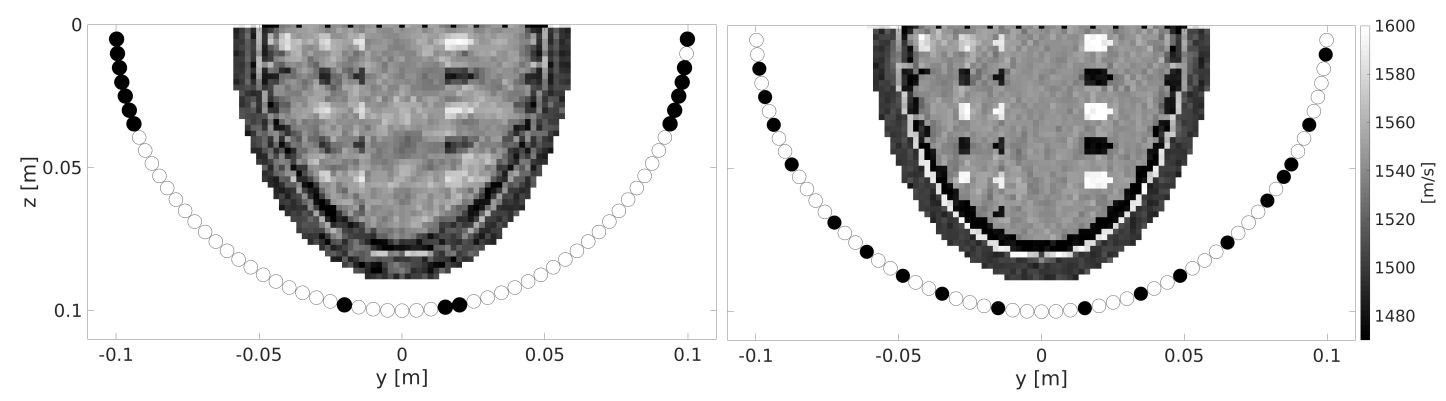

Figure 6.13.: (a) Acquisition setup and numerical phantom considered for the example in sagittal direction. There are a total of 61 possible locations for the transducers. (b), (c) Optimally selected transducers (black circles) using the trace of the approximate Hessian and the A-optimality condition, respectively. The ROI shows the reconstruction obtained by the optimized transducer configuration in each case.

\section{Coronal slice}

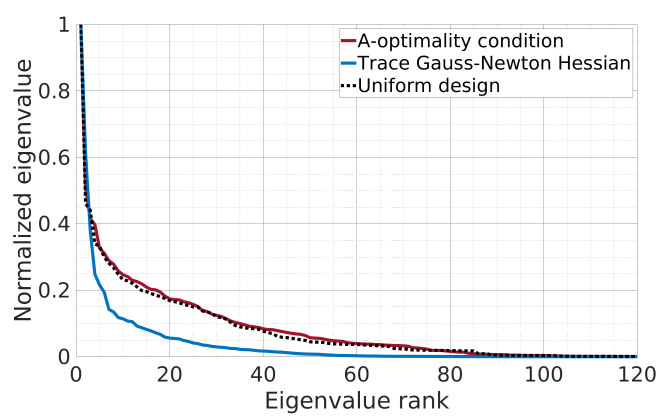

Sagittal slice

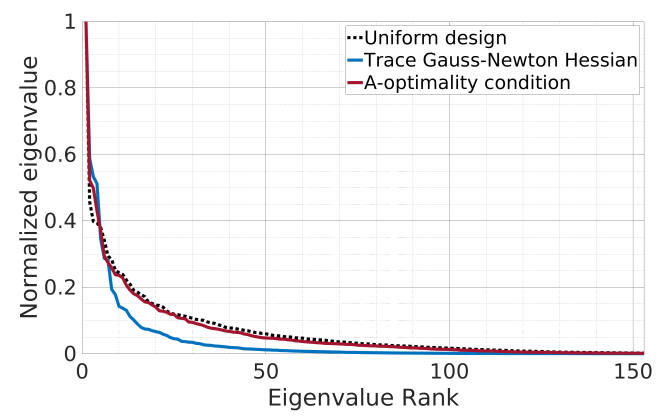

Figure 6.14.: Comparison between eigenvalue spectra of optimized and uniform designs in (left) coronal and (right) sagittal directions. To better visualize the differences, the eigenvalues are normalized by the largest eigenvalue in each case and the eigenvalue rank is truncated showing the ones with meaningful values. 



\section{Designing USCT experiments: Part II§}

As noticed in the previous chapter, the optimal design of an experiment depends on the specific image reconstruction method intended to be applied post-acquisition. This makes it difficult to guarantee that acquisition systems designed for specific imaging methods will benefit other methods as well. We have observed this, for instance, when comparing ray theoretical approaches with waveform tomography. In USCT systems, however, multimodality is essential. It allows us to identify lesions using information from multiple properties of the breast tissue, and these are obtained, in general, by applying different imaging techniques. A suitable USCT device must therefore find the best compromise for several image reconstruction methods, providing moreover high accuracy and affordable computational and acquisition cost for clinical practice.

Sequential optimal experimental design (SOED) offers a systematic framework to account for the interdependency between (1) the quality of the reconstructed images, (2) the involved computational cost, (3) the applied tomographic method, and (4) the design of the experimental setup. This is key to find an optimal trade-off between accuracy and cost, and sufficiently flexible and powerful to integrate multi-modality aspects. The latter include techniques that exploit either transmission or reflection information from the observed signals, and therefore, an optimal scanning system should simultaneously provide accurate results for both. Using SOED method, we optimize the transducer configuration by minimizing the uncertainties in the estimated model parameters, and we reduce the time to solution by identifying redundancies in the data. Uncertainties in the parameters can be quantified by extracting properties of the posterior covariance operator, which is analytically computed by linearizing the forward problem with respect to the prior knowledge about parameters.

This chapter presents a formulation to jointly optimize the experiment for transmission and reflection data, and in particular, to estimate the velocity and reflectivity of the tissue using either ray-based or wave-based imaging methods. This can be done by computing the joint posterior covariance operator that considers multiple imaging methods. In the following, we first introduce the joint formulation and a suitable quality measure that

\footnotetext{
$\S$ The content of this chapter has recently been published as: N. Korta Martiartu, C. Boehm, V. Hapla, H. Maurer, I. Jovanović Balic and A. Fichtner (2019). Optimal experimental design for joint reflectiontransmission ultrasound breast imaging: from ray- to wave-based methods. The Journal of the Acoustical Society of America.
} 
balances the contribution of different techniques. Then, we illustrate the performance of our SOED approach using a numerical toy example, and we conclude by applying it to real data. This shows that the time to solution can be substantially reduced, without altering the final image, by selecting the most informative measurements.

\subsection{Joint optimization of the experimental design}

\subsubsection{Joint posterior covariance}

Ideally, an experimental setup should be flexible enough to provide reliable reconstructions of different acoustic parameters using multiple tomographic methods. Assume, for instance, that we expect to reconstruct the velocity $\mathbf{m}_{1}$ and the reflectivity $\mathbf{m}_{2}$ of the tissue using the imaging techniques $\mathbf{F}_{1}$ and $\mathbf{F}_{2}$, respectively. Moreover, we denote by $\mathbf{d}_{1}$ and $\mathbf{d}_{2}$ the corresponding observed data, and by $\boldsymbol{\Gamma}_{1}$ and $\boldsymbol{\Gamma}_{2}$ the covariance matrix of the noise for each case, respectively. As usual, we assume both to be Gaussian with zero mean. We can formulate the joint forward problem as

$$
\left[\begin{array}{l}
\Delta \mathbf{d}_{1} \\
\Delta \mathbf{d}_{2}
\end{array}\right]=\left[\begin{array}{cc}
\mathbf{F}_{1}^{\prime} & \mathbf{0} \\
\mathbf{0} & \mathbf{F}_{2}^{\prime}
\end{array}\right]\left[\begin{array}{l}
\Delta \mathbf{m}_{1} \\
\Delta \mathbf{m}_{2}
\end{array}\right]
$$

which essentially consists of two separable subproblems. Here we have simplified the notation as $\mathbf{F}_{i}^{\prime}=\mathbf{F}_{i}^{\prime}(\mathbf{s})$ for clarity. If we now define $\boldsymbol{\Delta} \mathbf{d}_{\text {obs }}=\left[\boldsymbol{\Delta} \mathbf{d}_{1} ; \boldsymbol{\Delta} \mathbf{d}_{2}\right]$ and $\boldsymbol{\Delta} \mathbf{m}=$ $\left[\boldsymbol{\Delta} \mathbf{m}_{1} ; \boldsymbol{\Delta} \mathbf{m}_{2}\right]$, and we denote the joint forward operator as $\mathbf{F}^{\prime}$, the posterior covariance matrix will be given by Equation (4.34), i.e.,

$$
\boldsymbol{\Gamma}_{\text {post }}(\mathbf{s})=\left(\mathbf{F}^{\prime}(\mathbf{s})^{T} \boldsymbol{\Gamma}_{\text {noise }}^{-1} \mathbf{F}^{\prime}(\mathbf{s})+\boldsymbol{\Gamma}_{\text {prior }}^{-1}\right)^{-1} .
$$

In this case, the data-misfit Hessian is a block-diagonal matrix

$$
\mathbf{H}_{\text {misfit }}(\mathbf{s})=\mathbf{F}^{\prime T} \boldsymbol{\Gamma}_{\text {noise }}^{-1} \mathbf{F}^{\prime}=\left[\begin{array}{cc}
\mathbf{F}_{1}^{\prime T} \boldsymbol{\Gamma}_{1}^{-1} \mathbf{F}_{1}^{\prime} & \mathbf{0} \\
\mathbf{0} & \mathbf{F}_{2}^{\prime T} \boldsymbol{\Gamma}_{2}^{-1} \mathbf{F}_{2}^{\prime}
\end{array}\right]=\left[\begin{array}{cc}
\mathbf{H}_{\text {misfit }, 1} & \mathbf{0} \\
\mathbf{0} & \mathbf{H}_{\text {misfit }, 2}
\end{array}\right] \text {, }
$$

with $\boldsymbol{\Gamma}_{\text {noise }}^{-1}$ being a block-diagonal matrix composed by $\boldsymbol{\Gamma}_{1}^{-1}$ and $\boldsymbol{\Gamma}_{2}^{-1}$.

As a first approximation, our formulation neglects the trade-offs between parameter $\mathbf{m}_{1}$ and $\mathbf{m}_{2}$, which is justified by the specific nature of each subproblem. This point has already been discussed in Section 4.1.3.

\subsubsection{Quality measure}

In the previous Chapter, we have applied quality measures that involve the trace of either $\boldsymbol{\Gamma}_{\text {post }}$ or $\mathbf{H}_{\text {misfit }}$. This quantity is easy to access and it only requires the diagonal 
entries of the matrix. However, largest eigenvalues may dominate the trace, and this is undesirable for joint optimizations, where we need to balance the contribution of different imaging techniques. In this study, we consider the D-optimality condition (Alexanderian and Saibaba, 2018; Atkinson and Donev, 1992; Attia et al., 2018), which defines $\Theta$ as the determinant of the posterior covariance operator $\boldsymbol{\Gamma}_{\text {post }}$. Because the determinant may show very small values, D-optimal design is often found by minimizing the logarithm of the determinant,

$$
\Theta=\log \operatorname{det}\left(\boldsymbol{\Gamma}_{\text {post }}\right)=-\log \operatorname{det}\left(\mathbf{H}_{\text {misfit }}+\boldsymbol{\Gamma}_{\text {prior }}^{-1}\right) .
$$

One can compute this quantity using Cholesky factorization of $\mathbf{H}_{\text {misfit }}+\boldsymbol{\Gamma}_{\text {prior }}^{-1}$, which is positive definite by construction. Note that $\boldsymbol{\Gamma}_{\text {prior }}^{-1}$ acts as a threshold for small eigenvalues of $\mathbf{H}_{\text {misfit }}$. We can alleviate the cost of computing $\Theta$ by reducing the domain to a subset of parameters. This is particularly helpful to maximize the information about a specific ROI.

The determinant corresponds to the product of the eigenvalues, and thus, the D-optimal design minimizes the volume of the uncertainty ellipsoid in the estimated parameters. From information theory, this quantity can be related to the expected information gain $\Psi$ (Attia et al., 2018), which is an important property for our application. We compute this by subtracting from $\Theta$ the contribution of the prior $\boldsymbol{\Gamma}_{\text {prior }}$, i.e., $\Psi=-\Theta+\log \operatorname{det}\left(\boldsymbol{\Gamma}_{\text {prior }}\right)$. Furthermore, the D-optimal designs show invariant properties with respect to different model reparameterizations (Dette and O'Brien, 1999). This may be crucial when the parameterization for the reconstructions is not known beforehand. Here, we refer to the discretization of the acoustic tissue parameters as parameterization.

For joint optimizations introduced in the previous section, the quality measure is the combination of terms related to each subproblem,

$$
\Theta=-\log \operatorname{det}\left(\mathbf{H}_{\text {misfit }, 1}+\boldsymbol{\Gamma}_{\text {prior }, 1}^{-1}\right)-\log \operatorname{det}\left(\mathbf{H}_{\text {misfit }, 2}+\boldsymbol{\Gamma}_{\text {prior }, 2}^{-1}\right) .
$$

Note that the data and prior covariances weight the contribution of both terms. We further discuss this aspect in Section 7.2.

\subsubsection{Non-Gaussian priors}

While non-Gaussian priors are often used in USCT ( $L i$ et al., 2009; Matthews et al., 2017), in this study we rely on Gaussian priors to formulate the D-optimal SOED (DSOED) approach. In this way, analytical expressions of the posterior are available (see Equation (4.34)). To compute the D-optimality criterion, (1) we constrain the model parameters to the ROI, (2) we assume equally reliable observations, $\boldsymbol{\Gamma}_{\text {noise }}=\sigma_{n} \mathbf{I}$, and (3) we define $\boldsymbol{\Gamma}_{\text {prior }}=\sigma_{p} \mathbf{I}$, with $\sigma_{p}^{-1}$ small enough to stabilize the inversion of the illconditioned matrix $\mathbf{H}_{\text {misfit }}$. As a result, our algorithm selects the experimental designs improving most the properties of $\mathbf{H}_{\text {misfit. }}$. In particular, we expect the optimal designs to 


\section{Designing USCT experiments: Part II}

improve the effective rank of $\mathbf{H}_{\text {misfit }}$, which has a rapidly decaying spectrum. Because the regularization techniques mainly act in the effective nullspace of $\mathbf{H}_{\text {misfit }}$, reconstruction algorithms that use other priors will also benefit from our solutions.

\subsubsection{Application to imaging techniques}

The D-SOED strategy outlined above may be applied to any linearizable inverse problem. Here we specifically focus on four imaging methods introduced in Section 4.1, excluding amplitude tomography for attenuation. The model parameters to estimate are the velocity $c(\mathbf{x})$ and the reflectivity $I(\mathbf{x})=\frac{2 \delta c(\mathbf{x})}{c_{0}}, c_{0}$ and $\delta c(\mathbf{x})$ being the background velocity and the perturbations with respect to it, respectively. These parameters are retrieved from the observations of the pressure field $p\left(\mathbf{x}_{r}, t ; \mathbf{x}_{s}\right)$ at receiver positions $\mathbf{x}_{r}$, and in particular, we extract the information of the first-arrival times $T\left(\mathbf{x}_{s}, \mathbf{x}_{r}\right)$ and the scattered wavefields $\delta p\left(\mathbf{x}_{r}, t ; \mathbf{x}_{s}\right)$. Linking to the notation used to formulate the joint forward problem in Equation (7.1), we denote the unknown parameters as $\boldsymbol{\Delta} \mathbf{m}=\left[\boldsymbol{\Delta} \mathbf{m}_{1} ; \boldsymbol{\Delta} \mathbf{m}_{2}\right]=[\delta c(\mathbf{x}) ; I(\mathbf{x})]$, and the observed data as $\boldsymbol{\Delta} \mathbf{d}_{\mathrm{obs}}=\left[\boldsymbol{\Delta} \mathbf{d}_{1} ; \boldsymbol{\Delta} \mathbf{d}_{2}\right]=\left[\delta T\left(\mathbf{x}_{s}, \mathbf{x}_{r}\right) ; \delta p\left(\mathbf{x}_{r}, t ; \mathbf{x}_{s}\right)\right]$. The explicit Jacobian operators are given in Section 4.1.

In this study in particular, our goal is to optimize the experiment prior to any acquisition, using only the information available at this stage. For reverse-time migration technique, we observe that if we linearize the forward problem using the prior information $c_{0}(\mathbf{x})=c_{0}$, i.e., a homogeneous background model, we obtain the same forward operator as in Bmode imaging [see Equation (4.19)]. We therefore expect that, an optimized experimental configuration for B-mode, will also be optimized for RTM. In such situations, we rely on Equation (4.19) to maximize the information about the ROI that can be retrieved from reflection data, independent of the employed imaging technique.

In the following sections we illustrate the potential of the joint D-SOED approach, first using an intuitive toy example, and then with an application to real data provided by CSIC/USM as part of the USCT Data Challenge 2017 (Ruiter et al., 2017).

\subsection{Toy example}

In our toy example, we consider a circular configuration of radius $10 \mathrm{~cm}$ with 125 regularly spaced transducers, shown in Figure 7.1. Here, we only take one transducer as emitter (red dot), and the rest as receivers (white dots). The source signal is a broad-band pulse, with frequencies in the range $350 \mathrm{kHz}-1.2 \mathrm{MHz}$, and the recording time length is $0.14 \mathrm{~ms}$. The unknown parameters are discretized on a rectilinear grid of $1 \mathrm{~mm}$. Because our D-SOED algorithm does not depend on the model parameters, we only specify a ROI in which we want to minimize the uncertainties. In the interest of producing intuitively interpretable results, we define a relatively small ROI, indicated by the white circle of radius $1.5 \mathrm{~cm}$. 
Our goal is to apply D-SOED to identify the most informative receivers when recording both transmission and reflection data. First, we analyze the sensitivity of our acquisition system to the ROI. This will allow us to gain deeper insight into the relationship between the experimental design and the individual imaging methods. Second, we optimize the receiver configuration separately for each imaging technique. We use this to introduce the DSOED algorithm and to discuss the benefit-cost curves described in Section 6.1.2.2. Finally, we perform the joint transmission-reflection optimization of the experimental design.

\subsubsection{Sensitivity of the acquisition system}

To understand the information that our acquisition system can exploit from each imaging technique, we first illustrate in Figure 7.1 the stacked sensitivities to model parameters of all emitter-receiver combinations, which can be interpreted as sensitivity coverage.

Figure 7.1(a) shows the sensitivity coverage for the traveltime tomography with straight rays. Only few receivers facing the emitter collect signals that contain information about the ROI, which in fact is very sparse. We therefore expect that the D-SOED algorithm will select these receivers as the most informative ones, giving a steep benefit-cost curve.

For finite-frequency tomography the information about the ROI is more uniform, as shown in Figure 7.1(b). Compared to Figure 7.1(a), receivers located at larger angles are also sensitive to the ROI. We therefore expect that the D-SOED algorithm will identify a larger number of informative receivers.

Figure 7.1(c) displays the sensitivity coverage for the reflection imaging technique. To understand this better, we moreover show in Figures 7.1(d) and 7.1(e) two examples of single measurements for receivers located on the reflection- and transmission-side, respectively. Both types of measurements are sensitive to the ROI, and the combination of both will cover the entire ROI. We therefore expect the D-SOED algorithm to select receivers at both sides, with even more density on the transmission-side due to the sparse information collected from there. Though this may initially appear counterintuitive, it is an effect of the recording time length, the location and size of the ROI, and the circular configuration of transducers.

\subsubsection{Individual optimizations}

We apply the D-SOED algorithm separately for each imaging technique. The resulting benefit-cost curves are shown in Figure 7.2(a). In all cases the information gain increases monotonically with the number of receivers, which means that more measurements improve the estimation of the parameters. At a certain point, however, the curves become flat. This occurs when the experiment includes receivers that either do not collect any information about the ROI or introduce redundancies. The transition between both stages, and in 


\section{Designing USCT experiments: Part II}

a) Straight-ray transmission b) Finite-frequency transmission
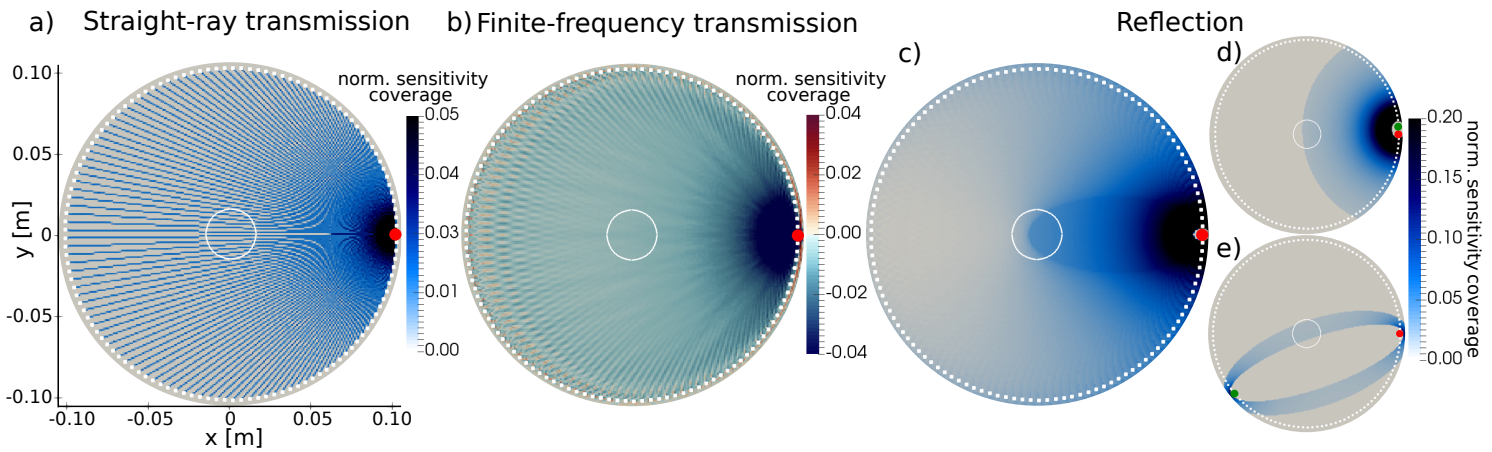

Figure 7.1.: Normalized sensitivity coverages for a circular configuration with 125 transducers. The white circle indicates the ROI, and the red and white dots are the emitter and receivers, respectively. (a) Traveltime tomography using straight rays; (b) Traveltime waveform tomography using a broad-band pulse of $350 \mathrm{kHz}-1.2 \mathrm{MHz}$; (c) Reflection imaging technique. (d), (e) Two examples of single measurement sensitivities for reflection data. Here, the green dots indicate the active receivers.

particular the point with the maximum curvature, represents the optimal benefit-cost ratio for the experiment, indicated by dashed lines in Figure 7.2(a).

Receiver configurations with the optimal benefit-cost ratio are displayed in Figures 7.2(b)-7.2(d). All results show the expected features discussed in Section 7.2.1. Because our ultimate goal is the joint optimization, we focus on comparing the optimized setups for transmission and reflection data. On the one hand, we do not observe any receiver that was simultaneously selected as the most informative one for transmission with straight rays and reflection. Indeed, the uninformative receivers for reflection data (identified in the flat part of the benefit-cost curve) are the most informative ones for tomography with straight rays. We therefore expect that the joint optimization between these two techniques will result in the superposition of both individual optimizations. On the other hand, when we consider finite-frequency tomography, there is an overlap between the most informative receivers for reflection and transmission. Here we expect that the D-SOED algorithm will select first the receivers in common for both imaging techniques. Due to this overlap, in the following we use the latter combination of techniques to discuss the joint D-SOED algorithm.

\subsubsection{Joint optimization}

We apply the D-SOED algorithm using the joint forward formulation in Equation (7.1) for finite-frequency tomography and reflection imaging. Figure 7.3(a) displays joint and individual benefit-cost curves for transmission and reflection, revealing three stages of the algorithm. Sensitivity coverages for each stage are shown in Figures 7.3(b)-7.3(g). First, the algorithm selects receivers that are simultaneously sensitive to the ROI for both 

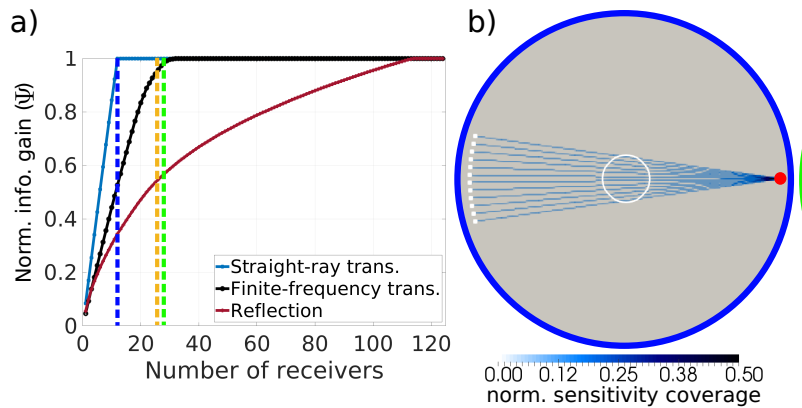

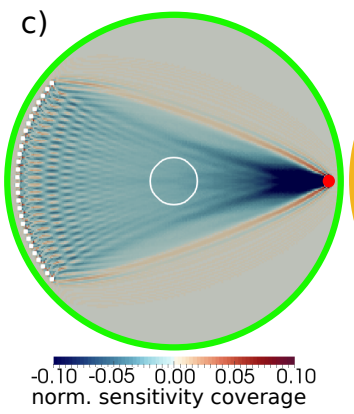

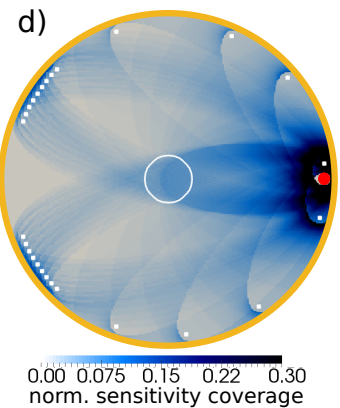

Figure 7.2.: Results from D-SOED method applied to each imaging technique. (a) Benefit-cost curves representing the information gain $\Psi$ with respect to the number of receivers. The dashed lines indicate the respective optimal number of receivers. (b)-(d) Normalized sensitivity coverages for the experiments using the most informative receivers obtained in each case: (b) traveltime tomography using straight rays (12 receivers), (c) traveltime waveform tomography (28 receivers), and (d) reflection imaging technique (26 receivers). The outlined circles link (b)-(c) to the dashed lines in (a).

transmission and reflection. Consequently, both individual benefit-cost curves increase with the number of receivers. Then, receivers informative only for the transmission technique are selected. The corresponding information gain increases while remaining almost constant for reflection data. Finally, the experimental design is completed with receivers on the reflection-side, which are uniquely informative through the reflection technique. Here, the transmission benefit-cost curve remains constant, and it increases monotonically for the reflection. During all these stages the joint information gain increases monotonically.

The data and prior covariances weight the contribution of each imaging technique in the joint optimization. Different choices will mostly affect the order in which the D-SOED algorithm selects the poorly informative receivers for each imaging technique. In our toy example, if the reflection technique contributes stronger to $\Theta$ in Equation (7.4), the receivers facing the emitter will be selected last. In other words, this would affect the second and third stages illustrated in Figure 7.3. However, we do not expect significant differences in the first stage, in which the algorithm selects first the receivers that contribute most for both techniques. Due to the nature of the determinant, the D-SOED algorithm essentially finds compromises between the parameters, providing solutions with balanced information.

\subsubsection{Comment on the parameterization}

One certainly could ask whether our results are influenced by the choice of the model parameterization, which in principle is undesirable when a particular one is not preferred. In Section 7.1.2, we have introduced the D-optimality condition as an attractive quality measure due to its invariance property to different choice for the discretization of the model. 

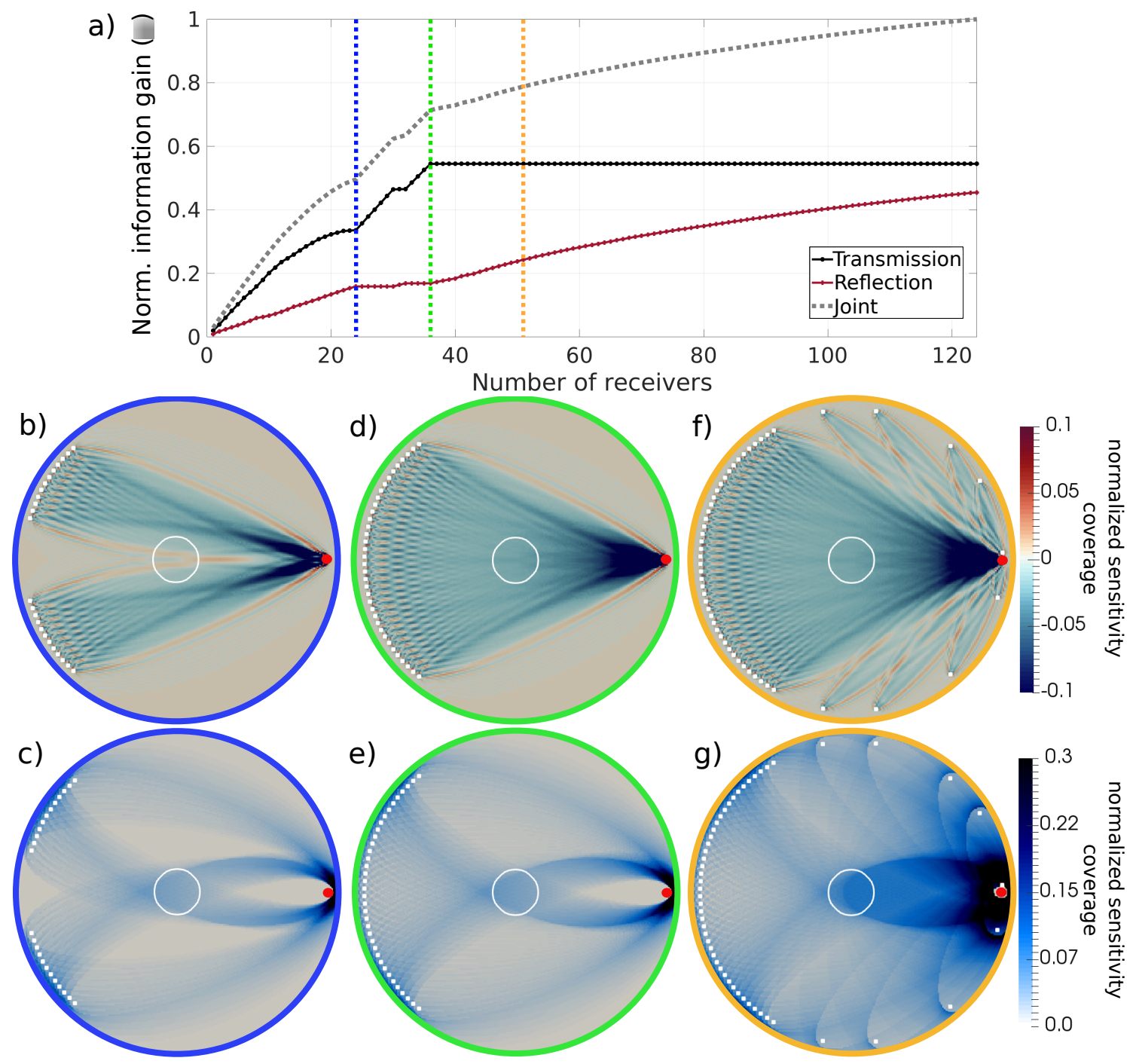

Figure 7.3.: Results from joint transmission-reflection D-SOED algorithm. (a) Joint and individual benefit-cost curves for each imaging technique. The curves are normalized by the total joint information gain $\Psi$. Blue, green and yellow dashed lines indicate 24, 36 and 51 (the optimal) number of receivers, respectively. (b), (d), (f) Transmission and (c), (e), (g) reflection sensitivity coverages for the optimized configurations with 24,36 and 51 receivers, respectively.

Although the values of the determinant may vary with the choice of the basis function used to discretize the space-continuous model, we do not expect changes in the characteristics of the benefit-cost curves.

Let us illustrate the invariance of the D-optimality with our toy problem. In the example above, the unknown parameters are discretized on a rectilinear grid of $1 \mathrm{~mm}$ mesh size. Now, in addition, we apply the D-SOED algorithm with parameters discretized on grids of 0.5 
$\mathrm{mm}$ and $2 \mathrm{~mm}$ mesh size. For this illustrative example, we perform individual optimizations similar to Section 7.2.2. The resulting benefit-cost curves are shown in Figure 7.4, where we display separately each parameterization as in Figure 7.2(a). As expected, for the three imaging techniques, the benefit-cost curves share common features for all parameterizations, including the optimal number of receivers identified from the maximum curvatures. This confirms that conclusions made from the D-SOED, which is previous to the acquisition, are independent to the discretization of the model that is applied post-acquisition. This has not only important implications for the reconstruction, but also can help to reduce the dimensionality, and thus, the complexity of experimental design problems.
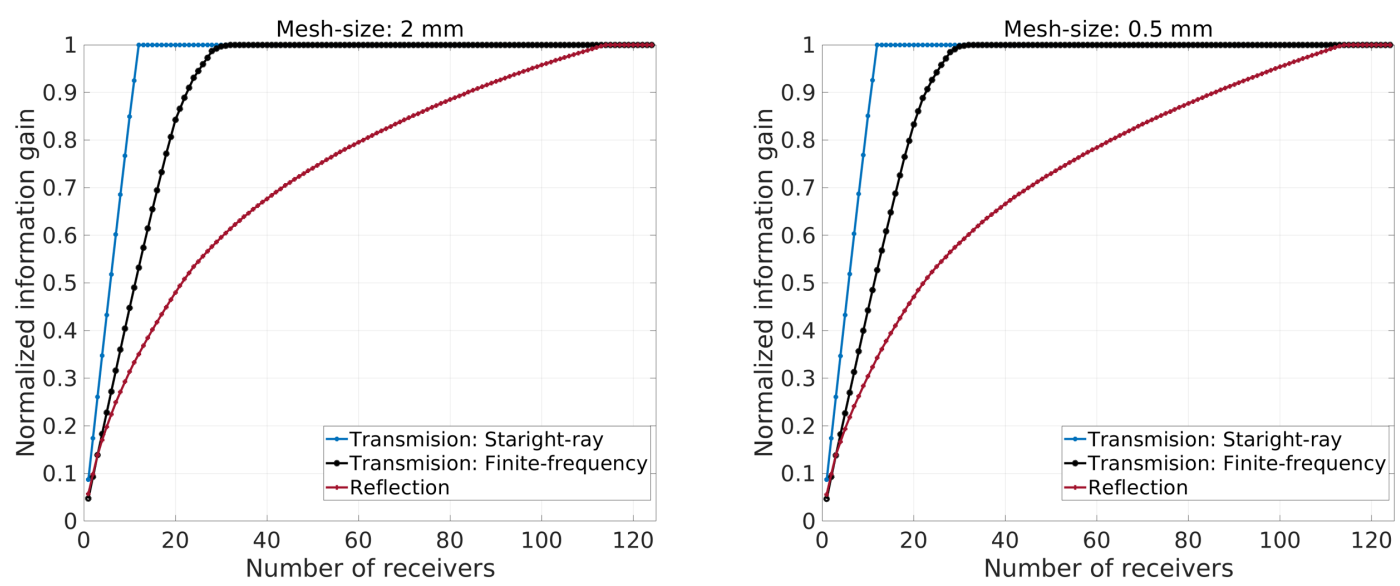

Figure 7.4.: Benefit-cost curves of individual optimizations of each imaging technique. The model is parameterized using a rectilinear grid of $2 \mathrm{~mm}$ (left) and $0.5 \mathrm{~mm}$ (right) mesh size.

\subsection{Real data application: emitter selection}

To validate our algorithm, we apply the D-SOED method to real data. We consider the dataset provided as part of the SPIE USCT Data Challenge 2017 by the Spanish National Research Council (CISC) and the Complutense University of Madrid (UCM). This was already described and applied in Section 5.2, see Figure 5.3. In this application, our goal is to decrease the data volume to reduce the time to solution. Here, the number of measurements is mainly controlled by the total number of emitting elements in the transducer array. Each element adds $23 \times 176=4048$ new measurements to the dataset. We therefore aim to select the most informative ones, removing those that provide redundant information about the ROI. This choice of the design parameter has an additional main reason: for imaging techniques based on numerical wave propagation (e.g., RTM or waveform tomography), the computational cost is proportional to the number of wave propagation simulations, 


\section{Designing USCT experiments: Part II}

and this is related to the number of emitters. Other design parameters are also possible, for instance the number of rotations or their angle.

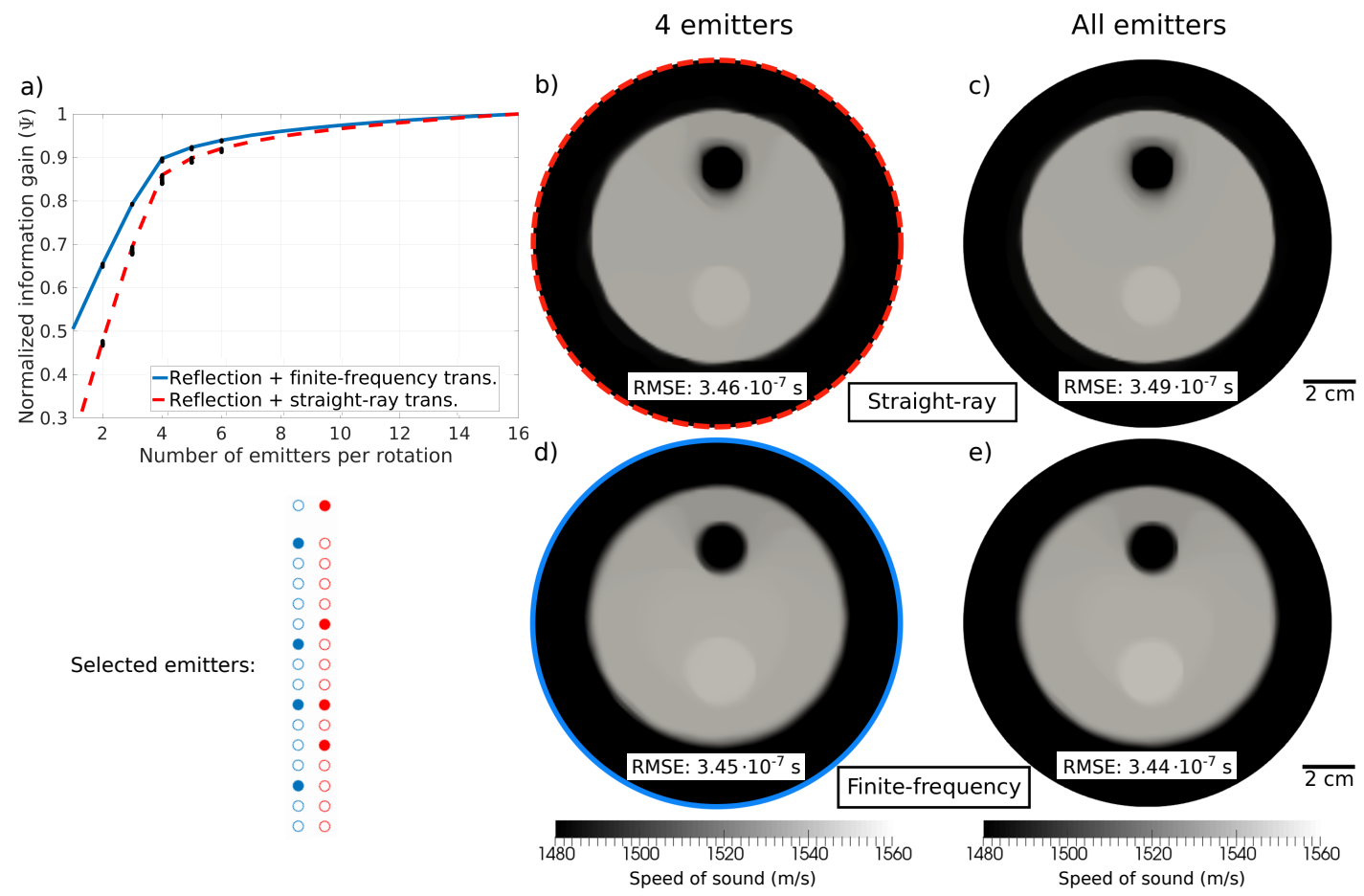

Figure 7.5.: (a) Benefit-cost curves of D-SOED for reflection-transmission when considering straight-rays (red) and finite-frequencies (blue). For 2 to 6 number of emitters, black lines indicate the variation in the information gain $\Psi$ for randomly selected emitter locations. (b), (c) Velocity reconstructions with straight-ray tomography using optimally selected 4 emitters and all the emitters, respectively. (d), (e) Velocity reconstructions using finite-frequency tomography. We use optimally selected 4 emitters and all the emitters, respectively. Selected emitters are illustrated in the bottomleft corner. The circles indicate the relative positions of the 16 elements in the emitter array (green balls in Figure 5.3), and the filled circles indicate the selected ones in each case. In all reconstructions we only show the ROI, and we indicate the RMSE in traveltimes. The initial RMSE is $1.77 \cdot 10^{-6} s$.

We apply the D-SOED method to jointly optimize the emitter configurations for transmission and reflection, using both straight-ray and finite-frequency transmission techniques. As in the example before, we parameterize the model using a rectilinear grid with $1 \mathrm{~mm}$ mesh size. The benefit-cost curves are shown in Figure 7.5(a). In both curves the optimal benefit-cost ratio is reached for 4 emitters. This may be an effect of the relatively high frequencies used in the experiment, in which the finite-frequency sensitivities become closer to ray-based predictions. For a fixed number of emitters, the curves moreover show how the information gain varies for randomly selected emitter locations. In this application, the choice of the emitter locations has a less significant effect on the information gain than 
the number of emitters. This is due to the good data coverage provided by the aperture, in general, and by not reducing the number of receivers during the D-SOED, in particular. Despite the small variations, our D-SOED algorithm certainly selects solutions close to the optimal in each case.

To verify our interpretation of the benefit-cost curves, we compare the reconstructions obtained from the optimized setups with the ones obtained using all emitters. For transmission tomography, we apply total variation regularization (Jensen et al., 2012), and the results are shown in Figures 7.5(b) - 7.5(e). Our reconstructions are in agreement with the results obtained by other studies (Ruiter et al., 2018). For both imaging techniques, reconstructions using only $25 \%$ of the dataset are almost identical to the ones using the complete dataset. The root-mean-square error (RMSE), comparing the observed traveltimes with the traveltimes predicted by the reconstruction, is indicated in the figures. In all cases, we compute the RMSE using the complete dataset, showing that the results from optimized setups explain the data equally well. This means that, effectively, our D-optimal algorithm accurately identifies the redundancies in the measurements. To validate the information encoded by the benefit-cost curves, we moreover show in Figure 7.6 the reconstructions at different stages of the curve corresponding to finite-frequency tomography. We compare reconstructions using 2, 3 and 4 optimally selected emitters. These results demonstrate that our estimation of the information gain certainly captures the amount of relative information each experimental design is expected to provide about the ROI.
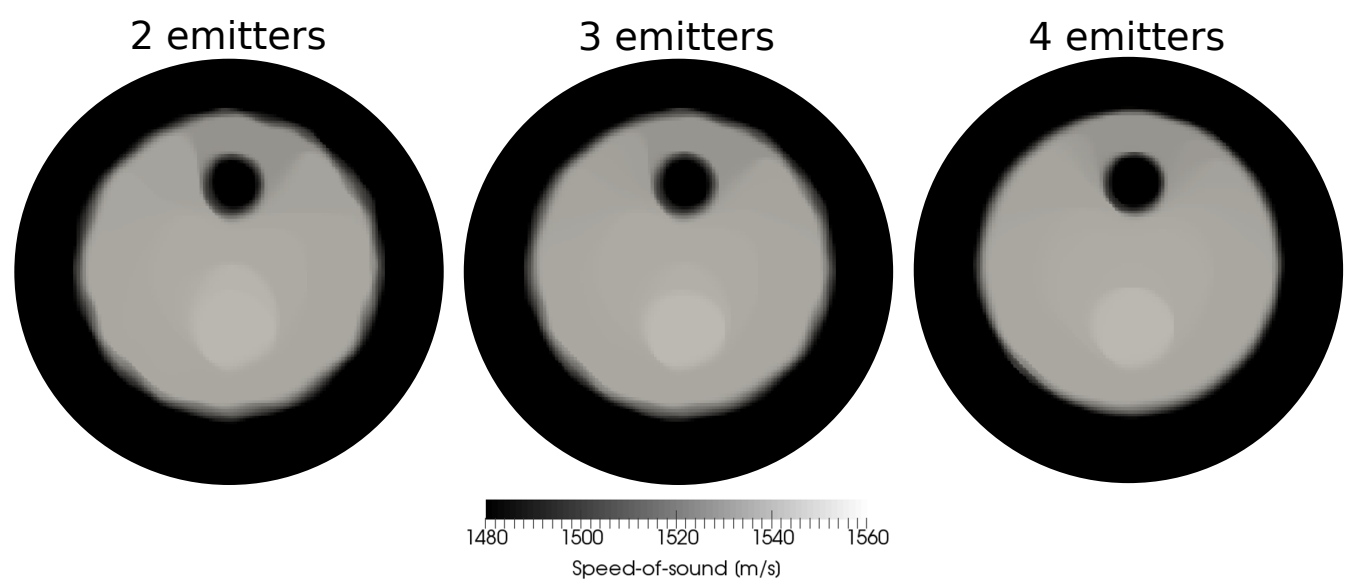

Figure 7.6.: Velocity reconstructions using traveltime finite-frequency tomography. (From left to right) The number of optimally selected emitters are 2, 3 and 4, respectively.

Because the emitter configuration is simultaneously optimized also for reflectivity imaging techniques, in Figure 7.7 we show the images resulting from B-mode and RTM techniques. We use the spectral element solver Salvus (Afanasiev et al., 2019) for the numerical wave propagation simulations required for RTM. Similar to the transmission case, there are 


\section{Designing USCT experiments: Part II}

no significant differences between the images obtained from 4 and 16 emitters. In all cases, the features resolved with the complete dataset are also recovered when using only $25 \%$ of the dataset. Here, moreover, we do not only verify the efficiency of the D-optimal design algorithm, but we also validate the linearization assumptions made in the context of optimal experimental design. Although we select the most informative emitters assuming a homogeneous model in the estimation of the covariance operator, the reflectivity images are obtained using velocity reconstructions shown in Figure 7.5.
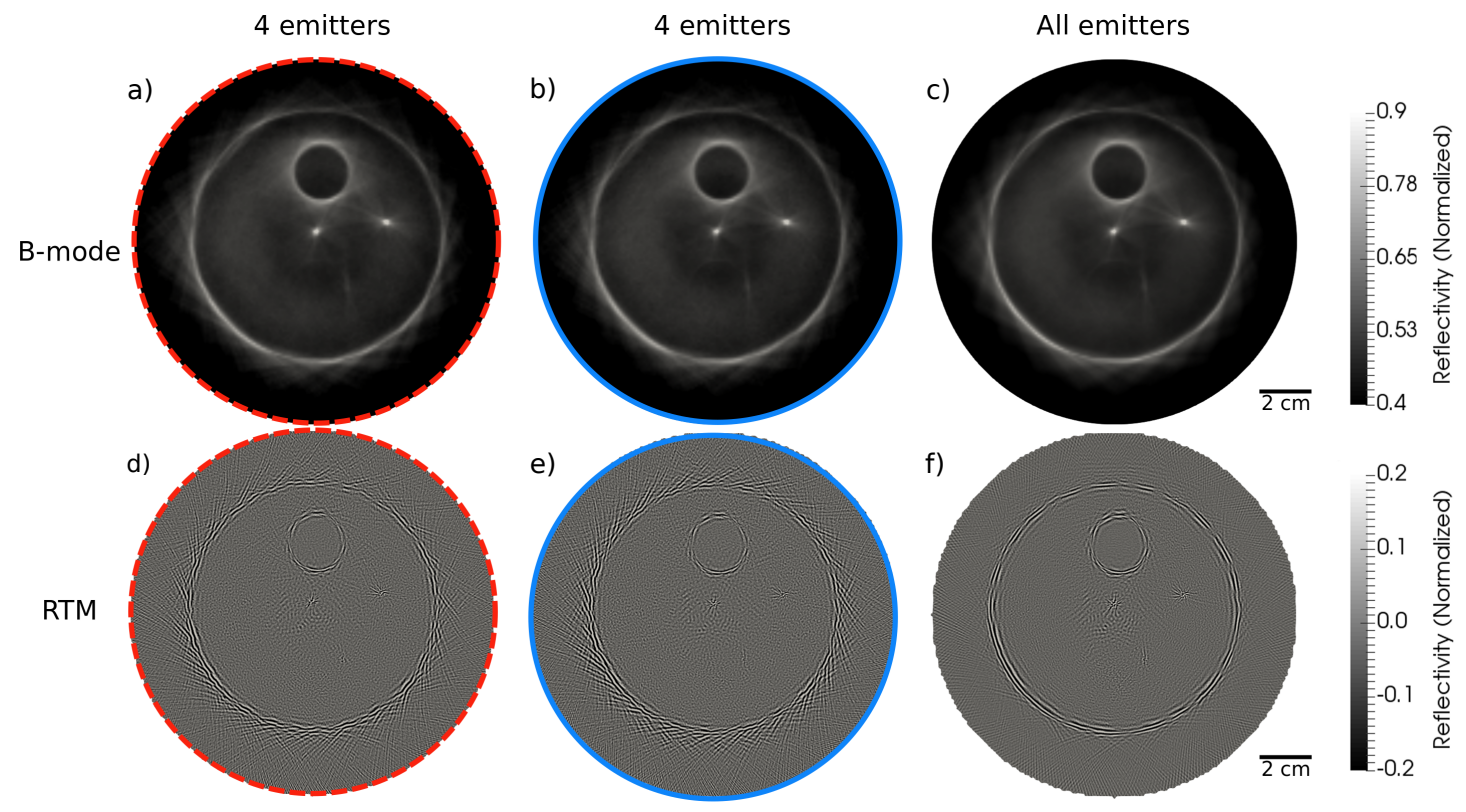

Figure 7.7.: Reflectivity images using B-mode technique (top row) and RTM (bottom row). The emitter configurations used are: (left column) optimally selected 4 emitters jointly with straight-ray transmission; (middle column) 4 emitters jointly selected with finite-frequency transmission; and (right column) all emitters. Red and blue circles indicate the background velocity models in Figure 7.5 used for reflectivity imaging.

\subsection{Discussion and Conclusion}

In this study, we introduce the D-SOED method in the context of breast tissue imaging with ultrasound. Our approach is flexible enough to optimize the acquisition system for both transmission and reflection data, and using either ray-based or wave-based methods for the reconstructions. This flexibility is crucial for USCT, where the final product is a collection of images of different acoustic properties of the tissue. Although we only consider reflectivity and velocity, the extension to other properties, such as attenuation and density, is straightforward. The performance comparison for different imaging methods is beyond 
the scope of this work.

The concept of optimality however is ambiguous, and this can be observed, for instance, in the number of different optimality criteria available in the literature. This ambiguity is caused by the constraint of expressing with a scalar value the information contained in the covariance operator. Typically, one must choose which statistical properties will be emphasized at the expenses of the rest, and this selection depends on the aim of the experiment itself. In our case, we define the optimal experimental design through the Doptimality condition, which minimizes the volume of the joint confidence ellipsoid for the estimated parameters. Therefore, our results do not necessarily guarantee to improve other attributes, as for instance the inter-parameter trade-offs. A more integrating approach could be based on the combination of different optimality criteria (Curtis, 1999b).

Additional constraints introduced in the optimal experimental design method, also include the definition of the design parameters. Here, we choose to select the number and locations of the informative transducers, but other choices are also possible. To reduce the space of the possible experimental designs, we predefine some candidate values for the design parameters. This requires some prior information about the acquisition system, which typically is available. Examples of this are the approximate dimensions of the transducer holder or the frequencies of the emitted signal. Note that all these choices will affect the optimal design, which is important to keep in mind when interpreting the results. Under these constraints, however, the D-optimality condition has an important invariant property that makes our results general. Different model parameterizations yield same D-optimal designs. This is crucial when the parameterization is not fixed for post-acquisition reconstructions.

Although ideally the experimental design should be optimized prior to any realization of the experiment, we demonstrate that our approach can similarly be applied post-acquisition. In this way, the computational cost of the reconstructions can be controlled, and this has a significant impact in the context of clinical practice, where fast answers are key. Similar approaches have recently been applied in geophysical exploration to reduce the large data volume in marine seismic surveys (Coles et al., 2015). However, the redundancies identified by the D-SOED approach may also be used not just to reduce the dataset but as a complementary analysis for other methods. These could include, for example, the mini-batch (Boehm et al., 2018) or source encoding (Wang et al., 2015) approaches in the context of waveform tomography.

Finally, it is important to mention that, while the computational cost of D-SOED method may be challenging for large-scale problems, the benefit of an optimized USCT system, in terms of the image quality and computational resources, for clinical practice is tremendous. Once the acquisition system is optimized, the impact of the reduced cost in the recursive use of the scanning system may become very significant. The results presented in this chapter show that high quality images can be obtained reducing four times the computational resources needed per each realization of the experiment. 


\subsection{Outlook: Sensitivity of the quality measure}

The derivatives of the quality measure $\Theta$ with respect to design parameters $\mathbf{s}$ can provide useful information about the sensitivity of each design parameter $s_{i}$ to the uncertainties in the estimated model parameters. Assume we relax the binary condition of the entries of $\mathbf{s}$ allowing $s_{i} \in[0,1]$ for any $i=1, \ldots, N_{s}$, with $N_{s}$ being the number of candidate transducer locations. In principle, non-binary values are difficult to interpret for transducer locations. A possible approach could be to transform them back into a binary design by adopting some sort of thresholding (e.g., Attia et al., 2018). Assume moreover that we express the forward problem as

$$
\mathbf{d}_{\mathrm{s}}=\mathbf{F}(\mathbf{s}, \mathbf{m})=\mathbf{S F}(\mathbf{m}),
$$

where $\mathbf{S}=\operatorname{diag}\left(s_{1}, \ldots, s_{N_{s}}\right), \mathbf{F}$ refers to the comprehensive experiment in which $\mathbf{s}=$ $(1, \ldots, 1)$, and $\mathbf{d}_{\mathbf{s}}$ denotes the observations related to the selected experimental setup $\mathbf{S}$. As usual, we linearize Equation (7.5) as

$$
\delta \mathbf{d}_{\mathrm{s}}=\mathbf{S F}^{\prime} \delta \mathbf{m}
$$

for which the posterior covariance operator is analytically given by

$$
\boldsymbol{\Gamma}_{\text {post }}=\mathbf{H}^{-1}=\left(\mathbf{F}^{\prime T} \mathbf{S} \boldsymbol{\Gamma}_{\text {noise }}^{-1} \mathbf{S F}^{\prime}+\boldsymbol{\Gamma}_{\text {prior }}^{-1}\right)^{-1} .
$$

From here, we can derive the gradient of the D-optimality condition with respect to $\mathbf{s}$ if we observe that

$$
\nabla_{\mathbf{s}} \Theta(\mathbf{s})=\sum_{i=1}^{N_{s}} \frac{\partial\left(\log \operatorname{det} \boldsymbol{\Gamma}_{\text {post }}\right)}{\partial s_{i}} \mathbf{e}_{i}=\sum_{i=1}^{N_{s}} \operatorname{trace}\left(\boldsymbol{\Gamma}_{\text {post }}^{-1} \frac{\partial \boldsymbol{\Gamma}_{\text {post }}}{\partial s_{i}}\right) \mathbf{e}_{i},
$$

where $\mathbf{e}_{i}$ is the $i$-th coordinate vector in $\mathbb{R}^{N_{s}}$. Here, we closely follow similar derivations found in Attia et al. (2018) and Attia and Constantinescu (2018). Using the definition of $\boldsymbol{\Gamma}_{\text {post }}$ in Equation (7.7), where $\mathbf{H}_{\text {misfit }}$ refers to its first term, it follows that

$$
\begin{aligned}
\operatorname{trace} & \left(\boldsymbol{\Gamma}_{\text {post }}^{-1} \frac{\partial \boldsymbol{\Gamma}_{\text {post }}}{\partial s_{i}}\right)=-\operatorname{trace}\left(\frac{\partial \mathbf{H}_{\text {misfit }}}{\partial s_{i}} \boldsymbol{\Gamma}_{\text {post }}\right) \\
& =-\operatorname{trace}\left(\mathbf{F}^{\prime T} \frac{\partial \mathbf{S}}{\partial s_{i}} \boldsymbol{\Gamma}_{\text {noise }}^{-1} \mathbf{S} \mathbf{F}^{\prime} \boldsymbol{\Gamma}_{\text {post }}\right)-\operatorname{trace}\left(\mathbf{F}^{\prime T} \mathbf{S} \boldsymbol{\Gamma}_{\text {noise }}^{-1} \frac{\partial \mathbf{S}}{\partial s_{i}} \mathbf{F}^{\prime} \boldsymbol{\Gamma}_{\text {post }}\right) \\
& =-2 \operatorname{trace}\left(\mathbf{F}^{\prime T} \mathbf{S} \boldsymbol{\Gamma}_{\text {noise }}^{-1} \frac{\partial \mathbf{S}}{\partial s_{i}} \mathbf{F}^{\prime} \boldsymbol{\Gamma}_{\text {post }}\right),
\end{aligned}
$$

where we use the forward operator definition in Equation (7.6), and we simplify the expression in the last step by applying properties of the trace. We can express the design 
matrix as

$$
\mathbf{S}=\sum_{i=1}^{N_{s}} s_{i} \mathbf{e}_{i} \mathbf{e}_{i}^{T}
$$

and we obtain

$$
\frac{\partial \mathbf{S}}{\partial s_{i}}=\mathbf{e}_{i} \mathbf{e}_{i}^{T}
$$

Upon inserting this into Equation (7.9) and using the cyclic property of the trace operator, we can rewrite

$$
\begin{aligned}
\operatorname{trace}\left(\boldsymbol{\Gamma}_{\text {post }}^{-1} \frac{\partial \boldsymbol{\Gamma}_{\text {post }}}{\partial s_{i}}\right) & =-2 \operatorname{trace}\left(\mathbf{F}^{\prime T} \mathbf{S} \boldsymbol{\Gamma}_{\text {noise }}^{-1} \mathbf{e}_{i} \mathbf{e}_{i}^{T} \mathbf{F}^{\prime} \boldsymbol{\Gamma}_{\text {post }}\right) \\
& =-2 \operatorname{trace}\left(\mathbf{e}_{i}^{T} \mathbf{F}^{\prime} \boldsymbol{\Gamma}_{\text {post }} \mathbf{F}^{\prime T} \mathbf{S} \boldsymbol{\Gamma}_{\text {noise }}^{-1} \mathbf{e}_{i}\right) \\
& =-2\left(\mathbf{e}_{i}^{T} \mathbf{F}^{\prime} \boldsymbol{\Gamma}_{\text {post }} \mathbf{F}^{\prime T} \mathbf{S} \boldsymbol{\Gamma}_{\text {noise }}^{-1} \mathbf{e}_{i}\right) .
\end{aligned}
$$

Moreover, if we assume that the noise covariance is $\boldsymbol{\Gamma}_{\text {noise }}=\sigma_{n} \mathbf{I}$, the gradient in Equation (7.8) becomes

$$
\nabla_{\mathbf{s}} \Theta(\mathbf{s})=-2 \sigma_{n}^{-1} \sum_{i=1}^{N_{s}} s_{i}\left(\mathbf{e}_{i}^{T} \mathbf{F}^{\prime} \boldsymbol{\Gamma}_{\text {post }} \mathbf{F}^{T} \mathbf{e}_{i}\right) \mathbf{e}_{i} .
$$

This equation gives the analytical expression of the sensitivities of design parameters to the quality measure.

To understand the information that is encoded in $\nabla_{\mathbf{s}} \Theta(\mathbf{s})$, we consider the toy example in Section 7.2. Starting from a comprehensive experiment that considers all candidate receivers active, i.e., a total of 124 receivers, we compute the sensitivity of the quality measure to each receiver location. We assign a number to each receiver, 1 being next to the emitter location and increasing the numbers in clockwise direction. Figure 7.8(a) shows the corresponding gradients for the three imaging techniques considered in Section 7.2. Here, we drop the -2 term from Equation (7.11) for a more intuitive understanding of the curves, i.e., we focus in its absolute and normalized values. These results clearly show what we already observed in Section 7.2.2: (1) comparing straight-ray and finite-frequency transmission, we observe that more receivers in transmission-side are sensitive to the quality measure computed from finite-frequency considerations; and (2) the most informative (sensitive) receivers for straight-ray transmission are the least informative (sensitive) ones for reflection. Furthermore, these gradients also provide new insights: receivers in the transmission-side are the most sensitive ones to the quality measure computed for reflection. This may be a consequence of constraining larger number of model parameters by these receivers than the ones in reflection-side [see Figure 7.1]. In any case, further studies are needed to understand if this is a general feature or, on the contrary, particular to this toy example.

To validate the analytical expression for the gradient in Equation (7.11), we take the 


\section{Designing USCT experiments: Part II}

(a)

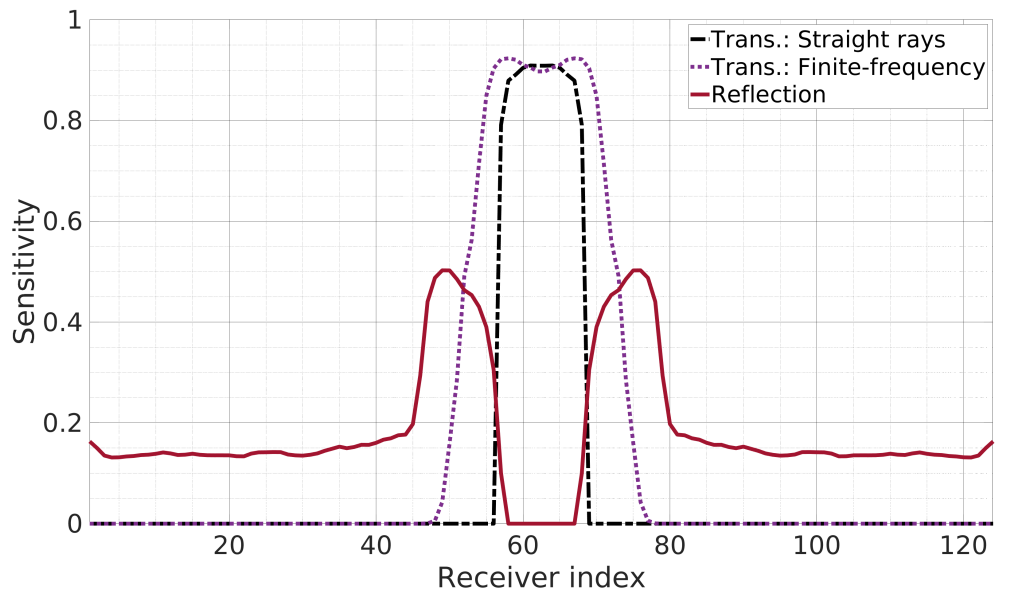

(b)

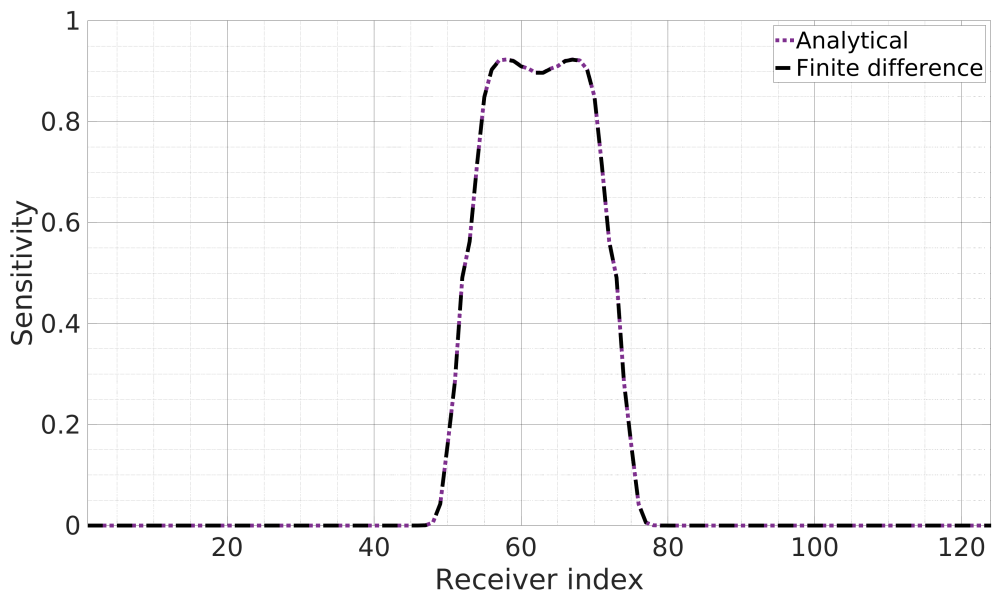

Figure 7.8.: (a) The gradient of the quality measure $\Theta$ with respect to $\mathrm{s}$ for the toy example in Section 7.2, where we consider straight-ray and finite-frequency transmission, and reflection imaging techniques. (b) Validation of the analytical gradient with finite-difference approach.

example of the finite-frequency transmission and compute the gradient using the finitedifference approach:

$$
\delta \mathbf{s}^{T} \nabla_{\mathbf{s}} \Theta(\mathbf{s})=\lim _{\epsilon \rightarrow 0} \frac{\Theta(\mathbf{s}+\epsilon \delta \mathbf{s})-\Theta(\mathbf{s})}{\epsilon} .
$$

We can compute each individual component of the gradient by properly choosing perturbation directions for design parameters $\delta \mathbf{s}$. For instance, direction $\delta \mathbf{s}_{i}=\left(0, \ldots, s_{i}=1, \ldots, 0\right)$ will provide the component $\frac{\partial \Theta(\mathrm{s})}{\partial s_{i}}$. Figure 7.8(b) shows a comparison of the gradient computed from both the analytical and finite-difference approach. This result validates our derivation of the analytical expression in Equation (7.11).

Similar expressions can be derived for different quality measures. For example, Attia et al. (2018) and Attia and Constantinescu (2018) provide the gradient of the A-optimality 
condition and use this to optimize the experimental design by applying gradient-based optimization algorithms. This can be seen as an alternative to SOED approaches, and it will require sparsifying penalty functions to ensure that the optimal designs consist of a limited number of active transducers. Finally, similar derivations can also be used to derive the second-order derivatives of $\Theta$ with respect to $\mathbf{s}$, which encode important information about trade-offs between design parameters. This can be useful to improve our understanding about the experimental design. 



\section{Discussion and final remarks}

"The science question in feminism is about objectivity as positioned rationality. Its images are not the products of escape and transcendence of limits (the view from above) but the joining of partial views and halting voices into a collective subject position that promises a vision of the means of ongoing finite embodiment, of living within limits and contradictions - of views from somewhere."

- Donna Haraway, Situated Knowledges (1988)

The thesis presented here is a response to recent efforts in resuming cross-fertilization processes between seismology and USCT. While both disciplines have been closely connected since their origins, interdisciplinary studies have not been continuous in time and mostly appear when specific needs request them. Our context is not different. Recent developments in computational and hardware technologies have facilitated the revival of research in USCT for breast imaging. To be competitive in current times, USCT requires sophisticated imaging techniques capable for early-stage breast cancer detection. In this situation, the pioneering work of Pratt et al. (2007), in transferring 2D FWI from seismic exploration to USCT, has stimulated the emergence of multiple interdisciplinary projects and spaces within the last decade. This thesis is one of them and, therefore, not unique in its intentions.

\subsection{From seismology to USCT}

There are several other works that have focused on transferring technologies and knowledge from seismology to medical ultrasound. In the following, we describe some of their most important contributions and connect them with the open questions in USCT that were listed at the end of Chapter 3 .

1. Calderón Agudo et al. (2018) have studied the extension of FWI to 3D domains and analyzed the impact of low-frequency transducers or good starting models in the accuracy of the reconstructions. Their results demonstrate the need for 3D and low-frequency data $(\sim 300 \mathrm{kHz})$ to ensure accurate and high-fidelity reconstructions, as well as to enable robust and automatic inversions. They do not specifically address any of our listed open questions, but their results definitely show (1) the importance 


\section{Discussion and final remarks}

of adapting existing acquisition systems to new imaging techniques, and (2) the need for $3 \mathrm{D}$ reconstructions.

2. Taskin et al. (2018) have introduced the redatuming technique to USCT to reduce the computational cost of full-waveform inversion. This scales with the size of the spatial domain in which waves propagate, and thus, their approach consist in constraining the numerical wave propagation simulations to a smaller domain. The method has been successfully applied in seismic exploration and allows to back-propagate the measured wavefield to virtual receiver locations that are closer to the ROI. Their results are promising for numerical and experimental data in $2 \mathrm{D}$, showing that the new approach may provide significant computational savings for FWI applications in USCT.

3. Following a similar goal, Boehm et al. (2018) have combined ideas from large-scale nonlinear optimization, machine learning and global-scale seismology to reduce the computational cost of 3D FWI in USCT. Using goal-oriented meshing and spectralelement waveform simulations, the computational domain is truncated to the transducer coverage of the scanning device. Furthermore, they propose a dynamic minibatch approach to exploit redundancies in the measurements and introduce a stochastic trust-region L-BFGS inversion scheme, that randomly selects subsets of emitters in each iteration.

As we observe, these studies closely follow the path introduced by Pratt et al. (2007) and are mainly concerned with reducing the cost of waveform tomography. Therefore, they cover aspects that complement the ones addressed in this thesis with regard to the questions listed in Chapter 3. Our work, however, goes beyond waveform tomography. We take the opportunity offered by interdisciplinary perspectives to bring and explore other approaches useful for USCT. Consequently, we contribute to diversify the possibilities, including methods to optimally design scanning devices and finite-frequency tomography.

\subsection{Specific contributions}

This thesis makes valuable contributions specific to USCT that, to the best of our knowledge, are unique to this field and may serve for future advances in breast ultrasound tomography. These include, in order of appearance within the thesis:

1. Traveltime tomography that considers finite-frequency sensitivities, particularly suited to obtain physically consistent 3D reconstructions using slice-by-slice acquisition systems. We furthermore present an extension to amplitude tomography for inferring velocity distribution of breast tissue. 
2. A resolution analysis approach using estimations of point-spread functions for transmission tomography, particularly useful to evaluate the performance of USCT scanning systems.

3. A systematic framework to optimally design USCT experiments that accounts for multimodality imaging and finds compromises between the post-acquisition image quality and computational cost.

The latter includes moreover innovative extensions that are unusual for optimal experimental design studies in geophysical applications. For instance, (1) we solve large-scale problems with 3D emitter-receiver configurations, (2) we compare solutions obtained by applying global and sequential approaches to the same problem, and (3) we present a joint optimal experimental design approach that simultaneously optimizes the experiment for multiple imaging techniques and parameters.

\subsection{Cross-fertilization: back to seismology?}

One may ask whether there is any benefit for seismology resulting from these collaborative projects with USCT. The answer is definitely yes. It would be misleading to think of this exchange in hierarchical terms, where each field has either an active or passive role. In fact, USCT experiments may to a certain extent represent an analogue experiment for seismic tomography, providing laboratory models under controlled conditions useful to verify tomography techniques across different scales. In the following, we discuss several approaches studied in USCT that either have been or could be transferred to seismic applications.

While stochastic optimization strategies using subsets of emitters is not new for seismology (van Leeuwen and Herrmann, 2013), the dynamic mini-batch approach suggested by Boehm et al. (2018) has recently been transferred, extended and tailored to continentalscale seismic tomography (van Herwaarden et al., 2019). To control and minimize redundancies in the selected mini-batch of events, their work additionally includes strategies introduced by Vinard et al. (2018) for SOED of USCT devices. In this specific case, the exchange between both fields has stimulated the development of an evolutionary framework for seismic full-waveform inversion that (1) leads to significant computational savings, thereby potentially enabling more tractable global-scale applications, and (2) offers the possibility to incorporate new earthquake data without the need of reinverting the complete dataset (van Herwaarden et al., 2019).

This thesis also makes specific contributions that can find multiple applications in seismic tomography. For instance, the knowledge acquired from optimal experimental design methods can potentially be transferred back to other applications that go beyond traditional seismic exploration. An example of this we find in ambient seismic source inversion, which 


\section{Discussion and final remarks}

uses seismic noise recordings from all available station pairs to image the noise source distribution at global or regional scales (e.g., Nishida and Fukao, 2007; Ermert et al., 2017). The resulting source models are useful for the investigation of ocean microseism sources (e.g., Stutzmann et al., 2012) and for ambient noise tomography (e.g., Sager et al., 2017). Because the technique is based on interstation correlations, data coverage is completely controlled by seismic station locations. Currently, dense station networks are becoming increasingly available (e.g., Nakata et al., 2019), which provide large amounts of data. These are well suited to ambient noise applications, but may be dominated by redundant information. Due to the linear relationship between cross correlations of noise recordings and noise sources, optimal experimental design methods studied in Chapters 6 and 7 can offer a valuable tool to identify the most informative stations, thereby alleviating the imaging process without compromising the outcome. Similar ideas can also be extended to increase the general understanding about optimal array configurations for ambient seismic noise studies (Maranò et al., 2014; Kennett et al., 2015).

These are examples that show the value of interdisciplinary collaborations. They illustrate the potential of looking at the same object of study from different perspectives. Creative or innovative answers may appear in this process, which is in essence a collective one, i.e., the result of the communication between two disciplines and their communities. This thesis wants to stay in their intersection, never fully belonging to any one. 


\section{Born approximation}

In this appendix we derive the Born approximation of the acoustic wave equation for loss-less media and constant density. In this case, the pressure field $p(\mathbf{x}, t)$ satisfies the wave equation

$$
\frac{1}{c^{2}(\mathbf{x})} \partial_{t}^{2} p(\mathbf{x}, t)-\nabla^{2} p(\mathbf{x}, t)=f\left(\mathbf{x}_{s}, t\right)
$$

and the corresponding Green's function is given by

$$
\frac{1}{c^{2}(\mathbf{x})} \partial_{t}^{2} G\left(\mathbf{x}, t ; \mathbf{x}^{\prime}, t^{\prime}\right)-\nabla^{2} G\left(\mathbf{x}, t ; \mathbf{x}^{\prime}, t^{\prime}\right)=\delta\left(\mathbf{x}-\mathbf{x}^{\prime}\right) \delta\left(t-t^{\prime}\right),
$$

which describes the response of the medium due to a point-localized delta-pulse source. Green's functions are useful quantities that allow us to express the solution to Equation (A.1) as

$$
p(\mathbf{x}, t)=\int_{V} \int_{T} G\left(\mathbf{x}, t ; \mathbf{x}^{\prime}, t^{\prime}\right) f\left(\mathbf{x}^{\prime}, t^{\prime}\right) d t^{\prime} d \mathbf{x}^{\prime}
$$

where $\mathrm{V}$ is the volume of our domain of interest and $\mathrm{T}$ is the time interval of interest. This is commonly known as the representation theorem (Aki and Richards, 2002).

Now, let us assume that we introduce a small perturbation in the velocity $c(\mathbf{x}) \rightarrow$ $c(\mathbf{x})+\delta c(\mathbf{x})$, which produces a perturbation in the pressure field $p(\mathbf{x}, t) \rightarrow p(\mathbf{x}, t)+\delta p(\mathbf{x}, t)$. The new wavefield will satisfy the wave equation

$$
\frac{1}{[c(\mathbf{x})+\delta c(\mathbf{x})]^{2}} \partial_{t}^{2}[p(\mathbf{x}, t)+\delta p(\mathbf{x}, t)]-\nabla^{2}[p(\mathbf{x}, t)+\delta p(\mathbf{x}, t)]=f\left(\mathbf{x}_{s}, t\right) .
$$

If the velocity anomaly is small compared to the background velocity, we can use Taylor expansion to approximate the coefficient of the first term as

$$
\frac{1}{[c(\mathbf{x})+\delta c(\mathbf{x})]^{2}} \approx \frac{1}{c^{2}(\mathbf{x})}-\frac{2 \delta c(\mathbf{x})}{c^{3}(\mathbf{x})}
$$

where higher order perturbations have been neglected. Upon inserting Equation (A.5) into Equation (A.7), we obtain

$$
\frac{1}{c^{2}(\mathbf{x})} \partial_{t}^{2}[p(\mathbf{x}, t)+\delta p(\mathbf{x}, t)]-\frac{2 \delta c(\mathbf{x})}{c^{3}(\mathbf{x})} \partial_{t}^{2}[p(\mathbf{x}, t)+\delta p(\mathbf{x}, t)]-\nabla^{2}[p(\mathbf{x}, t)+\delta p(\mathbf{x}, t)]=f\left(\mathbf{x}_{s}, t\right),
$$




\section{A. Born approximation}

and using the equivalence in Equation (A.1),

$$
\frac{1}{c^{2}(\mathbf{x})} \partial_{t}^{2} \delta p(\mathbf{x}, t)-\nabla^{2} \delta p(\mathbf{x}, t)=\frac{2 \delta c(\mathbf{x})}{c^{3}(\mathbf{x})} \partial_{t}^{2} p(\mathbf{x}, t)+\frac{2 \delta c(\mathbf{x})}{c^{3}(\mathbf{x})} \partial_{t}^{2} \delta p(\mathbf{x}, t) .
$$

This equation shows that the scattered wavefield $\delta p(\mathbf{x}, t)$ is generated due to two new source terms: the first term on the right-hand side accounts for the interaction of the unperturbed pressure field $p(\mathbf{x}, t)$ with velocity anomalies $c(\mathbf{x})$ and defines the first-order scattering effects. The second term, on the other hand, describes second-order scattering effects, i.e., the interaction of the perturbed wavefield with the velocity anomalies. These effects are often negligible, particularly when the medium shows relatively low variations, and once neglected, the approach is referred as the Born approximation. In such situations, the wave equation for the scattered wavefield becomes

$$
\left(\frac{1}{c^{2}(\mathbf{x})} \partial_{t}^{2}-\nabla^{2}\right) \delta p(\mathbf{x}, t)=\frac{2 \delta c(\mathbf{x})}{c^{3}(\mathbf{x})} \partial_{t}^{2} p(\mathbf{x}, t)
$$

As we observe, the left-hand side of the wave equation is very similar to the one in Equation (A.1) or (A.2), that is, the medium in which the scattered field propagates remains the same. Consequently, we can express $\delta p(\mathbf{x}, t)$ in terms of Green's function of the background medium $c(\mathbf{x})$, similar to Equation (A.3), as

$$
\delta p(\mathbf{x}, t)=\int_{V} \int_{T} G\left(\mathbf{x}, t ; \mathbf{x}^{\prime}, t^{\prime}\right) \frac{2 \delta c\left(\mathbf{x}^{\prime}\right)}{c^{3}\left(\mathbf{x}^{\prime}\right)} \partial_{t^{\prime}}^{2} p\left(\mathbf{x}^{\prime}, t^{\prime}\right) d t^{\prime} d \mathbf{x}^{\prime}
$$

and reformulating it we obtain

$$
\delta p(\mathbf{x}, t)=\int_{V^{\prime}} \int_{V^{\prime \prime}} \frac{2 \delta c\left(\mathbf{x}^{\prime}\right)}{c^{3}\left(\mathbf{x}^{\prime}\right)} G\left(\mathbf{x}, t ; \mathbf{x}^{\prime}, 0\right) * G\left(\mathbf{x}^{\prime}, t ; \mathbf{x}^{\prime \prime}, 0\right) * \partial_{t}^{2} f\left(\mathbf{x}^{\prime \prime}, t\right) d \mathbf{x}^{\prime} d \mathbf{x}^{\prime \prime},
$$

where ${ }^{*}$ denotes time convolution. Equation (A.10) is similar to the one obtained for reverse-time migration in Section 4.1.2. 


\section{Low-rank approximation}

This appendix derives the low-rank approximations of the posterior covariance $\boldsymbol{\Gamma}_{\text {post }}$ and resolution matrix $\mathbf{R}$ introduced in Section 4.2.5.

\section{B.1. Posterior covariance matrix}

We observed that the posterior covariance can be expressed in terms of the priorpreconditioned data-misfit Hessian $\widetilde{\mathbf{H}}_{\text {misfit }}$ as

$$
\boldsymbol{\Gamma}_{\text {post }}=\left(\mathbf{F}^{\prime T} \boldsymbol{\Gamma}_{\text {noise }}^{-1} \mathbf{F}^{\prime}+\boldsymbol{\Gamma}_{\text {prior }}^{-1}\right)^{-1}=\boldsymbol{\Gamma}_{\text {prior }}^{1 / 2}\left(\widetilde{\mathbf{H}}_{\text {misfit }}+\mathbf{I}\right)^{-1} \boldsymbol{\Gamma}_{\text {prior }}^{1 / 2},
$$

where

$$
\widetilde{\mathbf{H}}_{\text {misfit }}:=\boldsymbol{\Gamma}_{\text {prior }}^{1 / 2} \mathbf{F}^{\prime T} \boldsymbol{\Gamma}_{\text {noise }}^{-1} \mathbf{F}^{\prime} \boldsymbol{\Gamma}_{\text {prior }}^{1 / 2} .
$$

By applying SVD on $\widetilde{\mathbf{H}}_{\text {misfit, }}$, we can approximate it by retaining the largest $k<N$ eigenvalues and the corresponding eigenvectors, i.e.,

$$
\widetilde{\mathbf{H}}_{\text {misfit }}=\mathbf{V} \boldsymbol{\Lambda} \mathbf{V}^{T} \approx \mathbf{V}_{k} \boldsymbol{\Lambda}_{k} \mathbf{V}_{k}^{T},
$$

where $\boldsymbol{\Lambda}=\operatorname{diag}\left(\lambda_{i}\right) \in \mathbb{R}^{N \times N}$ and $\mathbf{V} \in \mathbb{R}^{N \times N}$ are the eigenvalue and eigenvector matrix, respectively. Here, $\boldsymbol{\Lambda}_{k} \in \mathbb{R}^{k \times k}$ and $\mathbf{V}_{k} \in \mathbb{R}^{N \times k}$ represent the truncated eigenvalue and eigenvector matrices.

For the moment, we forget the approximation in Equation (B.3), and we insert this into Equation (B.1) to obtain

$$
\boldsymbol{\Gamma}_{\text {post }}=\boldsymbol{\Gamma}_{\text {prior }}^{1 / 2}\left(\mathbf{V} \boldsymbol{\Lambda} \mathbf{V}^{T}+\mathbf{I}\right)^{-1} \boldsymbol{\Gamma}_{\text {prior }}^{1 / 2}=\boldsymbol{\Gamma}_{\text {prior }}^{1 / 2}\left(\mathbf{V} \boldsymbol{\Lambda} \mathbf{V}^{T}+\mathbf{I}\right)^{-1} \boldsymbol{\Gamma}_{\text {prior }}^{1 / 2}
$$

Here, we can compute the inverse of the term in the middle by applying the ShermanMorrison-Woodbury formula (e.g., Hager, 1989), which states that

$$
\left(\mathbf{A}+\mathbf{X} \mathbf{Y}^{T}\right)^{-1}=\mathbf{A}^{-1}-\mathbf{A}^{-1} \mathbf{X}\left(\mathbf{I}+\mathbf{Y}^{T} \mathbf{A}^{-1} \mathbf{X}\right)^{-1} \mathbf{Y}^{T} \mathbf{A}^{-1}
$$


Using this formula in Equation (B.8), we obtain

$$
\left(\mathbf{V} \Lambda \mathbf{V}^{T}+\mathbf{I}\right)^{-1}=\mathbf{I}-\mathbf{V} \boldsymbol{\Lambda}(\mathbf{I}+\mathbf{\Lambda})^{-1} \mathbf{V}^{T}=\mathbf{I}-\mathbf{V D V}^{T}
$$

where $\mathbf{D}=\operatorname{diag}\left(\frac{\lambda_{i}}{\lambda_{i}+1}\right)$. Here, we can apply the low-rank approximation, which gives

$$
\left(\mathbf{V} \boldsymbol{\Lambda} \mathbf{V}^{T}+\mathbf{I}\right)^{-1}=\mathbf{I}-\mathbf{V}_{k} \mathbf{D}_{k} \mathbf{V}_{k}^{T}+\mathcal{O}\left(\sum_{i=k+1}^{N} \frac{\lambda_{i}}{\lambda_{i}+1}\right) \approx \mathbf{I}-\mathbf{V}_{k} \mathbf{D}_{k} \mathbf{V}_{k}^{T}
$$

This equation suggests that we obtain a good approximation by truncating eigenvalues that satisfy $\lambda_{i} \ll 1$. Finally, upon inserting Equation (B.7) into (B.7) we obtain the low-rank approximation of the posterior covariance matrix,

$$
\boldsymbol{\Gamma}_{\text {post }} \approx \boldsymbol{\Gamma}_{\text {prior }}^{1 / 2}\left(\mathbf{I}-\mathbf{V}_{k} \mathbf{D}_{k} \mathbf{V}_{k}^{T}\right) \boldsymbol{\Gamma}_{\text {prior }}^{1 / 2}=\boldsymbol{\Gamma}_{\text {prior }}-\boldsymbol{\Gamma}_{\text {prior }}^{1 / 2} \mathbf{V}_{k} \mathbf{D}_{k} \mathbf{V}_{k}^{T} \boldsymbol{\Gamma}_{\text {prior }}^{1 / 2}
$$

\section{B.2. Resolution matrix}

In similar way, we can derive the low-rank approximation for the resolution matrix

$$
\mathbf{R}=\left(\mathbf{F}^{T} \boldsymbol{\Gamma}_{\text {noise }}^{-1} \mathbf{F}+\boldsymbol{\Gamma}_{\text {prior }}^{-1}\right)^{-1} \mathbf{F}^{T} \boldsymbol{\Gamma}_{\text {noise }}^{-1} \mathbf{F}=\boldsymbol{\Gamma}_{\text {post }} \mathbf{F}^{T} \boldsymbol{\Gamma}_{\text {noise }}^{-1} \mathbf{F},
$$

i.e.,

$$
\begin{aligned}
\mathbf{R} & =\boldsymbol{\Gamma}_{\text {prior }}^{1 / 2}\left(\mathbf{I}-\mathbf{V} \mathbf{D} \mathbf{V}^{T}\right) \boldsymbol{\Gamma}_{\text {prior }}^{1 / 2} \boldsymbol{\Gamma}_{\text {prior }}^{-1 / 2} \mathbf{V} \boldsymbol{\Lambda} \mathbf{V}^{T} \boldsymbol{\Gamma}_{\text {prior }}^{-1 / 2} \\
& =\boldsymbol{\Gamma}_{\text {prior }}^{1 / 2} \mathbf{V}(\boldsymbol{\Lambda}-\mathbf{D} \boldsymbol{\Lambda}) \mathbf{V}^{T} \boldsymbol{\Gamma}_{\text {prior }}^{-1 / 2} .
\end{aligned}
$$

Here, it can be seen that $(\boldsymbol{\Lambda}-\mathbf{D} \boldsymbol{\Lambda})=\mathbf{D}$, and therefore, the low-rank approximation of $\mathbf{R}$ is

$$
\mathbf{R} \approx \boldsymbol{\Gamma}_{\text {prior }}^{1 / 2} \mathbf{V}_{k} \mathbf{D}_{k} \mathbf{V}_{k}^{T} \boldsymbol{\Gamma}_{\text {prior }}^{-1 / 2}
$$




\section{References}

Afanasiev, M., C. Boehm, M. van Driel, L. Krischer, M. Rietmann, D. A. May, M. G. Knepley, and A. Fichtner (2019), Modular and flexible spectral-element waveform modelling in two and three dimensions, Geophysical Journal International, 216(3), 1675-1692, doi: 10.1093/gji/ggy469.

Ajo-Franklin, J. B. (2009), Optimal experiment design for time-lapse traveltime tomography, Geophysics, $74(4), \mathrm{Q} 27-\mathrm{Q} 40$.

Aki, K., and W. H. K. Lee (1976), Determination of three-dimensional velocity anomalies under a seismic array using first P arrival times from local earthquakes: 1. A homogeneous initial model, Journal of Geophysical Research (1896-1977), 81(23), 4381-4399, doi: 10. 1029/JB081i023p04381.

Aki, K., and P. G. Richards (2002), Quantitative Seismology, 1-685 pp., University Science Books, Mill Valley, California.

Aki, K., A. Christoffersson, E. Husebye, and C. Powell (1974), Three-dimensional seismic velocity anomalies in the crust and upper-mantle under the USGS, California seismic array, Eos Trans. AGU, 56, 1145.

Alexanderian, A., and A. Saibaba (2018), Efficient D-optimal design of experiments for infinite-dimensional Bayesian linear inverse problems, SIAM Journal on Scientific Computing, 40(5), A2956-A2985, doi: 10.1137/17M115712X.

Alexanderian, A., N. Petra, G. Stadler, and O. Ghattas (2016), A fast and scalable method for A-optimal design of experiments for infinite-dimensional Bayesian nonlinear inverse problems, SIAM J. Sci. Comput., 38(1), A243-A272.

André, M., J. Wiskin, and D. Borup (2013), Clinical results with ultrasound computed tomography of the breast, in Quantitative Ultrasound in Soft Tissues, edited by J. Mamou and M. L. Oelze, pp. 395-432, Springer Netherlands, Dordrecht, doi: 10.1007/978-94-007-6952-6_15.

Athanasiou, A., A. Tardivon, M. Tanter, B. Sigal-Zafrani, J. Bercoff, T. Deffieux, J.-L. Gennisson, M. Fink, and S. Neuenschwander (2010), Breast lesions: Quantitative elas- 


\section{References}

tography with supersonic shear imaging - preliminary results, Radiology, 256(1), 297-303, doi: $10.1148 /$ radiol.10090385.

Atkinson, A. C., and A. N. Donev (1992), Optimum Experimental Designs, 1-344 pp., Oxford University Press, Oxford.

Attia, A., and E. Constantinescu (2018), An Optimal Experimental Design Framework for Adaptive Inflation and Covariance Localization for Ensemble Filters, arXiv e-prints, arXiv:1806.10655.

Attia, A., A. Alexanderian, and A. K. Saibaba (2018), Goal-oriented optimal design of experiments for large-scale Bayesian linear inverse problems, Inverse Problems, 34(9), 095009, doi: 10.1088/1361-6420/aad210.

Auer, L., L. Boschi, T. W. Becker, T. Nissen-Meyer, and D. Giardini (2014), Savani: A variable resolution whole-mantle model of anisotropic shear velocity variations based on multiple data sets, Journal of Geophysical Research: Solid Earth, 119(4), 3006-3034, doi: 10.1002/2013JB010773.

Autier, P., and M. Boniol (2018), Mammography screening: A major issue in medicine, European Journal of Cancer, 90,34-62, doi: https://doi.org/10.1016/j.ejca.2017. 11.002 .

Avron, H., and S. Toledo (2011), Randomized algorithms for estimating the trace of an implicit symmetric positive semi-definite matrix, J. ACM, 58(2), 8:1-8:34.

Barratt, A. (2015), Overdiagnosis in mammography screening: a 45 year journey from shadowy idea to acknowledged reality, BMJ, 350, doi: 10.1136/bmj.h867.

Berg, W. A., J. D. Blume, J. B. Cormack, E. B. Mendelson, D. Lehrer, M. Böhm-Vélez, E. D. Pisano, R. A. Jong, W. P. Evans, M. J. Morton, M. C. Mahoney, L. Hovanessian Larsen, R. G. Barr, D. M. Farria, H. S. Marques, K. Boparai, and f. t. ACRIN 6666 Investigators (2008), Combined Screening With Ultrasound and Mammography vs Mammography Alone in Women at Elevated Risk of Breast Cancer, JAMA, 299(18), 2151-2163, doi: 10.1001/jama.299.18.2151.

Berg, W. A., D. O. Cosgrove, C. J. Doré, F. K. W. Schäfer, W. E. Svensson, R. J. Hooley, R. Ohlinger, E. B. Mendelson, C. Balu-Maestro, M. Locatelli, C. Tourasse, B. C. Cavanaugh, V. Juhan, A. T. Stavros, A. Tardivon, J. Gay, J.-P. Henry, and C. a. Cohen-Bacrie (2012), Shear-wave Elastography Improves the Specificity of Breast US: The BE1 Multinational Study of 939 Masses, Radiology, 262(2), 435-449, doi: 10.1148/radiol.11110640. 
Biller-Andorno, N., and P. Jüni (2014), Abolishing Mammography Screening Programs? A View from the Swiss Medical Board, New England Journal of Medicine, 370(21), 1965-1967, doi: 10.1056/NEJMp1401875, pMID: 24738641.

Boehm, C. (2015), Efficient inversion methods for constrained parameter identification in full-waveform seismic tomography, Ph.D. thesis, Technische Universität München.

Boehm, C., and M. Ulbrich (2015), A semismooth Newton-CG method for constrained parameter identification in seismic tomography, SIAM Journal on Scientific Computing, 37(5), S334-S364, doi: 10.1137/140968331.

Boehm, C., N. Korta Martiartu, N. Vinard, I. J. Balic, and A. Fichtner (2018), Timedomain spectral-element ultrasound waveform tomography using a stochastic quasiNewton method, Proc. SPIE, 10580, 105800H, doi: 10.1117/12.2293299.

Bozdag, E., J. Trampert, and J. Tromp (2011), Misfit functions for full waveform inversion based on instantaneous phase and envelope measurements, Geophysical Journal International, $185(2), 845-870$, doi: 10.1111/j.1365-246X.2011.04970.x.

Bozdag, E., D. Peter, M. Lefebvre, D. Komatitsch, J. Tromp, J. Hill, N. Podhorszki, and D. Pugmire (2016), Global adjoint tomography: first-generation model, Geophysical Journal International, 207(3), 1739-1766, doi: 10.1093/gji/ggw356.

Brem, R. F., L. Tabár, S. W. Duffy, M. F. Inciardi, J. A. Guingrich, B. E. Hashimoto, M. R. Lander, R. L. Lapidus, M. K. Peterson, J. A. Rapelyea, S. Roux, K. J. Schilling, B. A. Shah, J. Torrente, R. T. Wynn, and D. P. Miller (2015), Assessing Improvement in Detection of Breast Cancer with Three-dimensional Automated Breast US in Women with Dense Breast Tissue: The SomoInsight Study, Radiology, 274(3), 663-673, doi: $10.1148 /$ radiol. 14132832

Bunks, C., F. M. Saleck, S. Zaleski, and G. Chavent (1995), Multiscale seismic waveform inversion, Geophysics, 60(5), 1457-1473, doi: 10.1190/1.1443880.

Butler, J. (2002), Gender trouble, Routledge, New York.

Calderón Agudo, O. (2018), Acoustic full-waveform inversion in geophysical and medical imaging, Ph.D. thesis, Imperial College London.

Calderón Agudo, O., L. Guasch, P. Huthwaite, and M. Warner (2018), 3D imaging of the breast using full-waveform inversion, in Proceedings of the International Workshop on Medical Ultrasound Tomography: 1.-3. Nov. 2017, Speyer, Germany, p. 99, KIT Scientific Publishing. 


\section{References}

Camacho, J., L. Medina, J. F. Cruza, J. M. Moreno, and C. Fritsch (2012), Multimodal ultrasonic imaging for breast cancer detection, Archives of Acoustics, 37(3), 253-260.

Carioli, G., M. Malvezzi, T. Rodriguez, P. Bertuccio, E. Negri, and C. L. Vecchia (2017), Trends and predictions to 2020 in breast cancer mortality in Europe, The Breast, 36, 89 - 95, doi: https://doi.org/10.1016/j.breast.2017.06.003.

Carson, P., C. Meyer, A. Scherzinger, and T. Oughton (1981), Breast imaging in coronal planes with simultaneous pulse echo and transmission ultrasound, Science, 214(4525), 1141-1143, doi: 10.1126/science.7302585.

Carson, P. L., and A. Fenster (2009), Anniversary Paper: Evolution of ultrasound physics and the role of medical physicists and the AAPM and its journal in that evolution, Medical Physics, 36(2), 411-428, doi: 10.1118/1.2992048.

Chen, P., L. Zhao, and T. H. Jordan (2007), Full 3D tomography for the crustal structure of the Los Angeles region., Bulletin of the Seismological Society of America, 97, 1094-1120.

Chiolero, A., and N. Rodondi (2014), Lessons From the Swiss Medical Board Recommendation Against Mammography Screening ProgramsSwiss Recommendation Against Screening MammographyViewpoint, JAMA Internal Medicine, 174(10), 1541-1542, doi: 10.1001/jamainternmed.2014.4197.

Chipperfield, A. J., and P. J. Fleming (1995), The MATLAB genetic algorithm toolbox, in IEE Colloquium on Applied Control Techniques Using MATLAB, pp. 10/1-10/4, doi: 10.1049/ic: 19950061.

Claerbout, J. F. (1971), Toward a unified theory of reflector mapping, Geophysics, 36(3), 467-481, doi: 10.1190/1.1440185.

Coles, D., M. Prange, and H. Djikpesse (2015), Optimal survey design for big data, Geophysics, 80(3), P11-P22, doi: 10.1190/geo2014-0425.1.

Crase, E., A. Pica, M. Noble, J. McDonald, and A. Tarantola (1990), Robust elastic nonlinear waveform inversion - Application to real data, Geophysics, 55, 527-538.

Crestel, B., A. Alexanderian, G. Stadler, and O. Ghattas (2017), A-optimal encoding weights for nonlinear inverse problems, with application to the Helmholtz inverse problem, Inverse Problems, 33(7), 074008.

Curtis, A. (1999a), Optimal experiment design: cross-borehole tomographic examples, Geophysical Journal International, 136(3), 637-650.

Curtis, A. (1999b), Optimal design of focused experiments and surveys, Geophysical Journal International, 139(1), 205-215, doi: 10.1046/j.1365-246X.1999.00947.x. 
Curtis, A. (2004a), Theory of model-based geophysical survey and experimental design: Part 1 - Linear problems, The Leading Edge, 23(10), 997-1004.

Curtis, A. (2004b), Theory of model-based geophysical survey and experimental design: Part 2 - Nonlinear problems, The Leading Edge, 23(10), 1112-1117.

Curtis, A., A. Michelini, D. Leslie, and A. Lomax (2004), A deterministic algorithm for experimental design applied to tomographic and microseismic monitoring surveys, Geophysical Journal International, 157(2), 595-606.

Dahlen, F. A., and A. M. Baig (2002), Fréchet kernels for body-wave amplitudes, Geophysical Journal International, 150(2), 440-466, doi: 10.1046/j.1365-246X.2002.01718.x.

Dahlen, F. A., G. Nolet, and S.-H. Hung (2000), Fréchet kernels for finite-frequency traveltimes - I. Theory, Geophysical Journal International, 141(1), 157-174, doi: 10.1046/ j.1365-246X.2000.00070.x.

Dai, W., and G. T. Schuster (2013), Plane-wave least-squares reverse-time migration, Geophysics, 78(4), S165-S177, doi: 10.1190/geo2012-0377.1.

Dapp, R., M. Zapf, and N. V. Ruiter (2011), Geometry-independent speed of sound reconstruction for 3D USCT using apriori information, in 2011 IEEE International Ultrasonics Symposium, pp. 1403-1406, doi: 10.1109/ULTSYM. 2011.0347.

Daston, L. J., and P. Galison (2007), Objectivity, Zone Books.

Davis, A. Y. (2011), Women, race, and class, Vintage.

Deal, M. M., and G. Nolet (1996), Nullspace shuttles, Geophysical Journal International, 124 (2), 372-380, doi: 10.1111/j.1365-246X.1996.tb07027.x.

Derenzo, S. E., T. F. Budinger, R. H. Huesman, J. L. Cahoon, and T. Vuletich (1981), Imaging properties of a positron tomograph with 280 BGO Crystals, IEEE Transactions on Nuclear Science, 28(1), 81-89, doi: 10.1109/TNS.1981.4331144.

Dette, H., and T. E. O'Brien (1999), Optimality criteria for regression models based on predicted variance, Biometrika, 86(1), 93-106.

Douma, H., D. Yingst, I. Vasconcelos, and J. Tromp (2010), On the connection between artifact filtering in reverse-time migration and adjoint tomography, Geophysics, 75(6), S219-S223, doi: 10.1190/1.3505124.

Duric, N., and P. Littrup (2018), Breast ultrasound tomography, in Breast Imaging, edited by C. M. Kuzmiak, chap. 6, IntechOpen, Rijeka, doi: 10.5772/intechopen.69794. 
Duric, N., P. Littrup, A. Babkin, D. Chambers, S. Azevedo, A. Kalinin, R. Pevzner, M. Tokarev, E. Holsapple, O. Rama, and R. Duncan (2005), Development of ultrasound tomography for breast imaging: Technical assessment, Medical Physics, 32(5), 1375-1386, doi: 10.1118/1.1897463.

Duric, N., P. Littrup, L. Poulo, A. Babkin, R. Pevzner, E. Holsapple, O. Rama, and C. Glide (2007), Detection of breast cancer with ultrasound tomography: First results with the Computed Ultrasound Risk Evaluation (CURE) prototype, Medical Physics, 34(2), 773785, doi: 10.1118/1.2432161.

Duric, N., P. Littrup, S. Schmidt, C. Li, O. Roy, L. Bey-Knight, R. Janer, D. Kunz, X. Chen, J. Goll, A. Wallen, F. Zafar, V. Allada, E. West, I. Jovanovic, K. Li, and W. Greenway (2013), Breast imaging with the SoftVue imaging system: first results, Proc. SPIE, 8675, doi: $10.1117 / 12.2002513$.

Duric, N., P. Littrup, C. Li, O. Roy, S. Schmidt, X. Cheng, J. Seamans, A. Wallen, and L. Bey-Knight (2014), Breast imaging with SoftVue: initial clinical evaluation, Proc. SPIE, 9040, 90400V, doi: 10.1117/12.2043768.

Duric, N., P. L. M.D., C. Li, O. Roy, S. Schmidt, J. Seamans, A. Wallen, and L. Bey-Knight (2015), Whole breast tissue characterization with ultrasound tomography, Proc. SPIE, 9419, doi: $10.1117 / 12.2083203$.

Dziewonski, A. M. (1984), Mapping the lower mantle: Determination of lateral heterogeneity in P velocity up to degree and order 6, Journal of Geophysical Research: Solid Earth, 89 (B7), 5929-5952, doi: 10.1029/JB089iB07p05929.

Dziewonski, A. M., and J. H. Woodhouse (1987), Global Images of the Earth's Interior, Science, 236(4797), 37-48, doi: 10.1126/science.236.4797.37.

Ellsworth, W. L., and R. Y. Koyanagi (1977), Three-dimensional crust and mantle structure of Kilauea Volcano, Hawaii, Journal of Geophysical Research (1896-1977), 82(33), 53795394, doi: 10.1029/JB082i033p05379.

Ermert, L., K. Sager, M. Afanasiev, C. Boehm, and A. Fichtner (2017), Ambient Seismic Source Inversion in a Heterogeneous Earth: Theory and Application to the Earth's Hum, Journal of Geophysical Research: Solid Earth, 122(11), 9184-9207, doi: 10.1002/ 2017 JB014738.

Ferlay, J., M. Colombet, I. Soerjomataram, T. Dyba, G. Randi, M. Bettio, A. Gavin, O. Visser, and F. Bray (2018), Cancer incidence and mortality patterns in Europe: Estimates for 40 countries and 25 major cancers in 2018, European Journal of Cancer, 103, 356 - 387, doi: https://doi.org/10.1016/j.ejca.2018.07.005. 
Feyerabend, P. K. (1962), Explanation, reduction and empiricism, in Crítica: Revista Hispanoamericana de Filosofía, edited by H. Feigl and G. Maxwell, pp. 103-106.

Fichtner, A. (2010), Full Seismic Waveform Modelling and Inversion, 1-343 pp., Springer, Heidelberg.

Fichtner, A., and J. Trampert (2011a), Hessian kernels of seismic data functionals based upon adjoint techniques, Geophysical Journal International, 185(2), 775-798.

Fichtner, A., and J. Trampert (2011b), Resolution analysis in full waveform inversion, Geophysical Journal International, 187(3), 1604-1624, doi: 10.1111/j.1365-246X . 2011. 05218.x.

Fichtner, A., and T. van Leeuwen (2015), Resolution analysis by random probing, Journal of Geophysical Research: Solid Earth, 120(8), 5549-5573, doi: 10.1002/2015JB012106.

Fichtner, A., H.-P. Bunge, and H. Igel (2006), The adjoint method in seismology - I. Theory, Physics of the Earth and Planetary Interiors, 157, 86-104.

Fichtner, A., B. L. N. Kennett, H. Igel, and H. P. Bunge (2008), Theoretical background for continental- and global-scale full-waveform inversion in the time-frequency domain, Geophysical Journal International, 175(2), 665-685, doi: 10.1111/j.1365-246X.2008. 03923.x.

Fichtner, A., D.-P. van Herwaarden, M. Afanasiev, S. Simutè, L. Krischer, Y. Çubuk Sabuncu, T. Taymaz, L. Colli, E. Saygin, A. Villaseñor, J. Trampert, P. Cupillard, H.P. Bunge, and H. Igel (2018), The Collaborative Seismic Earth Model: Generation 1, Geophysical Research Letters, 45(9), 4007-4016, doi: 10.1029/2018GL077338.

Flath, H., L. Wilcox, V. Akçelik, J. Hill, B. van Bloemen Waanders, and O. Ghattas (2011), Fast algorithms for Bayesian uncertainty quantification in large-scale linear inverse problems based on low-rank partial hessian approximations, SIAM Journal on Scientific Computing, 33(1), 407-432, doi: 10.1137/090780717.

Friederich, W. (2003), The S-velocity structure of the East Asian mantle from inversion of shear and surface waveforms, Geophysical Journal International, 153, 88-102.

Gallagher, K., and M. Sambridge (1994), Genetic algorithms: A powerful tool for large-scale nonlinear optimization problems, Computers and Geosciences, 20(7), 1229 - 1236.

Gauthier, O., J. Virieux, and A. Tarantola (1986), Two-dimensional nonlinear inversion of seismic waveforms: Numerical results, Geophysics, 51(7), 1387-1403, doi: 10.1190/1. 1442188 . 


\section{References}

Gemmeke, H., and N. Ruiter (2007), 3D ultrasound computer tomography for medical imaging, Nuclear Instruments and Methods in Physics Research Section A: Accelerators, Spectrometers, Detectors and Associated Equipment, 580(2), 1057 - 1065, doi: https: //doi.org/10.1016/j.nima.2007.06.116.

Gemmeke, H., R. Dapp, T. Hopp, M. Zapf, and N. V. Ruiter (2014), An improved 3D Ultrasound Computer Tomography system, in 2014 IEEE International Ultrasonics Symposium, pp. 1009-1012, doi: 10.1109/ULTSYM.2014.0247.

Gemmeke, H., T. Hopp, M. Zapf, C. Kaiser, and N. V. Ruiter (2017), 3D ultrasound computer tomography: Hardware setup, reconstruction methods and first clinical results, Nuclear Instruments and Methods in Physics Research Section A: Accelerators, Spectrometers, Detectors and Associated Equipment, 873, 59 - 65, doi: https: //doi.org/10.1016/j.nima.2017.07.019, imaging 2016.

Glover, G. (1977), Computerized time-of-flight ultrasonic tomography for breast examination, Ultrasound in Medicine $\mathcal{E}$ Biology, 3(2), 117 - 127, doi: https://doi.org/10. 1016/0301-5629(77) 90064-3.

Glover, G. H., and J. C. Sharp (1977), Reconstruction of ultrasound propagation speed distributions in soft tissue: Time-of-flight tomography, IEEE Transactions on Sonics and Ultrasonics, 24(4), 229-234, doi: 10.1109/T-SU.1977.30936.

Goncharsky, A., S. Y. Romanov, and S. Y. Seryozhnikov (2016), A computer simulation study of soft tissue characterization using low-frequancy ultrasonic tomography, Ultrasonics, 67, 136-150.

Green, P. S., L. F. Schaefer, E. D. Jones, and J. R. Suarez (1974), A New, HighPerformance Ultrasonic Camera, pp. 493-503, Springer US, Boston, MA, doi: 10.1007/ 978-1-4757-0827-1_27.

Greenleaf, J., and S. Johnson (1975), Algebraic reconstruction of spatial distribution of acoustic speed and attenuation in tissues from time-of-flight and amplitude profiles, in Natl Bur Stand Spec Publ, 453 ed., pp. 109-119.

Greenleaf, J. F., and R. C. Bahn (1981), Clinical imaging with transmissive ultrasonic computerized tomography, IEEE Transactions on Biomedical Engineering, BME-28(2), 177-185, doi: 10.1109/TBME.1981.324789.

Greenleaf, J. F., S. A. Johnson, and R. C. Bahn (1977), Quantitative cross-sectional imaging of ultrasound parameters, in 1977 Ultrasonics Symposium, pp. 989-995, doi: 10.1109/ULTSYM.1977.196985. 
Guest, T., and A. Curtis (2009), Iteratively constructive sequential design of experiments and surveys with nonlinear parameter-data relationships, Journal of Geophysical Research: Solid Earth, 114(B4), b04307.

Haber, E., L. Horesh, and L. Tenorio (2008), Numerical methods for experimental design of large-scale linear ill-posed inverse problems, Inverse Problems, 24 (5), 055012.

Hadamard, J. (1952), Lectures on Cauchy's problem in linear partial differential equations, Dover, New York, NY.

Hager, W. (1989), Updating the inverse of a matrix, SIAM Review, 31(2), 221-239, doi: $10.1137 / 1031049$.

Haraway, D. (1991), Simians, Cyborgs, and Women, Routledge, New York, doi: https: //doi.org/10.4324/9780203873106.

Harding, S. (1992), Rethinking standpoint epistemology: What is "strong objectivity?", The Centennial Review, 36(3), 437-470.

Hardt, M., and F. Scherbaum (1994), The design of optimum networks for aftershock recordings, Geophysical Journal International, 117(3), 716-726.

Harvey, J. A., and V. E. Bovbjerg (2004), Quantitative assessment of mammographic breast density: Relationship with breast cancer risk, Radiology, 230(1), 29-41, doi: 10. 1148/radiol.2301020870, pMID: 14617762.

Heyser, R., and D. Croissette (1974), A new ultrasonic imaging system using time delay spectrometry, Ultrasound in Medicine and Biology, 1(2), 119 -131, doi: https://doi. org/10.1016/0301-5629(74)90002-7.

Hosseini, K. (2016), Global multiple-frequency seismic tomography using teleseismic and core-diffracted body waves.

Hosseini, K., K. J. Matthews, K. Sigloch, G. E. Shephard, M. Domeier, and M. Tsekhmistrenko (2018), SubMachine: Web-Based Tools for Exploring Seismic Tomography and Other Models of Earth's Deep Interior, Geochemistry, Geophysics, Geosystems, 19(5), 1464-1483, doi: 10.1029/2018GC007431.

Hounsfield, G. N. (1973), Computerized transverse axial scanning (tomography): Part 1. Description of system, The British Journal of Radiology, 46(552), 1016-1022, doi: 10.1259/0007-1285-46-552-1016, pMID: 4757352.

Hubbard, R. (1990), The politics of women's biology, Rutgers University Press. 


\section{References}

Hung, S.-H., F. Dahlen, and G. Nolet (2000), Fréchet kernels for finite-frequency traveltimes - II. Examples, Geophysical Journal International, 141(1), 175-203, doi: 10.1046/j . 1365-246X.2000.00072.x.

Hung, S.-H., Y. Shen, and L.-Y. Chiao (2004), Imaging seismic velocity structure beneath the Iceland hot spot: A finite frequency approach, Journal of Geophysical Research: Solid Earth, 109(B8), doi: 10.1029/2003JB002889.

Hutchinson, M. (1990), A stochastic estimator of the trace of the influence matrix for Laplacian smoothing splines, Communications in Statistics - Simulation and Computation, $19(2), 433-450$.

Ihrig, A., and G. Schmitz (2018), Accelerating nonlinear speed of sound reconstructions using a randomized block Kaczmarz algorithm, in 2018 IEEE International Ultrasonics Symposium (IUS), pp. 1-9, doi: 10.1109/ULTSYM.2018.8580199.

Iyer, H. M., and K. Hirahara (1993), Seiemic tomography, Theory and Practice, Chapman $\&$ Hall.

Jensen, J. A., S. I. Nikolov, K. L. Gammelmark, and M. H. Pedersen (2006), Synthetic aperture ultrasound imaging, Ultrasonics, 44, e5-e15, doi: https://doi.org/10.1016/ j.ultras.2006.07.017, proceedings of Ultrasonics International (UI'05) and World Congress on Ultrasonics (WCU).

Jensen, T. L., J. H. Jørgensen, P. C. Hansen, and S. H. Jensen (2012), Implementation of an optimal first-order method for strongly convex total variation regularization, BIT Numerical Mathematics, 52(2), 329-356, doi: 10.1007/s10543-011-0359-8.

Johnson, S., T. Abbott, R. Bell, M. Berggren, D. Borup, D. Robinson, J. Wiskin, S. Olsen, and B. Hanover (2007), Non-invasive breast tissue characterization using ultrasound speed and attenuation, in Acoustical Imaging, edited by M. P. André, pp. 147-154, Springer Netherlands, Dordrecht.

Kak, A. C., and K. A. Dines (1978), Signal processing of broadband pulsed ultrasound: Measurement of attenuation of soft biological tissues, IEEE Transactions on Biomedical Engineering, BME-25(4), 321-344, doi: 10.1109/TBME.1978.326259.

Kaplan, S. S. (2014), Automated Whole Breast Ultrasound, Radiologic Clinics of North America, 52(3), 539 - 546, doi: https://doi.org/10.1016/j.rcl.2014.01.002.

Keller, E. F. (1991), Language and ideology in evolutionary theory: Reading cultural norms into natural law, in The boundaries of humanity: Humans, animals, machines, edited by E. F. Keller and H. E. Longino, pp. 85-102, University of California Press Berkeley. 
Keller, E. F. (1996), Reflections on Gender and Science, Yale University Press.

Kennett, B. L. N., J. Stipčević, and A. Gorbatov (2015), Spiral-Arm Seismic Arrays, Bulletin of the Seismological Society of America, 105(4), 2109-2116, doi: 10.1785/ 0120140354.

Khodja, M. R., M. D. Prange, and H. A. Djikpesse (2010), Guided Bayesian optimal experimental design, Inverse Problems, 26 (5), 055008.

Klawiter, M. (2004), Breast cancer in two regimes: the impact of social movements on illness experience, Sociology of Health \& Illness, 26(6), 845-874, doi: 10.1111/j.1467-9566. 2004.421_1.x.

Klawiter, M. (2008), The Biopolitics of Breast Cancer: Changing Cultures of Disease and Activism, Minneapolis: University of Minnesota Press.

Korta Martiartu, N., C. Boehm, N. Vinard, I. Jovanović Balic, and A. Fichtner (2017), Optimal experimental design to position transducers in ultrasound breast imaging, Proc. SPIE, 10139, 101390M, doi: 10.1117/12.2252514.

Korta Martiartu, N., C. Boehm, V. Hapla, H. Maurer, I. J. Balic, and A. Fichtner (2019), Optimal experimental design for joint reflection-transmission ultrasound breast imaging: From ray- to wave-based methods, The Journal of the Acoustical Society of America, 146 (2), 1252-1264, doi: 10.1121/1.5122291.

Korta Martiartu, N., C. Boehm, and A. Fichtner (2020), 3D Wave-Equation-Based FiniteFrequency Tomography for Ultrasound Computed Tomography, IEEE Transactions on Ultrasonics, Ferroelectrics, and Frequency Control, pp. 1-1, doi: 10.1109/TUFFC. 2020. 2972327.

Latour, B. (1999), Pandora's hope: essays on the reality of science studies, Harvard university press.

Lavarello, R., F. Kamalabadi, and W. D. O'Brien (2006), A regularized inverse approach to ultrasonic pulse-echo imaging, IEEE Transactions on Medical Imaging, 25(6), 712-722, doi: $10.1109 /$ TMI . 2006.873297.

Lavarello, R. J., and A. J. Hesford (2013), Methods for forward and inverse scattering in ultrasound tomography, in Quantitative Ultrasound in Soft Tissues, edited by J. Mamou and M. L. Oelze, pp. 345-394, Springer Netherlands, Dordrecht, doi: 10.1007/978-94-007-6952-6_14.

Lavarello, R. J., and M. L. Oelze (2009), Tomographic reconstruction of three-dimensional volumes using the distorted born iterative method, IEEE Transactions on Medical Imaging, 28(10), 1643-1653, doi: 10.1109/TMI.2009.2026274. 


\section{References}

Lenox, M. W., J. Wiskin, M. A. Lewis, S. Darrouzet, D. Borup, and S. Hsieh (2015), Imaging performance of Quantitative Transmission Ultrasound, International Journal of Biomedical Imaging, 2015(454028), doi: 10.1155/2015/454028.

Lerner, B. H. (2002), Breast cancer activism: past lessons, future directions, Nature Reviews Cancer, 2(3), 225-230, doi: 10.1038/nrc744.

Li, C., N. Duric, and L. Huang (2008), Comparison of ultrasound attenuation tomography methods for breast imaging, Proc. SPIE, 6920, 692015, doi: 10.1117/12.771433.

Li, C., N. Duric, P. Littrup, and L. Huang (2009), In vivo breast sound-speed imaging with ultrasound tomography, Ultrasound in Medicine and Biology, 35(10), 1615 - 1628.

Li, C., G. S. Sandhu, O. Roy, N. Duric, V. Allada, and S. Schmidt (2014), Toward a practical ultrasound waveform tomography algorithm for improving breast imaging, Proc. SPIE, 9040, doi: $10.1117 / 12.2043686$.

Li, S., M. Jackowski, D. P. Dione, T. Varslot, L. H. Staib, and K. Mueller (2010), Refraction corrected transmission ultrasound computed tomography for application in breast imaging, Medical Physics, 37(5), 2233-2246, doi: 10.1118/1.3360180.

Liu, C., C. Xue, B. Zhang, G. Zhang, and C. He (2018), The application of an ultrasound tomography algorithm in a novel ring 3D ultrasound imaging system, Sensors, 18(5), doi: $10.3390 / \mathrm{s} 18051332$.

Liu, Q., and Y. Gu (2012), Seismic imaging: From classical to adjoint tomography, Tectonophysics, 566-567, 31 -66, doi: https://doi.org/10.1016/j.tecto.2012.07.006.

Lou, Y., W. Zhou, T. P. Matthews, C. M. Appleton, and M. A. Anastasio (2017), Generation of anatomically realistic numerical phantoms for photoacoustic and ultrasonic breast imaging, Journal of Biomedical Optics, 22 (4), 41015, doi: 10.1117/1. JBO .22.4.041015.

Luo, Y., and G. T. Schuster (1991), Wave-equation traveltime inversion, Geophysics, 56 (5), 645-653, doi: 10.1190/1.1443081.

Malik, B., R. Terry, J. Wiskin, and M. Lenox (2018), Quantitative transmission ultrasound tomography: Imaging and performance characteristics, Medical Physics, 45 (7), 3063-3075, doi: $10.1002 / \mathrm{mp} .12957$.

Maranò, S., D. Fäh, and Y. M. Lu (2014), Sensor placement for the analysis of seismic surface waves: sources of error, design criterion and array design algorithms, Geophysical Journal International, 197(3), 1566-1581, doi: 10.1093/gji/ggt489. 
Marquering, H., F. Dahlen, and G. Nolet (1999), Three-dimensional sensitivity kernels for finite-frequency traveltimes: the banana-doughnut paradox, Geophysical Journal International, 137(3), 805-815, doi: 10.1046/j.1365-246x.1999.00837.x.

Martin, E. (1991), The egg and the sperm: How science has constructed a romance based on stereotypical male-female roles, Signs: Journal of Women in Culture and Society, $16(3)$, 485-501, doi: $10.1086 / 494680$.

Martin, J., L. C. Wilcox, C. Burstedde, and O. Ghattas (2012), A Stochastic Newton MCMC Method for Large-Scale Statistical Inverse Problems with Application to Seismic Inversion, SIAM J. Scientific Computing, 34.

Mast, T. D. (2000), Empirical relationships between acoustic parameters in human soft tissues, Acoustics Research Letters Online, 1(2), 37-42, doi: 10.1121/1.1336896.

Matthews, T. P., K. Wang, C. Li, N. Duric, and M. A. Anastasio (2017), Regularized dual averaging image reconstruction for full-wave ultrasound computed tomography, IEEE Transactions on Ultrasonics, Ferroelectrics, and Frequency Control, 64(5), 811-825.

Maurer, H., S. Greenhalgh, and S. Latzel (2009), Frequency and spatial sampling strategies for crosshole seismic waveform spectral inversion experiments, Geophysics, 74 (6), WCC79WCC89, doi: 10.1190/1.3157252.

Maurer, H., A. Curtis, and D. E. Boerner (2010), Recent advances in optimized survey design, Geophysics, 75(5), 75A177-75A194.

Maurer, H., A. Nuber, N. Korta Martiartu, F. Reiser, C. Boehm, E. Manukyan, C. Schmelzbach, and A. Fichtner (2017), Optimized experimental design in the context of seismic full waveform inversion and seismic waveform imaging, in Advances in Geophysics, vol. 58, edited by L. Nielsen, pp. 1 - 45, Elsevier, doi: https://doi.org/10. 1016/bs .agph.2017.10.001.

Mercerat, E. D., and G. Nolet (2012), On the linearity of cross-correlation delay times in finite-frequency tomography, Geophysical Journal International, 192(2), 681-687, doi: 10.1093/gji/ggs017.

Mitchell, B. J., C. C. Cheng, and W. Stauder (1977), A three-dimensional velocity model of the lithosphere beneath the new Madrid seismic zone, Bulletin of the Seismological Society of America, 67(4), 1061-1074.

Montelli, R., G. Nolet, F. A. Dahlen, G. Masters, E. R. Engdahl, and S.-H. Hung (2004), Finite-frequency tomography reveals a variety of plumes in the mantle, Science, 303(5656), 338-343, doi: 10.1126/science.1092485. 


\section{References}

Moore, S. G., P. J. Shenoy, L. Fanucchi, J. W. Tumeh, and C. R. Flowers (2009), Costeffectiveness of MRI compared to mammography for breast cancer screening in a high risk population, BMC Health Services Research, 9(1), 9, doi: 10.1186/1472-6963-9-9.

Mora, P. (1989), Inversion = migration + tomography, Geophysics, 54(12), 1575-1586, doi: $10.1190 / 1.1442625$.

Nakata, N., L. Gualtieri, and A. Fichtner (2019), Seismic Ambient Noise, Cambridge University Press.

Nishida, K., and Y. Fukao (2007), Source distribution of Earth's background free oscillations, Journal of Geophysical Research: Solid Earth, 112(B6), doi: 10.1029/ 2006JB004720, b06306.

Nolet, G. (1985), Solving or resolving inadequate and noisy tomographic systems, Journal of Computational Physics, 61(3), 463 - 482, doi: https://doi.org/10.1016/ 0021-9991 (85) 90075-0.

Nolet, G. (2008), A Breviary of Seismic Tomography: Imaging the Interior of the Earth and Sun, Cambridge University Press, doi: 10.1017/CB09780511984709.

Osuch, J. R., K. Silk, C. Price, J. Barlow, K. Miller, A. Hernick, and A. Fonfa (2012), A historical perspective on breast cancer activism in the United States: From education and support to partnership in scientific research, Journal of Women's Health, 21(3), 355-362, doi: 10.1089/jwh.2011.2862, pMID: 22132763.

Ozmen, N., R. Dapp, M. Zapf, H. Gemmeke, N. V. Ruiter, and K. W. A. van Dongen (2015), Comparing different ultrasound imaging methods for breast cancer detection, IEEE Transactions on Ultrasonics, Ferroelectrics, and Frequency Control, 62(4), 637-646, doi: 10.1109/TUFFC. 2014.006707.

Paige, C. C., and M. A. Saunders (1982a), LSQR: An Algorithm for Sparse Linear Equations and Sparse Least Squares, ACM Trans. Math. Softw., 8(1), 43-71, doi: $10.1145 / 355984.355989$.

Paige, C. C., and M. A. Saunders (1982b), Lsqr: An algorithm for sparse linear equations and sparse least squares, ACM Trans. Math. Softw., 8(1), 43-71, doi: 10.1145/355984. 355989.

Penrose, R. (1955), A generalized inverse for matrices, Mathematical Proceedings of the Cambridge Philosophical Society, 51(3), 406-413, doi: 10.1017/S0305004100030401.

Pérez-Liva, M., J. L. Herraiz, L. Medina-Valdés, J. Camacho, C. Fritsch, B. E. Treeby, and J. M. Udías (2015), Regularization of image reconstruction in ultrasound computed 
tomography, in 2015 IEEE Nuclear Science Symposium and Medical Imaging Conference (NSS/MIC), pp. 1-3, doi: 10.1109/NSSMIC. 2015.7582217.

Pérez-Liva, M., J. L. Herraiz, J. M. Udías, E. Miller, B. T. Cox, and B. E. Treeby (2017a), Time domain reconstruction of sound speed and attenuation in ultrasound computed tomography using full wave inversion, The Journal of the Acoustical Society of America, 141(3), 1595-1604, doi: 10.1121/1.4976688.

Pérez-Liva, M., J. L. Herraiz, J. M. Udías, E. Miller, B. T. Cox, and B. E. Treeby (2017b), Time domain reconstruction of sound speed and attenuation in ultrasound computed tomography using full wave inversion, The Journal of the Acoustical Society of America, 141(3), 1595-1604, doi: 10.1121/1.4976688.

Perry, N., M. Broeders, C. de Wolf, S. Törnberg, R. Holland, and L. von Karsa (2008), European guidelines for quality assurance in breast cancer screening and diagnosis. Fourth edition - summary document, Annals of Oncology, 19 (4), 614-622, doi: 10.1093/ annonc/mdm 481 .

Peterlik, I., R. Jirik, N. Ruiter, and J. Jan (2008), Regularized image reconstruction for ultrasound attenuation transmission tomography, Radioengineering, 17(2), 125-132.

Plessix, R.-E. (2006), A review of the adjoint-state method for computing the gradient of a functional with geophysical applications, Geophysical Journal International, 167, 495-503.

Potter, E. (2001), Gender and Boyle's Law of Gases, Indiana University Press.

Pratt, R. G. (1999), Seismic waveform inversion in the frequency domain; Part 1, Theory and verification in a physical scale model, Geophysics, 64(3), 888-901, doi: 10.1190/1. 1444597.

Pratt, R. G. (2018), Medical ultrasound tomography: lessons from exploration geophysics, in Proceedings of the International Workshop on Medical Ultrasound Tomography: 1.-3. Nov. 2017, Speyer, Germany, p. 65, KIT Scientific Publishing.

Pratt, R. G., L. Huang, N. Duric, and P. Littrup (2007), Sound-speed and attenuation imaging of breast tissue using waveform tomography of transmission ultrasound data, Proc. SPIE, 6510, 65104S.

Rabinowitz, N., and D. M. Steinberg (1990), Optimal configuration of a seismographic network: A statistical approach, Bulletin of the Seismological Society of America, 80(1), $187-196$. 


\section{References}

Radon, J. (1917), Uber die Bestimmung von Funktionen durch ihre Integralwerte langs gewisser Mannigfaltigkeiten. Berichte Schsische Akademie der Wissenschaften, Mathematisch-Physikalische Klasse, 69, 262-277.

Rawlinson, N., S. Pozgay, and S. Fishwick (2010), Seismic tomography: A window into deep Earth, Physics of the Earth and Planetary Interiors, $178(3), 101$ - 135, doi: https: //doi.org/10.1016/j.pepi.2009.10.002.

Romanowicz, B. A. (1979), Seismic structure of the upper mantle beneath the United States by three-dimensional inversion of body wave arrival times, Geophysical Journal of the Royal Astronomical Society, 57(2), 479-506, doi: 10.1111/j.1365-246X.1979. tb04790.x.

Romanowicz, B. A. (1980), A study of large-scale lateral variations of $\mathrm{P}$ velocity in the upper mantle beneath western Europe, Geophysical Journal International, 63(1), 217232, doi: $10.1111 / \mathrm{j} .1365-246 \mathrm{X} .1980 . \mathrm{tb02617}$.x.

Roux, E., and X. Garcia (2014), Optimizing an experimental design for a CSEM experiment: methodology and synthetic tests, Geophysical Journal International, 197, 135-148.

Roy, O., I. Jovanović, A. Hormati, R. Parhizkar, and M. Vetterli (2010), Sound speed estimation using wave-based ultrasound tomography: theory and GPU implementation, Proc. SPIE, 7629, 76290J, doi: 10.1117/12.844691.

Roy, O., S. Schmidt, C. Li, V. Allada, E. West, D. Kunz, and N. Duric (2013), Breast imaging using ultrasound tomography: From clinical requirements to system design, in 2013 IEEE International Ultrasonics Symposium (IUS), pp. 1174-1177, doi: 10.1109/ ULTSYM. 2013.0300.

Roy, O., M. A. H. Zuberi, R. G. Pratt, and N. Duric (2016), Ultrasound breast imaging using frequency domain reverse time migration, Proc. SPIE, 9790, 97900B, doi: 10.1117/ 12.2218366 .

Ruiter, N. V., M. Zapf, T. Hopp, R. Dapp, and H. Gemmeke (2012), Phantom image results of an optimized full 3D USCT, Proc. SPIE, 8320, 832005, doi: 10.1117/12.911245.

Ruiter, N. V., M. Zapf, T. Hopp, H. Gemmeke, and K. W. A. van Dongen (2017), USCT data challenge, Proc. SPIE, 10139, 101391N, doi: 10.1117/12.2272593.

Ruiter, N. V., M. Zapf, T. Hopp, H. Gemmeke, K. W. A. van Dongen, J. Camacho, J. L. Herraiz, M. P. Liva, and J. M. Udías (2018), USCT reference data base: conclusions from the first SPIE USCT data challenge and future directions, Proc. SPIE, 10580, 105800Q, doi: 10.1117/12.2293063. 
Sager, K., L. Ermert, C. Boehm, and A. Fichtner (2017), Towards full waveform ambient noise inversion, Geophysical Journal International, 212(1), 566-590, doi: 10.1093/gji/ $\operatorname{ggx} 429$.

Sak, M., N. Duric, P. Littrup, L. Bey-Knight, H. Ali, P. Vallieres, M. E. Sherman, and G. L. Gierach (2017), Using speed of sound imaging to characterize breast density, Ultrasound in Medicine and Biology, 43(1), 91 - 103, doi: https://doi.org/10.1016/ j.ultrasmedbio.2016.08.021.

Sandhu, G. Y., C. Li, O. Roy, S. Schmidt, and N. Duric (2015), Frequency domain ultrasound waveform tomography: breast imaging using a ring transducer, Physics in Medicine \& Biology, 60(14), 5381.

Sandhu, G. Y., E. West, C. Li, O. Roy, and N. Duric (2017), 3D frequency-domain ultrasound waveform tomography breast imaging, Proc. SPIE, 10139, 1013909, doi: $10.1117 / 12.2254399$.

Sardanelli, F., G. M. Giuseppetti, P. Panizza, M. Bazzocchi, A. Fausto, G. Simonetti, V. Lattanzio, and A. Del Maschio (2004), Sensitivity of MRI versus mammography for detecting foci of multifocal, multicentric breast cancer in fatty and dense breasts using the whole-breast pathologic examination as a gold standard, American Journal of Roentgenology, 183(4), 1149-1157.

Saslow, D., C. Boetes, W. Burke, S. Harms, M. O. Leach, C. D. Lehman, E. Morris, E. Pisano, M. Schnall, S. Sener, R. A. Smith, E. Warner, M. Yaffe, K. S. Andrews, C. A. Russell, and for the American Cancer Society Breast Cancer Advisory Group (2007), American cancer society guidelines for breast screening with MRI as an adjunct to mammography, CA: A Cancer Journal for Clinicians, 57(2), 75-89, doi: 10.3322/canjclin.57.2.75.

Schiebinger, L. (2000), Has feminism changed science?, Signs, 25(4), 1171-1175.

Schmidt, S., N. Duric, C. Li, O. Roy, and Z.-F. Huang (2011), Modification of Kirchhoff migration with variable sound speed and attenuation for acoustic imaging of media and application to tomographic imaging of the breast, Medical Physics, 38(2), 998-1007, doi: $10.1118 / 1.3539552$.

Schomberg, H. (1978), An improved approach to reconstructive ultrasound tomography, Journal of Physics D: Applied Physics, 11(15), L181.

Schwarzenberg, G. F., M. Zapf, and N. V. Ruiter (2007), P3D-5 aperture optimization for 3D ultrasound computer tomography, in 2007 IEEE Ultrasonics Symposium Proceedings, pp. 1820-1823, doi: 10.1109/ULTSYM.2007.458. 


\section{References}

Shen, X. (2010), Near-surface velocity estimation by weighted early-arrival waveform inversion, pp. 1975-1979, doi: 10.1190/1.3513230.

Sigloch, K., and G. Nolet (2006), Measuring finite-frequency body-wave amplitudes and traveltimes, Geophysical Journal International, 167(1), 271-287, doi: 10.1111/j . 1365-246X.2006.03116.x.

Sigloch, K., N. McQuarrie, and G. Nolet (2008), Two-stage subduction history under North America inferred from multiple-frequency tomography, Nature Geoscience, 1, doi:10.1038/ngeo231.

Simmons, N. A., A. M. Forte, L. Boschi, and S. P. Grand (2010), GyPSuM: A joint tomographic model of mantle density and seismic wave speeds, Journal of Geophysical Research: Solid Earth, 115(B12), doi: 10.1029/2010JB007631.

Simmons, N. A., S. C. Myers, G. Johannesson, E. Matzel, and S. P. Grand (2015), Evidence for long-lived subduction of an ancient tectonic plate beneath the southern Indian Ocean, Geophysical Research Letters, 42(21), 9270-9278, doi: 10.1002/2015GL066237.

Simonetti, F., L. Huang, and N. Duric (2007), On the spatial sampling of wave fields with circular ring apertures, Journal of Applied Physics, 101(8), 083103, doi: 10.1063/ 1.2717086 .

Slamon, D., W. Godolphin, L. Jones, J. Holt, S. Wong, D. Keith, W. Levin, S. Stuart, J. Udove, A. Ullrich, and a. et (1989), Studies of the her-2/neu proto-oncogene in human breast and ovarian cancer, Science, 244 (4905), 707-712, doi: 10.1126/science. 2470152.

Spetzler, J., J. Trampert, and R. Snieder (2001), Are we exceeding the limits of the Great Circle Approximation in global surface wave tomography?, Geophysical Research Letters, 28(12), 2341-2344, doi: 10.1029/2000GL012691.

Stavros, A. T., D. Thickman, C. L. Rapp, M. A. Dennis, S. H. Parker, and G. A. Sisney (1995), Solid breast nodules: use of sonography to distinguish between benign and malignant lesions., Radiology, 196(1), 123-134, doi: 10.1148/radiology.196.1.7784555.

Stotzka, R., N. V. Ruiter, T. O. Mueller, R. Liu, and H. Gemmeke (2005), High resolution image reconstruction in ultrasound computer tomography using deconvolution, Proc. SPIE, 5750, 315-326, doi: 10.1117/12.595149.

Stutzmann, E., F. Ardhuin, M. Schimmel, A. Mangeney, and G. Patau (2012), Modelling long-term seismic noise in various environments, Geophysical Journal International, 191 (2), 707-722, doi: 10.1111/j.1365-246X.2012.05638.x. 
Tape, C., Q. Liu, A. Maggi, and J. Tromp (2010), Seismic tomography of the southern California crust based on spectral-element and adjoint methods, Geophysical Journal International, 180(1), 433-462, doi: 10.1111/j.1365-246X.2009.04429.x.

Tarantola, A. (1984), Linearized inversion of seismic reflection data, Geophysical Prospecting, 32(6), 998-1015, doi: 10.1111/j.1365-2478.1984.tb00751.x.

Tarantola, A. (1988a), Theoretical background for the inversion of seismic waveforms including elasticity and attenuation, pure and applied geophysics, 128(1), 365-399, doi: 10.1007/BF01772605.

Tarantola, A. (1988b), Theoretical background for the inversion of seismic waveforms, including elasticity and attenuation, Pure and Applied Geophysics, 128, 365-399.

Tarantola, A. (2005), Inverse Problem Theory and Methods for Model Parameter Estimation, 1-339 pp., Society for Industrial and Applied Mathematics, Philadelphia, doi: $10.1137 / 1.9780898717921$.

Taskin, U., J. van der Neut, and K. W. A. van Dongen (2018), Redatuming for breast ultrasound, in 2018 IEEE International Ultrasonics Symposium (IUS), pp. 1-9, doi: 10.1109/ULTSYM. 2018.8580049 .

Tian, Y., K. Sigloch, and G. Nolet (2009), Multiple-frequency SH-wave tomography for the western US upper mantle, Geophysical Journal International, 178, 1384-1402.

Tikhonov, A. (1963), Solution of incorrectly formulated problems and the regularization method, Soviet Math. Dokl., 5, 1035/1038.

Tong, J., F. A. Dahlen, G. Nolet, and H. Marquering (1998a), Diffraction effects upon finite-frequency travel times: A simple 2-D example, Geophysical Research Letters, 25, $1983-1986$.

Tong, J., F. A. Dahlen, G. Nolet, and H. Marquering (1998b), Diffraction effects upon finite-frequency travel times: A simple 2-D example, Geophysical Research Letters, 25(11), 1983-1986, doi: 10.1029/98GL01291.

Tromp, J., C. Tape, and Q. Liu (2005), Seismic tomography, adjoint methods, time reversal and banana-doughnut kernels, Geophysical Journal International, 160(1), 195-216, doi: $10.1111 / j .1365-246 X .2004 .02453 . x$.

Tuana, N. (1989), Feminism and Science, Indiana University Press.

van Herwaarden, D. P., C. Boehm, M. Afanasiev, S. Thrastarson, L. Krischer, J. Trampert, and A. Fichtner (2019), Accelerated full-waveform inversion using dynamic mini-batches, preprint. 


\section{References}

van Leeuwen, T., and F. J. Herrmann (2013), Fast waveform inversion without sourceencoding, Geophysical Prospecting, 61(s1), 10-19.

Vinard, N., N. Korta Martiartu, C. Boehm, I. Jovanović Balic, and A. Fichtner (2018), Optimized transducer configuration for ultrasound waveform tomography in breast cancer detection, Proc. SPIE, 10580, 105800I, doi: 10.1117/12.2293600.

Virieux, J., and S. Operto (2009), An overview of full-waveform inversion in exploration geophysics, Geophysics, 74(6), WCC1-WCC26, doi: 10.1190/1.3238367.

Wang, K., T. Matthews, F. Anis, C. Li, N. Duric, and M. A. Anastasio (2015), Waveform inversion with source encoding for breast sound speed reconstruction in ultrasound computed tomography, IEEE Transactions on Ultrasonics, Ferroelectrics, and Frequency Control, 62 (3), 475-493, doi: 10.1109/TUFFC.2014.006788.

Warner, M., and L. Guasch (2016), Adaptive waveform inversion: Theory, Geophysics, 81(6), R429-R445, doi: 10.1190/geo2015-0387.1.

Williamson, P. R. (1991), A guide to the limits of resolution imposed by scattering in ray tomography, Geophysics, 56(2), 202-207, doi: 10.1190/1.1443032.

Wiskin, J., D. T. Borup, S. A. Johnson, and M. Berggren (2012), Non-linear inverse scattering: High resolution quantitative breast tissue tomography, The Journal of the Acoustical Society of America, 131(5), 3802-3813, doi: 10.1121/1.3699240.

Wiskin, J., D. Borup, S. Johnson, M. Andre, J. Greenleaf, Y. Parisky, and J. Klock (2013), Three-dimensional nonlinear inverse scattering: Quantitative transmission algorithms, refraction corrected reflection, scanner design and clinical results, Proceedings of Meetings on Acoustics, 19(1), doi: 10.1121/1.4800267.

Wiskin, J. W., D. T. Borup, E. Iuanow, J. Klock, and M. W. Lenox (2017), 3-D nonlinear acoustic inverse scattering: Algorithm and quantitative results, IEEE Transactions on Ultrasonics, Ferroelectrics, and Frequency Control, 64 (8), 1161-1174, doi: 10.1109/TUFFC. 2017.2706189 .

Woodward, M. J. (1986), Wave equation tomography, Ph.D. thesis, Stanford University, Stanford, CA, USA.

Woodward, M. J. (1992), Wave-equation tomography, Geophysics, 57(1), 15-26, doi: 10. 1190/1.1443179.

Yomogida, K. (1992), Fresnel zone inversion for lateral heterogeneities in the Earth, Pure and Applied Geophysics, 138, 391-406. 
Yoshizawa, K., and G. Ekström (2010), Automated multimode phase speed measurements for high-resolution regional-scale tomography: application to North America, Geophysical Journal International, 183, 1538-1558.

Yoshizawa, K., and B. L. N. Kennett (2004), Multi-mode surface wave tomography for the Australian region using a 3-stage approach incorporating finite-frequency effects, Journal of Geophysical Research: Solid Earth, 109, doi:10.1029/2002JB002254.

Zhou, Y., F. A. Dahlen, G. Nolet, and G. Laske (2005), Finite-frequency effects in global surface-wave tomography, Geophysical Journal International, 163(3), 1087-1111, doi: $10.1111 / \mathrm{j} .1365-246 \mathrm{X} .2005 .02780 . \mathrm{x}$.

Zhou, Y., G. Nolet, F. A. Dahlen, and G. Laske (2006a), Global upper-mantle structure from finite-frequency surface-wave tomography, Journal of Geophysical Research: Solid Earth, 111 (B4), doi: 10.1029/2005JB003677.

Zhou, Y., G. Nolet, F. A. Dahlen, and G. Laske (2006b), Global upper-mantle structure from finite-frequency surface-wave tomography, Journal of Geophysical Research: Solid Earth, 111, doi:10.1029/2005JB003677.

Zografos, G., D. Koulocheri, P. Liakou, M. Sofras, S. Hadjiagapis, M. Orme, and V. Marmarelis (2013), Novel technology of multimodal ultrasound tomography detects breast lesions, European Radiology, 23(3), 673-683, doi: 10.1007/s00330-012-2659-z. 



\section{Acknowledgments/Eskerrak/Agradecimientos}

Anything that I could acknowledge in these lines would not be fair with the truth. There are stories that one simply does not share, but definitely shape the process and outcome of a thesis, including us. This is a long-term work, in which one experiences many different emotional stages and not all are necessarily nice to live. Let us admit it. This is a tough period. Its end is just liberating. This is why I will like to acknowledge those who have empowered me in the process and helped me to conclude it.

During this thesis I have learned a lot from Christian. I am deeply grateful to your supervision and advices, my work has always substantially improved with your feedback. I would also like to thank Andreas for the trust and for giving me the opportunity to re-start the thesis in a topic that fulfills my personal interests more; also for hosting and supervising me in Utrecht. This was very empowering for me. During that time, Josep became my mentor. I always felt that he believed in my potential, and this had a direct impact on my own perception about it. Moltes gràcies de veritat. Thanks also to Nicole and Hartmut for hosting me in Karlsruhe. You have a beautiful team there, and it has been a pleasure to feel part of it during my internship. This thesis has also benefited from the suggestions and discussions with Ivana, Hansruedi and Vaclav, who became co-authors of the chapters presented here.

Of course, my colleagues from the SWP group and the institute have been essential in this process. They offered me affection, caring, friendship, and acceptance. My most genuine thanks to them, especially to Korbinian, Maria, Fang, Neda, Saule, Marie, Steffi, Yavor, Alejandro, Pili, Moritz. Thanks for being there. Also to Sabine, Sigrid, Monika and Elisabeth.

En este largo proceso he encontrado compañerxs que me han dado su amor, cariño y fuerza. Definitivamente, este amor ha atravesado todos los aspectos de mi vida, incluida esta tesis. Mi periodo en Barcelona fue maravilloso junto a Savitri, Agnese, Xisco, Pablo, Rafa, Jordi, Nassim, Ruth. Zürich, en cambio, embelleció gracias a las compañeras de F.I.S.T., hermosas luchadoras y soñadoras de incansable energía. Hauen artien, Nekane eta Urhaitz, zeuen energixek ilusioz bete deuz geure bixizek. Erreferente bat zarie.

Neure eskerrik zintzoenak ama, aita eta Gaizkari, baite neure hurbileneko lagunei, Siv, Maria, Deiene, Leyre, Josu. Zuok argi utzi dostazue neure poztasune eta osasune dala garrantzitsuena, beste edozeren ganetik, eta oso babestute sentidu naz une oro, neure erabaki guztixetan. Asko maite zaitzuet, eta urrun egon arren hamen egon zarie nigaz, 


\section{Acknowledgments}

egunero neure bixotzeko lekutxu baten. Tesixe prozesu luze bat izen da nire kasuen, behin Bartzelonan hasi ta oin Zurichen bukatzen dotena. Azken tarte honetan hurbil egon ez garen arren, Miren ta Oskar, zuen laguntzie ezinbestekoa izen da, askotan ganezka ein dosten prozesu honetan indertsu irauteko. Eskerrik asko alkarregaz ikesi eta sortu dogunagatik.

Mis amores porteños no podrían faltar en estos agradecimientos. Ivan, nos descubrimos en la Karcelona, en aquel primer intento de tesis que abandoné con alegría. Ansiaba reinventarme y en esa busqueda te encontré. Desde entonces me has enseñado a saber quién es la persona que quiero ser. Juntxs nos hemos cuestionado todo, nos hemos amargado la existencia, reinventado nuestra identidad, luchado con rabia, transformado los deseos, mariconeado con orgullo y soñado en Valpo. Si ahora he podido terminar la tesis, ha sido gracias a la fuerza de ese desaprendizaje. Mil gracias también a Gerardo, Sofi, Silvia, Pablo, lxs cabrxs de la UNAB y UTFSM, lxs quiltrxs, por ser parte de aquellos momentos tan hermosos que he rescatado de mi memoria cada vez que he necesitado en Zurich.

Finally, I would like to thank Laura. The last two years have been very beautifully intense next to you. I could feel your constant love and support that made me definitely happier and stronger to confront this process. Your understanding, trust, and patience have been essential to keep my motivation alive. Asko gutzut, laztana. Eskerrik asko. 


\section{Naiara Korta Martiartu}

\section{Education}

Since 2016 PhD studies, Seismology and Wave Physics group, ETH Zurich, Switzerland. Topic: Knowledge transfer from seismology to imaging breast tissue with ultrasound

2009-2010 MSc in Geophysics, University of Barcelona and Ramon Llull University, Barcelona, Catalonia, Spain.

Thesis: Comparative analysis of misfit functionals for time-domain full waveform inversion

2007-2009 Undergraduate studies in Mathematics (4 semesters), University of Basque Country, Leioa, Basque Country, Spain.

2004-2009 Bachelor in Physics, University of Basque Country, Leioa, Basque Country, Spain.

\section{Working and teaching experience}

06/2017; Teaching assistant, ETH Zurich, Switzerland.

06/2018; Geophysikalisches Feldpraktikum: Geoelectrics

$06 / 2019$

2015 University lecturer in classical mechanics.

- Universidad Andrés Bello (UNAB), Viña del Mar, Chile.

- Pontificia Universidad Católica de Valparaiso (PUCV), Valparaiso, Chile.

- Universidad Técnica Federico Santa María (UTFSM), Valparaiso, Chile.

Teaching method: Active learning in Physics.

2010-2014 Research assistant, Institute of Marine Sciences (Spanish National Research Council, CSIC), Barcelona, Catalonia, Spain.

Topic: Time-domain multi-parameter 2D full waveform inversion for acoustic-elastic scenarios in seismic exploration

\section{Geophysical field work}

10/2011- Geophysicist, Institute of Marine Sciences (Spanish National Research Council, 11/2011 CSIC), Barcelona.

Multi-Channel Seismic Survey GEOMARGEN and TOPOMED Cruises in the Alboran Sea, Strait of Gibraltar and Gulf of Cadiz on board the Spanish research vessel 'Sarmiento de Gamboa'.

04/2010 - Geophysicist, Institute of Marine Sciences (Spanish National Research Council,

05/2010 CSIC), Barcelona.

Multi-Channel Seismic Survey MEDOC Cruise in the Tyrrhenian Sea on board the Spanish research vessel 'Sarmiento de Gamboa'. 


\section{International experience}

05/2019- Research internship, Karlsruhe Institute of Technology, Germany.

06/2019 Application of finite-frequency reconstruction algorithms to lab data acquired from full 3D acquisition systems. Host: PD. Dr. Nicole Ruiter

01/2012- Research internship, Utrecht University, Netherlands.

04/2012 Development of full waveform inversion code and new optimization techniques based on the Hessian approximation. Host: Dr. Andreas Fichtner

\section{Courses attended}

2018 Critiques of scientific objectivity, ETH Zurich, Switzerland. Lecturer: Prof. Roy Wagner

2017 Inverse Problem Theory - A Bayesian Approach, ETH Zurich, Switzerland. Lecturer: Prof. Klaus Mosegaard

2016 Wave Propagation, ETH Zurich, Switzerland. Lecturer: Prof. Donat Fäh

2016 College in Medical Physics, ICTP Trieste, Italy.

2015 Active learning Methods in Basic Sciences (16h), Universidad Técnica Federico Santa María (UTFSM), Valparaiso, Chile.

\section{Scholarships}

2010 - 2014 JAE-PreDoc scholarship of the Spanish National Research Council (CSIC). Invited talks

- Seismology group, Department of Earth Science, University of Oxford, Nov. 2018.

○ Symposium "Imaging the Earth and Beyond", Utrecht University, June 2018.

- Geophysical colloquium, ETH Zurich, June 2017. 


\section{Permissions}


This Agreement between Ms. Naiara Korta Martiartu ("You") and The American Association for the Advancement of Science ("The American Association for the Advancement of Science") consists of your license details and the terms and conditions provided by The American Association for the Advancement of Science and Copyright Clearance Center.

License Number

License date

Licensed Content Publisher

Licensed Content Publication

Licensed Content Title

Licensed Content Author

Licensed Content Date

Licensed Content Volume

Licensed Content Issue

Volume number

Issue number

Type of Use

Requestor type

Format

Portion

Number of figures/tables

Order reference number

Title of your thesis / dissertation

Expected completion date

Estimated size(pages)

Requestor Location

Total
4631911118493

Jul 18, 2019

The American Association for the Advancement of Science

Science

Breast imaging in coronal planes with simultaneous pulse echo and transmission ultrasound

PL Carson,CR Meyer,AL Scherzinger,TV Oughton

Dec 4, 1981

214

4525

214

4525

Thesis / Dissertation

Scientist/individual at a research institution

Print and electronic

Figure

1

Tomography across scales: Knowledge transfer from seismology to imaging breast tissue with ultrasound

Oct 2019

150

Ms. Naiara Korta Martiartu Sonneggstrasse 5

Zurcih, Zurich 8092

Switzerland

Attn: Ms. Naiara Korta Martiartu

$0.00 \mathrm{CHF}$

Terms and Conditions

American Association for the Advancement of Science TERMS AND CONDITIONS

Regarding your request, we are pleased to grant you non-exclusive, non-transferable permission, to republish the AAAS material identified above in your work identified above, subject to the terms and conditions herein. We must be contacted for permission for any uses other than those specifically identified in your request above.

The following credit line must be printed along with the AAAS material: "From [Full Reference Citation]. Reprinted with permission from AAAS." 
May 20, 2020

This Agreement between Ms. Naiara Korta Martiartu ("You") and Elsevier ("Elsevier") consists of your license details and the terms and conditions provided by Elsevier and Copyright Clearance Center.

License Number $\quad 4833240964042$

License date

May 20, 2020

Licensed Content

Publisher

Elsevier

Nuclear Instruments and Methods in Physics

Licensed C
Publication

Research Section A: Accelerators,

Spectrometers, Detectors and Associated

Equipment

3D ultrasound computer tomography: Hardware

Licensed Content Title setup, reconstruction methods and first clinical results

Licensed Content Hartmut Gemmeke,Torsten Hopp,Michael

Author

Zapf,Clemens Kaiser,Nicole V. Ruiter

Licensed Content Date Nov 21, 2017

Licensed Content

Volume

873

Licensed Content

Issue

$\mathrm{n} / \mathrm{a}$

Licensed Content

Pages 
End Page

Type of Use

Portion

Number of

figures/tables

/illustrations

Format

Are you the author of this Elsevier article?

Will you be

translating?

Title

Institution name

Expected presentation date

Portions

Figure 1

Ms. Naiara Korta Martiartu

Sonneggstrasse 5

Requestor Location

Zurcih, Zurich 8092

Switzerland

Attn: Ms. Naiara Korta Martiartu

Publisher Tax ID

GB 494627212

Total

0.00 EUR

Terms and Conditions 